\title{
DISCLAIMER
}

This report was prepared as an account of work sponsored by an agency of the United States Government. Neither the United States Government nor any agency thereof, nor any of their employees, makes any warranty, express or implied, or assumes any legal liability or responsibility for the accuracy, completeness, or usefulness of any information, apparatus, product, or process disclosed, or represents that its use would not infringe privately owned rights. Reference herein to any specific commercial product, process, or service by trade name, trademark, manufacturer, or otherwise does not necessarily constitute or imply its endorsement, recommendation, or favoring by the United States Government or any agency thereof. The views and opinions of authors expressed herein do not necessarily state or reflect those of the United States Government or any agency thereof.

\section{GEOTHERMAL DIRECT USE DEVELOPMENTS \\ IN THE UNITED STATES}

August 1988

by

Paul J. Lienau

Gene Culver

John w. Lund

Geo-Heat center

oregon Institute of Technology

Klamath Falls, OR 97601

Prepared for the

U.S. Department of Energy

Idaho operation office

Under contract No. DE-FG07-87ID 12693 


\section{DISCLAIMER}

This report was prepared as an account of work sponsored by an agency of the United States Government. Neither the United States Government nor any agency Thereof, nor any of their employees, makes any warranty, express or implied, or assumes any legal liability or responsibility for the accuracy, completeness, or usefulness of any information, apparatus, product, or process disclosed, or represents that its use would not infringe privately owned rights. Reference herein to any specific commercial product, process, or service by trade name, trademark, manufacturer, or otherwise does not necessarily constitute or imply its endorsement, recommendation, or favoring by the United States Government or any agency thereof. The views and opinions of authors expressed herein do not necessarily state or reflect those of the United States Government or any agency thereof. 


\section{DISCLAIMER}

Portions of this document may be illegible in electronic image products. Images are produced from the best available original document. 


\section{DISCLAIMER}

This report was prepared as an account of work sponsored by the United States Government. Neither the United States no the United States Department of Energy, nor any of their employees, nor any of their contractors, subcontractors, or their empoloyees, makes any warranty, expressed or implied, or assumes any legal responsibility for the accuracy, completeness or usefulness of any information, apparatus, product or process disclosed, or represents that its use would not infringe privately-owned rights. 


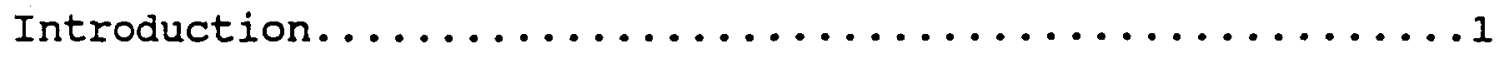

State of Direct Use Development..................

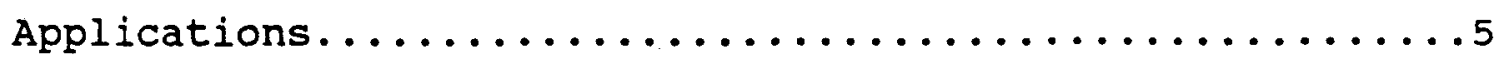

Industrial...........................

Heat Pumps............................

Resorts and Pools.......................

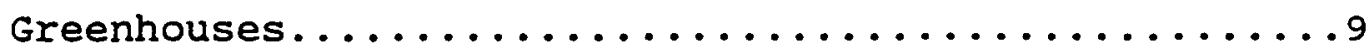

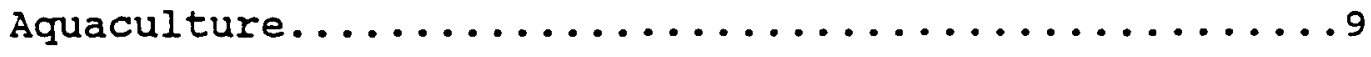

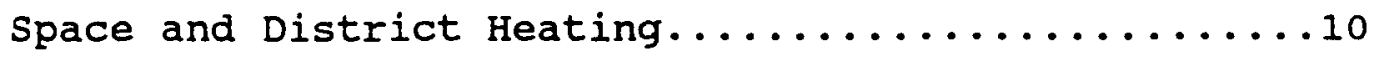

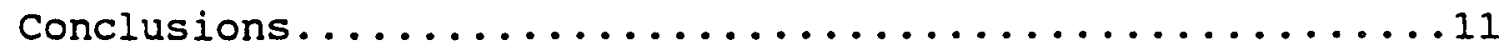

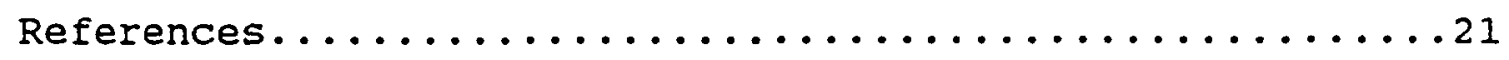

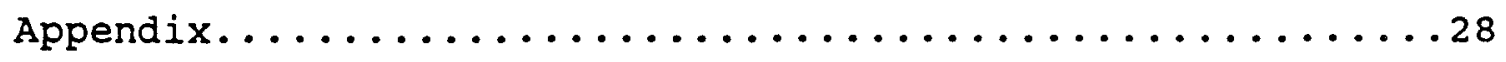


GEOTHERMAL DIRECT USE DEVELOPMENTS IN THE UNITED STATES

\section{ABSTRACT}

Direct heat use of geothermal energy in the United states is recognized as one of the alternative energy resources that has proven itself technically and economically, and is commercially available. Developments include space conditioning of buildings, district heating, groundwater heat pumps, greenhouse heating, industrial processing, aquaculture, and swimming pool heating. Forty-four states have experienced significant geothermal direct use development in the last ten years. The total installed capacity is 5.7 billion Btu/hr $\left(1,700 \mathrm{MW}_{t}\right)$, with an annual energy use of nearly 17,000 billion Btu/Yr $(4.5$ million barrels of oil energy equivalent). In this report we provide an overview of how and where geothermal energy is used, the extent of that use, the economics and growth trends. The data is based on an extensive site data gathering effort by the Geo-Heat center in the spring of 1988, under contract to the U.S. Department of Energy.

\section{INTRODUCTION}

Expansion of geothermal energy projects will broaden the energy base of our country in the near term, further confirm an alternate energy technology based on domestic energy supplies, and thus could contribute in the long term to our nation's energy security.

Studies of the U.S. Geological survey state that the resource base for geothermal is very large. Table 1 provides a summary of thermal energy available from low-to-moderate temperature identified geothermal systems in the United States (Muffler,1978 and Reed, 1982) and Figure 1 shows the location of known and potential geothermal resources.

Low-temperature geothermal resources occur in two types of geothermal systems-hydrothermal convection and conduction dominated which are quantified in Table 1. In hydrothermal convection systems, upward circulation of water transports thermal energy to reservoirs at shallow depths or to the surface. These systems commonly occur in regions of active tectonism and above-normal heat flow, such as much of the Western United states. In conduction-dominated systems, there exists high vertical temperature gradients in rocks that include aquifers of significant lateral extent. These conditions occur beneath many deep sedimentary basins throughout the United States (Reed, 1982). 
Table 1. Summary of Thermal Energy from Low-to-Moderate Temperature Identified Geothermal Systems in the U.S.

\begin{tabular}{|c|c|c|c|}
\hline System & $\begin{array}{l}\text { Resource } \\
\text { Temp. } \\
\left({ }^{\circ} \mathrm{c}\right)\end{array}$ & No. Systems & $\begin{array}{c}\text { Resource } \\
\text { (We11head Energy) } \\
\left(\times 10^{15} \text { Btu }\right)\end{array}$ \\
\hline $\begin{array}{l}\text { Hydrothermal- } \\
\text { Convection }\end{array}$ & $90^{<90}$ to 150 & $\begin{array}{r}1,119 \\
163\end{array}$ & $\begin{array}{r}29.4 \\
167.0\end{array}$ \\
\hline $\begin{array}{l}\text { Conduction- } \\
\text { Dominated to } 3 \mathrm{~km}\end{array}$ & $<90$ & $\frac{42}{1,324}$ & $\frac{53.1}{249.5}$ \\
\hline
\end{tabular}

a. Assumes a recovery factor of 0.25

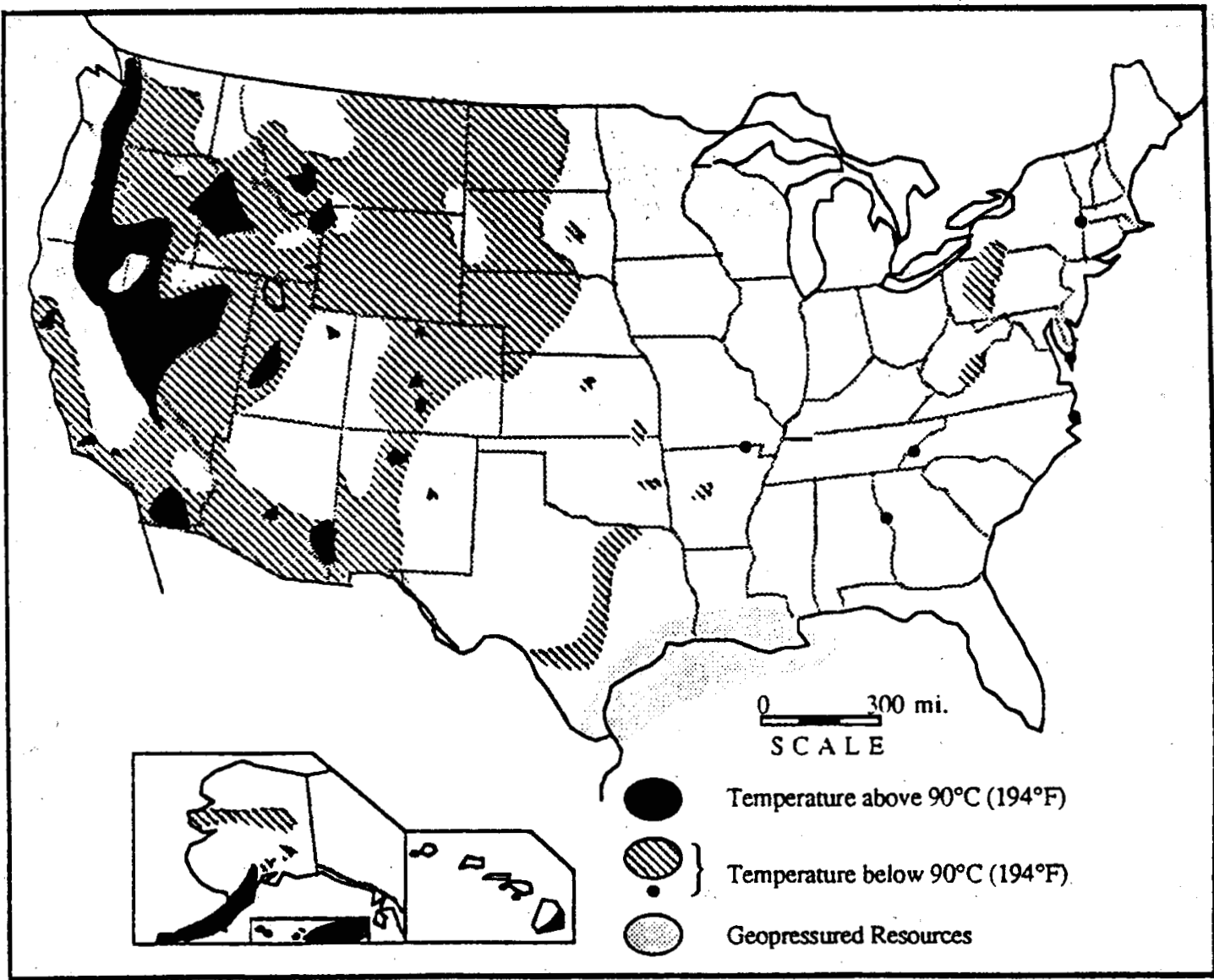

Figure 1. Known and potential geothermal resources (UURI). 
Most people think of geothermal energy as a western states resource; however, there are significant projects developing this resource for space conditioning and district heating where low (45 to $70^{\circ} \mathrm{F}$ ) temperature ground water aquifers exist in central and eastern states. Groundwater and earth coupled (vertical configuration) heat pump systems depend upon the average groundwater temperature. The temperature of the ground and aquifers below 100 feet is controlled by the geothermal gradient and thus are considered geothermal. Figure 2 shows the range of aquifer temperatures and the heating load factor which is the ratio of the average annual heat load to the peak heat load for one year.

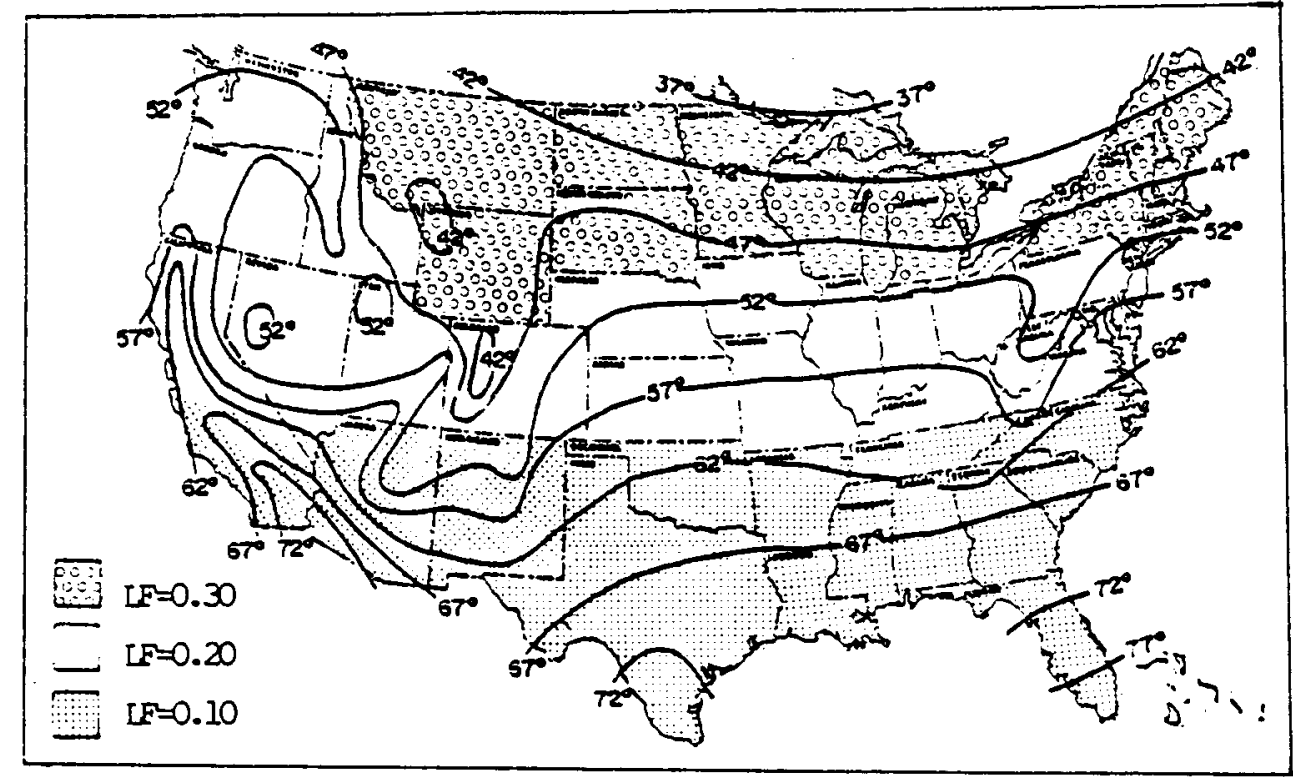

Figure 2. Groundwater temperature in wells ranging from 50 to 150 ft in depth and heating load factor map (National Water Well Institute).

Historically, direct uses of geothermal energy in the United states were by small resorts and limited space and district heating systems. The oil price shocks of the 1970's revived interest in the use of geothermal resources as an alternative energy source. Beginning in 1978, the U.S. Department of Energy initiated numerous programs that caused significant growth in this industry. These programs involved technical assistance to developers, the preparation of project feasibility studies for potential users, cost sharing of demonstration projects (space and district heating, industrial, aqricultural and aquaculture), resource assessments, loan guarantees, support of state resource and commercialization activities, and others. Also adding to the growth were various federal and state tax credit programs (Lunis, 1988). 
This study identified 67,170 sites using geothermal energy for industrial, heat pumps, resorts and pools, aquaculture, greenhouses, space and district heating. Appendix A provides a description for over 370 locations and references for the source of information. The most significant findings resulting from the recent inventory of direct use projects are, the large number of installed ground water heat pumps, primarily a phenomena of the 1980 's, and the increasing use of geothermal energy for aquaculture applications. The installed capacity of swimming pools and spas using geothermal fluid is much larger than previously reported. Several district heating systems are under development, others are expanding and the potential is very large for over 300 cities located near geothermal resources. These factors are incorporated into the comparison of direct use projects' summaries of previous investigations (Lunis, 1988) and the current inventory (Table 2). Other conditions contributing to the large increase of recorded geothermal direct use projects are the intensive effort in identifying heretofore unknown projects, and the increasing industry growth rate.

Table 2. Comparison of Inventories of Installed Geothermal Direct Use Projects

\begin{tabular}{|c|c|c|c|c|}
\hline \multirow[b]{2}{*}{ Application } & \multicolumn{2}{|c|}{ Prior survey $^{a}$} & \multicolumn{2}{|c|}{ Current survey } \\
\hline & $\begin{array}{l}\text { Quantity } \\
\text { (each) }\end{array}$ & $\begin{array}{cl}\text { Annual } & \text { Energy } \\
(x 10 & \text { Btu } / y)\end{array}$ & $\begin{array}{l}\text { Quantity } \\
\text { (each) }\end{array}$ & $\begin{aligned} \text { Annual } & \text { Energy } \\
\left(\times 10^{9}\right. & \mathrm{Btu} / \mathrm{y})\end{aligned}$ \\
\hline Industrial & 7 & 9625 & 16 & 8625 \\
\hline Heat Pumps & -- & -- & 66,135 & 3602 \\
\hline Pools/spás & 85 & 240 & 114 & 1452 \\
\hline Aquaculture & 8 & 400 & 18 & 970 \\
\hline Greenhouses & 37 & 385 & 35 & 852 \\
\hline Space Heating & 655 & 485 & 829 & 744 \\
\hline District Heating & 20 & 405 & 23 & 700 \\
\hline 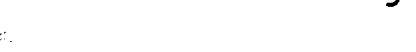 & 812 & 11,540 & 67,170 & 16,945 \\
\hline
\end{tabular}

a. B. C. Lunis, Idaho National Engineering Laboratory, presentation made to the American Association of Petroleum Geologists, Rocky Mountain Section Meeting, september 15, 1987. The data base is restricted to earlier (1980) surveys that had limited updating.

The inventory identified 44 states (Figure 3) that have substantial geothermal energy developments representing an estimated annual energy utilization of nearly 17,000 billion Btu/yr. Tables 3 and 4 summarize by state these developments. 


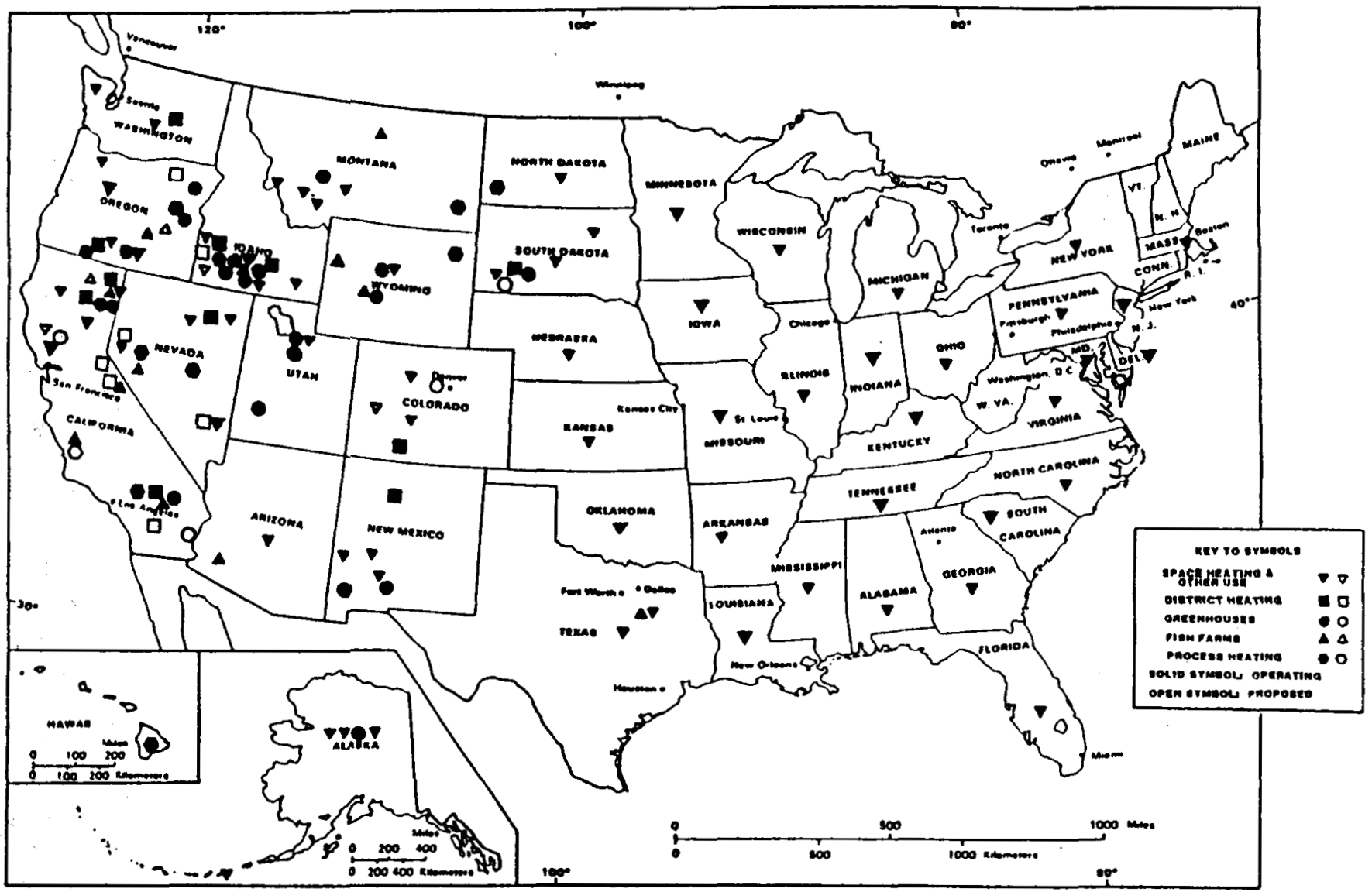

Figure 3. Location of direct heat projects in the United states.

The state with the largest use is Wyoming, the next largest states are Montana and California, due to secondary oil recovery $\left(8,200 \times 10^{9}\right.$ Btu/yr) from the oil fields North and South Dakota, Wyoming and Montana. However, fluctuating oil prices may impact the degree to which it is utilized.

\section{APPLICATIONS}

Figure 3 shows the distribution of use according to application, which excludes the secondary oil recovery operation. Below each application is described, explaining how the resource is used, economics and growth trends.

Industrial

Industrial applications mostly need the higher temperatures while space heating and agriculture predominantly use low temperatures. Examples of industrial uses include; enhanced oil recovery $\left(200^{\circ} \mathrm{F}\right)$; heap leaching operations to extract precious metals $(230 \mathrm{~F})$; dehydration of vegetables $(270 \mathrm{~F})$; mushroom growing $\left(235^{\circ} \mathrm{F}\right) \dot{\delta}$ and others worldwige such as pulp and paper processing $\left(400^{\circ} \mathrm{F}\right)$; hay drying $\left(363^{\circ} \mathrm{F}\right)$; timber drying $\left(200^{\circ} \mathrm{F}\right)$; and diatomaceous earth drying $\left(360^{\circ} \mathrm{F}\right)$. 


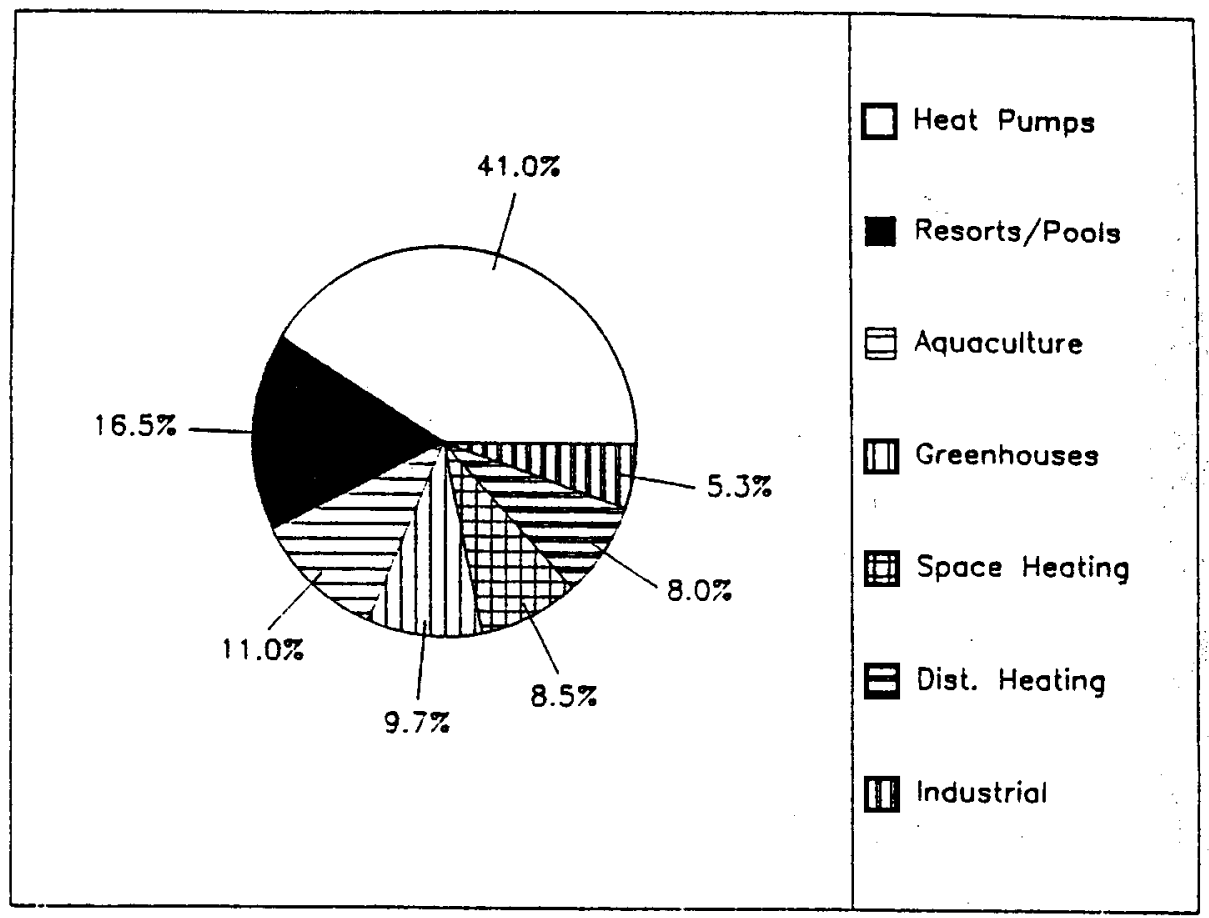

Figure 4. Geothermal use by application in the U. S. (excludes industrial enhanced oil recovery, 8,156 billion Btu/yr).

Drying and dehydration may be the two most important process uses of geothermal energy. A variety of vegetable and fruit products can be considered for dehydration at geothermal temperatures. Dehydration processes involve either continuous belt conveyors or batch dryers, using low temperature air from 100 to $200^{\circ} \mathrm{F}$. Blowers and exhaust fans move the air over coils through which the geothermal fluid flows. The heated air then flows through the beds of vegetables or fruits on conveyors, to evaporate the moisture. Geothermal Food Processors near Fernly Nevada dehydrate onions, garlic, celery, and carrots using $270^{\circ} \mathrm{F}$ geothermal fluid. This saves an estimated $86.0 \mathrm{billion} \mathrm{Btu} / \mathrm{Yr}$ which is equivalent to replacing $119 \times 10^{6}$ cubic feet of natural gas corresponding to a savings of about $\$ 350,000$ per year. This plant has been operating since 1978 .

When oil is produced, only about a third of the oil in the ground can be recovered by simply pumping production wells. Secondary recovery, the injection of water to move oil toward production wells, is often used to recover up to an additional third of the original oil. In the oil fields of North and South Dakota, Wyoming and Montana, geothermal fluid is produced with the oil from several deep zones. This fluid is often between 140 and $212^{\circ} \mathrm{F}$ as it is produced at the surface, and this heat is extremely useful in the secondary recovery of additional oil. Efficient secondary oil recovery is a function of the temperature and chemical compatibility of the injected water compared to the oil formation. To calculate the benefit from the use of geothermal 
fluid, a comparison is made between the geothermal water and the energy needed to heat surface water to the same temperature. The estimated begefit for secondary oil recovery in the four states is $8,156.2 \times 10^{9} \mathrm{Btu} / \mathrm{Yr}$, however fluctuating oil prices may impact the degree to which it is utilized.

Large volumes of geothermal fluid are separated from oil production or are produced from other zones for use in secondary oil recovery. The injected water use in the four states mentioned above comes primarily from the Dakota sandstone and the deeper Madison Limestone. Dakota aquifer water ranges between 140 and $170^{\circ} \mathrm{F}$ and Madison aquifer water ranges between 160 and $212^{\circ} \mathrm{F}$.

New developments in 1988, are the use of geothermal fluids for enhanced heap leaching of precious metals in Nevada by heating the cyanide circuit. This represents and additional 300 billion Btulyr of energy use or a 3 percent growth.

\section{Heat Pumps}

Ground water or earth coupled heat pumps are systems designed to use the earth as a heat source and/or sink. Geothermal fluid is either pumped or water is circulated through a pipe buried vertically in the ground, transfering thermal energy to or from a water-to-refrigerant heat exchanger in the heat pump. In a typical reversible heat pump the heat exchanger serves as the condenser or the evaporator, depending upon whether the heat pump is in a cooling or a heating mode. These types of heat pumps offer several distinct advantages over the use of air as a source or sink; the ground is usually at a more favorable temperature than the air, and the liquid-refrigerant exchanger permits a closer temperature approach than an air-refrigerant exchanger. The total effect is that the ground water and earth coupled systems shows improved performance when compared to air source systems.

operating costs for a ground water heat pump will vary widely, depending on the size and construction of the building, the heating and cooling load, the geothermal fluid temperature and the price of electricity. Here is an example of a $10 \mathrm{~g}, 000$ square foot office building with a heating load of $2.0 \times 10^{6} \mathrm{Btu} / \mathrm{hr}$ and a cooling load of 250 tons for an equivalent 2,000 full load hrs per year. The temperature of the geothermal fluid is $75^{\circ} \mathrm{F}$, pumped from a $200 \mathrm{ft}$ level with a discharge pressure of 40 psi. A comparison of the estimated annual operating costs are:

1. Electric furnace

$\begin{array}{lll}\text { Heating: } & 879,000 \mathrm{kWh} \text { electricity \& } \$ .05 / \mathrm{kWh} & \$ 43,950 \\ \text { Cooling: } & 420,500 \mathrm{kWh} \text { electricity @ } \$ .05 / \mathrm{kWh} & 21,025 \\ & & \$ 61,975\end{array}$


2. Natural Gas

$\begin{array}{lll}\text { Heating: } & 37,500 \text { Therms \& } \$ .50 / \text { Therm } & 18,750 \\ \text { Cooling: } & 420,500 \mathrm{kWh} \text { electricity \& } \$ .05 / \mathrm{kWh} & \frac{21,025}{\$ 39,775}\end{array}$

3. Ground water heat pump

$$
\begin{array}{llr}
\text { Heating: } & 199,300 \mathrm{kWh} \text { electricity @ } \$ .05 / \mathrm{kWh} & 9,965 \\
\text { Cooling: } & 400,500 \mathrm{kWh} \text { electricity @ } \$ .05 / \mathrm{kWh} & 20,025 \\
\text { Well pump: } & 170,000 \mathrm{kWh} \text { electricity @ } \$ .05 / \mathrm{kWh} & \frac{8,500}{}
\end{array}
$$

The savings from the ground water heat pump would be greater if the waste heat from the heat pump were utilized as in the case of four pipe system, which is usually the case for large office buildings. The example represents a two pipe system, either heating or cooling.

The fastest growing segment of the market is ground water and earth coupled heat pumps used for space heating and cooling. It is estimated that almost 50,000 ground water systems and over 30,000 closed loop earth coupled systems $(2 / 3$ of these are vertical and $1 / 3$ horizontal pipe installations in the ground are being used in the U.S. In 1988 there will be over 8,000 ground water and 10,000 earth coupled systems installed, a 50 percent increase over last year. The popularity of these systems is due to the recent promotion by electric utility companies throughout the country, mainly in the midwest and east. It has national appeal since ground water temperatures down to $45^{\circ} \mathrm{F}$ can be used in heat pump systems.

Resorts and Pools

Geothermal energy used for swimming pools and spas is the earliest use of the resource. Natatoriums and large resorts developed at hot springs, located in both the eastern and western United states, were popular in the 1800's and were reminiscent of those in Europe. Many of these continue to be used today and in some cases elaborate facilities have been developed. For example, Fairmont Hot Springs Resort, a major new all-year resort near Butte, Montana is using a $640 \mathrm{ft}$ geothermal well (160\%) for space heating a 140-room hotel, mini-zoo, game room and restaurant in addition to large indoor and outdoor swimming pools. The resort also boasts a golf course, convention center and time-share condominiums.

The survey identified 114 resorts using geothermal energy, the largest being Paynes Fountain of Youth and Hot Springs state Park in Wyoming. 
Greenhouses

A number of commercial crops can be raised in greenhouses, making geothermal resources in cold climates particularly attractive. Crops include vegetables, flowers (potted and cut), house plants and tree seedlings.

Greenhouse heating can be accomplished by several methods; finned pipe, unit heaters, finned coils, soil heating, plastic tubing, cascading, and a combination of these methods (Rafferty, 1981). The use of geothermal energy for heating can reduce operating costs and allows operation in colder climates where commercial greenhouses would not normally be economical.

Economics of a geothermal greenhouse operation depend on many variables, such as the type of crop, climate, resource temperature, type of structure, etc. An example, is the raising of roses near Helena, Montana where using geothermal energy in a $75,500 \mathrm{ft}^{2}$ greenhouse reduces heating costs by 80 percent and overall costs by 35 percent.

Greenhouses are one of the fastest growing applications in the direct use industry. A number of the existing greenhouse systems are expanding. For example, Troy Hygro, Newcastle, Utah is building an additional 28 acres, which will result in a 100\% increase in the total U.S. utilization for greenhouses. Other systems expanding are Utah Roses, Bluffdale, Utah, Flint Greenhouses near Buhl, Idaho (doubling in size) and a new experimental facility and commercial space with a geothermal delivery system is being constructed by Lake county, California.

Troy-Hygro greenhouses will be the largest greenhouse energy user when the 28 acre facility is completed. Burgett Floral at Animas, New Mexico has developed about 13 acres and the state with the largest total use for greenhouses is Idaho with 14 sites in operation.

\section{Aquaculture}

Aquaculture involves the raising of freshwater or marine organisms in a controlled environment to enhance production rates. The principal species that are typically raised are aquatic animals such as catfish, bass, tilapia, sturgeon, shrimp, and tropical fish. The application temperature in fish farming depends on the species invloved. Typically, catfish grow in 4 to 6 months at 64 to $75 \mathrm{~F}$, trout in 4 to 6 months at 55 to $64 \mathrm{~F}$ and prawns in 6 to 9 months at 80 to $86^{\circ} \mathrm{F}$. The benefit of a controlled rearing temperature in aquaculture operations can increase growth rates by 50 to $100 \%$ and thus increase the number of harvests per year. Water quality and disease control are very important in fish farming. 
In the U.S. aquaculture projects using geothermal water exist in Arizona, Idaho, oregon, and California. Aquaculture is one of the fastest growing applications for using low-temperature geothermal energy. Recently, four locations in Arizona began raising catfish, tilapia and bass using geothermal fluids with temperatures ranging from 80 to $105^{\circ} \mathrm{F}$ and $\mathrm{a}$ large facility is planned for raising sturgeon at Brooks Warm Springs, Montana.

Aquaculture projects at the Hot Creek Hatchery near Mammoth Lakes, California and the Fish Breeders of Idaho, Buhl, Idaho are the largest aqcuaculture use sites.

Space and District Heating

District heating involves the distribution of heat (hot water or steam) from a central location, through a network of pipes to individual houses or blocks of buildings. The distinction between district heating and space heating systems, is that space heating usually invlolves one geothermal. well. per structure.

An important consideration in district heating projects is the thermal load density, or the heat demand divided by the ground area of the district. A high heat density is required to make district heating economically feasible, since the distribution network which transports the hot water to the consumers is expensive.

Geothermal district heating systems are capital intensive. The principal costs are initial investment costs for production and injection wells, downhole and circulation pumps, heat exchangers, pipelines and distribution network, "flowmeters, valves and control equipment, etc. Operating expenses, however are in comparison lower and consist of pumping power, system maintenance, control and management. The typical savings to consumers range from about 30 to $50 \%$ of the cost of natural gas.

A showcase of district heating developments are the two systems at Elko, Nevada. Elko Heat Company is a private company that has experienced considerable growth since it first began operating in 1982. The project started as a USDOE Program opportunity Notice demonstration project consisting of three buildings; a laundry, bank, and hotel/casino. The system has grown to include 14 commercial buildings and a sewage treatment plant. This was accomplished by offering a preliminary estimate of customer needs to retrofit, educating about the reliability of the system (down less than one day per year) and charging its customers about 50 percent the price of natural gas ( $\$ 1.00$ to $\$ 1.25$ per 1,000 gallon of geothermal fluids). The system supplies about 36.7 billion Btu/yr from its one geothermal well that produces $650 \mathrm{gpm}$ at $170^{\circ} \mathrm{F}$. The company has doubled the length of its delivery piping system and has reached the demand point where it will be necessary to drill a second well. 
The Elko County school District in conjunction with the Elko Hospital, has been servicing the High School, Jr. High (heat pump system), gymnasium, school administrative offices, hospital, convention center, city hall and the municipal pool for about two years. One of the most impressive aspects of this system is the $100^{\circ}$ temperature drop through the closed loop servicing the buildings from two plate heat exchangers in parallel. An injection well was drilled, but is not used due to concern of contamination of domestic aquifers. Disposal of geothermal fluids is to percolation ponds, storm drains, and a golf course irrigation system.

Others that have experienced considerable growth are san Bernardino, California system and Warren Properties at Reno, Nevada (doubling is size). When completed, Mammoth Lakes district heating will be the largest development in the country. This is followed by the Litchfield correctional Center at Susanville, California and the two systems in Boise, Idaho, the downtown commercial system and the Boise Warm Springs residential system.

The Peppermill Casino, Reno, Nevada has the largest space and domestic hot water use followed by the 500 individual homes that utilize downhole heat exchangers in Klamath Falls, oregon.

The potential for geothermal district heating in the United states is very large. An inventory identifies a total of 1,277 hydrothermal sites within five miles of 373 cities in eight Western states, with a combined population of $6,720,000$ persons. The combined heat load for all cjties (exclusive of industrial loads) is estimated at $1.3 \times 10^{14}$ Btu/yr (Allen, 1980). Currently 23 geothermal district heating systems are operating $\left(700 \times 10^{5}\right.$ $\mathrm{Btu} / \mathrm{Yr}$ ) and 26 planned projects should increase the annual energy use by 1,690 billion Btu per year.

\section{CONCLUSIONS}

The contained heat energy beneath the United states could, in theory, provide most of the future low temperature energy needs of this Nation. The actual contribution will be determined by the effort - time, people, and funding - devoted to a broad research, development, and demonstration program with participation by Federal, state, and local governments in cooperation with industry, universities, laboratories and the American people.

The United states direct use industry is and will continue to experience a significant growth rate. The largest growth should continue to occur in the use of groundwater heat pumps, and aquaculture, greenhousing and district heating will add to the expansion of the industry. 
Table 3

Geothermal Direct Use Projects in the United States

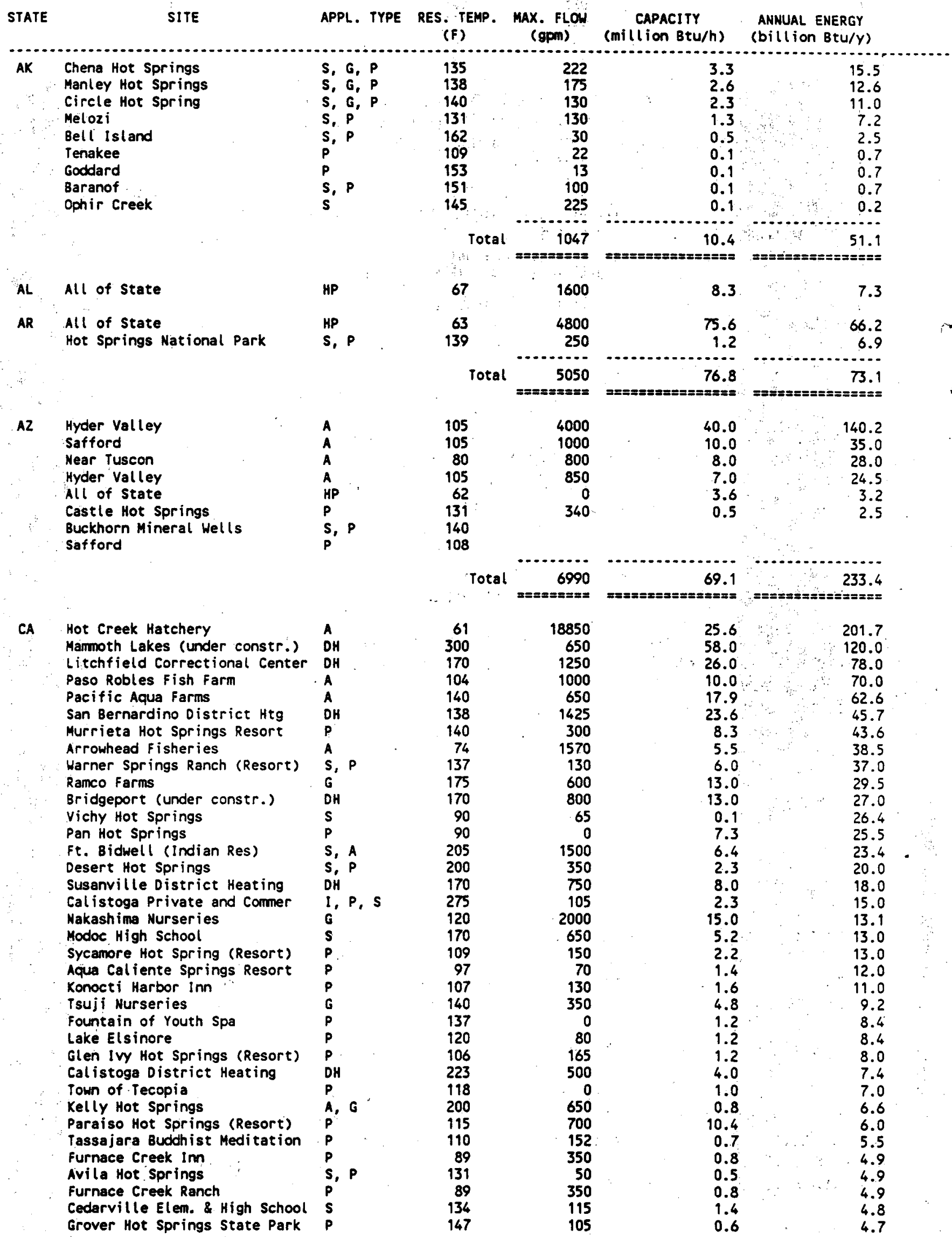

Appl. Type Key: $S=$ Space Heating, $\mathrm{OH}=0$ istrict Heating, HP $=$ Heat Pum, $G=$ Greenhouse, $I=$ Industrial Process, $A=$ Aquacul ture, $P=$ Swiming Pools 
Table 3

Geothermal Direct Use Projects in the United States

\begin{tabular}{|c|c|}
\hline STATE & SITE \\
\hline$C A$ & $\begin{array}{l}\text { Wilbur Hot Springs } \\
\text { Campbell Hot Springs } \\
\text { Whitmore Hot Springs } \\
\text { Harbin Hot Springs } \\
\text { Indian Springs School } \\
\text { Esalen Institute } \\
\text { California Hot Springs } \\
\text { Cal isoga High School } \\
\text { Keough Hot Springs } \\
\text { Palm Springs Spa } \\
\text { La Vide Mineral Springs } \\
\text { Orr Hot Springs } \\
\text { Bashfords Hot Mineral Spa } \\
\text { Hhite Suphur Springs } \\
\text { San Luis Bay Estates } \\
\text { Surprise Valley Hospial } \\
\text { LDS Church } \\
\text { Shoshone Motel \& Trailer Park } \\
\text { Aqua Caliente County Park } \\
\text { Drakesbad Guest Ranch } \\
\text { Imperial Sea View Hot Springs } \\
\text { Mono Hot Springs } \\
\text { Indian Valley Hospital } \\
\text { Matilija Hot Springs } \\
\text { Democrat Hot Springs Resort } \\
\text { San Juan Hot Springs } \\
\text { Big Bend Preventorium } \\
\text { Lake Elsinore Dist. Heating } \\
\text { Aqua Farms International } \\
\text { Jacumba Hot Springs Health Spa } \\
\text { Lake County Ag Park } \\
\text { Sal ine Valley Hot Springs } \\
\text { Reds Meadow Hot Springs } \\
\text { Miracle Hot Spring } \\
\text { Arrowhead Hot Springs } \\
\text { Twenty-Nine Palms (City of) } \\
\text { Cal ifornia Pines } \\
\text { Hot Creek } \\
\text { Tecopia Hot Springs } \\
\text { Fales Hot Springs } \\
\text { Modesto Memorial Hospital } \\
\text { Nevares Springs }\end{array}$ \\
\hline
\end{tabular}

APPL. TYPE RES. TEMP. MAX. FLOW CAPACITY

ANNUAL ENERGY

(F) (gpm)
(million Btu/h) (billion Btu/y)

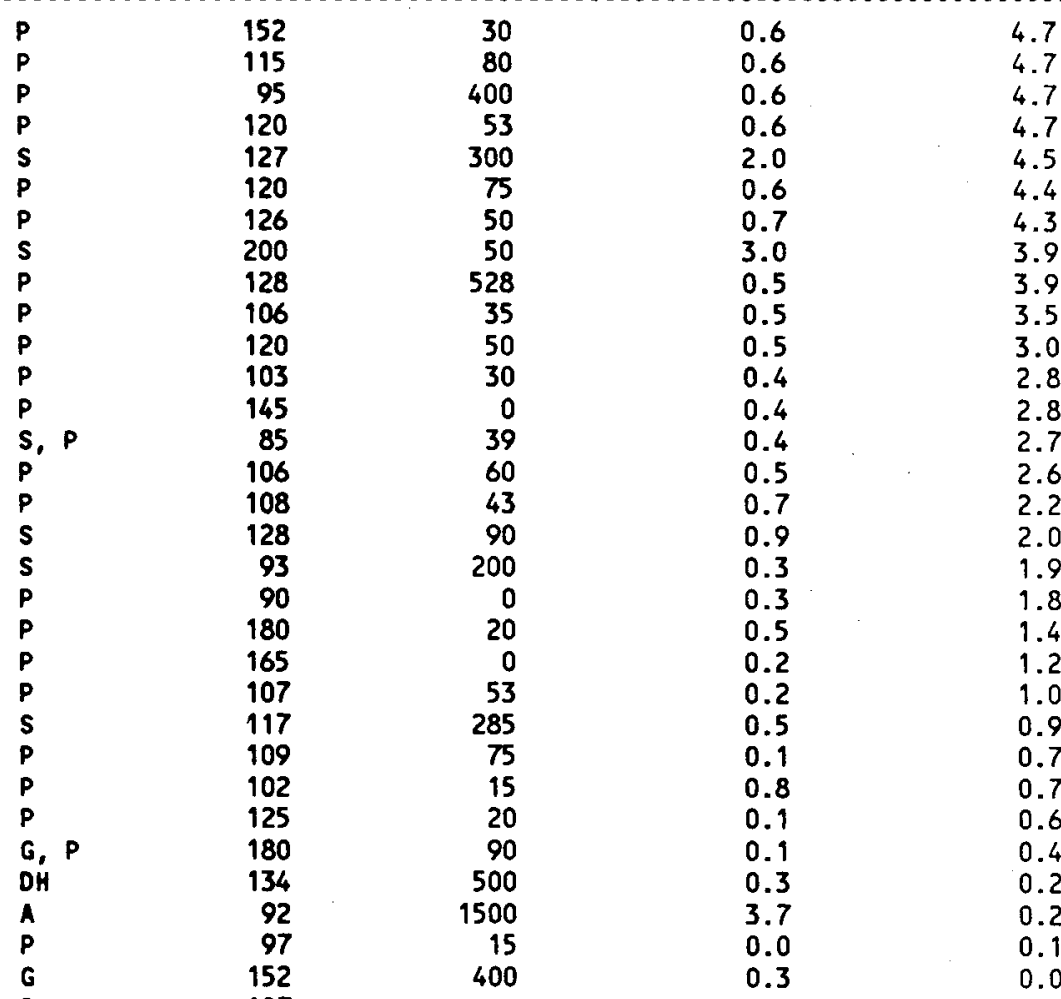

$\begin{array}{llr}\text { Sand Dunes Hot Spring } & \text { A } & 118 \\ \text { Glenwood Hot Springs Hotel } & \text { P, S } & 130 \\ \text { Roaring Judy Fish Hatchery } & \text { A } & 65 \\ \text { Pagosa Springs Oistrict Htg. } & \text { DH } & 149 \\ \text { Jones Splashland } & \text { P } & 106 \\ \text { The Spa Motel } & \text { S, P } & 123 \\ \text { Pagosa Springs Private Wells } & \text { S } & 120 \\ \text { Haunita Hot Springs Ranch } & \text { S, G, P } & 175 \\ \text { All of State } & \text { HP } & 52 \\ \text { Steamboat Springs Heal th \& Rec } & \text { P } & 104 \\ \text { Ouray Municipal Pool } & \text { S, P } & 156 \\ \text { Old Wright Well } & \text { G } & 160 \\ \text { Jump Steady Resort } & \text { S, P } & 119 \\ \text { Cottonwood Hot Springs } & \text { S } & 119 \\ \text { Valley View Hot Springs } & \text { P } & 99 \\ \text { 4 UR Guest Ranch } & \text { P } & 135 \\ \text { Salida Hot Springs(Poncha Spr) } & \text { S, P } & 160 \\ \text { Hot Sulphur Springs } & \text { S, P } & 111 \\ \text { Indian Springs Resort } & \text { P } & 115 \\ \text { Wiesbaden Motel \& Health Res. } & \text { S, P } & 117 \\ \text { Pinkerton Hot Springs } & \text { S, P } & 93 \\ \text { Mount Princeton Hot Springs } & \text { P, DHW } & 132 \\ \text { Twin Peaks Motel } & \text { S, P } & 110\end{array}$

15

$\begin{array}{lll}P & 115 & 15 \\ S, P & 122 & 15\end{array}$

S. $P \quad 190 \quad 500$

140

OHW 100

P $\quad 200 \quad 4000$

$S, P \quad 108 \quad 200$

$S, P \quad 142 \quad 300$

DHW 104

Total

47583

905

5.0

314.0

1107.2

$== \pm== \pm= \pm=$

$=============$

13.3

9.0

81.2

500
2263

2263
1100

800

310

220

675

100

0

140
180

180
120

90

90
10

180

180
50

200

60

60

60

77

175

15
4.7

4.7

4.7

4.5
4.4

4.3

3.9

3.9
3.5

3.0

2.8

2.7

2.6

2.2

1.9

1.2

1.0

0.7

0.7

.6

0.2

0.1
0.0

Appl. Type Key: $S=$ Space Heating, $D H=$ District Heating, $H P=$ Heat Pump, $G=$ Greenhouse, $I=$ Industrial Process, $A=$ Aquacul ture, $P=$ Swiming Pools 
Table 3

Geothermal Direct Use Projects in the United States

\begin{tabular}{|c|c|c|c|c|c|c|}
\hline STATE & SITE & APPL. TYPE & $\begin{array}{l}\text { RES. TEMP. } \\
\text { (F) }\end{array}$ & $\begin{array}{l}\text { MAX. FLOU } \\
\text { (gpm) }\end{array}$ & $\begin{array}{c}\text { CAPACITY } \\
\text { (million Btu/h) }\end{array}$ & $\begin{array}{l}\text { ANNUAL ENERGY } \\
\text { (billion } B \text { tu/y) }\end{array}$ \\
\hline $\begin{array}{l}C O \\
\therefore\end{array}$ & $\begin{array}{l}\text { Box Canyon Motel } \\
\text { Mount Princeton Area } \\
\text { Heal th Spa } \\
\text { Lope Hot Springs } \\
\text { Cement Creek Ranch } \\
\text { Dunton Hot Springs } \\
\text { Trip Hot Springs } \\
\text { Glenwood Springs Vapor Caves } \\
\text { Ouray District Heating } \\
\text { Canon City Area }\end{array}$ & $\begin{array}{l}\text { S; } P \\
\text { S, P } \\
\text { S, P } \\
\text { P } \\
\text { P } \\
\text { P } \\
\text { G, P } \\
\text { P } \\
\text { DH } \\
\text { S }\end{array}$ & $\begin{array}{r}110 \\
132 \\
114 \\
132 \\
78 \\
107 \\
111\end{array}$ & $\begin{array}{r}15 \\
6 \\
20 \\
70 \\
25\end{array}$ & $\begin{array}{l}0.2 \\
0.3 \\
0.1 \\
0.1 \\
0.1 \\
0.1\end{array}$ & $\begin{array}{l}1.1 \\
0.6 \\
0.4 \\
0.4 \\
0.4 \\
0.3\end{array}$ \\
\hline & & & Total & $========8$ & 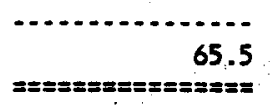 & 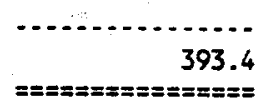 \\
\hline DE & All of State & HP & 57 & 4000 & 20.5 & 35.9 \\
\hline FL & $\begin{array}{l}\text { All of State } \\
\text { Patrick Air Force Base }\end{array}$ & $\begin{array}{l}\text { HP } \\
\text { HP }\end{array}$ & $\frac{75}{72}$ & $\begin{array}{r}160000 \\
8000\end{array}$ & $\begin{array}{r}800.0 \\
39.6\end{array}$ & $\begin{array}{l}700.8 \\
138.8\end{array}$ \\
\hline & & & Total & $==== \pm== \pm=$ & 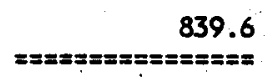 & $\Rightarrow==============$ \\
\hline GA & $\begin{array}{l}\text { All of State } \\
\text { Roosevelt Warm Springs Inst. }\end{array}$ & $\begin{array}{l}\text { HP } \\
S, H P, P\end{array}$ & $\begin{array}{l}67 \\
88\end{array}$ & $\begin{array}{r}4800 \\
800\end{array}$ & $\begin{array}{r}24.5 \\
1.1\end{array}$ & $\begin{array}{r}21.4 \\
3.4\end{array}$ \\
\hline & & & Total & $========0$ & 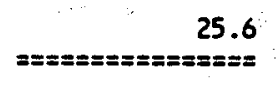 & $===============0$ \\
\hline HI & Community Geothermal Tech Prog & 1 & 347 & 110 & & \\
\hline IA & All of State & HP & 52 & 2400 & 23.4 & 41.0 \\
\hline 10 & 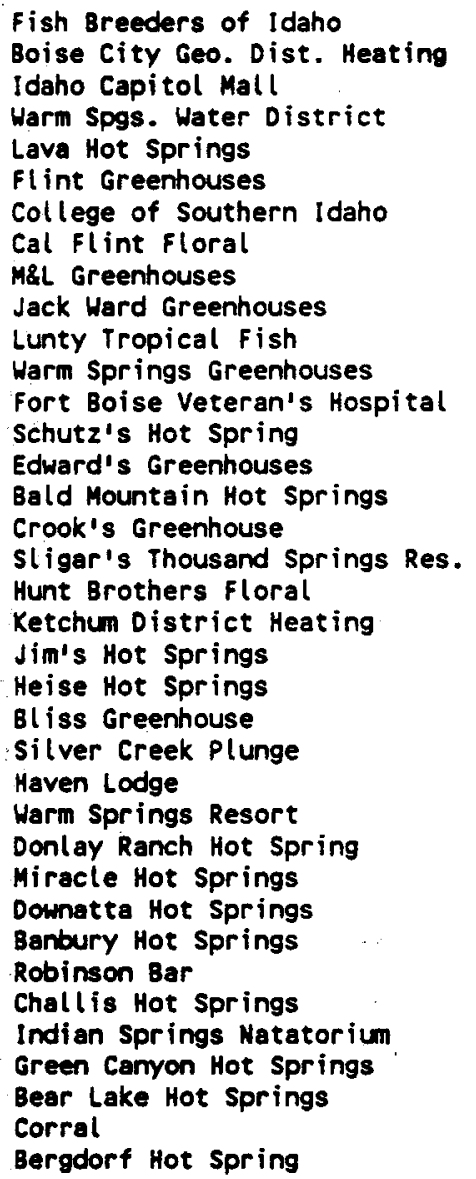 & $\begin{array}{l}A \\
D H \\
D H \\
D H \\
S, P \\
G \\
H P \\
G \\
G \\
G \\
A \\
G, S \\
S \\
S, P \\
G \\
P \\
G \\
P \\
G, S \\
D H \\
P \\
P \\
G, S, A \\
P \\
P \\
P \\
G \\
S, P \\
P \\
S, P \\
P \\
P \\
P \\
G, P \\
P \\
S, G \\
S, P\end{array}$ & $\begin{array}{r}90 \\
170 \\
162 \\
170 \\
112 \\
112 \\
102 \\
160 \\
112 \\
138 \\
90 \\
180 \\
162 \\
176 \\
117 \\
168 \\
194 \\
200 \\
117 \\
158 \\
151 \\
120 \\
151 \\
101 \\
148 \\
110 \\
130 \\
139 \\
112 \\
131 \\
135 \\
127 \\
90 \\
118 \\
115 \\
167 \\
113\end{array}$ & $\begin{array}{r}6200 \\
700 \\
750 \\
700 \\
1800 \\
570 \\
1220 \\
490 \\
460 \\
390 \\
400 \\
240 \\
300 \\
600 \\
265 \\
2 \\
140 \\
140 \\
200 \\
1027 \\
60 \\
80 \\
60 \\
110 \\
50 \\
100 \\
72 \\
50 \\
80 \\
80 \\
40 \\
40 \\
120 \\
80 \\
40 \\
31 \\
162\end{array}$ & $\begin{array}{l}24.8 \\
17.5 \\
15.8 \\
12.3 \\
3.0 \\
9.1 \\
8.2 \\
7.5 \\
7.4 \\
6.9 \\
2.0 \\
6.0 \\
6.0 \\
3.2 \\
4.9 \\
2.8 \\
4.0 \\
1.3 \\
3.0 \\
3.0 \\
0.8 \\
1.0 \\
1.2 \\
0.9 \\
0.5 \\
0.9 \\
1.2 \\
0.7 \\
0.6 \\
0.8 \\
0.6 \\
0.5 \\
0.6 \\
0.6 \\
0.3 \\
0.6 \\
0.4\end{array}$ & $\begin{array}{l}174.0 \\
42.8 \\
31.7 \\
30.0 \\
20.2 \\
19.9 \\
18.0 \\
16.4 \\
16.2 \\
15.1 \\
14.0 \\
13.1 \\
12.1 \\
10.8 \\
10.7 \\
10.0 \\
9.0 \\
7.7 \\
6.6 \\
6.6 \\
5.3 \\
4.4 \\
3.7 \\
3.6 \\
3.5 \\
3.2 \\
3.2 \\
2.9 \\
2.5 \\
2.3 \\
2.3 \\
2.2 \\
2.1 \\
2.0 \\
1.3 \\
1.2 \\
1.2\end{array}$ \\
\hline
\end{tabular}

Appl. Type Key: $s=$ Space Heating, $D H=$ District Heating, HP = Heat Pump, $G=$ Greenhouse, $I=$ Industrial Process, $A=$ Aquacul ture, $P=$ Swiming Pools 
Table 3

Geothermal Direct Use Projects in the United States

\begin{tabular}{|c|c|c|c|c|c|c|}
\hline STATE & SITE & APPL. TYPE & $\begin{array}{l}\text { RES. TEMP. } \\
\text { (F) }\end{array}$ & $\begin{array}{l}\text { MAX. FLOW } \\
\quad(\mathrm{gpm})\end{array}$ & $\begin{array}{c}\text { CAPACITY } \\
\text { (million Btu/h) }\end{array}$ & $\begin{array}{l}\text { ANNUAL ENERGY } \\
\text { (billion } \mathrm{Btu} / \mathrm{Y} \text { ) }\end{array}$ \\
\hline \multirow[t]{2}{*}{10} & $\begin{array}{l}\text { LDS Church } \\
\text { Del Rio Hot Springs } \\
\text { Riggins Hot Springs } \\
\text { Express Farms } \\
\text { Weiser Hot Springs } \\
\text { Givens Hot Springs } \\
\text { Stanley Dist. Htg. (proposed) } \\
\text { Twin Springs Resort }\end{array}$ & $\begin{array}{l}\text { S } \\
\text { S, G } \\
G, S \\
G \\
G, S, P \\
P \\
\text { DH } \\
S, P\end{array}$ & $\begin{array}{r}93 \\
200 \\
113 \\
99 \\
158 \\
120 \\
140 \\
180\end{array}$ & $\begin{array}{r}80 \\
20 \\
50 \\
47 \\
15 \\
650 \\
290\end{array}$ & $\begin{array}{l}0.4 \\
0.4 \\
0.4 \\
0.4 \\
0.3 \\
0.2 \\
0.0\end{array}$ & $\begin{array}{l}0.9 \\
0.9 \\
0.8 \\
0.8 \\
0.7 \\
0.5 \\
0.0\end{array}$ \\
\hline & $\therefore$ & & Total & $=========$ & $====z=x==== \pm===0$ & $===============$ \\
\hline IL & All of State & HP & 54 & 8400 & 100.8 & 176.6 \\
\hline \multirow[t]{2}{*}{ IN } & $\begin{array}{l}\text { All of State } \\
\text { Corporate Square }\end{array}$ & $\begin{array}{l}\text { HP } \\
\text { HP }\end{array}$ & $\begin{array}{l}54 \\
55\end{array}$ & $\begin{array}{r}32000 \\
0\end{array}$ & $\begin{array}{r}187.2 \\
4.2\end{array}$ & $\begin{array}{r}328.0 \\
7.4\end{array}$ \\
\hline & & & Total & $=========$ & $===============$ & $==============0$ \\
\hline \multirow[t]{2}{*}{ KS } & $\begin{array}{l}\text { All of State } \\
\text { Elementary Schools (3) }\end{array}$ & $\begin{array}{l}\text { HP } \\
\text { HP }\end{array}$ & $\begin{array}{l}57 \\
59\end{array}$ & $\begin{array}{r}1600 \\
440 \\
\ldots . . .\end{array}$ & $\begin{array}{l}8.0 \\
4.7 \\
-.-\end{array}$ & $\begin{array}{r}14.0 \\
3.3 \\
-. .\end{array}$ \\
\hline & & & Total & $=========$ & $================$ & $======= \pm=== \pm= \pm== \pm$ \\
\hline KY & All of State & HP & 59 & 4200 & 88.2 & 154.5 \\
\hline LA & All of state & HP & 69 & 0 & 90.0 & 78.8 \\
\hline MA & English High School & HP & 55 & 80 & 0.4 & 0.9 \\
\hline MD & All of State & HP & 57 & 6400 & 46.8 & 82.0 \\
\hline MI & All of state & HP & 47 & 28000 & 135.0 & 354.8 \\
\hline MN & All of State & HP & 45 & 6800 & 36.0 & 94.6 \\
\hline MO & All of State & HP & 57 & 8000 & 54.0 & 47.3 \\
\hline \multirow[t]{2}{*}{ MS } & $\begin{array}{l}\text { All of State } \\
\text { Mississippi Power Co. } \\
\text { Commercial Buildings }\end{array}$ & $\begin{array}{l}\text { HP } \\
\text { HP } \\
\text { HP }\end{array}$ & $\begin{array}{l}67 \\
69 \\
69\end{array}$ & $\begin{array}{r}800 \\
0 \\
0\end{array}$ & $\begin{array}{l}4.0 \\
1.1 \\
1.0\end{array}$ & $\begin{array}{l}3.5 \\
0.9 \\
0.9\end{array}$ \\
\hline & & & Total & $========$ & $===============0$ & $================$ \\
\hline MT & $\begin{array}{l}\text { Secondary Oil Recovery } \\
\text { High Country Rose Greenhouses } \\
\text { White Sulfur Springs } \\
\text { Warm Springs State Hospital } \\
\text { Fairmont Hot Springs Resort } \\
\text { Lolo Hot Springs } \\
\text { Bozeman Hot Springs } \\
\text { Broadwater Athletic Club \& HS } \\
\text { Chico Hot Springs } \\
\text { Boulder Hot Springs } \\
\text { Jackson Hot Springs Lodge } \\
\text { Medicine Hot Springs } \\
\text { Sleeping Child Hot Springs } \\
\text { Barkell's Hot Springs } \\
\text { New Biltmore Hot Springs } \\
\text { Emis Hot Springs } \\
\text { Camas Hot Springs } \\
\text { Hillbrook Nursing Home } \\
\text { Brooks Harm Springs }\end{array}$ & $\begin{array}{l}\text { I } \\
G \\
\text { DH } \\
\text { S } \\
\text { S, P } \\
\text { S, P } \\
\text { S,P } \\
\text { S, P } \\
\text { P } \\
\text { S, P, G } \\
\text { S, P } \\
\text { P } \\
P \\
P \\
P \\
1 \\
P \\
\text { S, P } \\
\text { P }\end{array}$ & $\begin{array}{l}200 \\
151 \\
136 \\
154 \\
160 \\
111 \\
131 \\
153 \\
113 \\
169 \\
136 \\
113 \\
113 \\
161 \\
127 \\
181 \\
104 \\
133 \\
70\end{array}$ & $\begin{array}{r}9000 \\
207 \\
460 \\
90 \\
200 \\
180 \\
977 \\
100 \\
320 \\
500 \\
259 \\
100 \\
530 \\
150 \\
26 \\
100 \\
24 \\
100 \\
72000\end{array}$ & $\begin{array}{r}542.7 \\
8.4 \\
5.3 \\
1.9 \\
3.0 \\
1.8 \\
2.0 \\
1.4 \\
0.8 \\
1.0 \\
0.5 \\
0.6 \\
0.7 \\
0.7 \\
0.3 \\
0.8 \\
0.2 \\
0.2 \\
0.0\end{array}$ & $\begin{array}{r}3803.0 \\
33.1 \\
16.2 \\
14.6 \\
14.5 \\
12.6 \\
5.8 \\
5.6 \\
4.6 \\
4.4 \\
2.9 \\
2.6 \\
2.5 \\
2.2 \\
1.8 \\
1.7 \\
0.7 \\
0.6 \\
0.0\end{array}$ \\
\hline & & & Total & $=========$ & $=== \pm== \pm=========$ & =============== \\
\hline
\end{tabular}

Appl. Type Key: $s=$ Space Heating, $D H=$ District Heating,

$H P=$ Heat Pump, $G=$ Greenhouse, $I=$ Industrial Process,

$A=$ Aquacult ture, $P=$ Swiming Pools 
Table 3

Geothermal Direct Use Projects in the United States

$08 / 23 / 88$

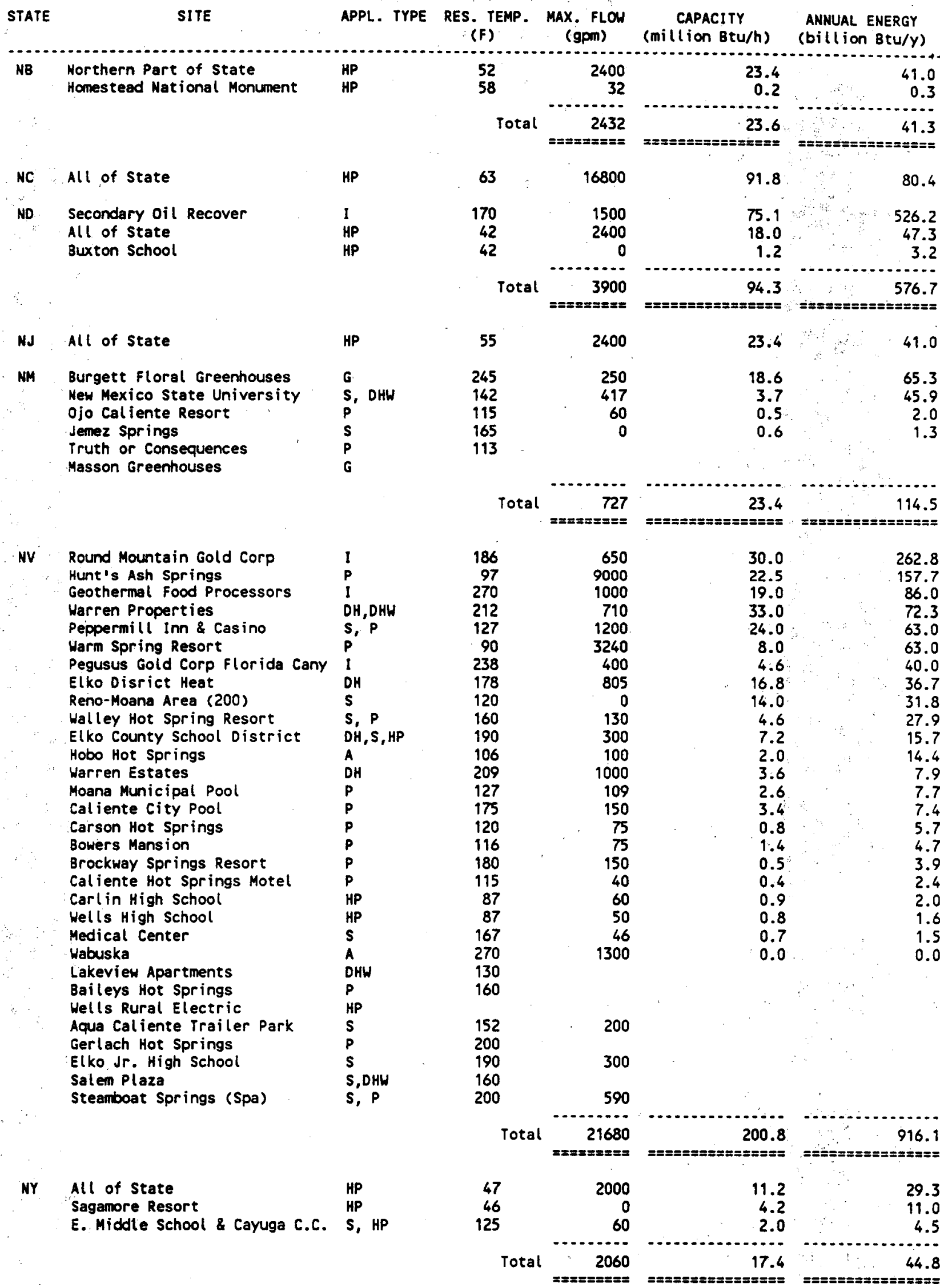

Appl. Type Key: $s=$ Space Heating, $D H=$ District Heating, $H P=$ Heat Pump, $G=$ Greenhouse, $I=$ Industrial Process, $A=$ Aquaculture, $P=$ Swimming Pools 
Table 3

Geothermal Direct Use Projects in the United States

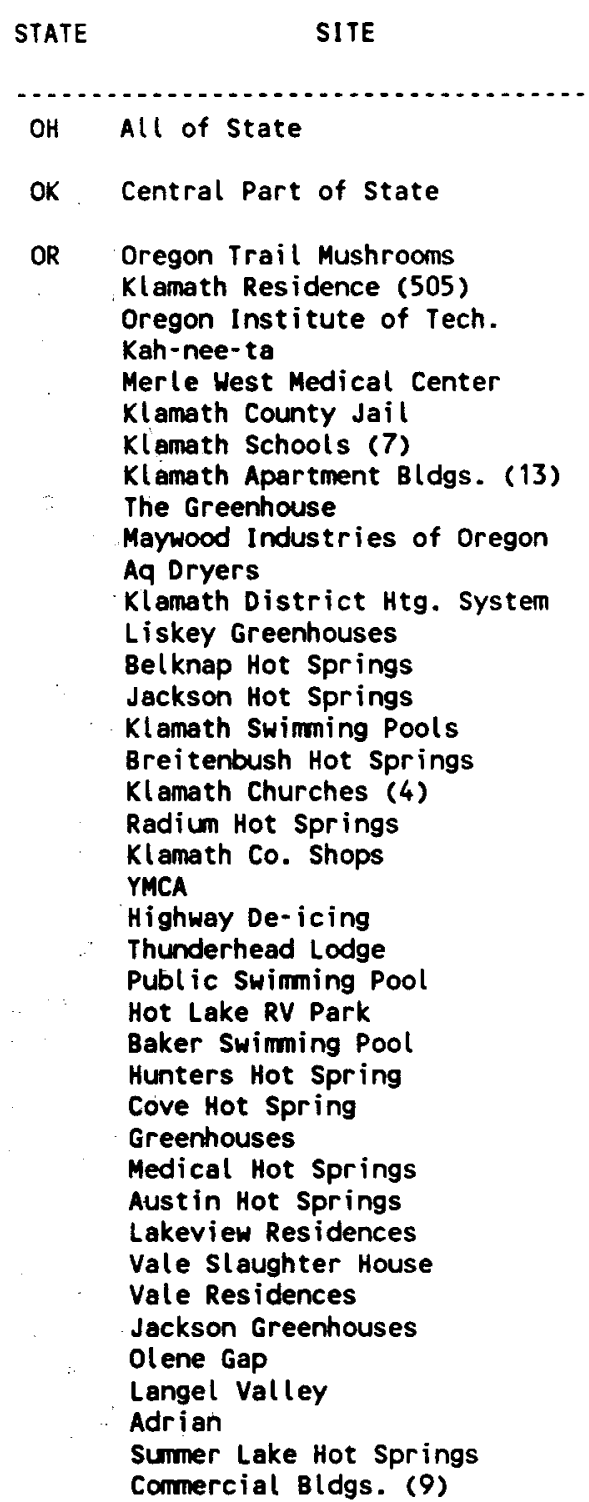

APPL. TYPE RES. TEMP. MAX. FLOW

CAPACITY

ANNUAL ENERGY (F) (gpm)

(million Btu/h)

(billion Btu/Y)

HP

53

HP

I 235

I
DH
$P$.

P, DHW

S

S

S

G

S

DH

G

P

P,

S,

$S$

I,

HP

$\stackrel{H P}{P}$

$S, P$

$P$, HP

G

S,

P

S

S

G

S

I

HṔ

HP 109

62

235
200

200
192

126

191

150

180

180
220

118

200

210

199

160

111
110

180

212

190

136

118
147

147
190

190
68

180

190

75
202

202
108

195

140

186

190

150

185

111

189

147

168

109

25600

240

275

0
620

620
450

325

700

0
0

700

450

60

720

500

50
264

0

900

300

113

120

130

20

830

200

78
226

226
40

100

100
250

50

20

24

100

300

20

150
20
129.6

$$
1.2
$$

20.6

20.2

16.8

4.5

10.5

10.5

8.7

6.5

4.7

3.0

3.0

2.8

2.7

0.9

0.7

1.1

1.2

1.7

0.6

1.6

1.4

0.4

1.2

0.8

0.9

0.6

0.8

0.7

0.6

0.5

0.2

0.4

0.3

0.3

0.3

0.1

0.1

0.0
227.0

1.0

54.1

44.2

38.2

27.6

23.9

23.0

19.8

14.2

8.8

6.8

6.5

6.2

5.9

5.5

4.4

4.3

3.9

3.9

3.6

3.6

3.1

2.5

2.3

1.8

1.8

1.8

1.7

1.4

1.3

1.1

1.0

0.9

0.7

0.7

0.5

0.1

0.1

0.0

PA All of state

Factory at Masontown

$\begin{array}{ll}H P & 50 \\ \text { HP } & 5\end{array}$

131.9

331.2

$========$

$\begin{array}{lr}50 & 12000 \\ 52 & 0 \\ & \ldots . \\ \text { Total } & 12000 \\ & ========\end{array}$

$\begin{array}{rr}67 & 11200 \\ 170 & 1000 \\ 87 & 10340\end{array}$

10340
2000

$\begin{array}{rr}47 & 2000 \\ 73 & 935\end{array}$

$155 \quad 300$

$108 \quad 385$

154

250

152

170

Total 15380

==ニ==ニ===

92.5

68.4

119.8

1.0

1.7

69.4

121.5

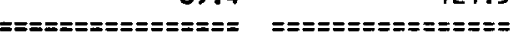

61.2

53.6

47.8

5.2

13.5

7.7

8.4

5.6

4.3

334.9

36.2

35.5

20.6

17.8

11.4

9.3

465.7

840

17.6

15.4

Appl. Type Key: $S=$ Space Heating, $D H=$ District Heating, HP $=$ Heat Pump, $G=$ Greenhouse, $I=$ Industrial Process,

$A=$ Aquacul ture, $P=$ Swimming Pools 
Table 3

Geothermal Direct Use Projects in the United States

STATE

SITE

APPL. TYPE RES. TEMP. MAX. FLOW

CAPACITY

ANNUAL ENERGY

(F) (gpm) (million Btu/h) (billion Btu/y)

TX. All of state

Cotulla High School

Stacy Park Pool

$\begin{array}{lr}\text { HP } & 67 \\ \mathbf{S} & 109 \\ \mathbf{P} & 96 \\ \text { G, A } & 126 \\ \text { S } & \end{array}$

$\begin{array}{rrrr}67 & 3600 & 147.9 & 129.6 \\ 109 & 600 & 9.9 & 13.0 \\ 96 & 250 & 0.9 & 5.5\end{array}$

Navarro College

Marlin Hospital

UT Tryo Hygro (28a. under constr) G LDS Office Building

Utah Ross

Como Springs Resort

Utah Roses

Crystal Hot Springs

Utah State Prison

Saratoga Springs Resort

Monroe Hot Springs

Belmont Springs

Veyo Resort

$P$ ah Tempe

The Homestead

Mountain Spa Resort

G 230

HP 60

$G \quad 124$

P 82

$G$

P, A

s, DHW

$S, P$

P, S, G

$P$

$P, S$

P

26

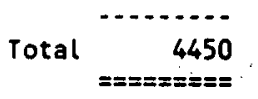

$=2=2=0=0$

246

1200

$82 \quad 540$

$190 \quad 400$

$\begin{array}{rr}140 & 1800 \\ 178 & 500\end{array}$

$120 \quad 150$

$164 \quad 600$

131.3600

$98 \quad 120$

$108 \quad 10000$

95

115

Total

1268

Total

2048

$=== \pm====$

HP 59

All of State
Homestead Resort

$S, H P, P$

59
104

$====\begin{array}{r}4000 \\ 223 \\ 4223 \\ \ldots==\end{array}$

$55 \quad 850$

$84 \quad 600$

$76 \quad 700$

$60 \quad 500$

$55 \quad 400$

$70 \quad 180$

128

80

70

56

80

Nazarene Church

Grant Co. PUD

Red Cross Bldg.

Uelikamje, Moore \& Shone, Inc.

Elephant House at $\mathbf{2 0 0}$

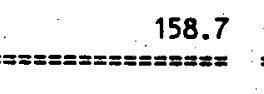

138.0

27.0

20.4

1.1

10.4

3.5

7.0

2.0

1.0

1.5

1.0

0.4

0.1

0.5

213.9

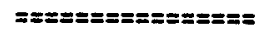

36.0

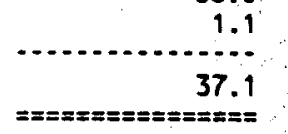

63.1

2.9

66.0

6.8

3.7

3.6

3.1

3.0

1.8

0.5

0.2

0.1

0.0

13.8

8.4

7.3

6.6

6.0

3.7

1.2

0.2

0.2

0.0

$$
\text { Total }
$$
3300
$=$

-

22.8

47.4

$\begin{array}{rr} & \\ 46 & 13600 \\ 200 & 8300 \\ 135 & 3120 \\ 125 & 1220 \\ 114 & 450 \\ 140 & 300 \\ 118 & 120 \\ 78 & 100 \\ 111 & 150 \\ 102 & 250 \\ 102 & 208 \\ 97 & 400 \\ 104 & 100 \\ 144 & 37\end{array}$

WI All of State

Wy Secondary Oil Recovery

Hot Springs State Park

Paynes Fountain of Youth RV PK

The Saratoga Inn

Huckelberry Hot Springs

Hobo Pool

Jackson National Fish Hatchery

Chief Washakie Plunge

Cody Athletic Club

Bronze Boot Spa

DeMaris Hot Springs

Astoria Mineral Hot Springs

Auburn Hot Spring

$H P$
1
$P$
$P$
$P$
$P, I$
$P$
$A$
$P$
$P$
$P$
$P$
$P$
$P$

Appl: Type Key: $s=$ Space Heating, $\mathrm{OH}=$ District Heating,

$H P=$ Heat Pump, $G=$ Greenhouse, $I=$ Industrial Process,

$A=$ Aquacul ture, $P=$ Swimming Pools 
Table 3

Geothermal Direct Use Projects in the United States

\begin{tabular}{|c|c|c|c|c|c|c|}
\hline STATE & SITE & APPL. TYPE & $\begin{array}{l}\text { RES. TEMP. } \\
\text { (F) }\end{array}$ & $\begin{array}{l}\text { MAX. FLOW } \\
\text { (gpm) }\end{array}$ & $\begin{array}{c}\text { CAPACITY } \\
\text { (million Btu/h) }\end{array}$ & $\begin{array}{l}\text { ANNUAL ENERGY } \\
\text { (billion } B \text { tu/y) }\end{array}$ \\
\hline \multirow[t]{2}{*}{ WY } & $\begin{array}{l}\text { Jackal ope Plunge } \\
\text { Frank Nixon Residence } \\
\text { Steele Hot Springs } \\
\text { Countryman Well } \\
\text { Van Norman residence } \\
\text { I-80 16th St. off ramp } \\
\text { East Grand St. Bridge }\end{array}$ & $\begin{array}{l}\text { P } \\
P \\
P \\
G, A \\
\text { S } \\
I \\
I\end{array}$ & $\begin{array}{r}86 \\
118 \\
102 \\
98 \\
124 \\
47 \\
47\end{array}$ & $\begin{array}{r}800 \\
35 \\
25 \\
500 \\
0 \\
0 \\
0\end{array}$ & $\begin{array}{l}0.4 \\
0.3 \\
0.2 \\
0.4 \\
0.0 \\
0.9 \\
0.2\end{array}$ & $\begin{array}{l}2.7 \\
2.1 \\
1.8 \\
1.6 \\
0.6\end{array}$ \\
\hline & & & Total & $========$ & 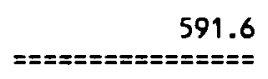 & $================$ \\
\hline & & & Grand Total & $=========$ & 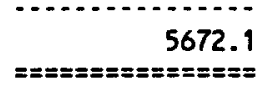 & 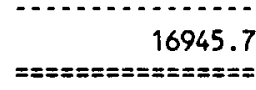 \\
\hline
\end{tabular}

Appl. Type Key: $S=$ Space Heating, $D H=$ District Heating,

$H P=$ Heat Pump, $G=$ Greenhouse, $I=$ Industrial Process,

$A=$ Aquacul ture, $P=$ Swiming Pools 
Table 4

Heat Pump Cooling Load Sites in the United States

$08 / 23 / 88$

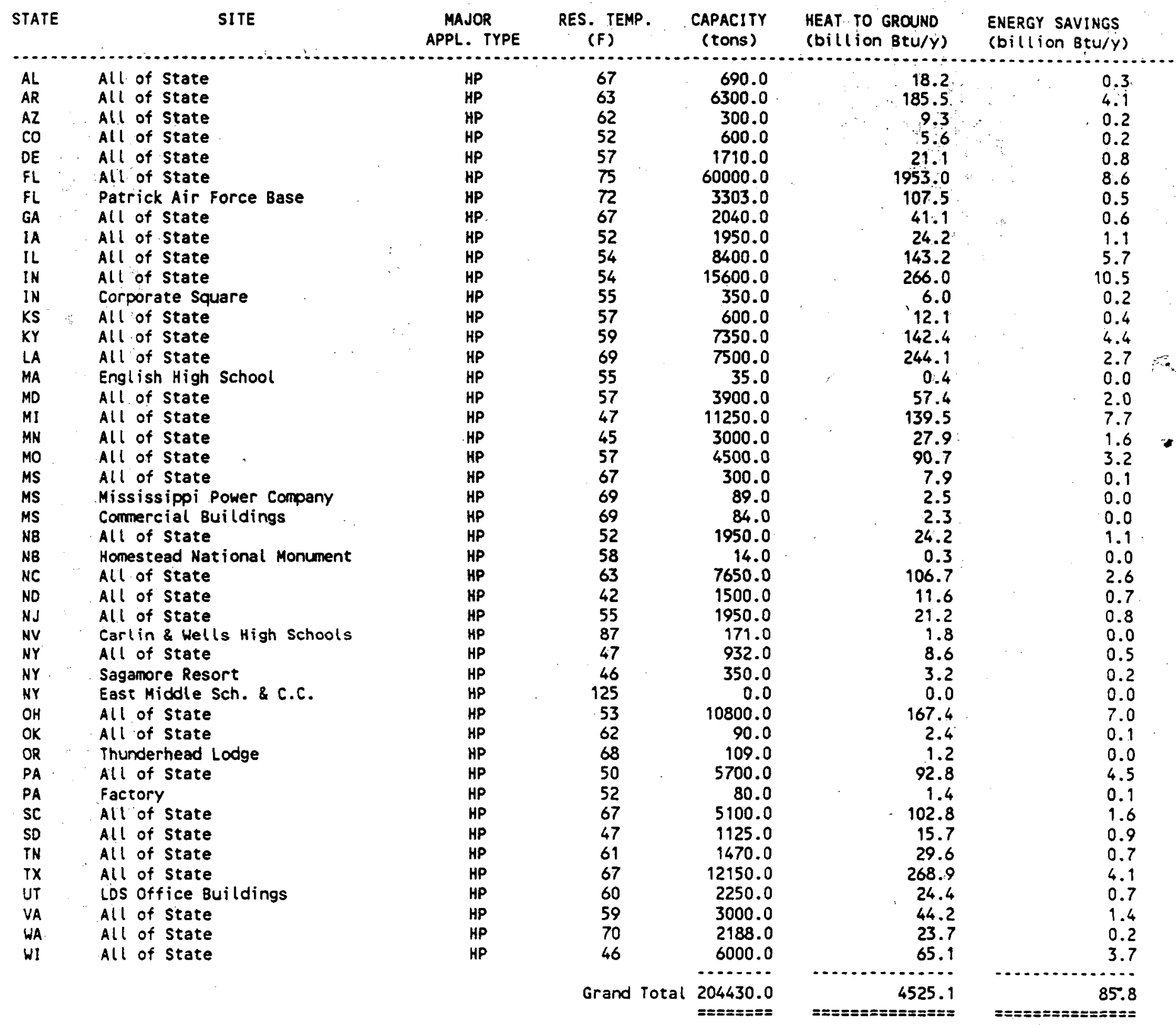


REFERENCES

Aiken, H. Personal Communication. Architect, Atlanta, GA; 1988 .

Allen, E. Preliminary Inventory of Western U.S. Cities with Proximate Hydrothermal Potential. VBB Allen, Salem, OR.

Anderson, K. Personal Communication. Maintenance Manager of Pah Tempe, UT; 1988.

Aschoff, G. Personal Communication. Manager of Vichy Springs. Ukiah, CA; 1988.

ASHRAE. American Society of Heating, Refrigeration and Air Conditioning Engineers (1985) Fundamentals. New York, N.Y., p. $28.7 ; 1985$.

Austin Park. Personal Communication. Recreation Supervisor, Austin, TX; 1988 .

Beck, R. Personal Communication. Manager of Pegasus Gold Corporation, Florida Canyon Mine, NV.; 1988 .

BEG. Personal Communication. Texas Bureau of Economic Geology, Austin, TX; 1988.

Bennette, J. Personal Communication. Groundwater section, Division of Environmental Management, Raleigh, NC; 1988.

Blacket, R. Personal Communication. Utah Geological and Mineral Survey. Salt Lake City, UT; 1988.

Bloomquist, R.G. Geothermal Energy in Washington--Site Data Base and Development Status, report to USDOE. 1979; available from Geo-Heat center and NTIS.

Bloomquist, G. Personal Communication. Washington state Energy Office. Olympia, WA; 1988.

Breckenridge, Rand. B.S. Hinkley. Thermal Springs of Wyoming. Bulletin 60, Wyoming Geological Survey, Laramie, WY; 1978 .

Brown, Keith E. Geothermal Energy in Montana--site Data Base and Development Status, report to USDOE. 1979; available from Geo-Heat center and NTIS.

Butterfield, F. Personal Communications. Pasa Robles, CA; 1988 . 
CDMG. California Division of Mines and Geology open File Report 82-4. Sacramento, CA; 1982.

Chitwood, R. Personal Communication. Mt. Shasta, CA; 1988 .

Crenshaw, H. Personal Communication. Hot Springs National Park, Hot Springs, AR; 1988.

Culver, G. Personal Communication. Geo-Heat Center, Klamath Falls, OR; 1988 .

Davenport, D. Personal Communications. Cal Pines, CA; 1988 .

Degaramo, L. Personal Communication. Ft. Bidwell Indian Reservation Tribal office, Ft. Bidwell, CA; 1988.

Dellinger, M. Geothermal Energy in the Northwest: site Specific Development Analysis. Report to USDOE. Geo-Heat Center, Klamath Falls; OR; 1982.

Dellinger, M. Personal Communication. Lakeport, CA; 1988 .

Deluca, I. Personal Communication. Pacific Power. Yakima, WA; 1988.

Eastmond, M. Personal Communication. Owner, Saratoga H.S., UT; 1988 .

Easton, M. Personal Communication. DeMaris H.S., WY: 1988

Eichman, J. Personal Communication. Supt. Hot Creek Hatchery, Mammoth Lakes, CA; 1988.

Ellis, D. Personal Communication. President of the International Ground Source Heat Pump Association and Vice Presidennt of Marketing. Waterfurnace International, Inc., Fort Wayne, IN; 1988.

Engler, B. Personal Communication. Niland, CA; 1988.

Eversoll, D. Personal Communication. Nebraska Geological Survey, University of Nebraska, Lincoln, NB; 1988.

Fischer, K. Personal Communication. San Bernardino Water Dept., San Bernardino, CA; 1988.

Fitzsimmons, K. Personal Communication. Environmental Research Laboratory, University of Arizona, Tucson, AZ; 1988 . 
Frazee, J. Personal Communication. Chief Hydrologist for st. Johns Water Management District, orlando, FL; 1988.

Fuggit, T. Personal Communication. Water Resources Department, Little Rock, AR; 1988.

GHC Bulletin. "The Geothermal Pipeline." Geo-Heat center, Bulletin Vol. 10, No. 1; 1987.

Gannett, M. Personal Communication. oregon Department of Water Resources. Salem, OR; 1988.

Gonsorcik, P. Personal Communication. Chamberlain, SD; 1988 .

Goodman, J. Personal Communication. South Dakota Department of Water Resoures, Pierre, SD; 1988.

Gordon, S. Personal Communication. Kennedy, Jencks \& Chilton Engineers, Reno, NV; 1988.

Grajcier, D. Personal Communications. Mecca, CA; 1988.

Green, K. Personal Communications. Butte, MT; 1988.

Hartford, S. Personal Communications. Oregon Department of Water Resources, Salem, OR; 1988.

Heasler, H. Personal Communication. Wyoming Commercialization Office, Laramie, WY; 1988.

Holmgren, S. Personal Communication. Manager Belmont Springs, UT; 1988 .

Huttrer, J. Personal Communication. Geothermal Management Company, Evergreen, CO; 1988.

JNFH. Personal Communication. Jackson National Fish Hatchery, Jackson, WY; 1988.

James, R. Geothermal Energy in Wyoming-site Data Base and Development Status, report to USDOE. 1979; available from Geo-Heat center and NTIS.

Justus, D. Geothermal Resources in Oregon--Site Data Base and Development Status, report to USDOE. 1979; available from Geo-Heat center and NTIS.

Karrat, c. Personal Communication. Midway, UT; 1988.

Kaysing, B. Great Hot Springs of the West. Capra Press. 
Santa Barbara, CA; 1984 .

Kehl, M. Personal Communication. Troy-Hygro Greenhouses. Sandy, UT; 1988.

Keller, K. Personal Communication. Lava Hot Springs, ID; 1988 .

Kenkermath, D.C., et.al. "The current Status of Geothermal Direct Use Development in the United States." $\underline{1985}$ International Symposium on Geothermal Energy, Geothermal Resources Council, Davis, CA. p. 223-236. 1985.

Krachow, B. Personal Communications. Based on report submitted to INEL, Auburn, NY; 1988.

Lewis, J. Personal Communications. System designer. Modesto, $\mathrm{CA}$; 1988 .

Iienau, P. "Geothermal District Heating Project." District Heating Vol. 70 , No. $1 \& 2$, IDHA, Washington, D.C.; 1984.

Lienau, P. "Status of Direct Heat Project's in Western states." GRC Transactions"Vol. 10, Davis, CA; 1986.

Loam, J. Hot Springs and Pools of the Northwest. Capra Press, Santa Barbara, CA; 1980.

Loam, J. and G. Sohler. Hot springs and Hot Pools of the Southwest. Wilderness Press, Berkeley, CA; 1985.

Lund, J. "Geothermal De-icing of Highway Pavement." Geo-Heat Center Bulletin Vol 1, No 2; 1976.

Lund, J. "USA Experience in Direct Heat Use." International Workshop on Development and Utilization of Small Geothermal Resources, Pisa, Italy; 1987.

Lunis, B.C. Geothermal Direct Use Program Opportunity Notice projects Lessons Learned. Idaho National Engineering Laboratory, 1986.

Lunis, B.C. (1988). Geothermal Direct Use Projects in the United State - Status and Trends. INEL paper for Jigastock 88, Idaho Falls; ID.

Luza, K. Personal communication. Geological Survey, Oklahoma City, OK; 1988.

McAfee, B. Personal Communication. English High School, 
Boston, MA; 1988.

MCClain, D. Geothermal Energy in Idaho--Site Data Base and Development Status, report to USDOE. 1979; available from Geo-Heat center and NTIS.

Mccray, K. Personal Communication. National Water well Association, Dublin, $\mathrm{OH}$; 1988 .

McGraw, K. Heat Pump Applications. Oregon Air Representatives, Inc., Portland, OR; 1984.

McKay, F. Personal Communication. McKay Well Drilling, Reno, NV; 1988.

Mclemone, B. Personal Communication. State Geologist, Atlanta, GA; 1988 .

McMurray, K. Personal Communications. Maintenance, Homestead National Monument, Beatice, NB; 1988.

Makey, D. Personal Communication. Owner, Crystal H.S., UT; 1988 .

Markle, D. Geothermal Energy in Alaska--Site Data Base and Development Status, report to USDOE. 1979; available from Geo-Heat center and NTIS.

Martinez, J. Personal Communications. Pagosa Springs, Co; 1988 .

Minto, C. Personal Communication. Gerlach Postmistress, Gerlack, NV; 1988.

Muffler, L.J.P., (editor). Assessment of Geothermal Resources of the United States - 1978. US Geological Survey Circular 790, Reston, VA; 1979.

NocS. Renewable Energy in Nevada. Nevada office of Community Services, Carson City, NV; 1987.

NWWA. Ground Water Heat Pumps. National Water Well Association, Dublin, OH; 1988

Norton, D. Personal Communicaton. PEC Corp., Witchita, KS ; 1988 .

Nydal, J. Evaluation of Earth Heated Bridge Deck. University of Wyoming Report, Laramie, WY; 1984.

Pasquelleti, M. Personal Communication. Arizona State 
University, Mesa, AZ; 1988

Prem, B. Personal Communication. Maintenance Supervisor, Glenwood Hot Springs; CO; 1988.

Rafferty, K. Economic Analysis of Indian Valley Hospital Geothermal Heat Pump system. Geo-Heat Center Report, Klamath Falls, OR; 1988 .

Rafferty, K. Greenhouse Heating System. Geo-Heat Center Report; 1981.

Rafferty, K. Heating Facilities for Moana Municipal Pool, Geo-Heat Center Report; 1980.

Rafferty, K. Personal Communication. OIT Geo-Heat Center, Klamath Falls, OR; 1988.

Ramsey, M. Personal Communication. Maintenance Supervisor, Cotulla, TX; 1988 .

Reed, M. (editor). Assessment of Low Temperature Geothermal Resources of the United States - 1982. U.S. Geological Survey Circular 892, Reston, VA; 1982.

Rold, J. Personal Communication. Colorado Geological Survey, Denver, Co; 1988.

Rutten, P. Personal Communication. Vale, OR; 1987.

Sammel, E. "Hydrogeologic Appraisal of the Klamath Falls Geothermal Area, Oregon." Geological survey Professional Paper 1044-G; 1980.

Schultz, B. Personal Communication. Driller, Greenville, MS ; 1988 .

Sonderegger. Geothermal Resources of Montana. Map produced by NOȦ, Washington, D.C.; 1981.

Street, L. "In Southern Idaho - Greenhouses Use Geothermal Heat to Cut Energy Bills." Geo-Heat Center Bulletin, Vol. 9, No. 2. 1985.

Trexler, D. Personal Communication. Divison of Earth Sciences, UNLV, Reno, NV; 1988.

Tringle, B. Personal Communication. Waunite Hot Springs Ranch, CO; 1988.

Vance, D. Personal Communication. Red Bluff, CA; 1988. 
Waldick, R. Personal Communication. Homestead Resort, Hot Springs, AR; 1988 .

Witcher, J. Personal Communication. University of New Mexico, Las Cruces, NM; 1988.

Woodruff, J. Personal Communication. Hawaii Natural Energy Institute, University of Hawaii at Monoa, Honolulu, HI; 1988 . 
APPENDIX

DESCRIPTIONS OF DIRECT USE SITES 
SITE: Chena Hot Springs

LOCATION: $70 \mathrm{mi}$ NE Fairbanks

APPLICATION TYPE: S, G, P

RESOURCE TEMP.: $135 \mathrm{~F}$

MAX. FLOW: $222 \mathrm{gpm}$

THERMAL CAPACITY: $\quad 3.3 \mathrm{mill}$ ion $\mathrm{Btu} / \mathrm{h}$

ANNUAL ENERGY: $\quad 15.5$ billion 8 tu/Y

DESCRIPTION: Space heating a lodge and greenhouse and for swimming and therapeutic pools.

REFERENCE: Markle, 1979

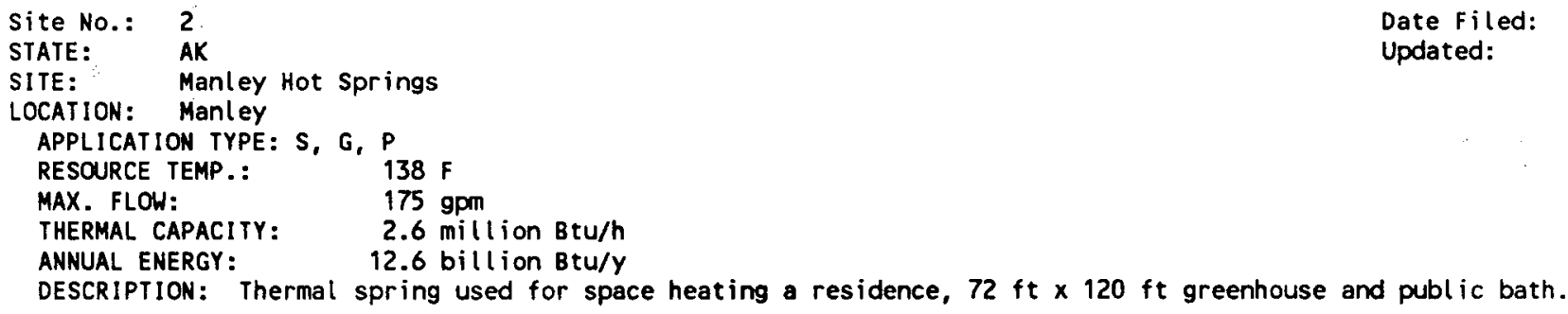


REFERENCE: Markle, 1979

Site No.: $\quad 7$
STATE: $\quad$ AK
SITE: Goddard
LOCATION: 15 mi S of Sitka
APPLICATION TYPE: P $\quad 153 \mathrm{f}$
RESOURCE TEMP.: $\quad 13 \mathrm{gPm}$
MAX. FLOW:
THERMAL CAPACITY: $\quad 0.1 \mathrm{million}$ Btu/h
ANNUAL ENERGY: $\quad 0.7$ billion Btu/y
DESCRIPTION: Thermal spring used for bath house.

REFERENCE: Markle, 1979

Site No.: 8
STATE: AK
SITE: Baranof
LOCATION: $20 \mathrm{mi}$ E of sitka
APPLICATION.TYPE: S, P
RESOURCE TEMP.: $\quad 151 \mathrm{~F}$
MAX. FLOW: $\quad 100 \mathrm{gpm}$
THERMAL CAPACITY: $\quad 0.1 \mathrm{million}$ Btu/h
ANNUAL ENERGY:
DESCRIPTION: Thermal spring used to space heat a residence and small bath house.

REFERENCE: Markle, 1979

\begin{tabular}{|c|c|}
\hline $\begin{array}{ll}\text { Site HO.: } & 9 \\
\text { STATE: } & \text { AK } \\
\text { SITE: } & \text { Ophir Creek } \\
\text { LOCATION: SW Region } \\
\text { APPLICATION TYPE: S } \\
\text { RESOURCE TEMP.: } \\
\text { MAX. FLOW: } \\
\text { THERMAL CAPACITY: } \\
\text { ANNUAL ENERGY: }\end{array}$ & $\begin{array}{l}145 \mathrm{~F} \\
225 \mathrm{gpm} \\
0.1 \mathrm{mill} \\
0.2 \text { billion Btu/h } \mathrm{Btu} / \mathrm{y}\end{array}$ \\
\hline
\end{tabular}

REFERENCE: Markle, 1979

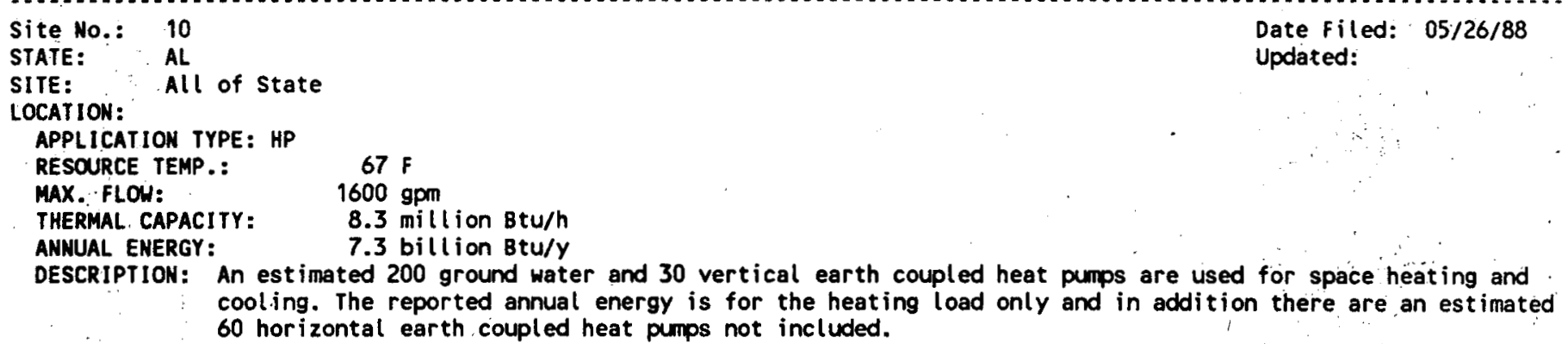


LOCATION: Mainly Eastern Portion APPLICATION TYPE: HP RESOURCE TEMP.

MAX. FLOW:

THERMAL CAPACITY:

DESCRIPTION: An estimated 3000 ground water heat pump wells are used for space heating and cooling. These wells represent 20 percent ground water, 50 percent vertical earth coupled, and the remaining are horizontal earth coupled. The reported annual energy is only for the heating load.

REFERENCE: Fuggit, 1988; Ellis, 1988

Site No.: 12

STATE: AR

SITE: $\quad$ Hot Springs National Park

LOCATION: HOt SPrings

APPLICATION TYPE: S, P

RESOURCE TEMP.: $139 \mathrm{~F}$

MAX. FLOW: $250 \mathrm{gpm}$

THERMAL CAPACITY: $\quad 1.2 \mathrm{mill}$ ion Btu/h

ANNUAL ENERGY: $\quad 6.9$ billion Btu/Y

DESCRIPIION: Space and water heating of administrative buildings, bathhouse, and one hotel.

REFERENCE: Crenshaw, 1988

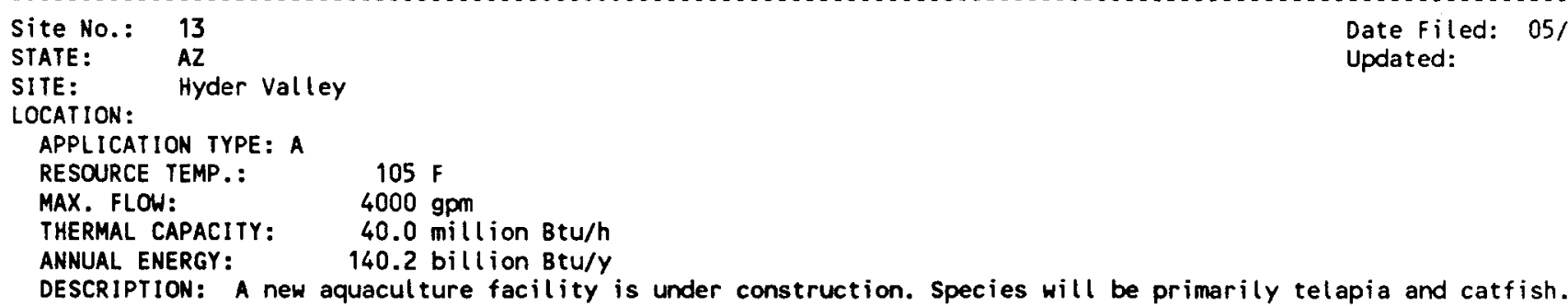

REFERENCE: Fitzsimons, 1988

Site No.: $\quad 14$
STATE: $\quad$ AZ
SITE: $\quad$ Safford
LOCATION: Safford
APPLICATION TYPE: A
RESOURCE TEMP.: $\quad 105 \mathrm{~F}$
MAX. FLOW:
THERMAL CAPACITY: $\quad 1000 \mathrm{gPm}$
ANNUAL ENERGY: $\quad 35.0 \mathrm{milli}$ billion Btu/h
DESCRIPTION: An aquaculture facility growing catfish. Utilizes one warm well (105 F; approx. $1,000 \mathrm{gpm})$ and one cool
$\quad$ well $(1,000 \mathrm{gPm})$

REFERENCE : Fitzs immons, 1988

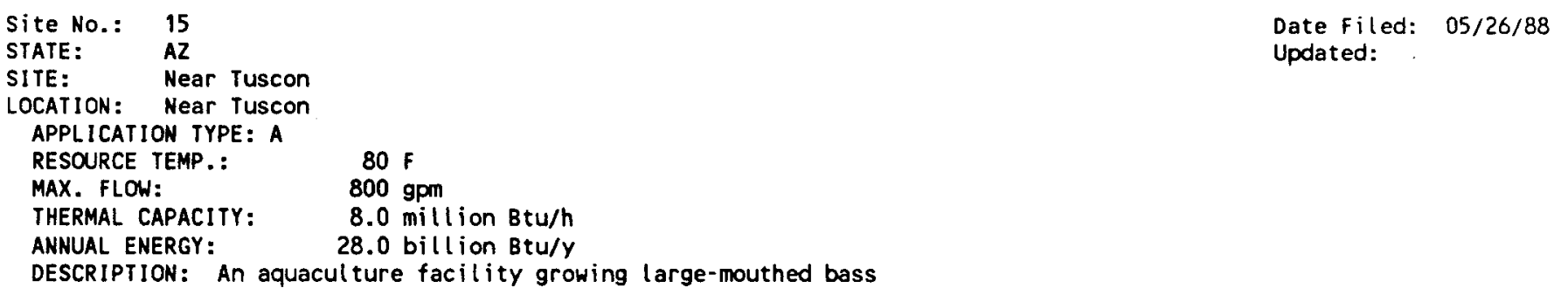


APPLICATION TYPE: A

RESOURCE TEMP.: $105 \mathrm{~F}$

MAX. FLOW: $\quad 850 \mathrm{gPm}$

THERMAL CAPACITY: $\quad 7.0 \mathrm{mill}$ ion Btu/h

ANNUAL ENERGY: $\quad 24.5$ billion Btu/Y

DESCRIPTION: In the Hyder Valley six aquaculture facilities use 400-850 gpm each of 80-105 $\mathrm{F}$ geothermal water. Species grown are telapia, catfish and large mouth bass. Note: Arnual use estimate based on a 0.4 utilization factor $85 \mathrm{~F}$ pond temp. Use is probably conservative.

REFERENCE: Fitzsimmons, 1988

Site NO.: 17
STATE: $\quad$ AZ
SITE: $\quad$ All of State
LOCATION:
APPLICATION TYPE: HP
RESOURCE TEMP.:
MAX. FLOW:

REFERENCE : Ellis, 1988

Site NO.: 18
STATE:
SITE: $\quad$ Castle Hot Springs
LOCATION: Near Wickenberg
APPLICATION TYPE: P
RESOURCE TEMP.: $\quad 131 \mathrm{~F}$
MAX. FLOW:
THERMAL CAPACITY: $\quad 340 \mathrm{gpm}$
ANNUAL ENERGY: $\quad 0.5 \mathrm{mill}$ U
DESCRIPTION: An Etu/h
used by the Vanderbilts, Kennedys, etc., then donated to Arizona State University for a conference
retreat. Recently sold to a private developer.

REFERENCE: Pasquelleti, 1988

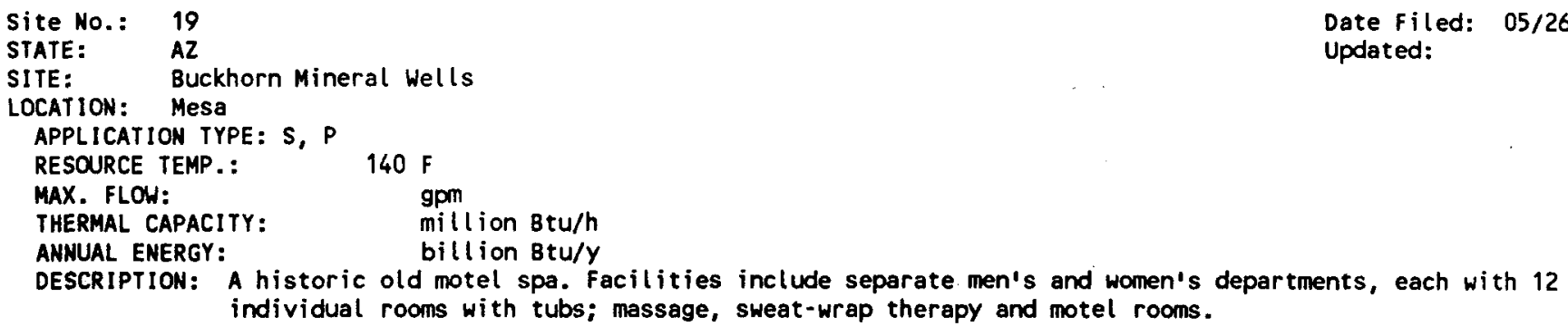




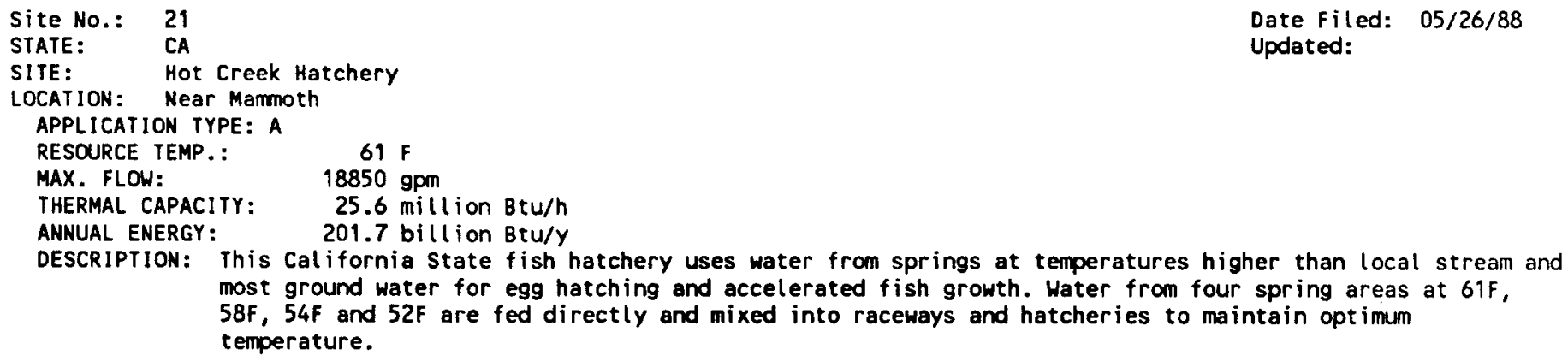

REFERENCE: Eichman, 1982

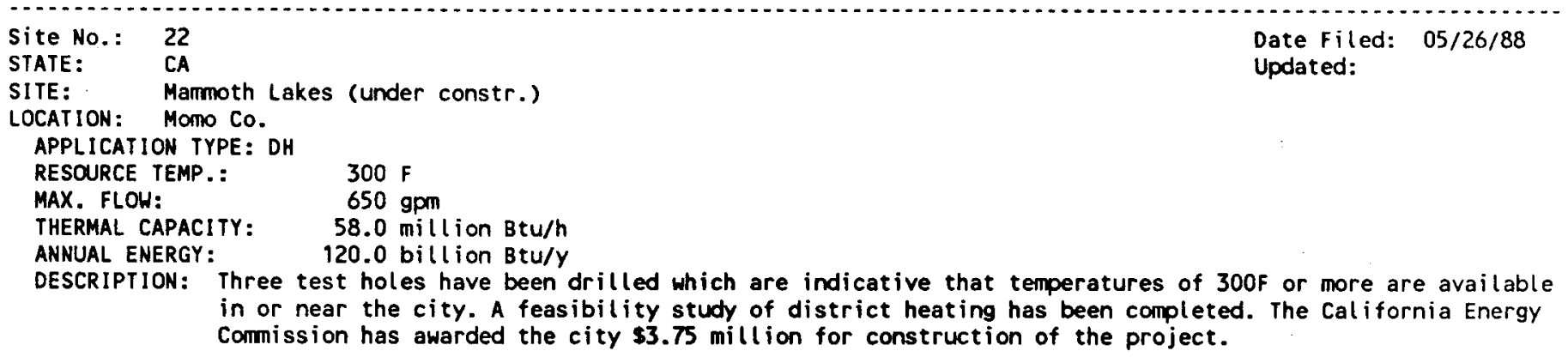

REFERENCE: Culver, 1988

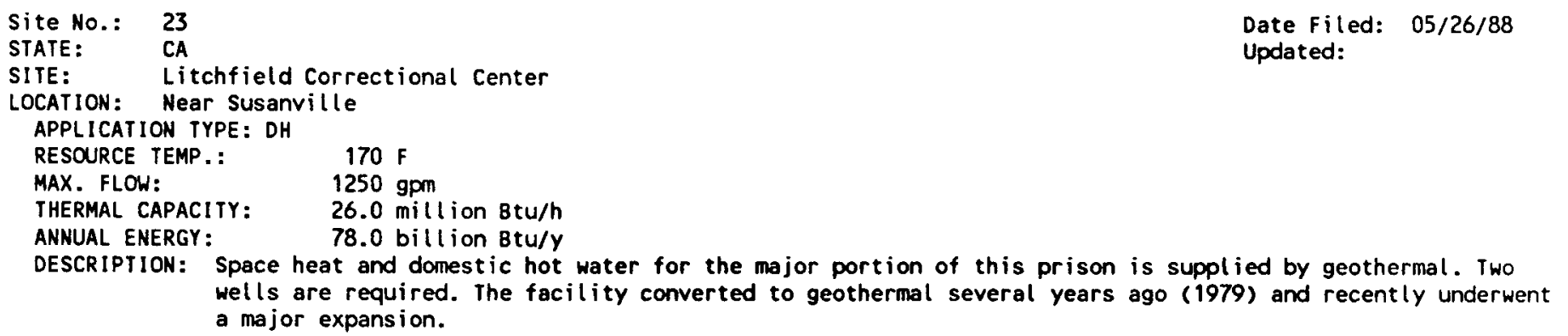

REFERENCE: Culver, 1988

Site No.: 24
STATE: $\quad$ CA
SITE: $\quad$ Paso Robles Fish Farm
LOCATION: SanLUis Obispo Co
APPLICATION TYPE: A $\quad 104 \mathrm{~F}$
RESOURCE TEMP.: $\quad 1000 \mathrm{gpm}$
MAX. FLOW:
THERMAL CAPACITY: $\quad 10.0 \mathrm{mill}$ ion Btu/h
ANNUAL ENERGY: $\quad 70.0$ billion Btu/y
DESCRIPTION: This aquaculture project operated for a nmber of years, then closed due to problems after an owner's
death. It has recently reopened.

REFERENCE: Butterfield, 1988

Site No.: 25
STATE: $\quad$ CA
SITE: $\quad$ Pacific Aqua Farms
LOCATION: Near Niland
APPLICATION TYPE: A $\quad 140 \mathrm{~F}$
RESOURCE TEMP.: $\quad 650 \mathrm{gpm}$
MAX. FLOW: $\quad 17.9 \mathrm{million} \mathrm{Btu/h}$
THERMAL CAPACITY: $\quad 62.6$ billion Btu/y
ANNUAL ENERGY: $\quad$ Updated:
DESCRIPTION: Water from a geothermal well is utilized to maintain temperature for growing telapia in ponds at $85 \mathrm{~F}$ -
utilization factor 0.4 . Only source of water. Only problem is cooling it in summer. Has been in
operation 6 years.


SITE: $\quad$ Bridgeport (under constr.)

LOCATION: Bridgeport, Mono Co.

APPLICATION TYPE: DH

RESOURCE TEMP.:

MAX. FLOW:

$170 \mathrm{~F}$

THERMAL CAPACITY:

$800 \mathrm{gpm}$

ANNUAL ENERGY:

$13.0 \mathrm{mill}$ ion Btu/h

27.0 billion Btu/y

DESCRIPTION: Production and injection wells have been drilled for a co-generation system using binary generators. All the pipeline and retrofit designs have been completed, but problems with the injection well developed. As soon as the well problem is solved, the project is expected to go forward. Funding is being provided by CEC.

REFERENCE: Culver, 1988

$\begin{array}{ll}\text { Site No.: } & 32 \\ \text { STATE: } & \text { CA } \\ \text { SITE: } & \text { Vichy Hot Springs } \\ \text { LOCATION: Near Ukiah } & \\ \text { APPLICATION TYPE: S } & \\ \text { RESOURE TEMP.: } & 90 \mathrm{~F} \\ \text { MAX. FLOW: } & 65 \mathrm{gpm} \\ \text { THERMAL CAPACITY: } & 0.1 \mathrm{mill} \text { ion Btu/h } \\ \text { ANNUAL ENERGY: } & 26.4 \mathrm{billion} \text { Btu/y }\end{array}$

lion Btu/Y

DESCRIPTION: Capacity: 26,380. The spring is used primarily as a source for bottled mineral water. However, there is a kindergarden/day school set up in one of the old resort hotel buildings that is geothermally heated. At one time this was a well-known hot springs resort with several pools, hotel, guest houses, large dance hall, etc.

REFERENCE: Culver 88; Gilbert Aschoff 88

Site No.: 33
STATE:
SITE:

REFERENCE: CDMG, 1982

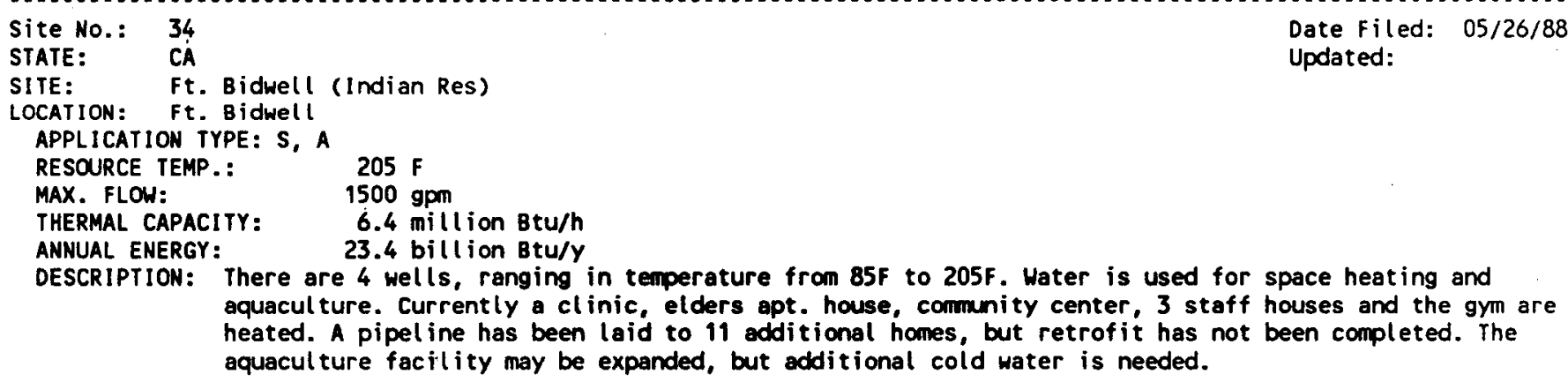

REFERENCE: Degarmo, 1988

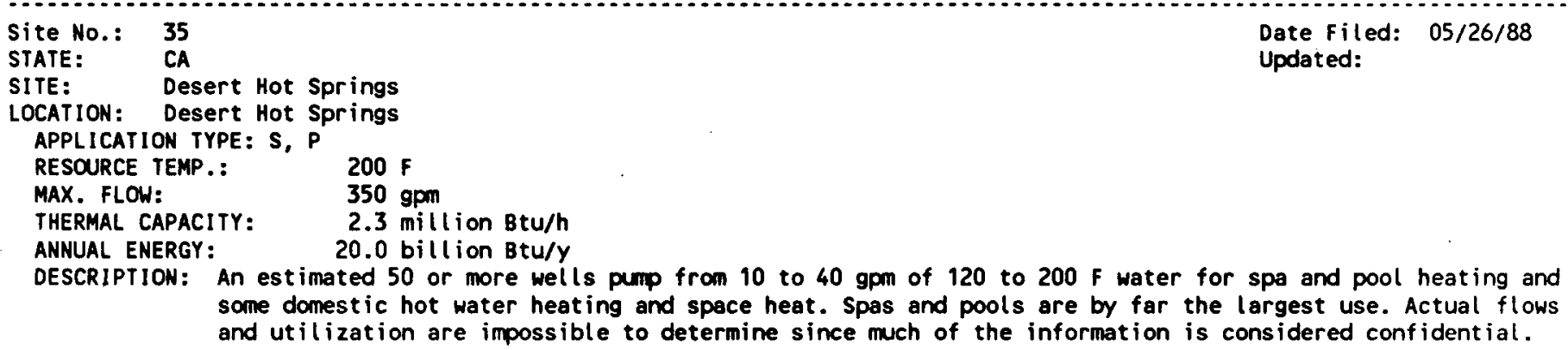


SITE: $\quad$ SUsanville District Heating

LOCATION: SUSANVIlle, CA

APPLICATION TYPE: DH

RESOURCE TEMP.: $\quad 170 \mathrm{~F}$

MAX. FLOW: $\quad 750 \mathrm{gPm}$

THERMAL CAPACITY: $\quad 8.0 \mathrm{mill}$ ion Btu/h

ANNUAL ENERGY: $\quad 18.0$ billion Btu/y

DESCRIPIION: A district heating system supplies heat to schools, public buildings, swimming pool, county and city shops, residences, etc. The system pumps $170 \mathrm{~F}$ geothermal water from one well to the applications where heat exchangers are installed with closed loop building systems. Geothermal discharge is about $130 \mathrm{~F}$ partially injected and partial to surface discharge. A new injection well will be drilled April, 1988, REFERENCE: Culver, 1988 to take all the discharge.

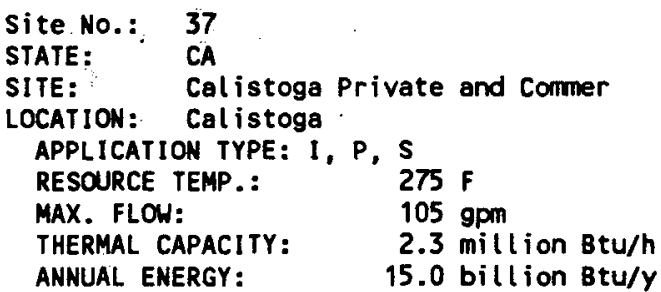

DESCRIPTION: At least 34 wells with temperatures over 100f are utilized by mineral water bottlers, spas, pools; mud baths and space heating of both comercial space and private residences. There is no accurate assessment of use, but the yearly flow is estimated to be well over 55 million

REFERENCE: CEC-187; CDMG-182; Culver-188

Site No.: 38
STATE: $\quad$ CA
SITE: $\quad$ Nakashima Nurseries
LOCATION: COachella
APPLICATION TYPE: G $\quad 120 \mathrm{~F}$
RESOURCE TEMP.: $\quad 2000 \mathrm{gpm}$
MAX. FLOW: $\quad 15.0 \mathrm{million}$ Btu/h
THERMAL CAPACITY: $\quad 13.1$ billion Btu/y
ANNUAL ENERGY: $\quad$ Updated:
DESCRIPTION: These greenhouses are one of the largest cut roses producers in California.

REFERENCE: Lienau, 1986

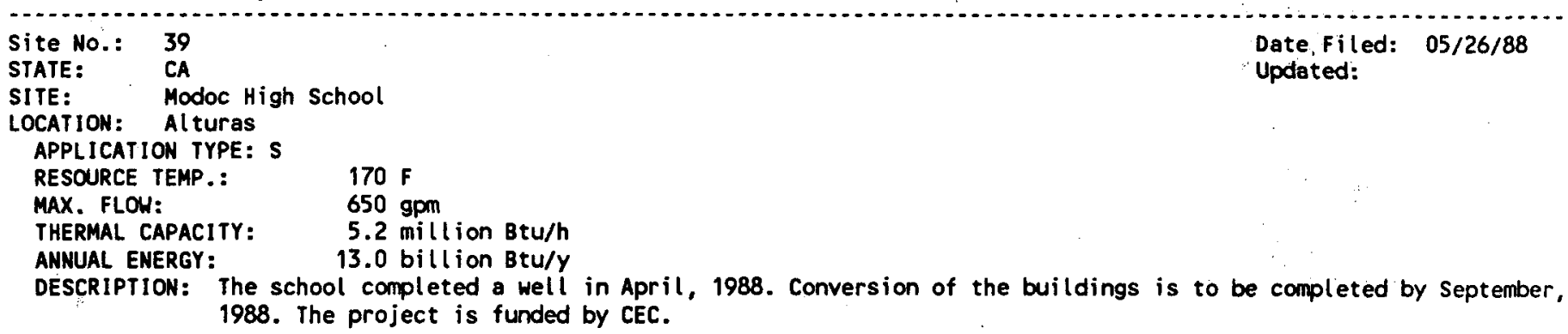

REFERENCE: Culver, 1988

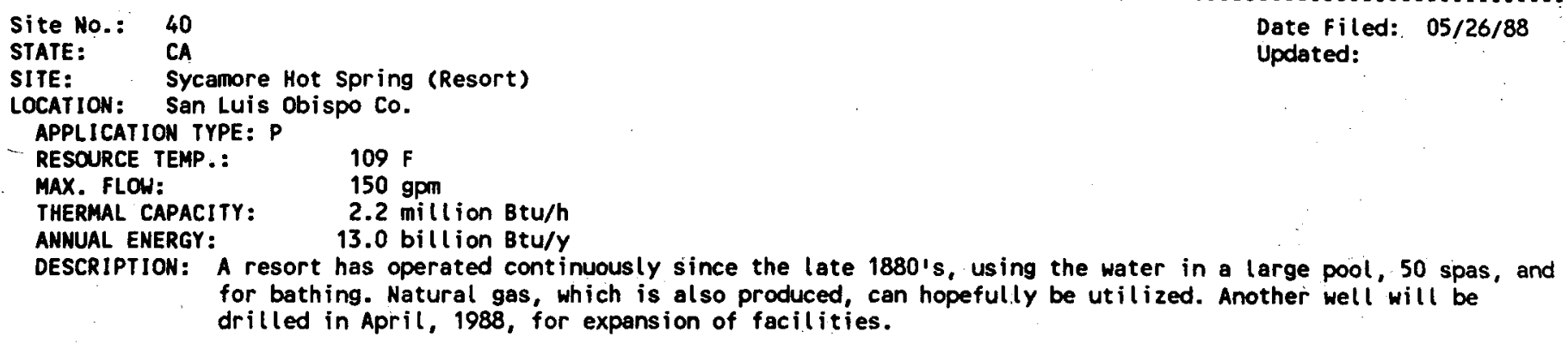


SITE: $\quad$ Aqua Caliente Springs Resort

LOCATION: SONOMa CO.

APPLICATION TYPE: P

-RESOURCE TEMP.: $\quad 97 \mathrm{~F}$

MAX. FLOW: $\quad 70 \mathrm{gpm}$

THERMAL CAPACITY: $\quad 1.4 \mathrm{mill}$ ion $\mathrm{Btu} / \mathrm{h}$

ANNUAL ENERGY: $\quad 12.0$ billion $B t u / Y$

DESCRIPTION: Three wells $300 \mathrm{ft}$ deep supply water for a large pool in a day use resort. The resort has operated cont inuously since May 5, 1887. Haring (1915) reported the resort had a large hotel and cottages for about 300 people, but these have been demoli shed and removed.

REFERENCE: CDMG, 1982

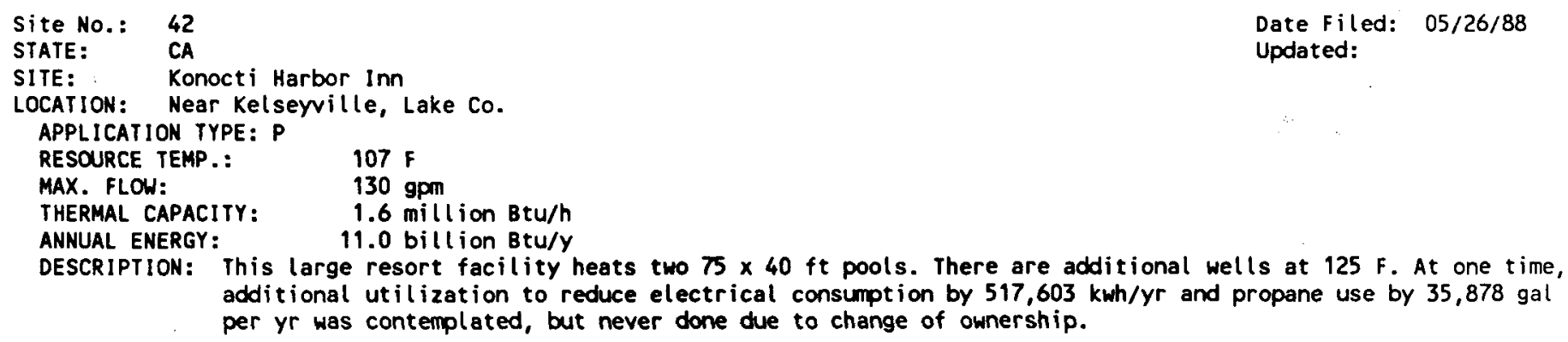
additional utilization to reduce electrical consumption by $517,603 \mathrm{kwh} / \mathrm{yr}$ and propane use by $35,878 \mathrm{gal}$ per yr was contemplated, but never done due to change of ownership.

REFERENCE: Culver, 1988

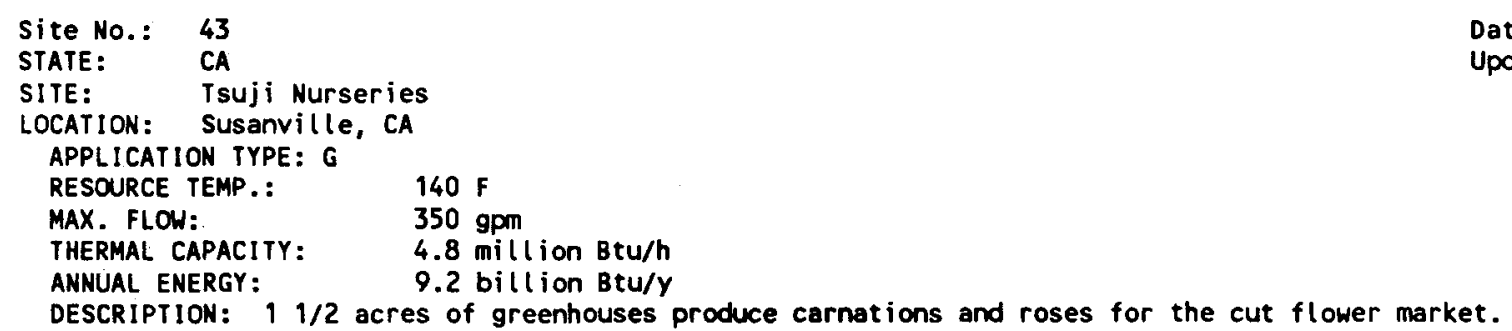

REFERENCE : Culver, 1988

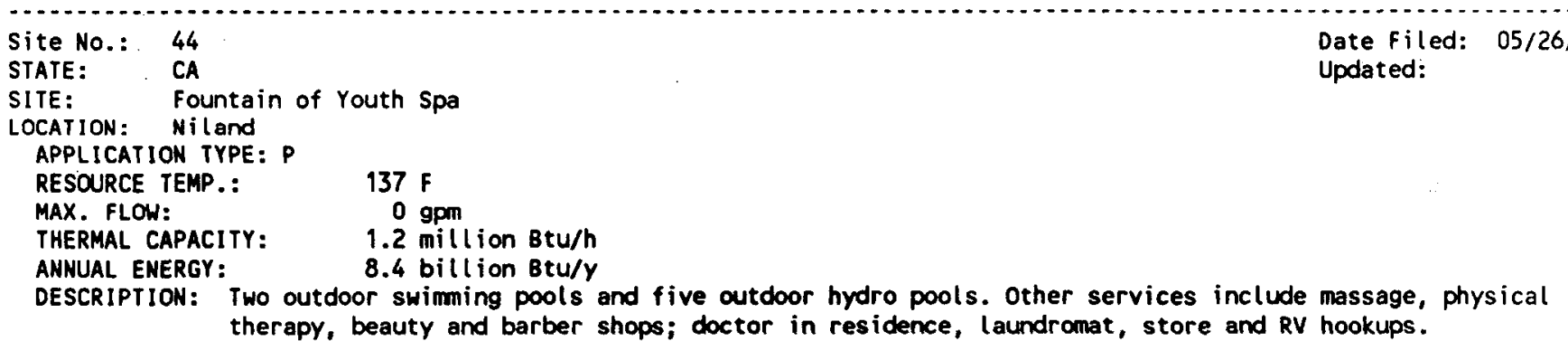

REFERENCE : Loam, 1985

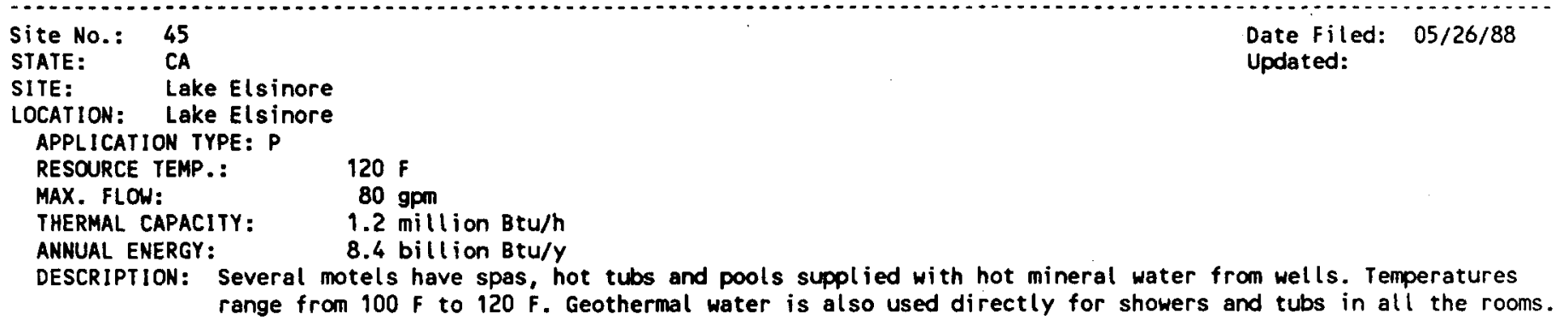


STATE: CA

SITE: Glen IVy Hot Springs (Resort)

LOCATION: Riverside CO

APPLICATION TYPE: P

RESOURCE TEMP.: $\quad 106 \mathrm{~F}$

MAX. FLOW: $\quad 165 \mathrm{gpm}$

THERMAL CAPACITY: $\quad 1.2 \mathrm{mill}$ ion Btu/h

ANNUAL ENERGY: $\quad 8.0 \mathrm{bill}$ ion $B t u / Y$

DESCRIPTION: This day resort, once a large old resort, has been in operation since about 1870. The hotel and guest houses have been converted for residential use by the owners and employees. The day use facility consists of 2 pools, a large jacuzzi, 12 mineral baths and clay pool (similar to a large mud bath) and snack bar. All the pools and tubs use mineral water.

REFERENCE: Culver, 1988

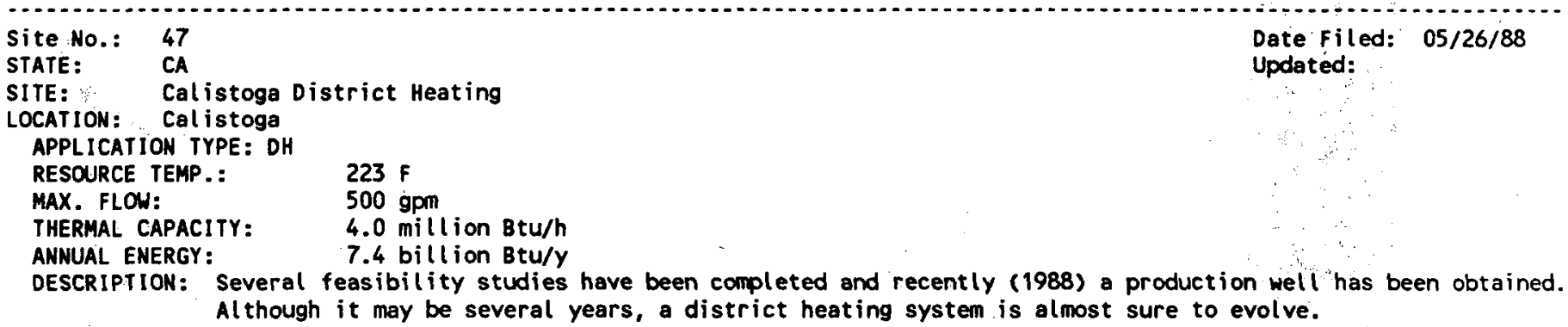

REFERENCE: Culver, 1988

Site NO.: 48
STATE: $\quad$ CA
SITE:
LOCATION: NOWn of Tecopia

REFERENCE : Loam, 1985

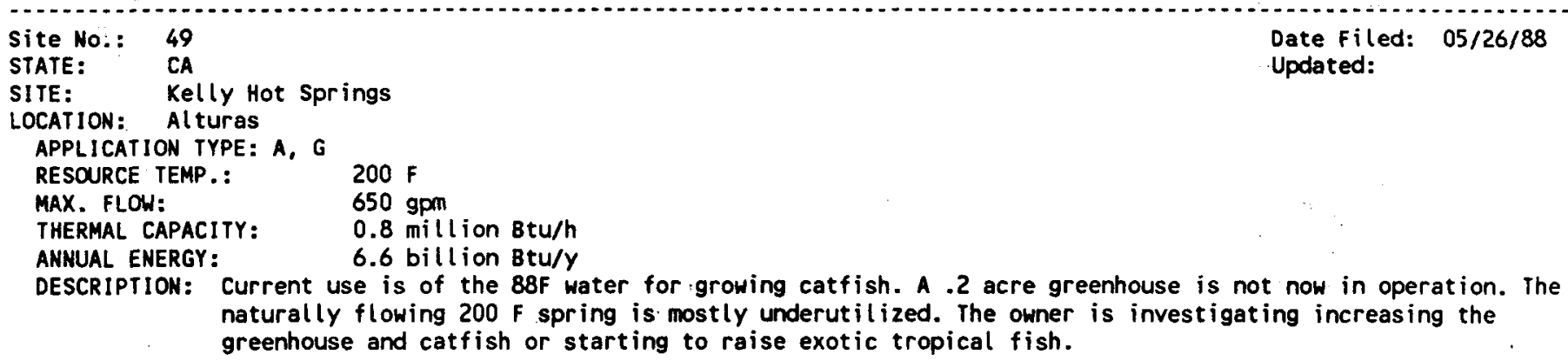

REFERENCE: Culver, 1988

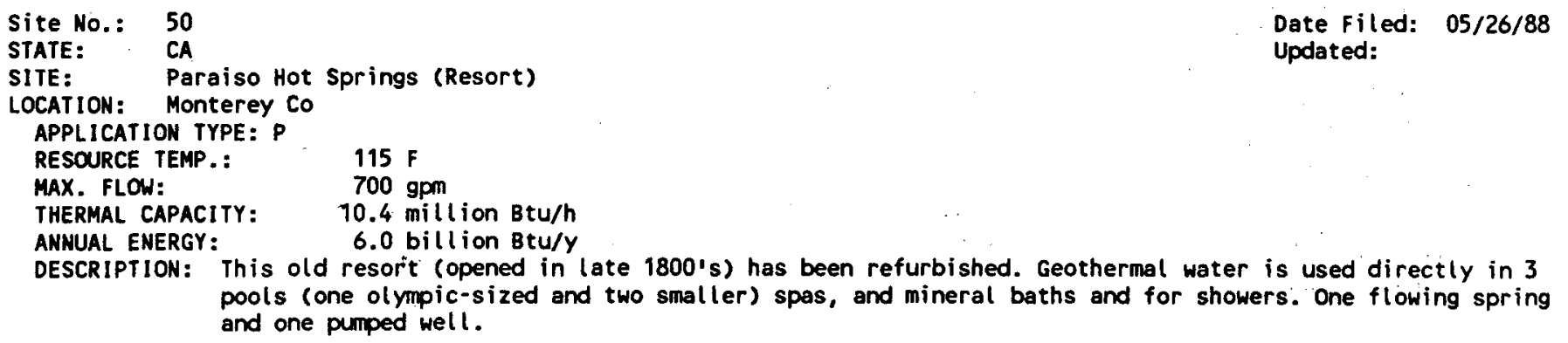


SITE: $\quad$ Tassajara Buddhist Meditation

LOCATION: Carmel Valley

APPLICATION TYPE: P

RESOURCE TEMP.: $110 \mathrm{~F}$

MAX. FLOH: 152 gpm

THERMAL CAPACITY: $\quad 0.7 \mathrm{mill}$ ion Btu/h

ANNUAL ENERGY: $\quad 5.5$ billion Btu/y

DESCRIPTION: Primarily a Buddhist Monostary with limited public accommodations. Rooms and meals with reservations; large meditation and swimming pools.

REFERENCE: Loam, 1985

Site No.: 52

STATE: $\quad$ CA

SITE: : Furnace Creek Inn

LOCATION: Death Valley

APPLICATION TYPE: P

RESOURCE TEMP.: $89 \mathrm{~F}$

MAX. FLOW: $\quad 350 \mathrm{gpm}$

THERMAL CAPACITY: $\quad 0.8 \mathrm{mill}$ ion Btu/h

ANMUAL ENERGY: $\quad 4.9$ billion Btu/y

DESCRIPTION: Two swiming pools are heated by flou through at this hotel.

REFERENCE : Loam, 1985

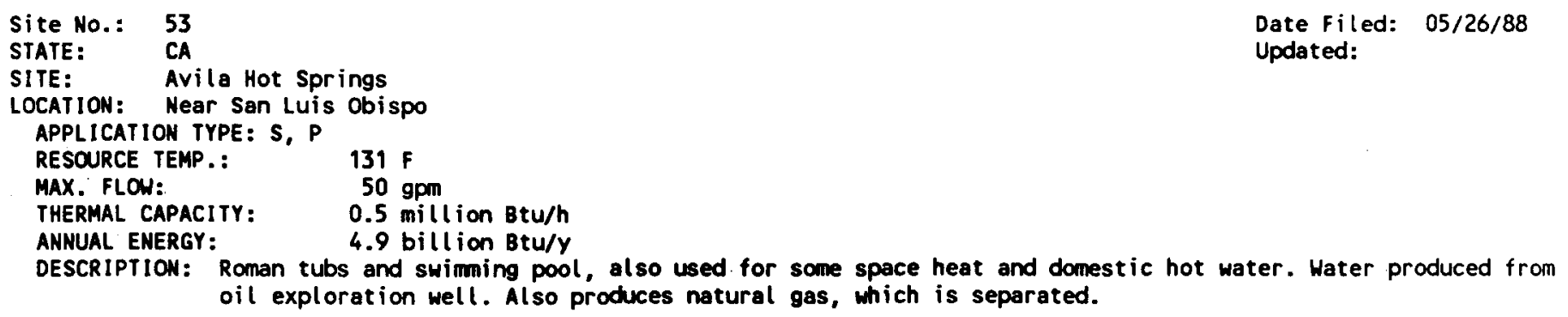

REFERENCE : HCDMG, 1982; Loam, 1985

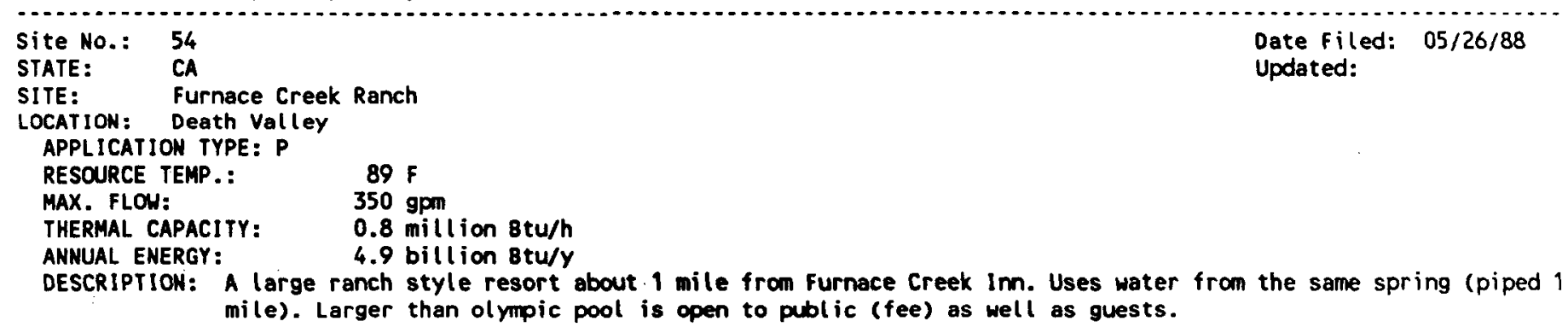

REFERENCE: Loam, 1985

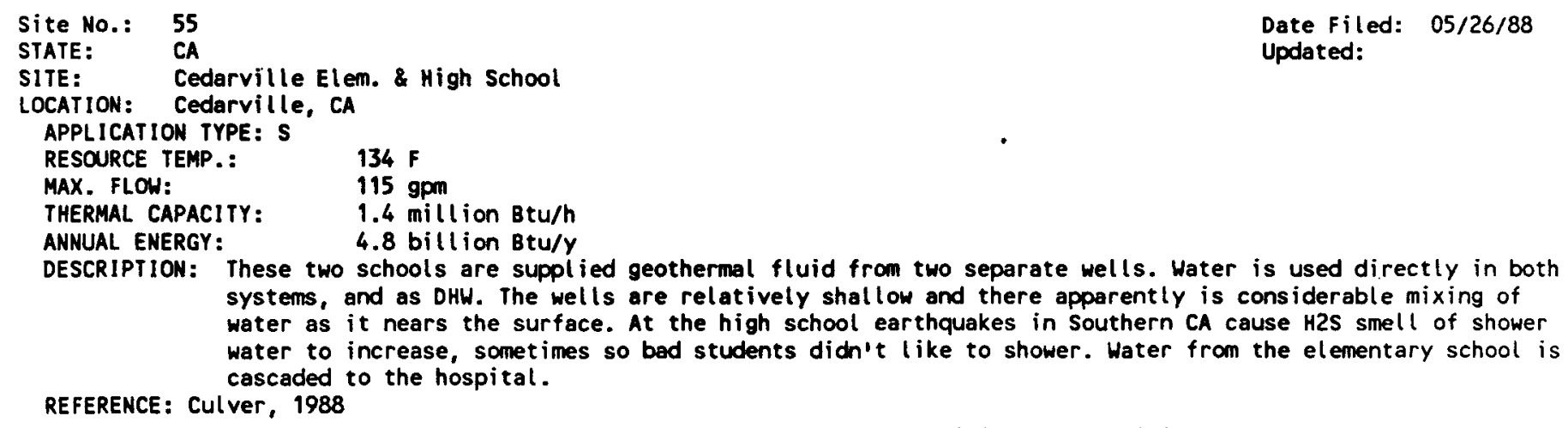


LOCATION: Alpine Co. (S of Lake Tahoe)

APPLICATION TYPE: P

RESOURCE TEMP .:

MAX. FLOW:

$147 F$

THERMAL CAPACITY: $\quad 0.6 \mathrm{mill}$ lion Btu/h

ANNUAL ENERGY: $\quad 4.7 \mathrm{bill}$ ion Btu/Y

DESCRIPTION: Large pool and soaking tubs adjoining Grover state Campground.

REFERENCE : Loam, 1985

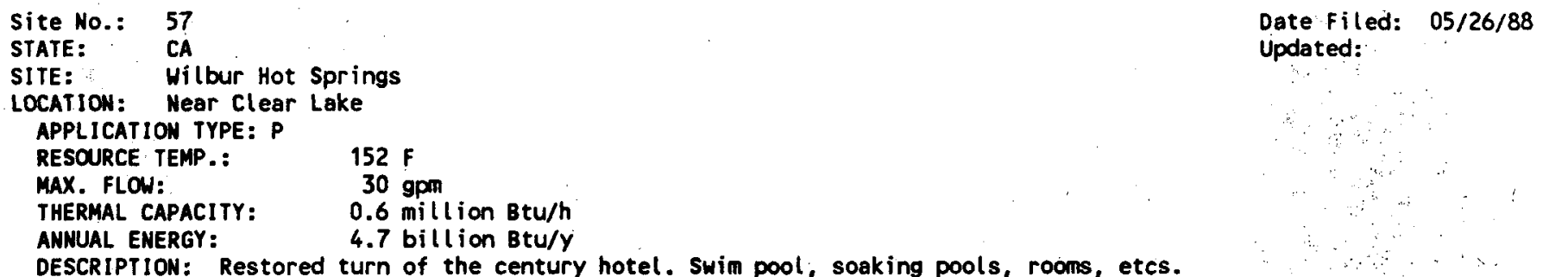

DESCRIPTION: Restored turn of the century hotel. Swim pool, soaking pools, rooms, etcs.

REFERENCE: Loam, 1985

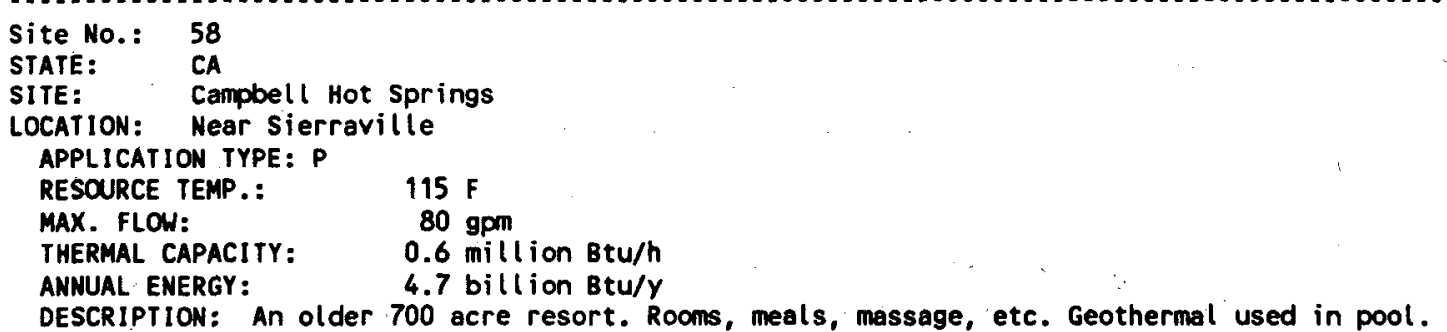

Site NO.: 59
STATE:
CATE: Whitmore Hot Springs
LOCATION: Near Bishop
APPLICATION TYPE: P
RESOURCE TEMP.: $\quad 95 \mathrm{~F}$
MAX. FLOW: $\quad 400 \mathrm{gpm}$
THERMAL CAPACITY: $\quad 0.6 \mathrm{million}$ Btu/h
ANNUAL ENERGY: $\quad 4.7$ billion Btu/y
DESCRIPTION: A large public swimming pool operated jointly by Mono County and Los Angeles City Parks \& Receation
Department.

REFERENCE: Loam, 1985; Culver, 1988

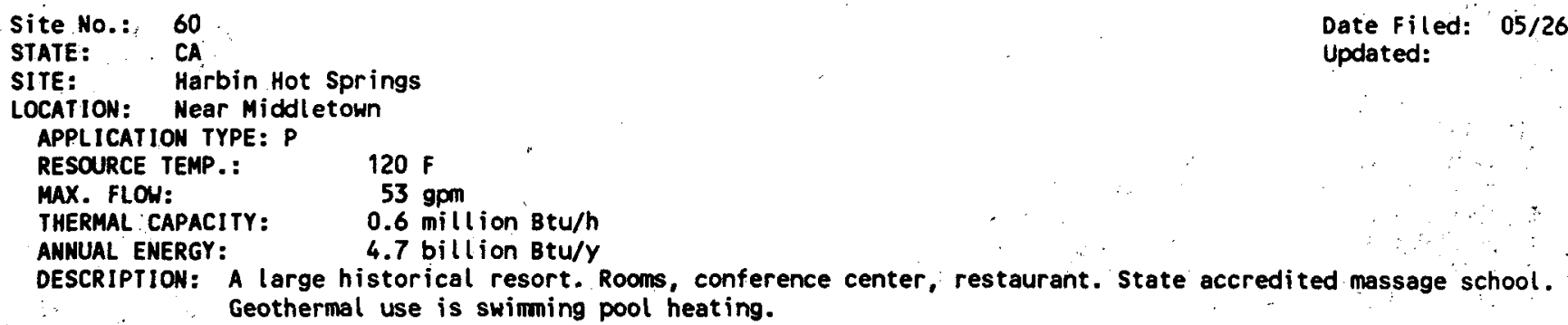


APPLICATION TYPE: S

RESOURCE TEMP.: $127 \mathrm{~F}$

MAX. FLOW: $\quad 300 \mathrm{gpm}$

THERMAL CAPACITY: $\quad 2.0 \mathrm{mill}$ ion $\mathrm{BtU} / \mathrm{h}$

ANNUAL ENERGY: $\quad 4.5$ billion $B$ tu/Y

DESCRIPTION: This complex consists of a school with large indoor pool, superintendent house and teacher's duplex. The well is $800 \mathrm{ft}$ deep and is artesian, but requires pumping for high loads in cold weather. Discharge is to surface.

REFERENCE: Culver, 1986; Chitwood, 1988

Site NO.: 62
STATE: $\quad$ CA
SITE: $\quad$ Esalen Institute
LOCATION: Near Big Sur
APPLICATION TYPE: P
RESOURCE TEMP.: $\quad 120 \mathrm{~F}$
MAX. FLOW:
THERMAL CAPACITY: $\quad 0.6 \mathrm{million}$ Btu/h
ANNUAL ENERGY: $\quad 4.4$ billion Btu/y
OESCRIPTION:
Primarilly an educational/experiential center. Also, seminars and workshops. Room and board to public is
available. Large indoor and outdoor soaking tubs available to public at night and weekends for nominal
charge.

REFERENCE : Loam, 1985

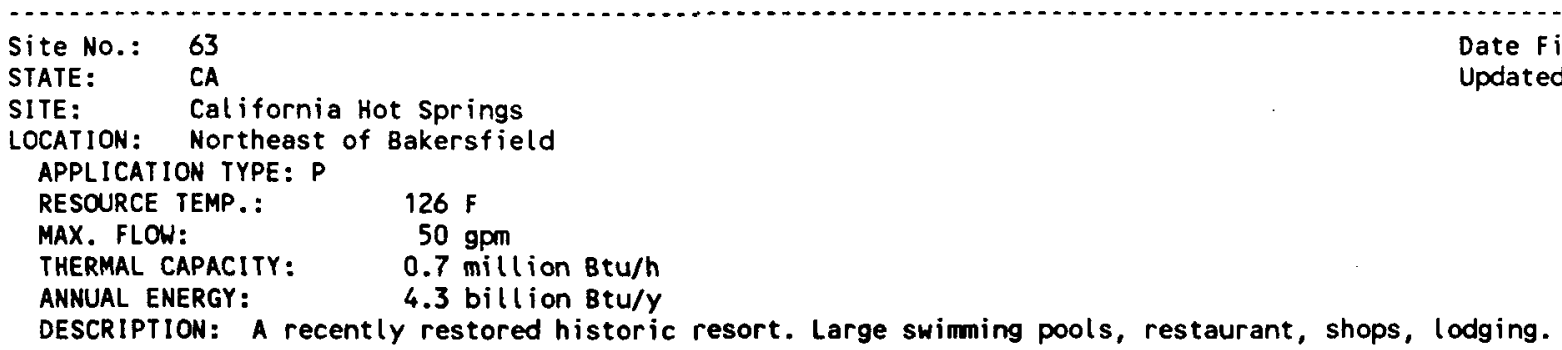

REFERENCE : Loam, 1985

Site No.: 64
STATE: $\quad$ CA
SITE: $\quad$ Calisoga High School
LOCATION: Calistoga
APPLICATION TYPE: S
RESOURCE TEMP.: $\quad 200 \mathrm{~F}$
MAX. FLOW:
THERMAL CAPACITY: $\quad 30 \mathrm{gpm}$
ANNUAL ENERGY: $\quad 3.0 \mathrm{mill}$ ion Btu/h
DESCRIPTION: The school has been using a down hole heat exchanger for space heating of one wing for the past 5 years.
Peak load of 150,000 Btu/hr. Construction of a new school which will be entirely geothermally heated,
including domestic hot water and showers, will start during late summer of 1988. The load of 3 million
Btu/hr is an estimate for the new school.

REFERENCE: Culver, 1988

Site NO.: 65

STATE: $\quad C A$

SITE: $\quad$ Keough Hot Springs

LOCATION: $10 \mathrm{mi} . \mathrm{S}$. Of Bishop, InYo Co.

APPLICATION TYPE: $P$

RESOURCE TEMP.: $\quad 128 \mathrm{~F}$

MAX. FLOW: $\quad 528 \mathrm{gpm}$

THERMAL CAPACITY: $\quad 0.5 \mathrm{mill}$ ion Btu/h

ANNUAL ENERGY: $\quad 3.9$ billion Btu/Y

DESCRIPTION: An older hot springs resort with swimming pool. The bathhouses (tubs) are closed. Food and RV hookups available.
Date Filed: $05 / 26 / 88$ Updated: 
APPLICATION TYPE: $P$

RESOURCE TEMP.:

MAX. FLOW:

THERMAL CAPACITY:

$106 \mathrm{~F}$

35 gpm

ANNUAL ENERGY:

$0.5 \mathrm{mill}$ ion Btu/h

DESCRIPTION: Originally the site of an indian village and still owned by the Aqua Caliente Tribe, as is all of the land in Palm Springs. Springs supply water for pools and spas in a large deluxe resort.

REFERENCE: CDMG, 1982; Loam, 1985

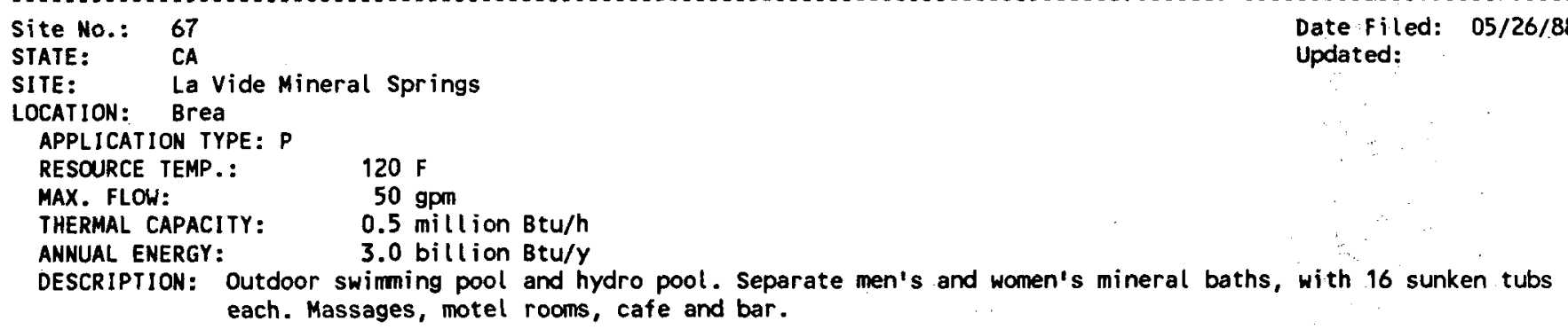

REFERENCE : LOAm, 1985

Site NO.: $\quad 68$
STATE: $\quad$ CA
SITE: $\quad$ Orr Hot Springs

REFERENCE: Loam, 1985; Culver, 1988

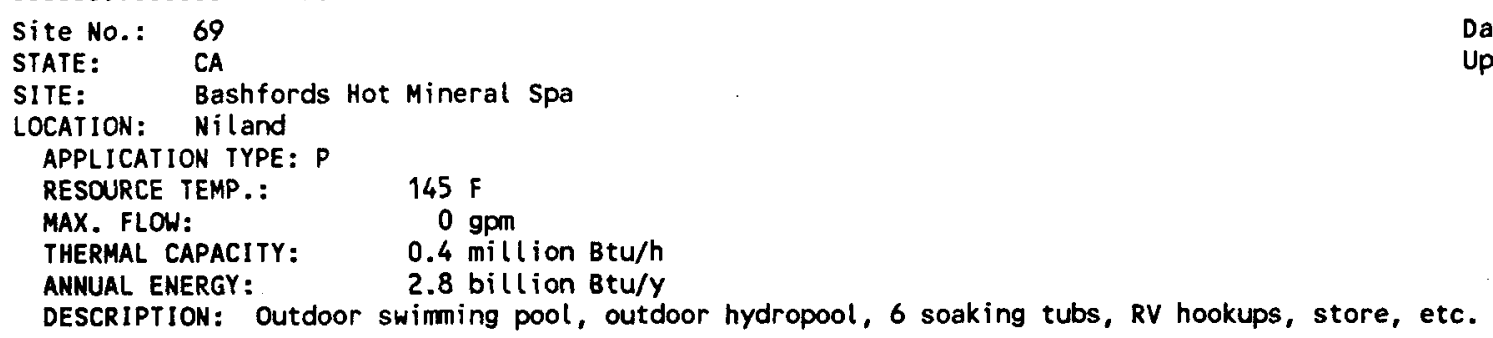

REFERENCE: LOam, 1985

Site NO.: 70
STATE: $\quad$ CA
SITE: $\quad$ White Suphur Springs




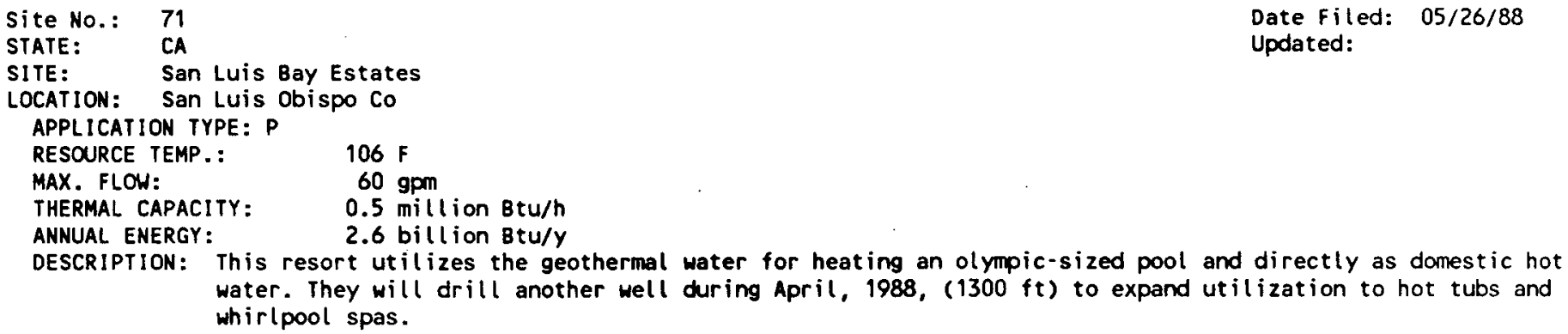

Site NO.: 72
STATE: $\quad$ CA
SITE: $\quad$ SUrPrise Valley Hospial
LOCATION: Cedarville
APPLICATION TYPE: P
RESOURCE TEMP.: $\quad 108 \mathrm{~F}$
MAX. FLOW:
THERMAL CAPACITY: $\quad 0.7 \mathrm{gPm}$
ANNUAL ENERGY: $\quad 2.2$ billion Btu/h
DESCRIPTION: Geothermal is cascaded from the elementary school and used for heating domestic hot water and make up
$\quad$ air pre heat which is a major load in a hospital.

REFERENCE: Culver, 1988; Chitwood, 1988

Site NO.: $\quad 73$
STATE: $\quad$ CA
SITE: $\quad$ LDS Church
LOCATION: SUSanville
APPLICATION TYPE: $\mathrm{S} \quad 128 \mathrm{~F}$
RESOURCE TEMP.: $\quad 90 \mathrm{gpm}$
MAX. FLOW:
THERMAL CAPACITY: $\quad 0.9 \mathrm{mill}$ ion Btu/h
ANNUAL ENERGY: $\quad 2.0 \mathrm{billion}$ Btu/y
DESCRIPTION: This 20,000 sq. ft. complex has been heated for a number of years. The well is $450 \mathrm{ft}$. deep. Discharge
is to surface disposal, but there is some consideraton of not permitting the discharge in the future.
The church is investigating the options of injection or hookup to the city system.

REFERENCE: Culver, 1988

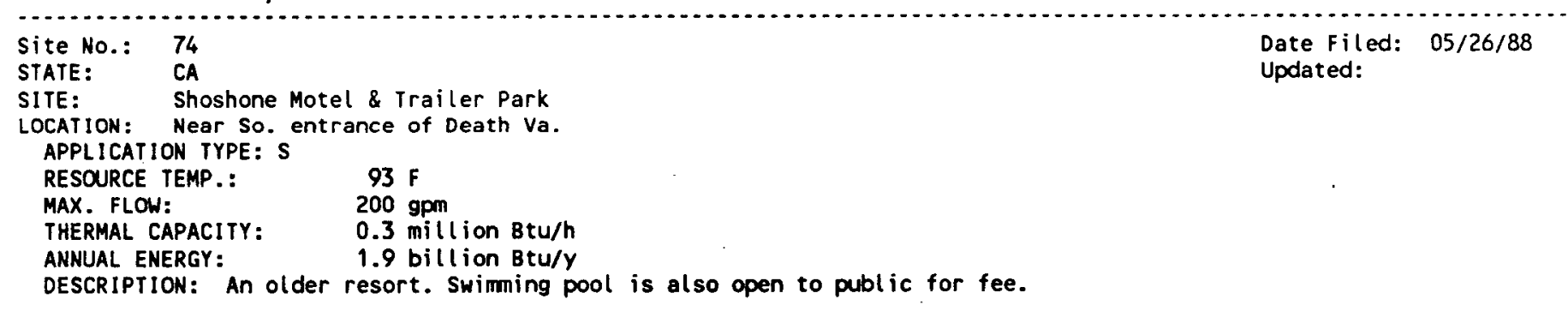

REFERENCE: LOam, 1985

Site NO.: 75
STATE:
SITE: CA


DESCRIPTION: Spring. water at about $180 \mathrm{~F}$ is mixed with cool spring water to supply a pool $(30 \times 60)$. The use has cont inued since the late 1800 's. Since the formation of the park in the 1950's the facility has been operated under lease from the park service. No other facilities at the guest ranch are heated.

REFERENCE: Vance, 1985

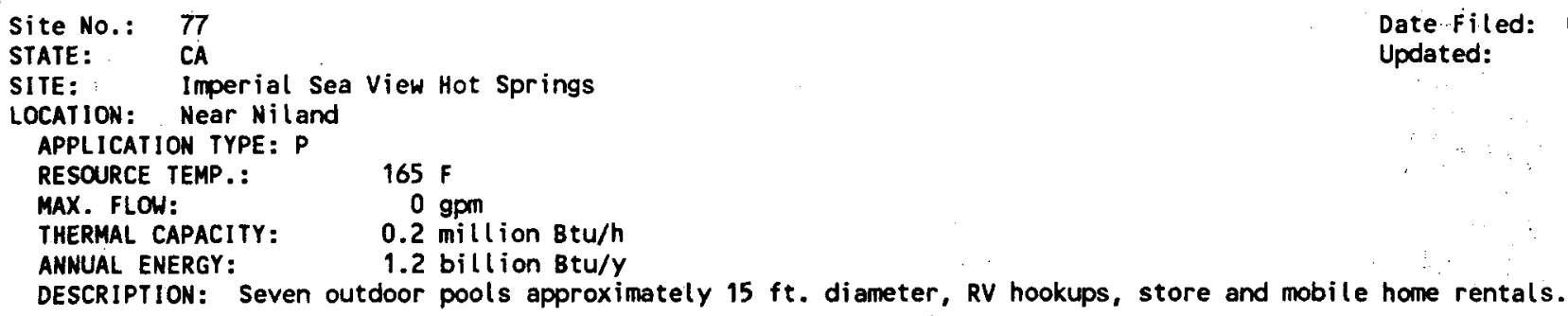

REFERENCE : Loam, 1985

Site No.: $\quad 78$
STATE: $\quad$ CA
SITE: MONO Hot Springs
LOCATION: $80 \mathrm{mi}$ NE of Fresno
APPLICATION TYPE: P $\quad 107 \mathrm{~F}$
RESOURCE TEMP.: $\quad 53 \mathrm{gpm}$
MAX. FLOW:
THERMAL CAPACITY: $\quad 0.2 \mathrm{mill}$ ion Btu/h
ANMUAL ENERGY: $\quad 1.0$ billion Btu/Y
DESCRIPIION: A commercial bath house with indoor (pvt.) and outdoor tubs.

REFERENCE : Loam, 1985

Site NO.: $\quad 79$
STATE: $\quad$ CA
SITE: $\quad$ Indian Valley Hospital
LOCATION: Greenville
APPLICATION TYPE: S
RESOURCE TEMP.:
MAX. FLOW: $\quad 117 \mathrm{~F}$
THERMAL CAPACITY: $\quad 0.5 \mathrm{gpm}$
ANNUAL ENERGY: $\quad 0.5 \mathrm{mill}$ ion Btu/h
DESCRIPTION: This small hospital complex was heated by electricity. A heat pump system was designed and installed,
but failed to meet public heal th dept. standards. A re-design eleminating the heat pumps and using heat
exchangers is under construction.

REFERENCE: Rafferty, 1988

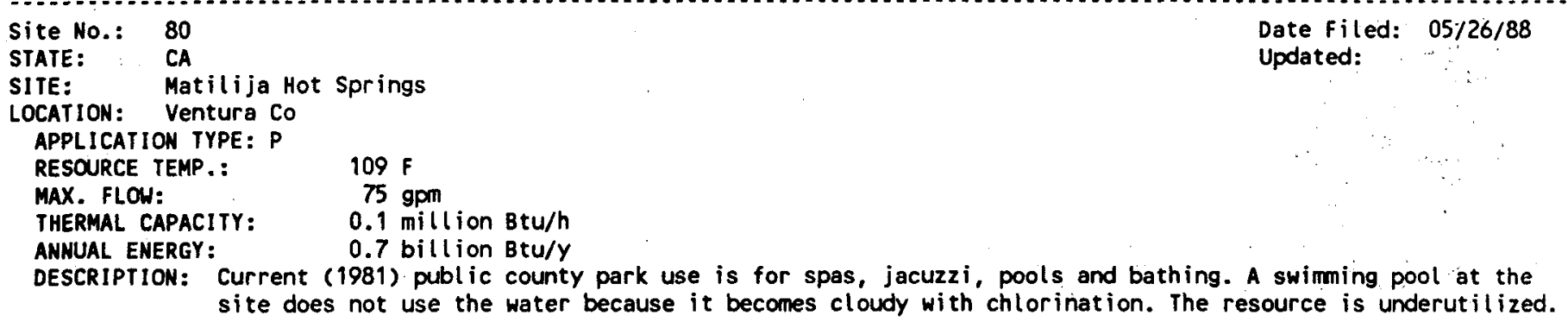


DESCRIPIION: Once the site of a hotel having accommodations for 100 people. Now a private recreation association resort with a few cabins. Geothermal water is used in the pool.

REFERENCE: CDMG, 1982

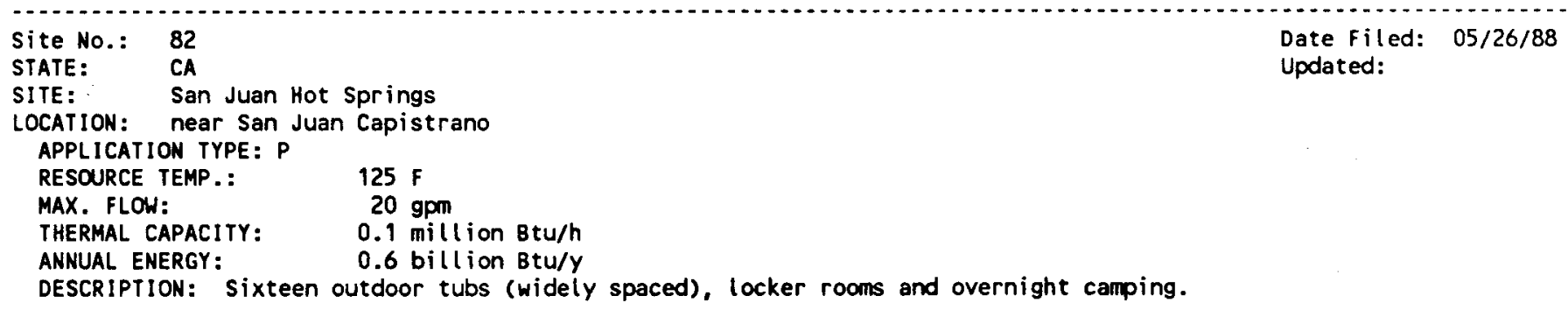

REFERENCE: Loam, 1985

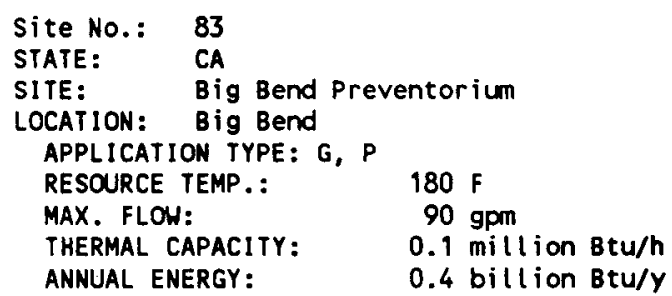

DESCRIPTION: An old historical resort now rehabilitated and operated by the co-op Essene Cormunity. Natural springs supply mineral tubs and pools. Cabins, massages, RV park, seminars. Greenhouse for vegetables.

REFERENCE : Loam, 1985

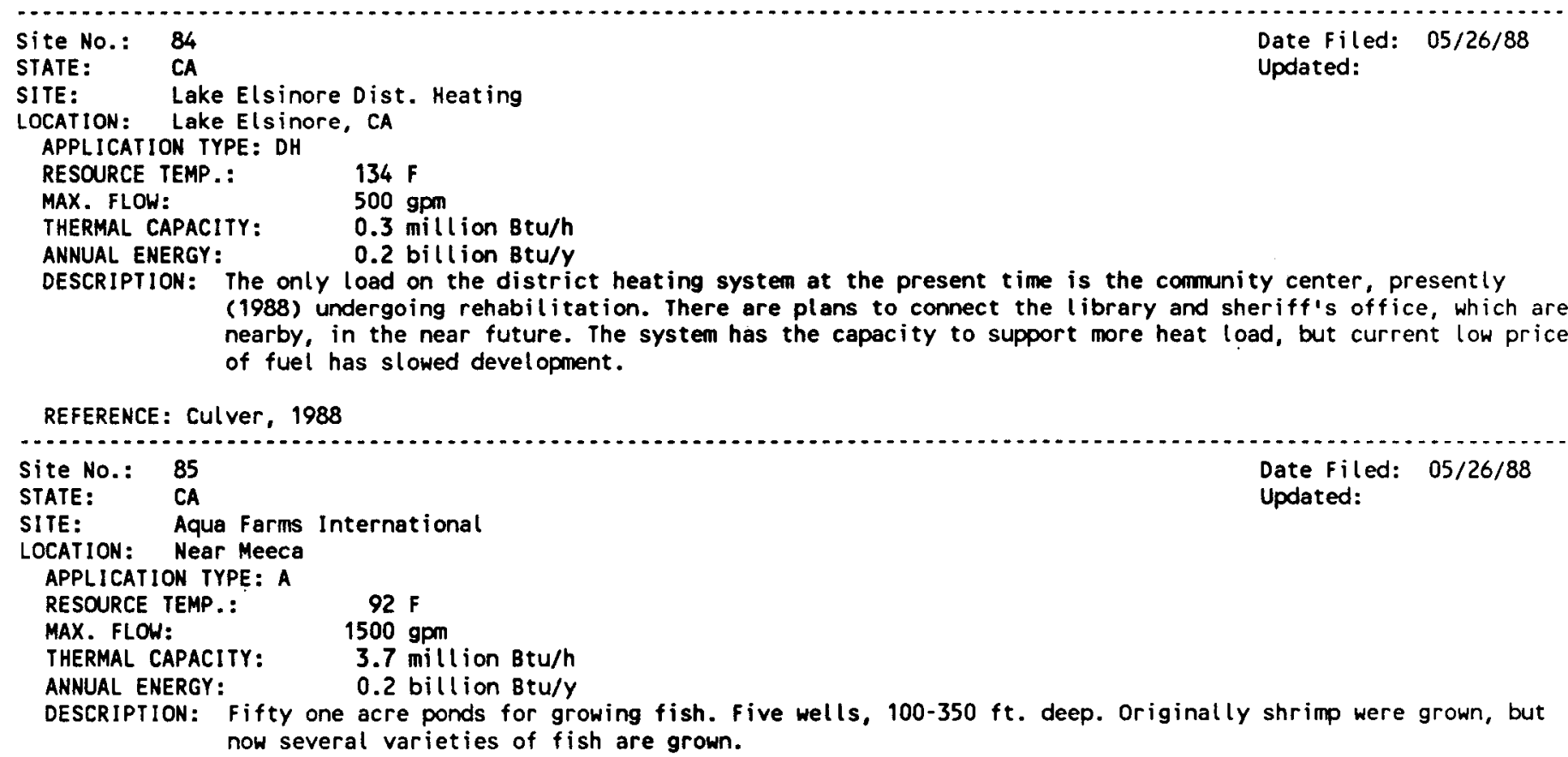


LOCATION: $80 \mathrm{mi}$ E. of San Diego

APPLICATION TYPE: P

RESOURCE TEMP.: $97 \mathrm{~F}$

MAX. FLOW:

THERMAL CAPACITY: $\quad 0.0 \mathrm{mill}$ ion Btu/h

ANNUAL ENERGY: $0.1 \mathrm{bill}$ ion Btu/y

OESCRIPTION: An older motel, spa, rooms, restaurant, bar and sauna.

REFERENCE : Loam, 1985

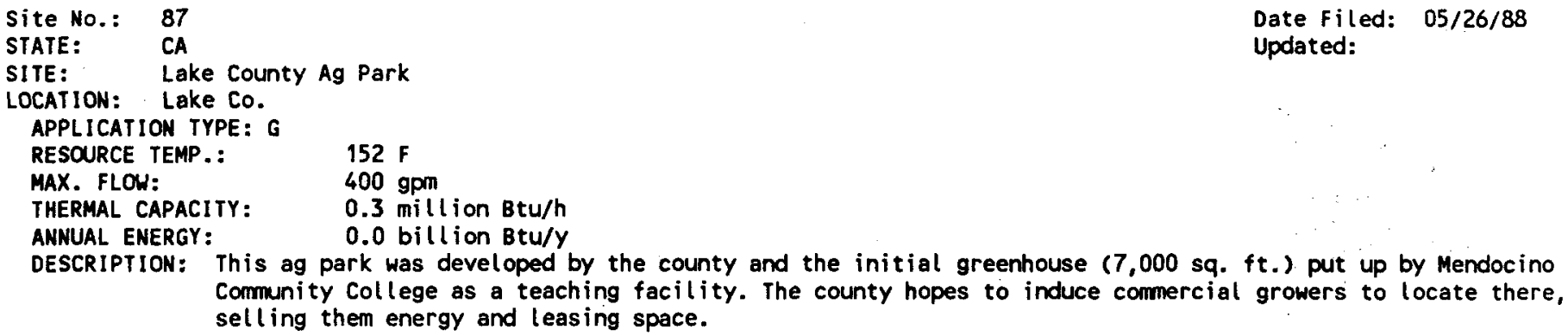

DESCRIPTION: This ag park was developed by the county and the initial greenhouse (7,000 sq. $f t$. ) put up by Mendocino community college as a teaching facility. The county hopes to induce commercial growers to locate there, selling them energy and leasing space.

REFERENCE : Dillinger, 1988

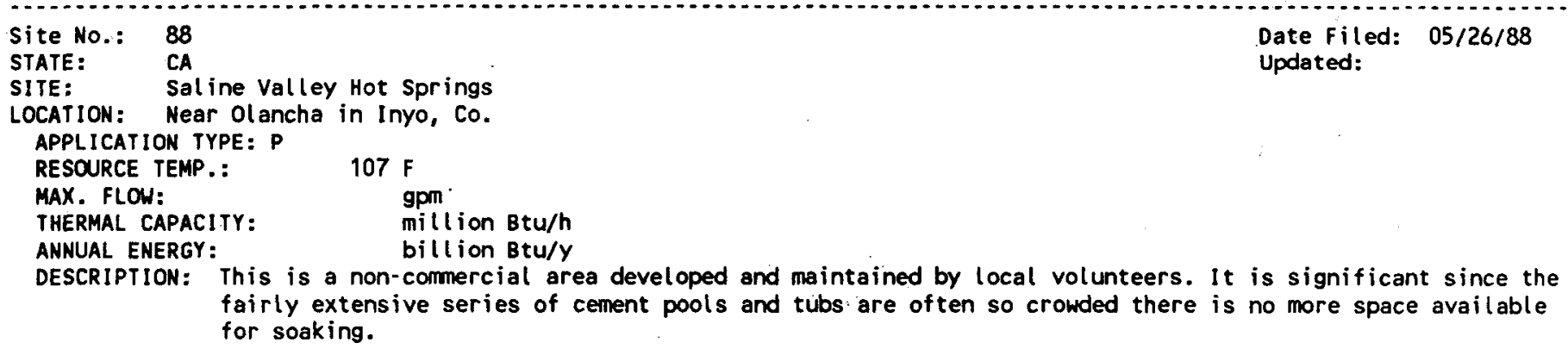

REFERENCE : Loam, 1985

Site No.: 89

STATE: CA

SITE: $\quad$ Reds Meadow Hot Springs

Date Filed: $05 / 26 / 88$

LOCATION: Devils Postpile Natl Monument

APPLICATION TYPE: P

RESOURCE TEMP.: $115 \mathrm{~F}$

MAX. FLOH: 15 gPm

THERMAL CAPACITY: million Btu/h

ANNUAL ENERGY: billion Btu/y

DESCRIPTION: Tubs and showers at a U.S. Forest Service Campground

REFERENCE : Loam, 1985

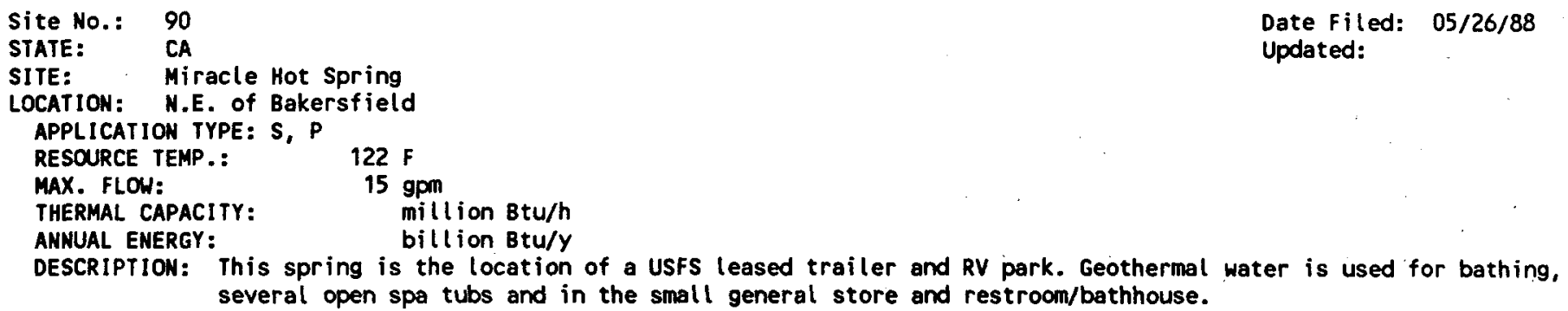


DESCRIPTION: This old resort hotel is currently being retrofit to use geothermal from an artesian well. The pool retrofit has been completed and work is underway on the hotel. Heat loads can be determined when work is further along.

REFERENCE: Fisher, 1988; Cutbertson, 1988

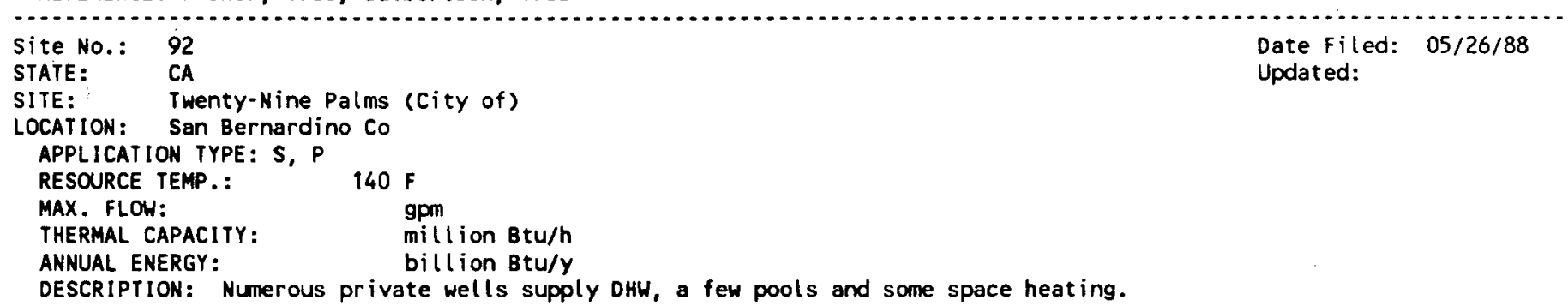

REFERENCE: COMG, 1982

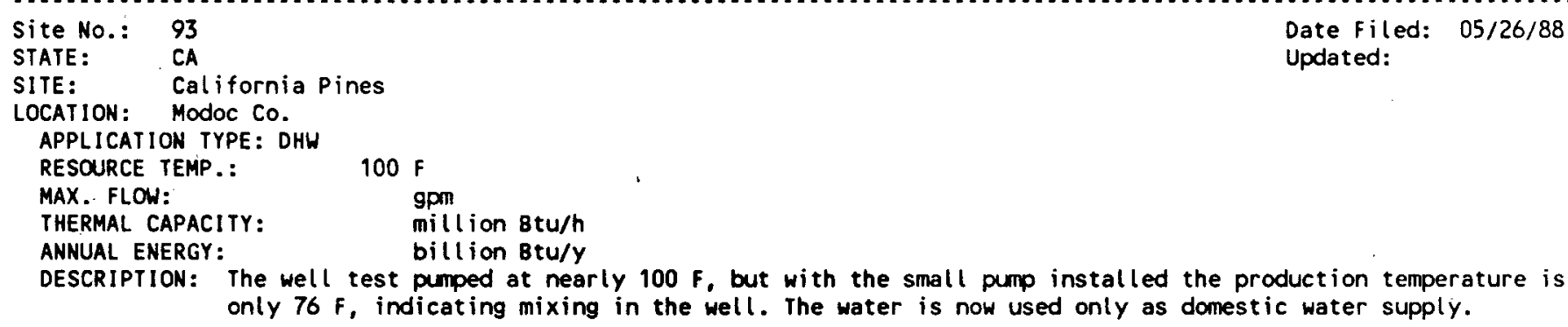

REFERENCE : Davenport, 1988

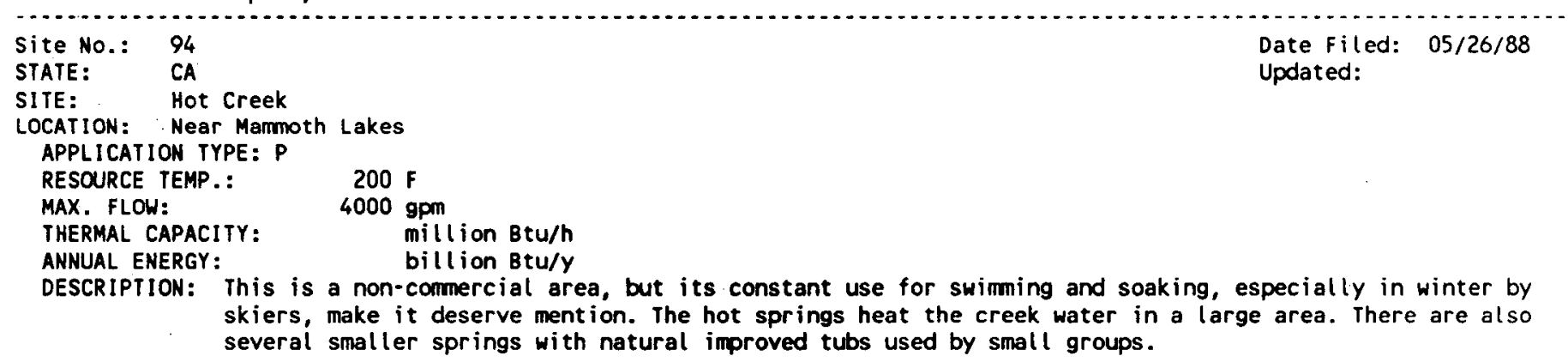

REFERENCE: Loam, 1985; Culver, 1988

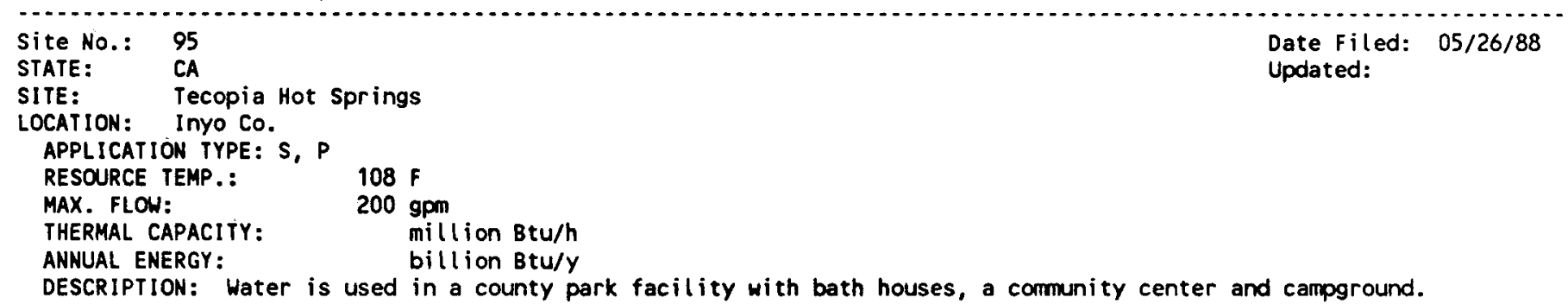


LOCATION:

APPLICATION TYPE: S, P

RESOURCE TEMP.: 142

MAX. FLOW: $\quad 300 \mathrm{gpm}$

THERMAL CAPACITY: million Btu/h

ANNUAL ENERGY: billion Btu/Y

DESCRIPTION: As of 1981, a ski resort was being developed. Geothermal is to be used for a pool, spas and perhaps space and DHW heating.

REFERENCE : COMG, 1982

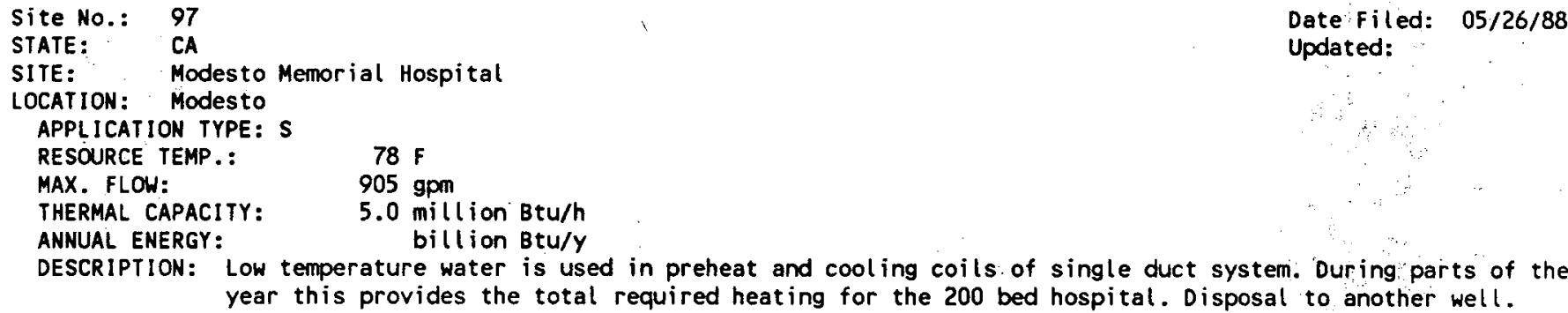

Site No.: 98
STATE: $\quad$ CA
SITE: $\quad$ Nevares Springs
LOCATION: InYO CO. at Death Valley (VC)
APPLICATION TYPE: DHW
RESOURCE TEMP.:
MAX. FLOW:
THERMAL CAPACITY: $\quad 104 \mathrm{~F}$
ANNUAL ENERGY:
DESCRIPTION: Water is used as domestic hot water in National Park Service buildings, employee residences, and at the
visitors center.

REFERENCE : Loam, 1985

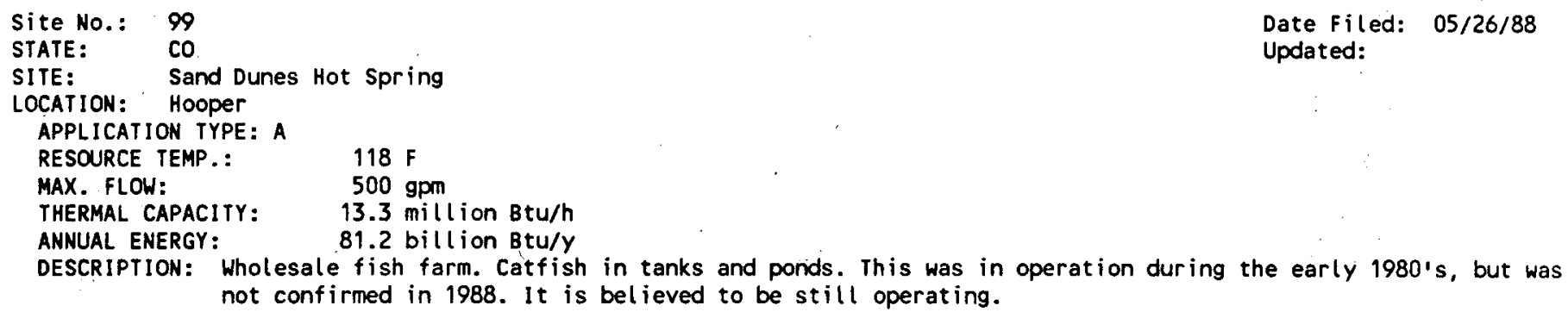




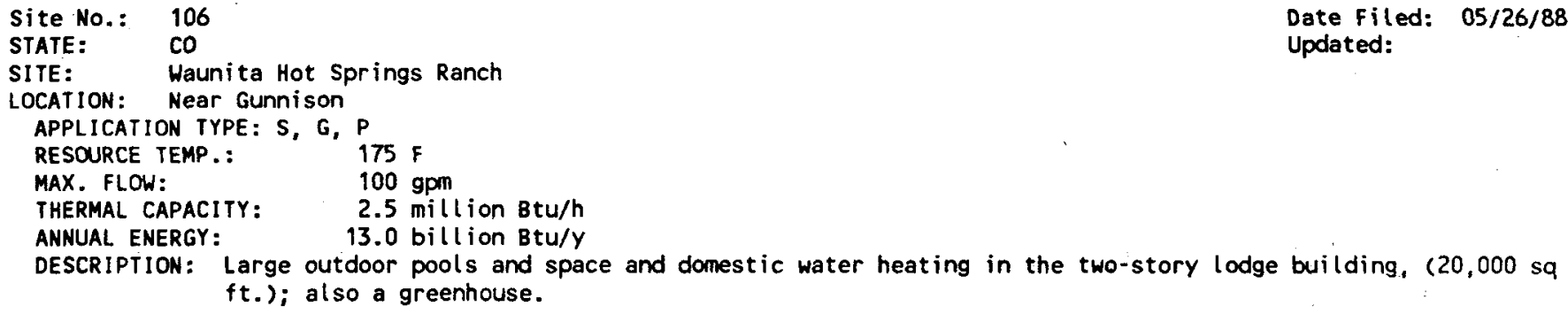

Site NO.: 107
STATE: $\quad$ CO
SITE: $\quad$ All of State
LOCATION:
APPLICATION TYPE: HP
RESOURCE TEMP.: $\quad 52 \mathrm{~F}$
MAX. FLOW:
THERMAL CAPACITY: $\quad 7.2 \mathrm{gpm}$
ANNUAL ENERGY:
DESCRIPTION: An estimated 200 vertical closed loop earth coupled heat pump wells are used for space heating and
Cooling.

REFERENCE: Ellis, 1988

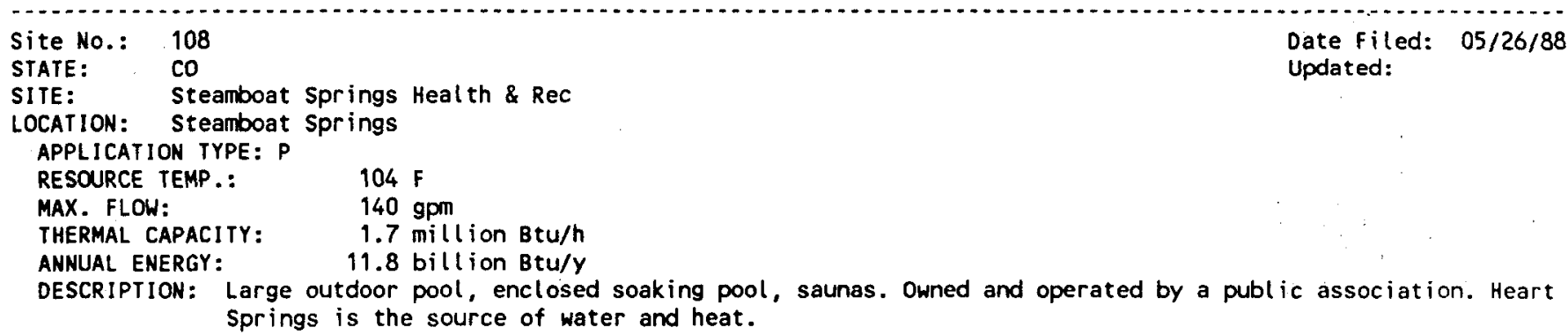

REFERENCE: Loam, 1980; Rold, 1988

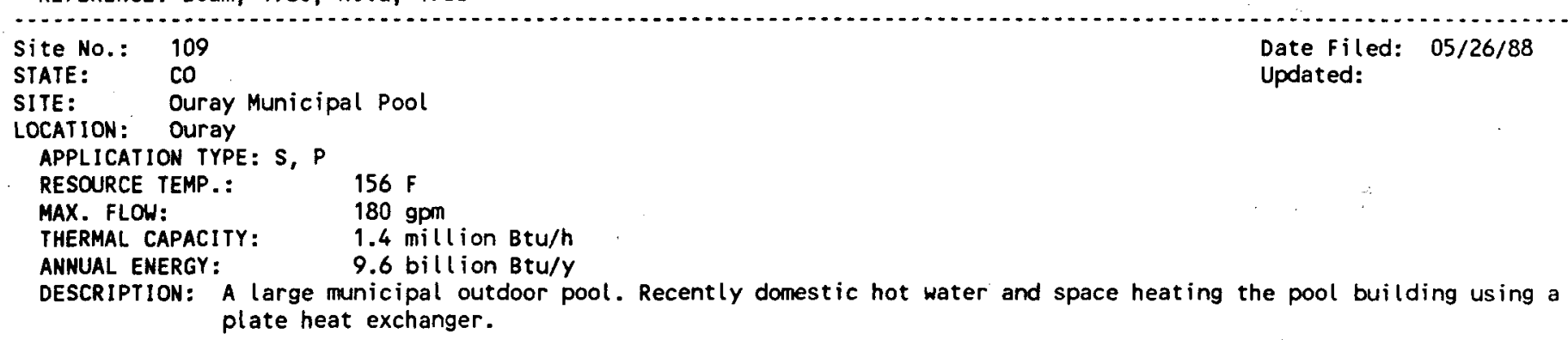

REFERENCE : Culver, 1988

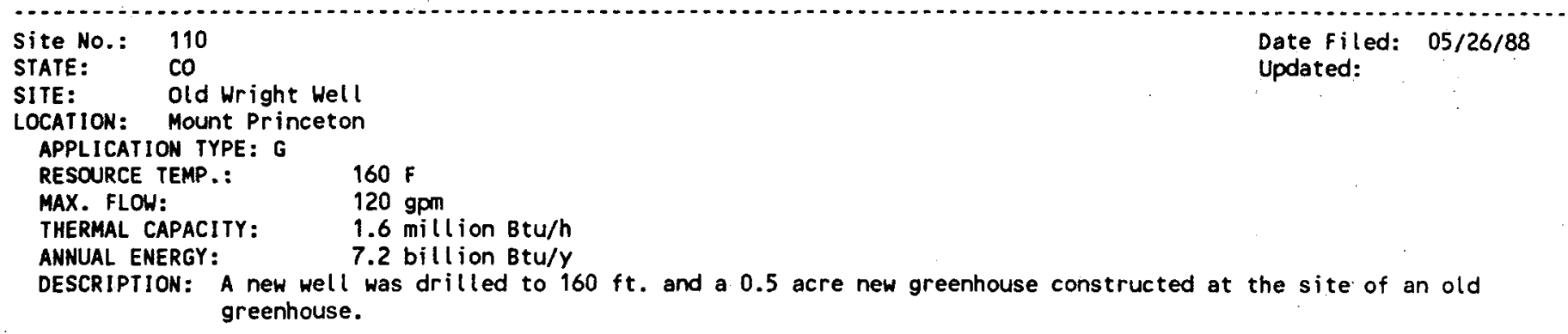


SITE: $\quad$ Jump steady Resort

LOCATION: Buena Vista

APPLICATION TYPE: S, P

RESOURCE TEMP.: $119 \mathrm{~F}$

MAX. FLOW: $90 \mathrm{gPm}$

THERMAL CAPACITY: $\quad 1.0 \mathrm{mill}$ ion Btu/h

ANNUAL ENERGY: $\quad 7.0$ billion Btu/Y

DESCRIPTION: A smaller resort, with outdoor swimming pool, soaking pools and tubs. Water piped to all rooms for showers and private tubs. Also, at least two nearby homes are heated using water from the springs.

REFERENCE : Loam, 1980; Rold, 1988

Site No.: 112
STATE: $\quad$ CO
SITE: $\quad$ Cottonwood Hot Springs
LOCATION: Hest of Buena Vista
APPLICATION TYPE: S $\quad 119 \mathrm{~F}$
RESOURCE TEMP.: $\quad 10 \mathrm{gpm}$
MAX. FLOW:
THERMAL CAPACITY: $\quad 0.2 \mathrm{mill}$ ion Btu/h
ANNUAL ENERGY: $\quad 6.3$ billion Btu/y
DESCRIPTION: Four homes are heated utilizing the springs.

REFERENCE: Rold, 1988

$\begin{array}{ll}\text { Site No.: } & 113 \\ \text { STATE: } & \text { CO } \\ \text { SITE: } & \text { Valley View Hot Springs } \\ \text { LOCATION: Near Villa Grove } \\ \text { APPLICATION TYPE: P } \\ \text { RESOURCE TEMP.: } & 99 \mathrm{~F} \\ \text { MAX. FLOW: } & 180 \mathrm{gpm} \\ \text { THERMAL CAPACITY: } & 0.8 \mathrm{mill} \text { ion Btu/h } \\ \text { ANNUAL ENERGY: } & 5.7 \mathrm{bill} \text { ion } \mathrm{Btu} / \mathrm{y}\end{array}$

DESCRIPTION: Two large outdoor pools, seven soaking pools, sauna. A membership resort.

REFERENCE: Loam, 1980; Rold, 1988

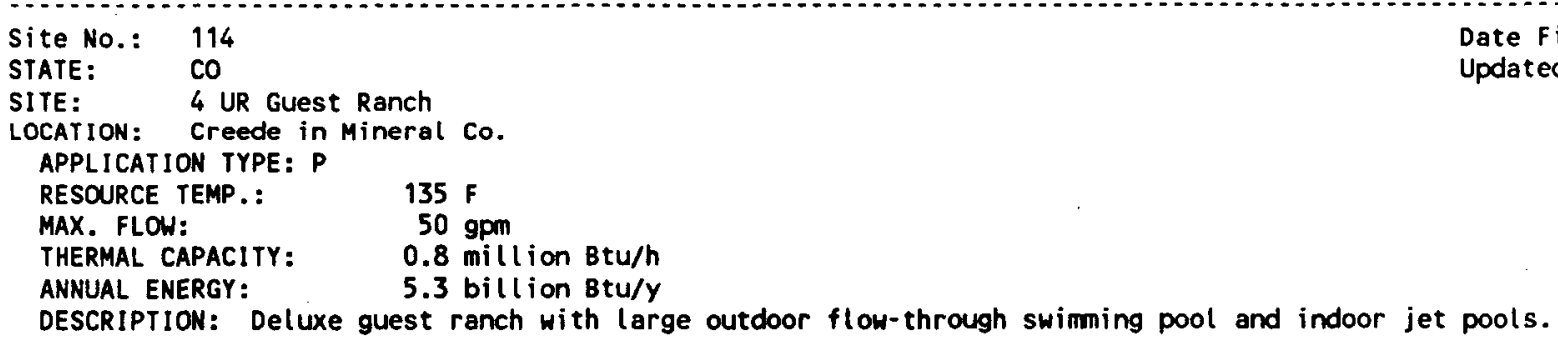

REFERENCE: Loam, 1980; Rold, 1988

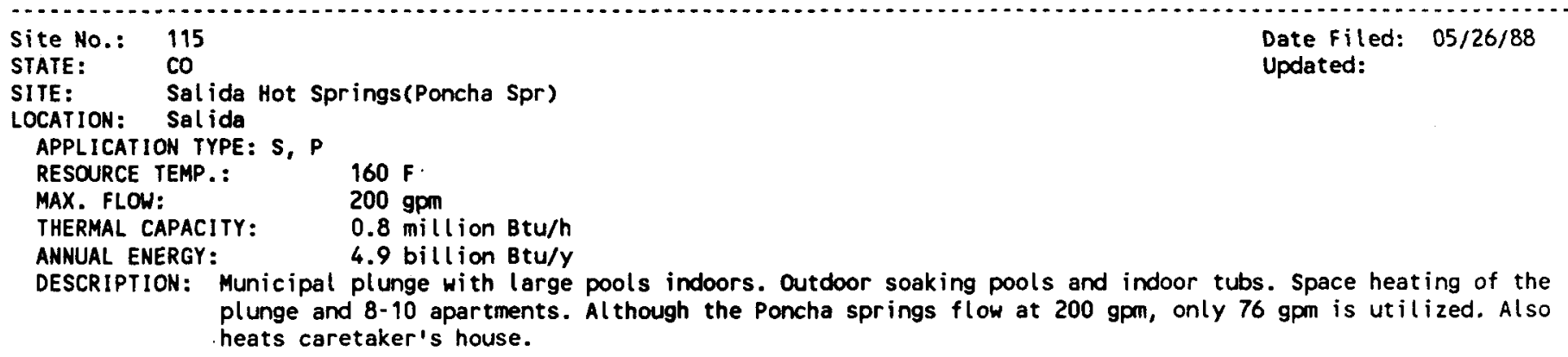


SITE: $\quad$ Hot Sulphur Springs

LOCATION: Hot Sulphur Springs

APPLICATION TYPE: S, P

RESOURCE TEMP.:

MAX. FLOW:

$111 \mathrm{~F}$

$0.7 \mathrm{mill}$ ion Btu/h

DESCRIPTION: Large outdoor swimming pool, indoor soaking pools, space heating and laundry. Water from 5-10 springs is collected and piped to the facility.

REFERENCE: Loam, 1980; Rold, 1988

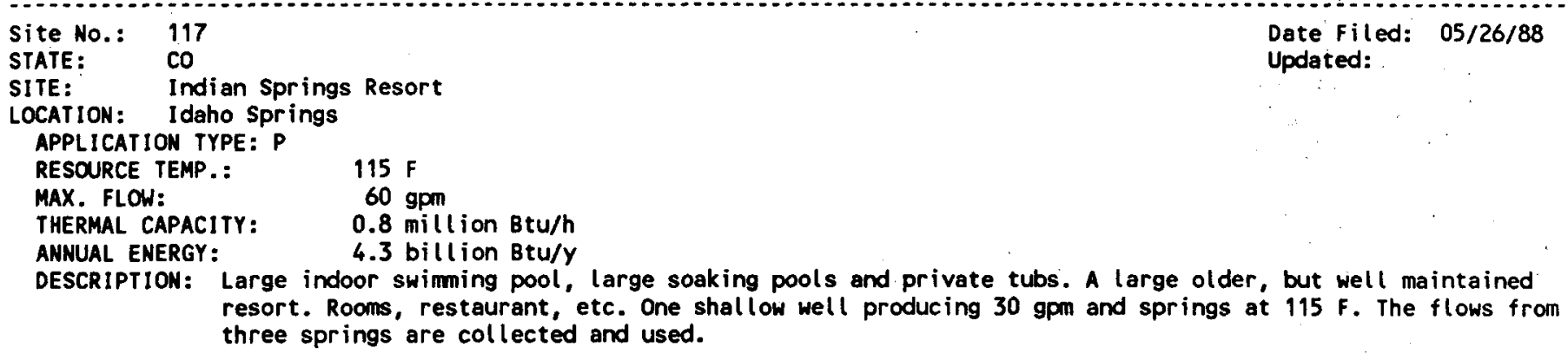

REFERENCE: Loam, 1980; Rold, 1988

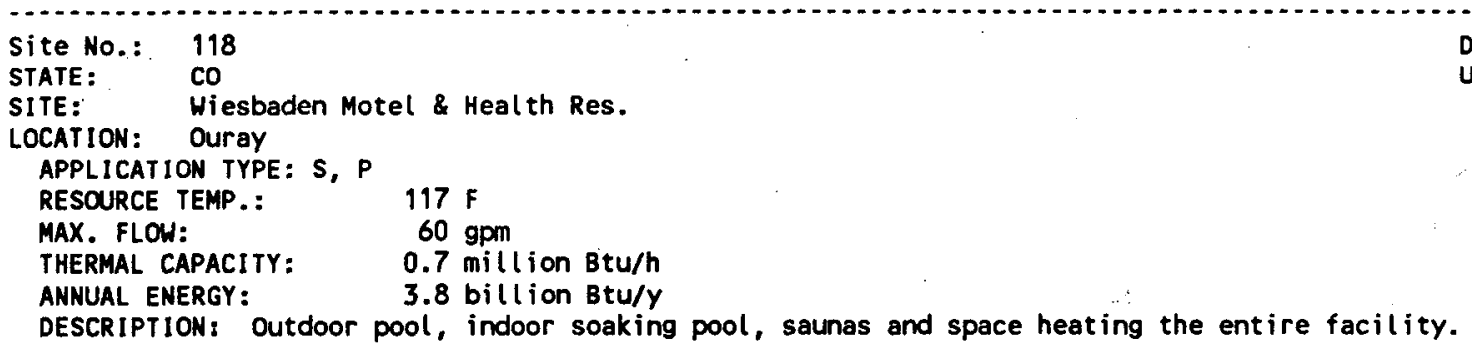

REFERENCE: Hut Trer, 1988

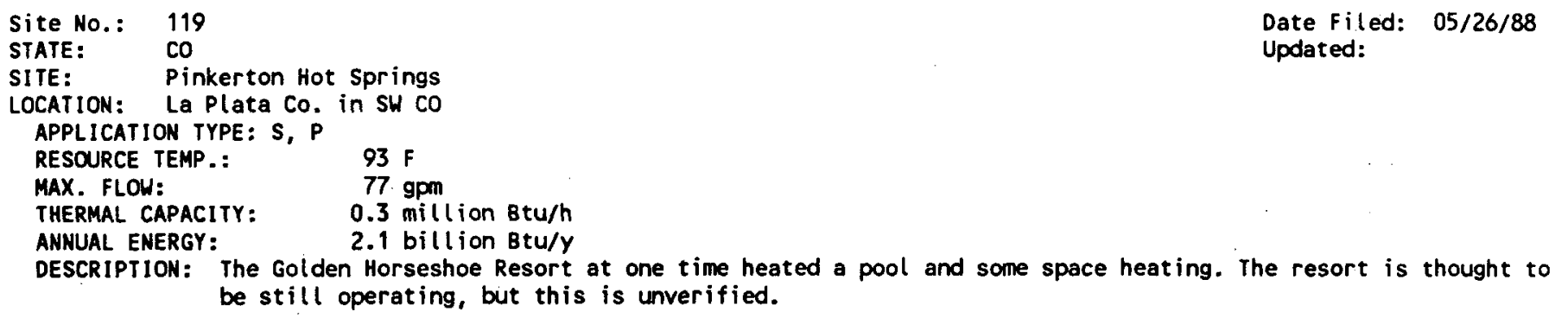

REFERENCE: Rold, 1988

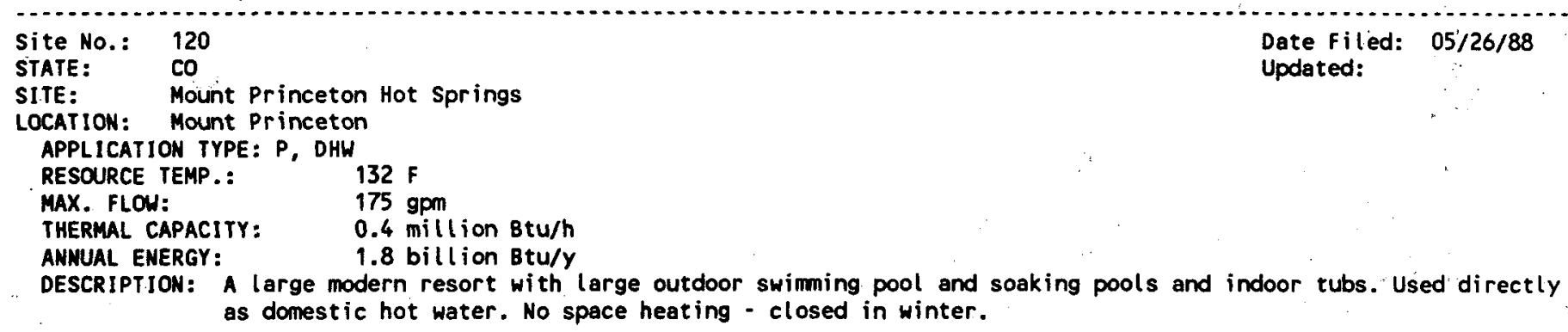


SITE: $\quad$ Twin Peaks Motel

LOCATION: OURAY

APPLICATION TYPE: S, P

RESOURCE TEMP.: $\quad 110 \mathrm{~F}$

MAX. FLOW: $\quad 15 \mathrm{gpm}$

THERMAL CAPACITY: $\quad 0.2 \mathrm{mill}$ ion Btu/h

ANNUAL ENERGY: $\quad 1.1 \mathrm{bill}$ ion $B t U / Y$

DESCRIPTION: Used directly for space heating and heating a small swiming pool

REFERENCE: Huttrer, 1988

$\begin{array}{ll}\text { Site No.: } & 122 \\ \text { STATE: } & \text { CO } \\ \text { SITE: } & \text { Box Canyon Motel } \\ \text { LOCATION: Ouray } & \\ \text { APPLICATION TYPE: S, P } & \\ \text { RESOURCE TEMP.: } & 110 \mathrm{~F} \\ \text { MAX. FLOW: } & 15 \mathrm{gpm} \\ \text { THERMAL CAPACITY: } & 0.2 \mathrm{mill} \text { ion Btu/h } \\ \text { ANNUAL ENERGY: } & 1.1 \mathrm{bill} \text { ion Btu/y }\end{array}$

OESCRIPIION: Used directly for space heating of a portion of the motel and for soaking tubs.

Date Filed: $05 / 26 / 88$ Updated:

REFERENCE: Huttrer, 1988

Site NO.: 123
STATE: $\quad$ CO
SITE:
LOCATION: Mount Princeton Area
APPLICATION TYPE: $\mathrm{S}, \mathrm{P}$
RESOURCE TEMP.:
MAX. FLOW:
THERMAL CAPACITY: $\quad 0.3 \mathrm{gpm}$
ANNUAL ENERGY: $\quad 0.6$ billion Btu/h
DESCRIPTION: In the Mount Princeton area there are several applications in additon to the Mount Princeton Hot Springs
Resort. A church group operates Young Life Camp, Woolmington Resort heats at least a pool, using a well
and there are at least 2 homes and 2 motel pools heated. At the present, time little is known except
they do exist.

REFERENCE : Rold, 1988

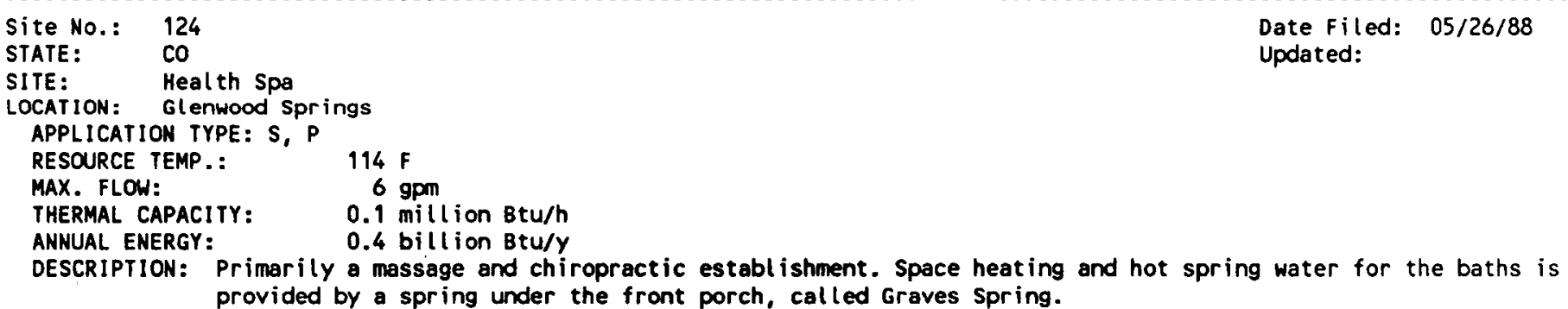

REFERENCE : Rold, 1988

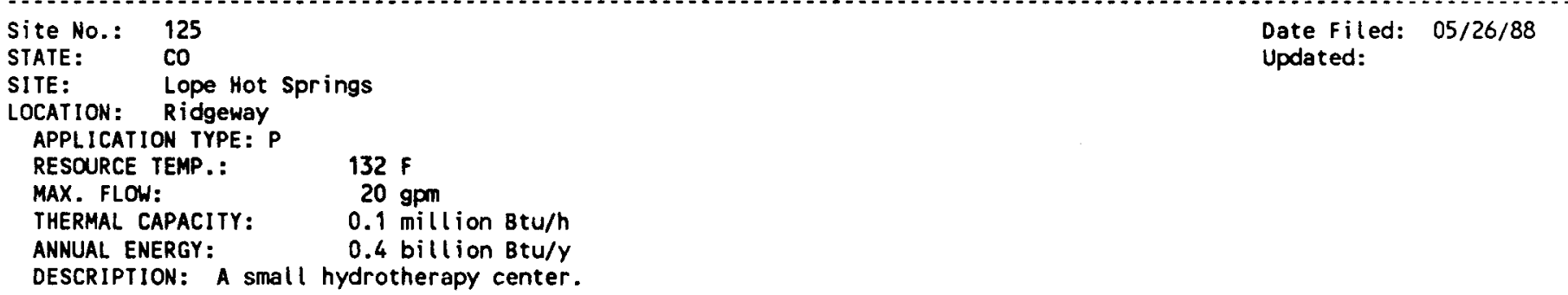


SITE: Cement Creek Ranch

LOCATION: Crested Butte N. of Gunnison

APPLICATION TYPE: P

RESOURCE TEMP.:

MAX. FLOW:

THERMAL CAPACITY:

AHNUAL ENERGY:

$78 \mathrm{~F}$

$70 \mathrm{gpm}$

$0.1 \mathrm{mill}$ ion Btu/h

0.4 billion Btu/y

DESCRIPTION: Flow through pool at guest ranch.

REFERENCE: Loam, 1980; Rold, 1988

Site NO.: $\quad 127$
STATE: $\quad$ CO
SITE: $\quad$ Dunton Hot Springs
LOCATION: Near Dolores
APPLICATION IYPE: P
RESOURCE TEMP.: $\quad 107 \mathrm{~F}$
MAX. FLOW:
THERMAL CAPACITY: $\quad 25 \mathrm{gpm}$
ANNUAL ENERGY: $\quad 0.1 \mathrm{million}$ Btu/h
DESCRIPTION: Smaller old resort. Indoor soaking tubs.

REFERENCE: LOan, 1980; Rold, 1988

Site NO.: 128
STATE: $\quad$ CO
SITE: $\quad$ Trip Hot Springs
LOCATION: LaPLata in SW Colorado
APPLICATION TYPE: G, P $111 \mathrm{~F}$
RESOURCE TEMP.: $\quad$ gpm
MAX. FLOW:
THERMAL CAPACITY: $\quad$ million Btu/h
ANNUAL ENERGY:
DESCRIPTION: There may be a small greenhouse, some buildings and a pool heated using spring water. These may actually
be at Pinkerton Hot Springs.

REFERENCE: Rold,

Site No.: 129
STATE: $\quad$ CO
SITE:
LOCATION: Glenwood Springs Vapor Caves
APPLICATION TYPE: P
RESOURCE TEMP.:
MAX. FLOW:
THERMAL CAPACITY:
ANNUAL ENERGY:
DESCRIPTION: Cares with water and steam used as steam baths (separate mens and womens). No estimate of the beneficial
heat provided was made.

REFERENCE: Loam, 1980; Rold, 1988

Site NO.: 130
STATE: $\quad$ CO
SITE: $\quad \begin{aligned} & \text { Ouray District Heating } \\ & \text { LOCATION: OURay }\end{aligned}$
APPLICATION TYPE: DH
RESOURCE TEMP.: $\quad 156 \mathrm{~F}$
MAX. FLOW: $\quad 780 \mathrm{gpm}$
THERMAL CAPACITY: $\quad$ million Btu/h
ANMUAL ENERGY:
DESCRIPTION: A district heating system is being developed. To date 6 wells have been drilled, four with useable
$\quad$ temperatures and flows, and the $156 \mathrm{~F}$ spring will be utilized.


LOCATION: CanON City

APPLICATION TYPE: S

RESOURCE TEMP.:

MAX. FLOW: gpm

THERMAL CAPACITY: $\quad$ million $8 \mathrm{tu} / \mathrm{h}$

ANNUAL ENERGY: billion $B t u / y$

DESCRIPTION: Heating of a new prison was proposed and the prison was built to permit easy retrofit. To date, the resource has not been confirmed at the prision and wells have not been drilled. The heat ing of homes and pools in the area has been rumored, but not been confirmed - nor is it definately known there are not.

REFERENCE : Rold, 1988

Site NO.: 132
STATE: $\quad$ DE
SITE: : All of State
LOCATION:
APPLICATION TYPE: HP
RESOURCE TEMP.:
MAX. FLOW:
THERMAL CAPACITY: $\quad 47 \mathrm{~F}$
ANNUAL ENERGY: $\quad 20.5 \mathrm{gPm}$
DESCRIPTION: An estillion Btu/h
$\quad 35.9$ billion Btu/Y
space heating.

REFERENCE: Ellis, 1988

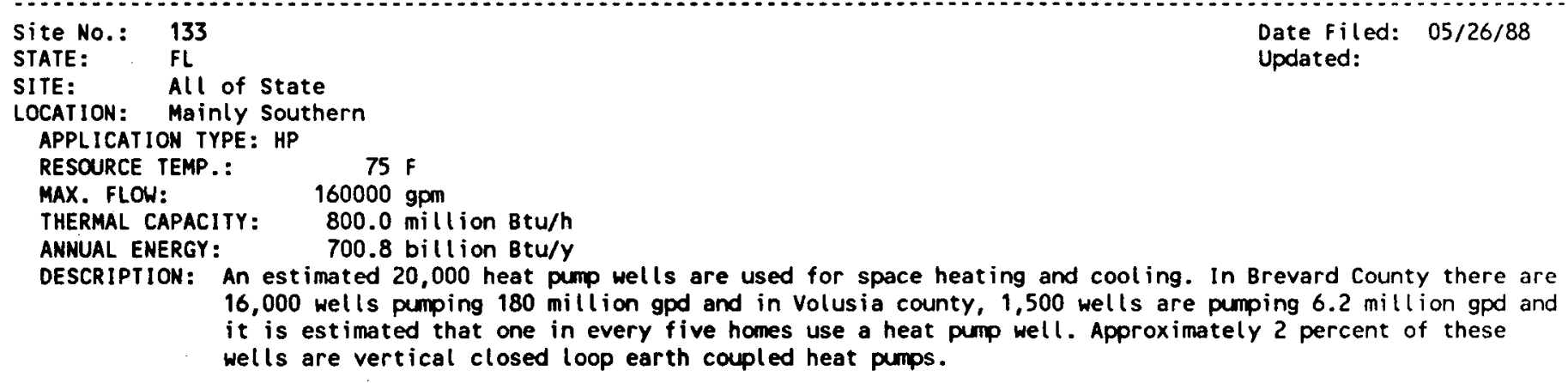

REFERENCE : MCCray, 1988

Site NO.: 134
STATE: $\quad$ FL
SITE: $\quad$ Patrick Air Force Base
LOCATION: Melbourne
APPLICATION TYPE: HP
RESOURCE TEMP.:
MAX. FLOW: $\quad 72 \mathrm{~F}$
THERMAL CAPACITY: $\quad 3000 \mathrm{gmm}$
ANNUAL ENERGY: $\quad 39.6 \mathrm{million} \mathrm{Btu/h}$
DESCRIPTION: A district heating and cooling system provided by groundwater-source heat pumps using 38 production and
injection wells in a loop. This system realized a large savings after converting from electric cool ing.

REFERENCE: Frazee, 1988

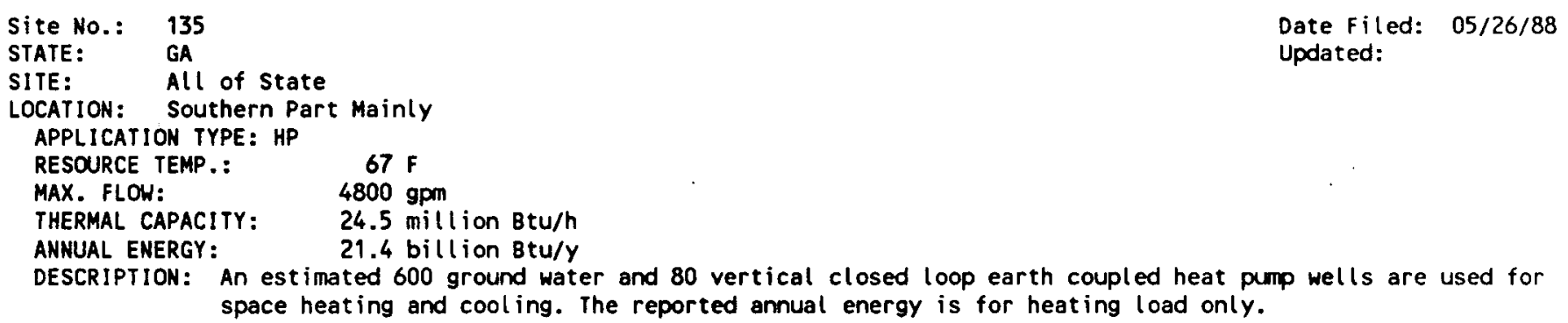




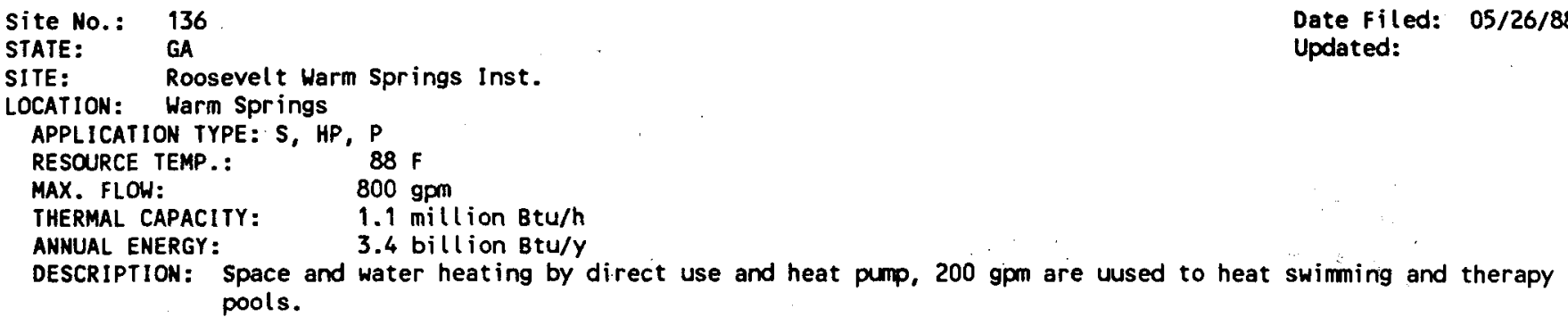

REFERENCE: Aiken, 1988

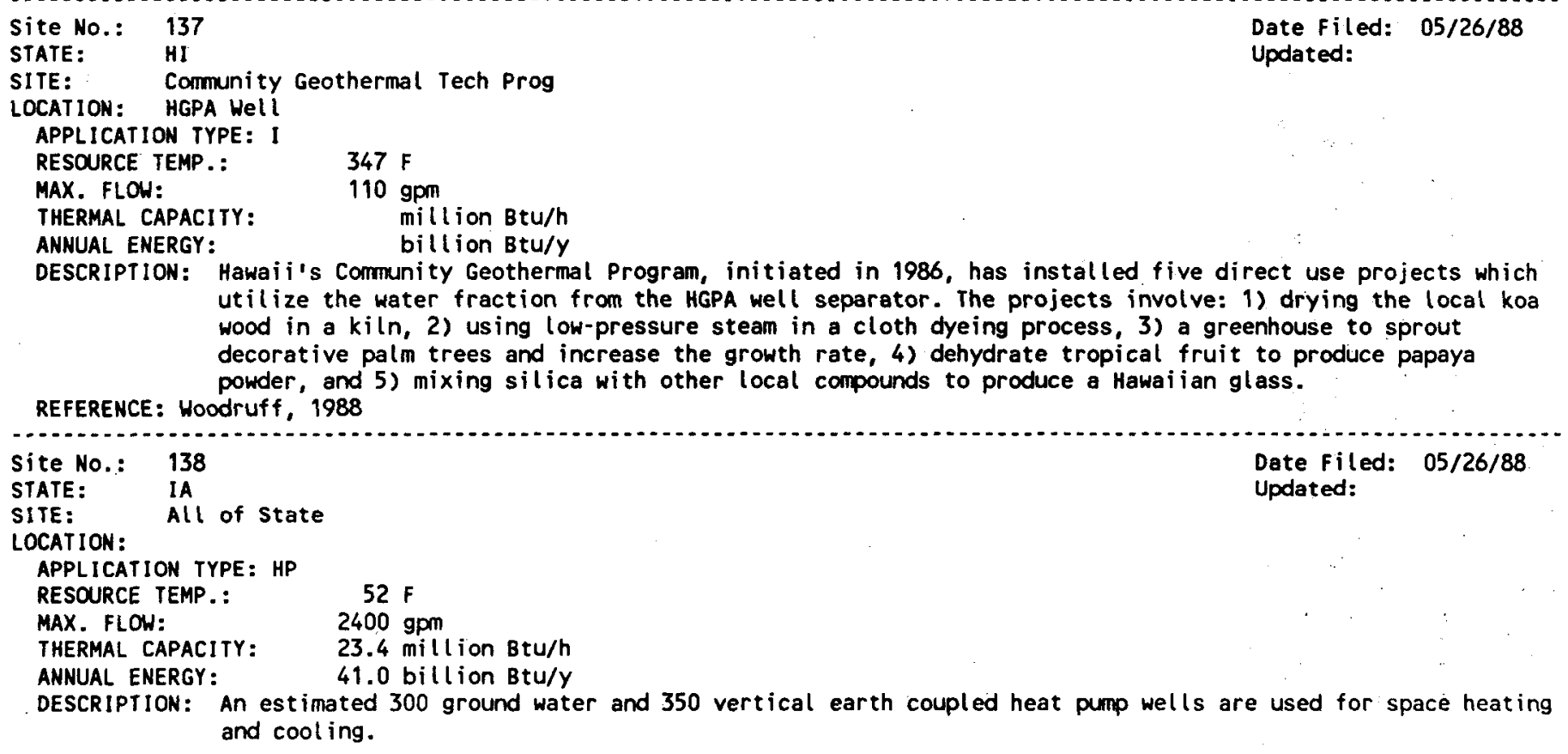

REFERENCE: Ellis, 1988

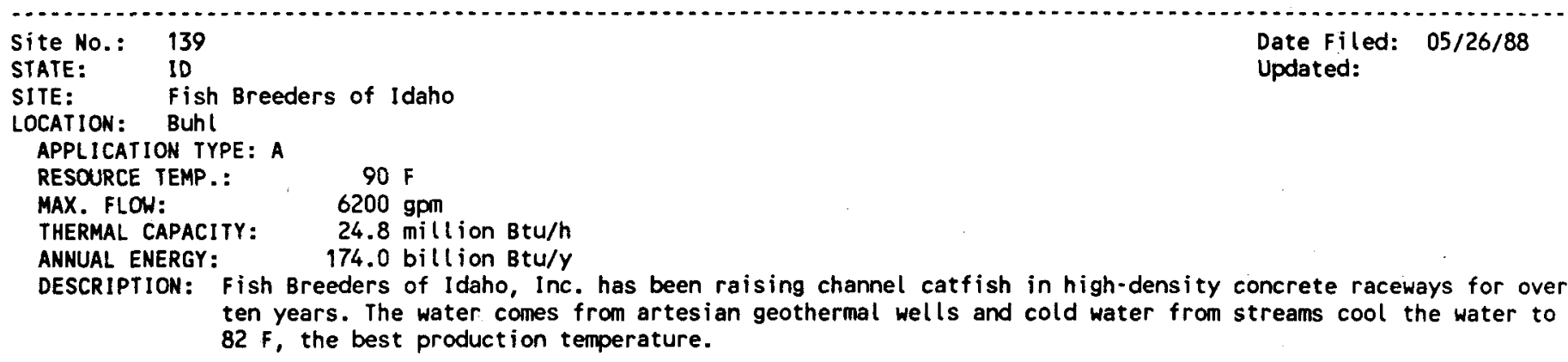

REFERENCE: Lund, 1987

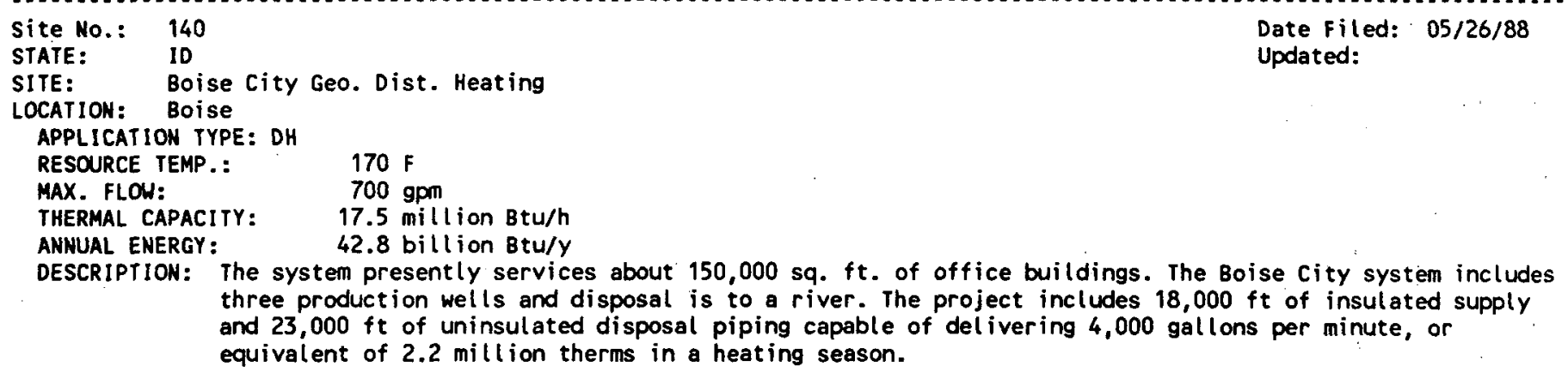


SITE: Idaho Capitol Mall

LOCATION: BOISE

APPLICATION TYPE: DH

RESOURCE TEMP.: $162 \mathrm{~F}$

MAX. FLOW: $\quad 750 \mathrm{gpm}$

THERMAL CAPACITY: $\quad 15.8 \mathrm{mill}$ ion Btu/h

ANNUAL ENERGY: $\quad 31.7$ billion Btu/Y

DESCRIPTION: The Idaho Capitol Mall consists of seven state office buildings, including the capitol.

REFERENCE: Lienau, 1984

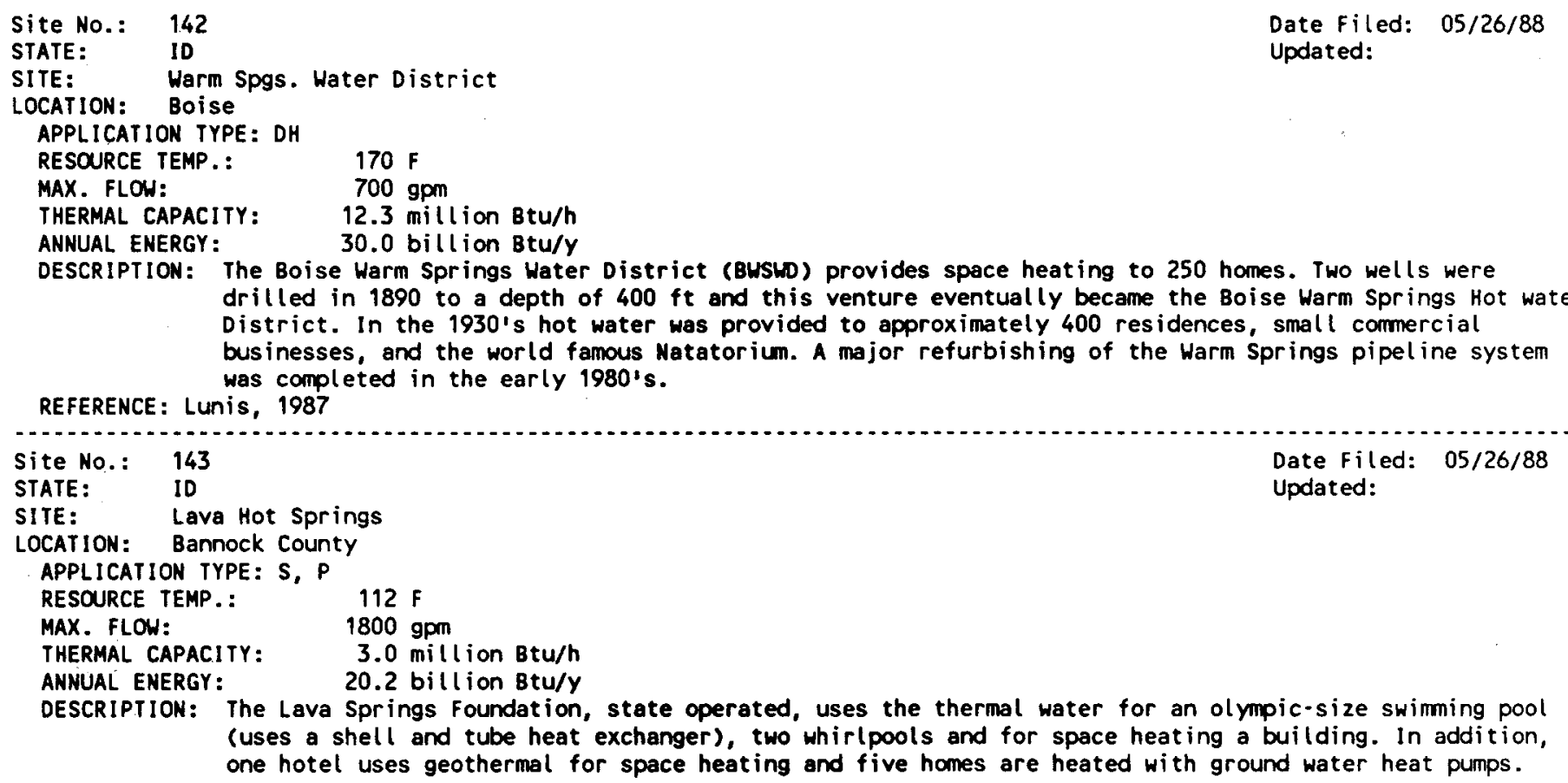

REFERENCE: Keller, 1988

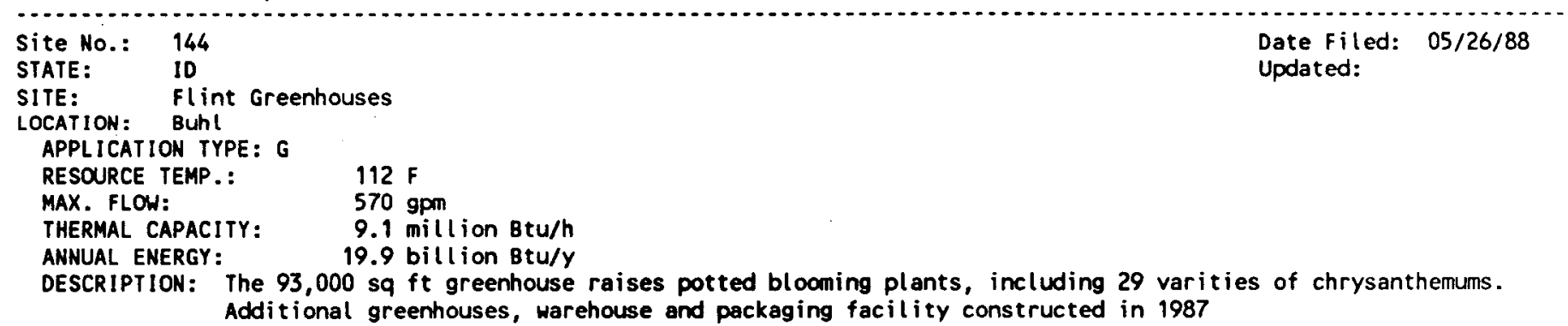

REFERENCE: Street, 1985; Culver, 1988

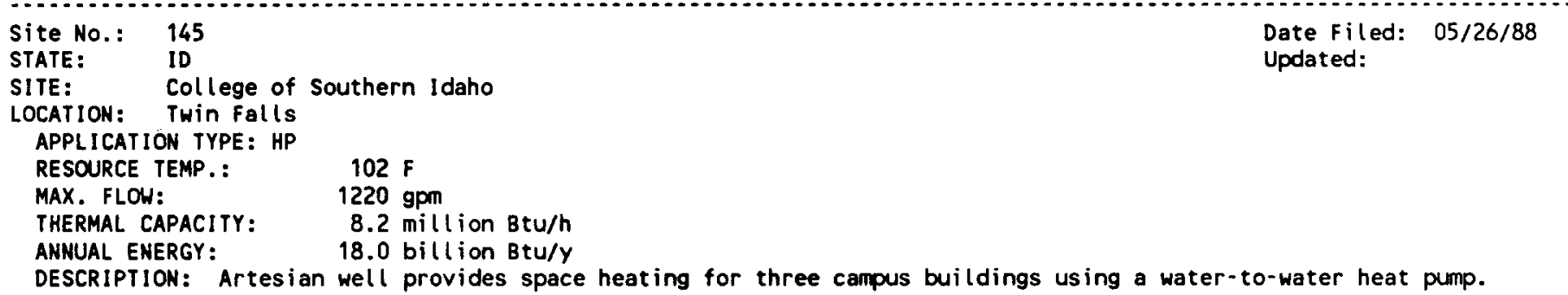




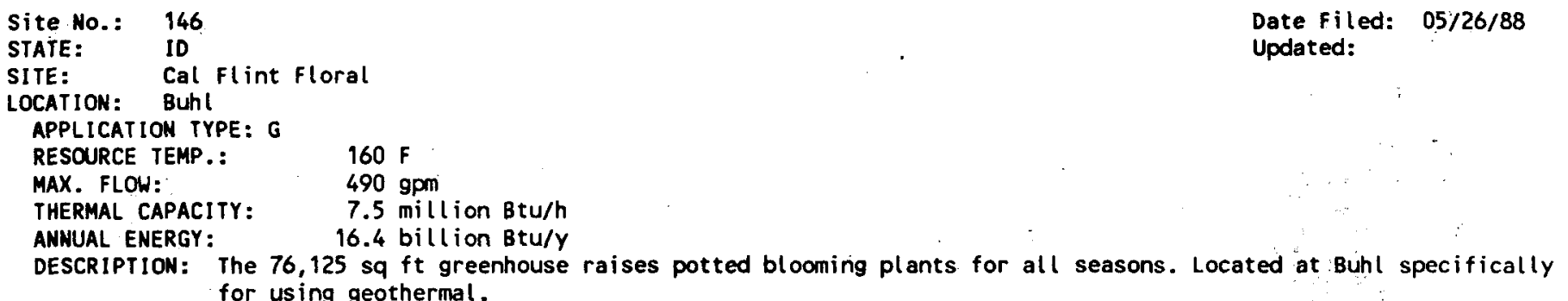
for using geothermal.

REFERENCE: Street, 1985

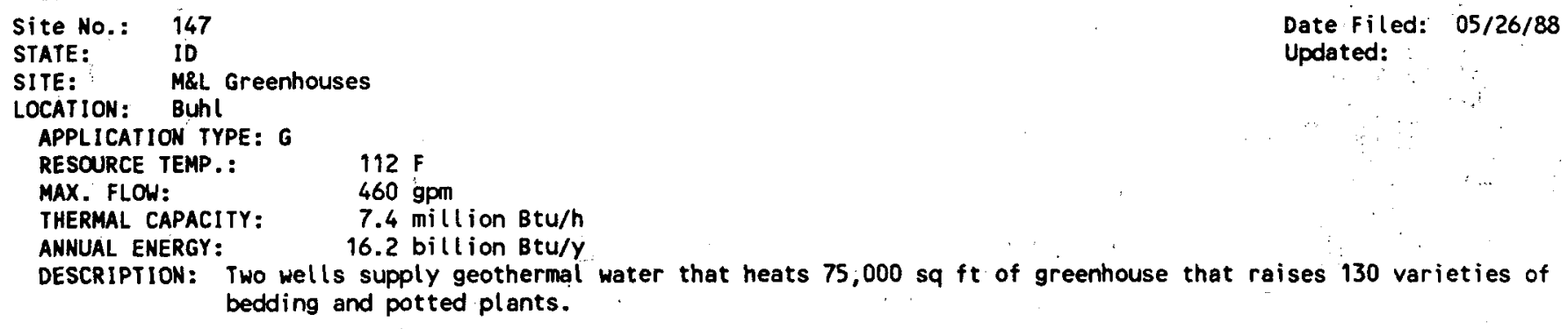

REFERENCE: Street, 1985

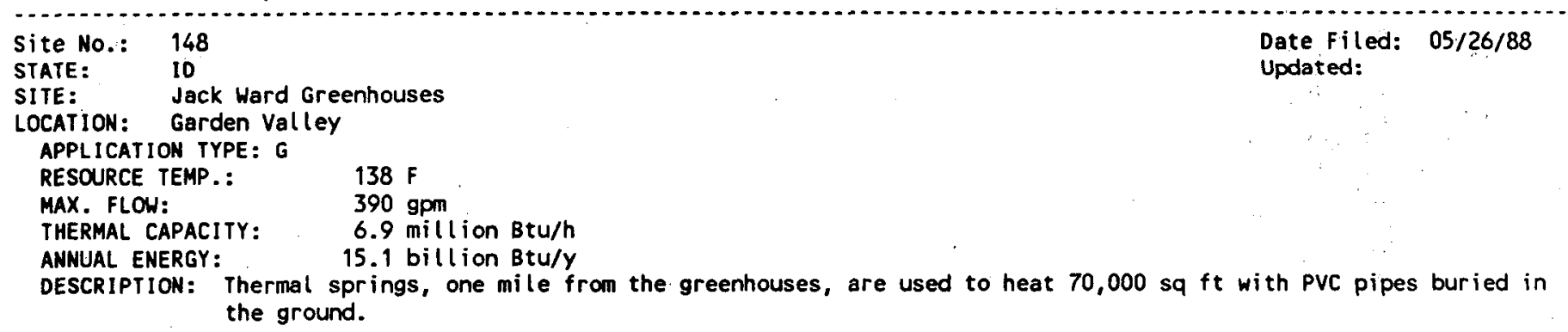

REFERENCE: Street, 1985

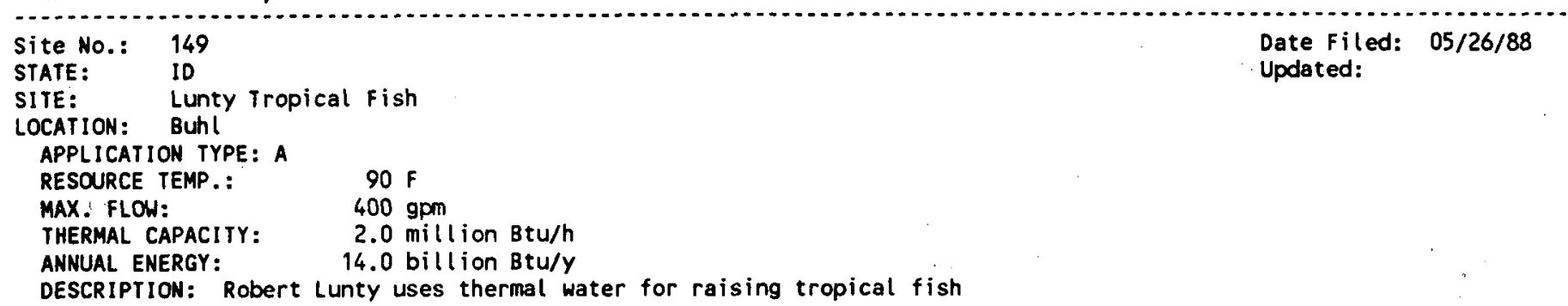

REFERENCE: Kenkermath, 1985

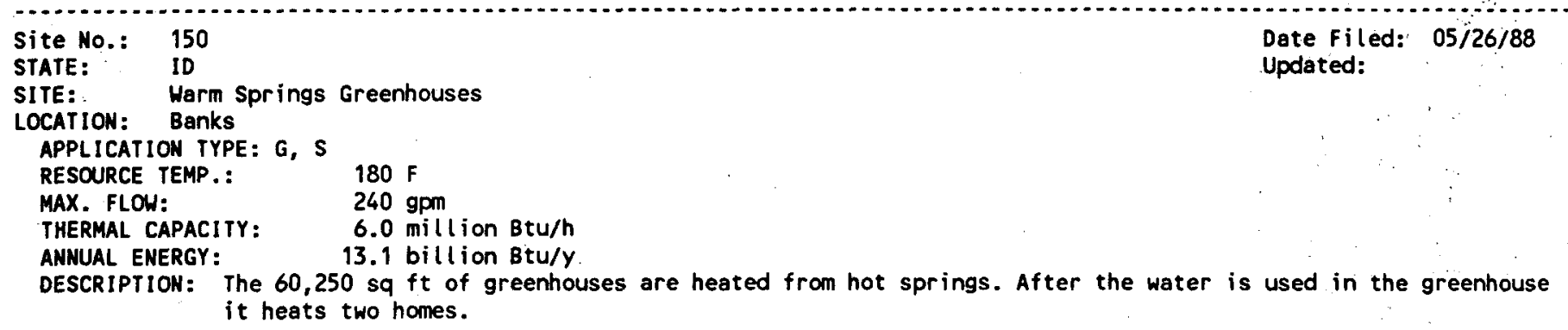


SITE: $\quad$ Fort Boise Veteran's Hospital

APPLICATION TYPE: S

RESOURCE TEMP.: $\quad 162 \mathrm{~F}$

MAX. FLOW: $\quad 300 \mathrm{gpm}$

THERMAL CAPACITY: $\quad 6.0 \mathrm{mill}$ (lion Btu/h

ANNUAL ENERGY: $\quad 12.1$ billion Btu/y

DESCRIPTION: Space and DHW heating are provided by two geothermal wells for central hospital and several service bul idings.

REFERENCE: Lunis, 1987

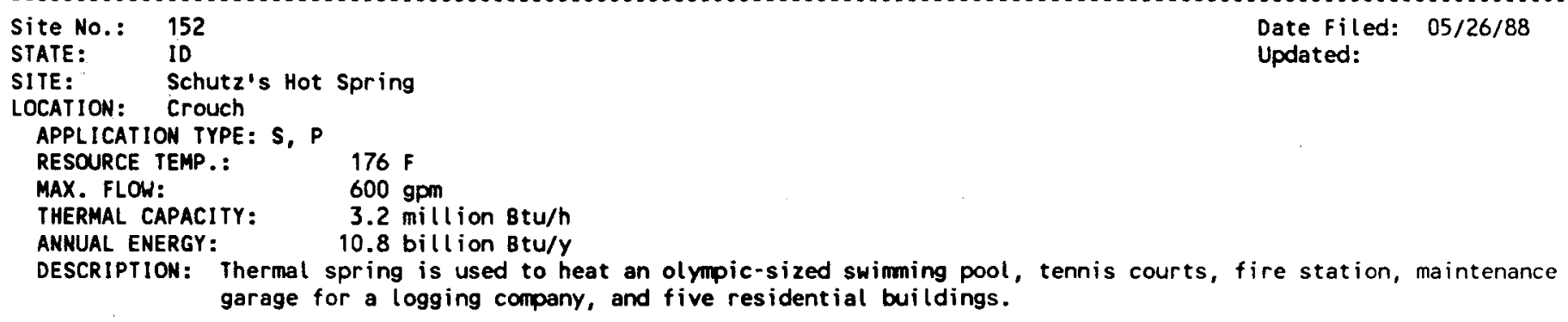

REFERENCE : McClain, 1979

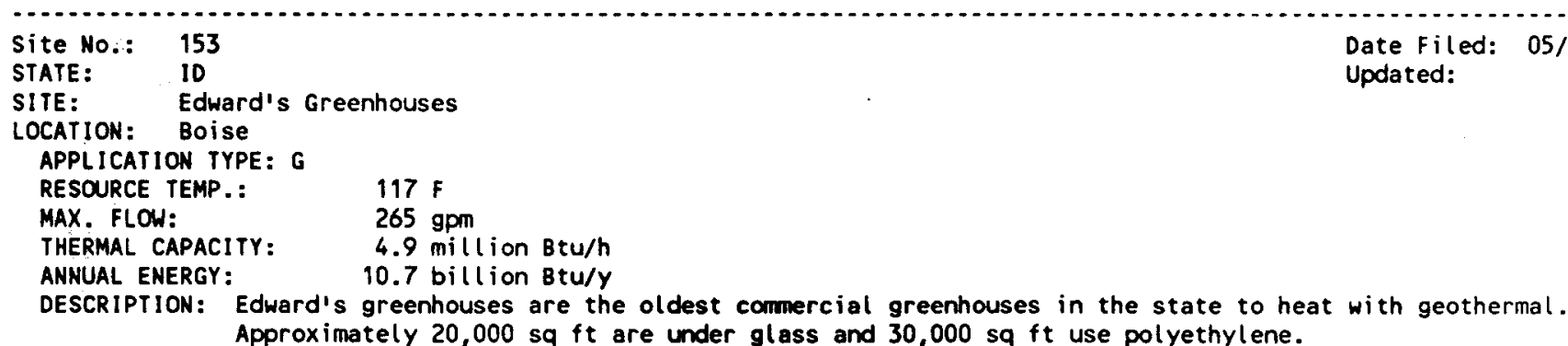

REFERENCE：Street, 1985

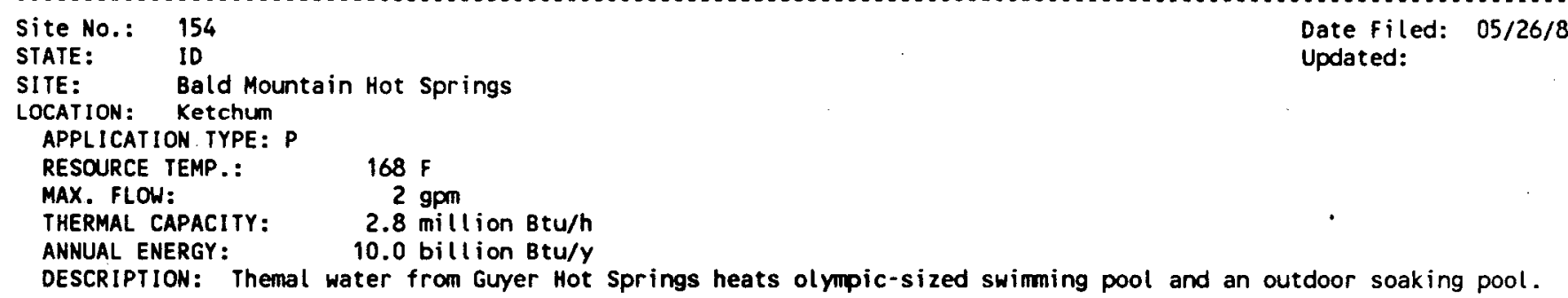

REFERENCE : Loam, 1980

Site NO.: $\quad 155$
STATE: $\quad$ ID
SITE: $\quad$ Crook's Greenhouse
LOCATION: Caksia County
APPLICATION TYPE: G
RESOURCE TEMP.: $\quad 194 \mathrm{~F}$
MAX. FLOW:
THERMAL CAPACITY: $\quad 40 \mathrm{gpm}$
ANNUAL ENERGY: $\quad 4.0 \mathrm{million} \mathrm{Btu/h}$
DESCRIPTION: Thermal water from a shallow well used to heat a greenhouse.


SITE: $\quad$ Sligar's Thousand Springs Res.

LOCATION: Hagerman

APPLICATION TYPE: P

RESOURCE TEMP.: $200 \mathrm{~F}$

MAX. FLOW: $\quad 140 \mathrm{gpm}$

THERMAL CAPACITY: $\quad 1.3 \mathrm{mill}$ ion Btu/h

ANHUAL ENERGY: $\quad 7.7$ billion Btu/Y

DESCRIPTION: Thermal springs heats large outdoor swimming pool and 17 jet pools.

REFERENCE: Loam, 1980

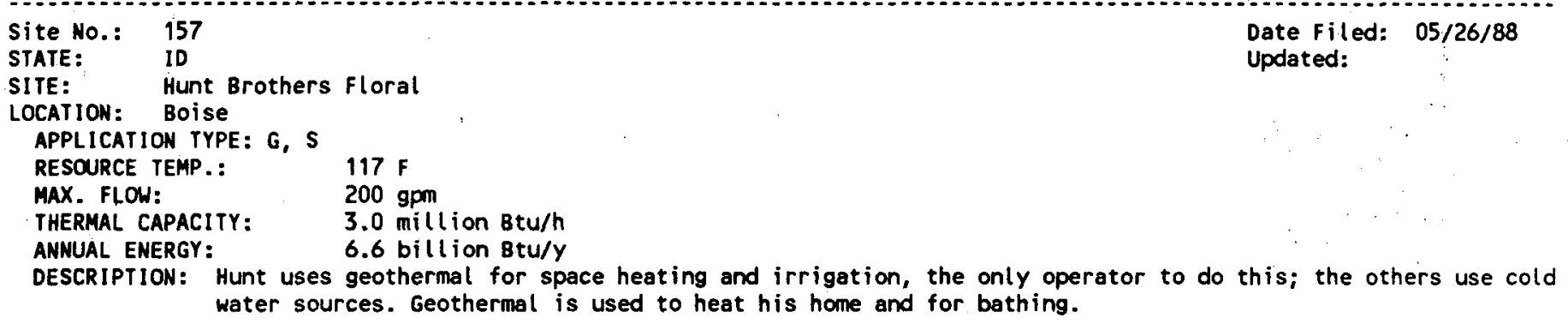

REFERENCE: Street, 1985

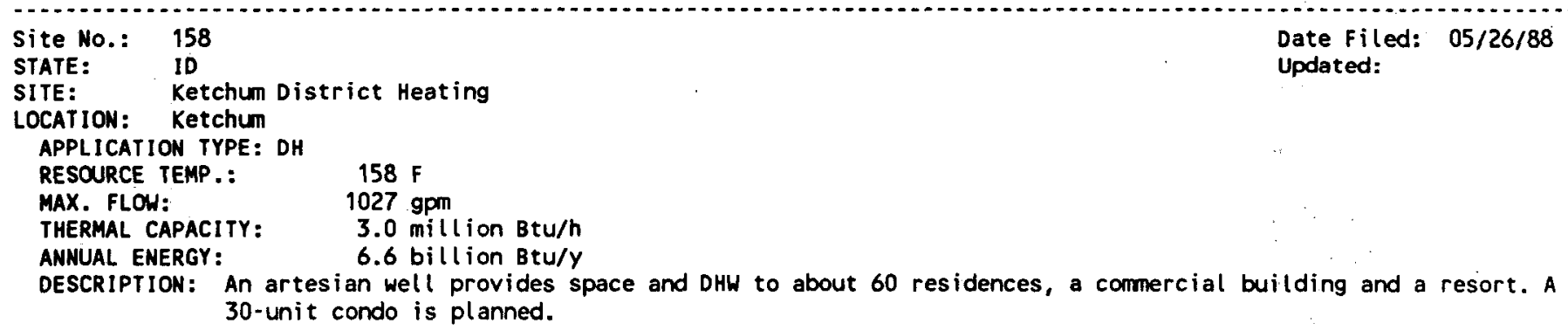

REFERENCE: Dell inger, 1982

Site No.: 159
STATE: $\quad$ ID
SITE: $\quad$ Jim's Hot Springs

REFERENCE : Loam, 1980

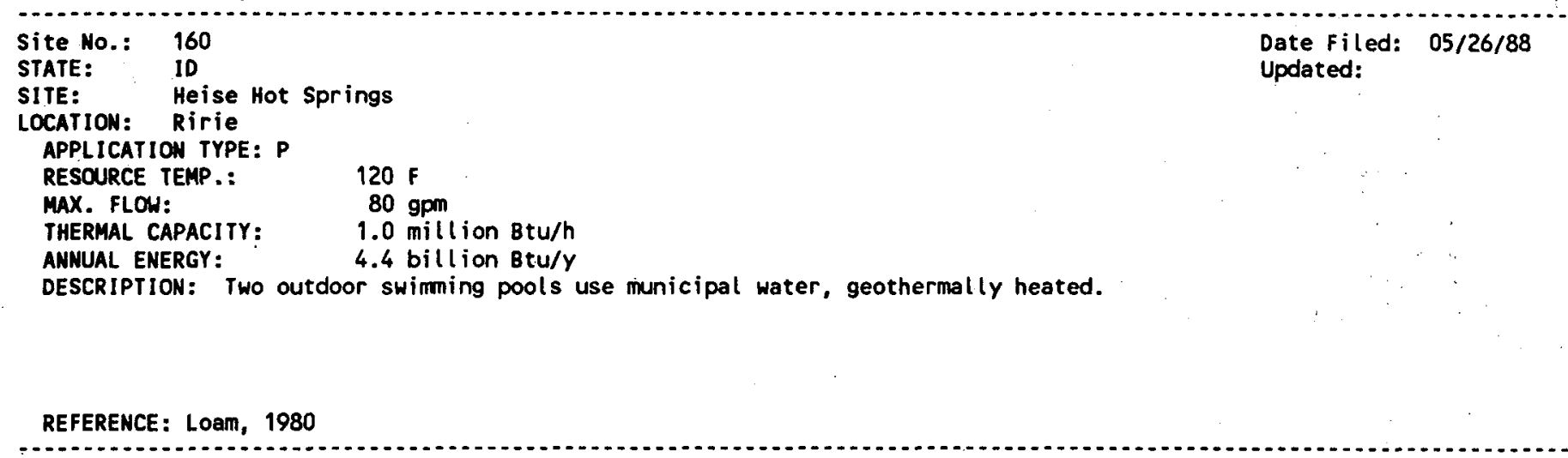


SITE: Bliss Greenhouse

LOCATION: BLISS

APPLICATION TYPE: G, S, A

RESOURCE TEMP.: $\quad 151 \mathrm{~F}$

MAX. FLOW: $\quad 60 \mathrm{gpm}$

THERMAL CAPACITY: $\quad 1.2 \mathrm{mill}$ ion $B t u / h$

ANNUAL ENERGY: $\quad 3.7$ billion Btu/y

DESCRIPTION: Geothermal heat is used in greenhouse, fish ponds, residence, personal swimming pool, and walkways exposed to snow.

REFERENCE: Loam, 1980

Site No.: 162
STATE: $\quad$ ID
SITE: $\quad$ Silver Creek Plunge
LOCATION: Garden Valley
APPLICATION TYPE: P
RESOURCE TEMP.: $\quad 101 \mathrm{~F}$
MAX. FLOW:
THERMAL CAPACITY: $\quad 0.9 \mathrm{mill}$ ion Btu/h
ANNUAL ENERGY:
DESCRIPTION: Thermal spring heats outdoor swimming pool.

REFERENCE : LOam, 1980

$\begin{array}{lll}\text { Site No.: } & 163 & \text { Date Filed: } \\ \text { STATE: } & \text { ID } & \text { O5/26/88 } \\ \text { SITE: } & \text { Haven Lodge } & \text { Updated: }\end{array}$

Lodge

APPLICATION TYPE: P

RESOURCE TEMP.: $\quad 148 \mathrm{~F}$

MAX. FLOW: $\quad 50 \mathrm{gpm}$

THERMAL CAPACITY: $\quad 0.5 \mathrm{mill}$ ion Btu/h

ANNUAL ENERGY: $\quad 3.5$ billion Btu/Y

DESCRIPTION: Thermal spring heats outdoor swimming pool.

REFERENCE: LOam, 1980
Site NO.: $\quad 164$
STATE: $\quad$ ID
SITE: $\quad$ Warm Springs Resort
LOCATION: Idaho City
APPLICATION TYPE: P
RESOURCE TEMP.:
MAX. FLOW:
THERMAL CAPACITY: $\quad 110 \mathrm{~F}$
ANNUAL ENERGY: $\quad 0.9 \mathrm{million}$ Btu/h
DESCRIPTION: Thermal spring heats rural plunge.

REFERENCE: Loam, 1980

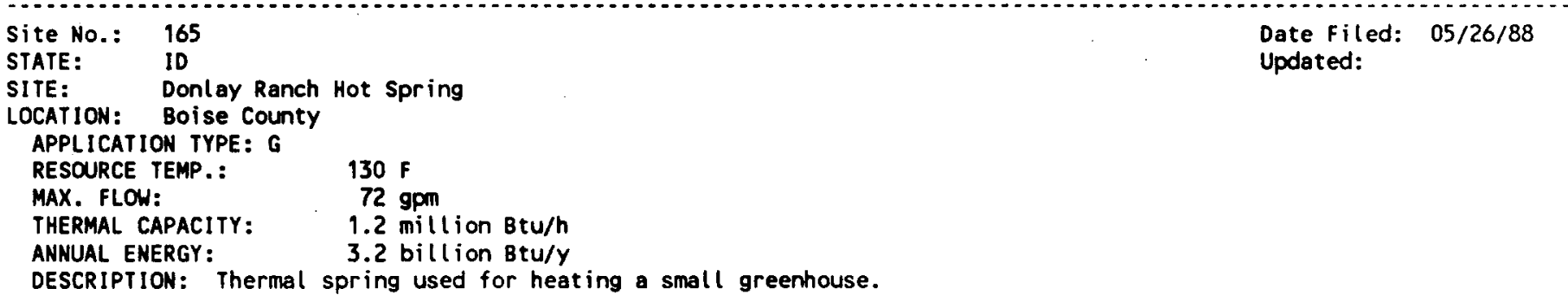


SITE: Miracle Hot Springs

LOCATION: BUhl

APPLICATION TYPE: S, P

RESOURCE TEMP.: $139 \mathrm{~F}$

MAX. FLOW: $\quad 50 \mathrm{gpm}$

THERMAL CAPACITY: $\quad 0.7 \mathrm{mill}$ ion $\mathrm{Btu} / \mathrm{h}$

ANNUAL ENERGY: $\quad 2.9 \mathrm{bill}$ : $\quad$ Btu/Y

DESCRIPTION: Thermal spring heats outdoor swimming pool and 15 enclosed soaking pools. All buildings and dressing rooms are heated with geothermal.

REFERENCE: LOam, 1980

\begin{tabular}{|c|c|c|c|}
\hline $\begin{array}{ll}\text { Site No.: } & 167 \\
\text { STATE: } & \text { ID } \\
\text { SITE: } & \text { Downatta Hot } \\
\text { LOCATION: } & \text { DOWney } \\
\text { APPLICATION TYPE: P } \\
\text { RESOURCE TEMP.: } \\
\text { MAX.: FLOW: } \\
\text { THERMAL CAPACITY: } \\
\text { ANNUAL ENERGY: }\end{array}$ & $\begin{array}{l}112 \mathrm{~F} \\
80 \mathrm{gpm} \\
0.6 \mathrm{milli} \text { on } \mathrm{Btu} / \mathrm{h} \\
2.5 \mathrm{bill} \text { ion } 8 t u / y\end{array}$ & $\begin{array}{l}\text { Date Filed: } \\
\text { Updated: } \\
\qquad\end{array}$ & $\begin{array}{c}05 / 26 / 88 \\
\ddots\end{array}$ \\
\hline
\end{tabular}

REFERENCE : LOam, 1980

Site NO:: 168
STATE:
SITE:
ID
LOCATION: Buhl
APPLICATION TYPE: S, P
RESOURCE TEMP.: $\quad 131 \mathrm{~F}$
MAX. FLOW:
THERMAL CAPACITY: $\quad 00 \mathrm{gpm}$
ANNUAL ENERGY: $\quad 0.8 \mathrm{million}$ Btu/h
DESCRIPTION: Thermal spring heats outdoor swimming pool, locker rooms, and several buidings.

REFERENCE : LOam, 1980

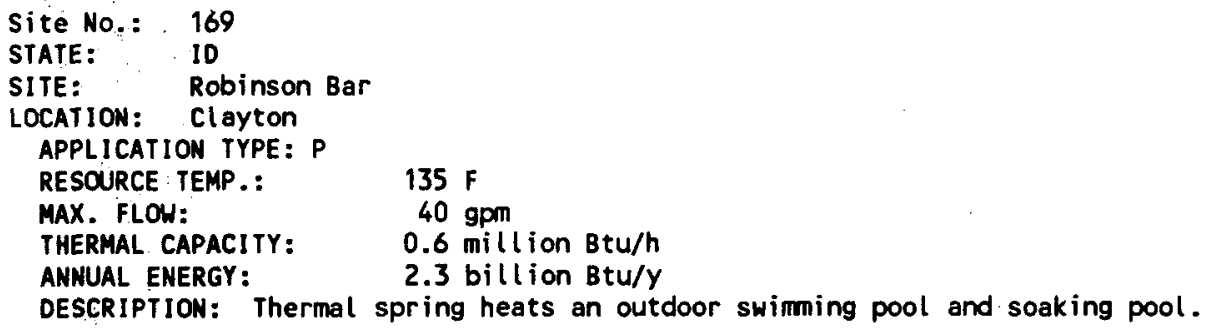

Date filed: $05 / 26 / 88$ Updated:

REFERENCE: LOam, 1980

Site No.: 170
STATE: $\quad$ ID
SITE: $\quad$ Challis Hot Springs
LOCATION: Challis
APPLICATION TYPE: P
RESOURCE TEMP.: $\quad 127 \mathrm{~F}$
MAX. FLOW:
THERMAL CAPACITY: $\quad 40 \mathrm{gpm}$
ANMUAL ENERGY: $\quad 0.5 \mathrm{mill}$ ion Btu/h
DESCRIPTION: Thermal spring heats swiming pool.


SITE: Indian Springs Natatorium

LOCATION: American Falls

APPLICATION TYPE: P

RESOURCE TEMP : $\quad 90$

MAX. FLOW: $\quad 120 \mathrm{gpm}$

THERMAL CAPACITY: $\quad 0.6 \mathrm{mill}$ ion Btu/h

ANNUAL ENERGY: $\quad 2.1 \mathrm{bill}$ ion Btu/Y

DESCRIPIION: Thermal spring heats outdoor swimming pool.

REFERENCE: Loam, 1988

Site No.: $\quad 172$
STATE: $\quad$ ID
SITE: $\quad$ Green Canyon Hot Springs
LOCATION: Newdale
APPLICATION TYPE: G, P $\quad 118 \mathrm{~F}$
RESOURCE TEMP.: $\quad 80 \mathrm{gpm}$
MAX. FLOW:
THERMAL CAPACITY: $\quad 0.6 \mathrm{mill}$ ion Btu/h
ANNUAL ENERGY: $\quad 2.0 \mathrm{billion}$ Btu/y
DESCRIPTION: Thermal spring heats indoor swimming pool. Geothermal greenhouse produce hydroponic tomatoes and
cucumbers.

REFERENCE: Loam, 1980

Site No.: 173
STATE: $\quad$ ID
SITE: $\quad$ Bear Lake Hot Springs

REFERENCE: Loam, 1980

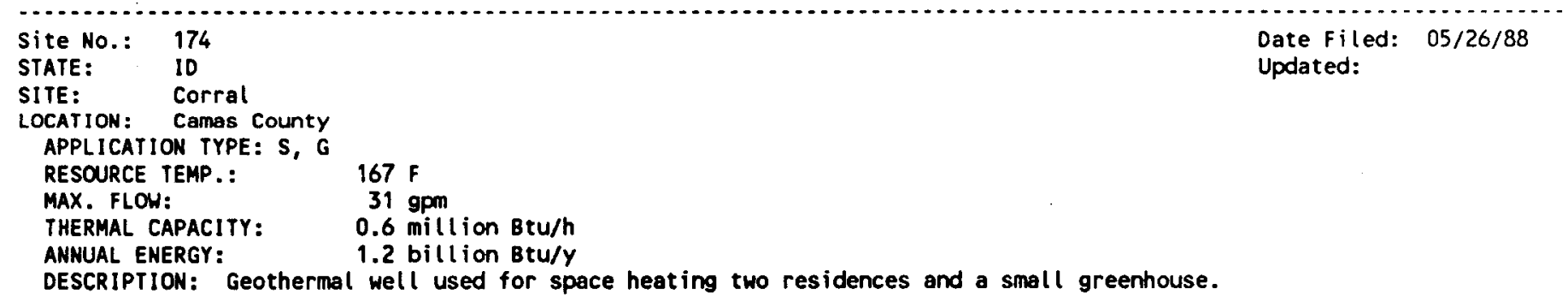

REFERENCE: Lienau, 1986

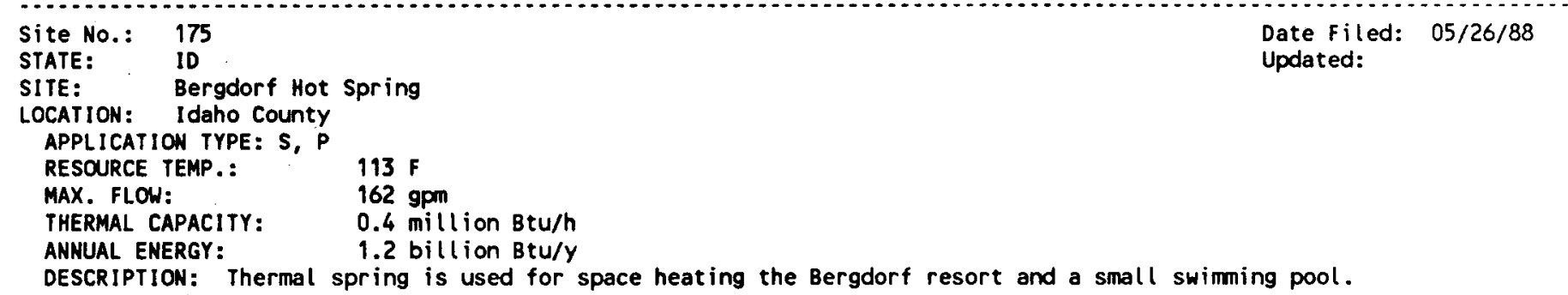


LOCATION: ALTO

APPLICATION TYPE: S

RESOURCE TEMP.:

MAX. FLOW:

$93 \mathrm{~F}$

THERMAL CAPACITY:

$80 \mathrm{gpm}$

ANNUAL ENERGY:

$0.4 \mathrm{mill}$ ion Btu/h

DESCRIPIION: Geothermal well provides space heat to a $13,800 \mathrm{sq}$. $\mathrm{ft}$. church and for sidewalk snow melt.

REFERENCE: Kenkermath, 1985

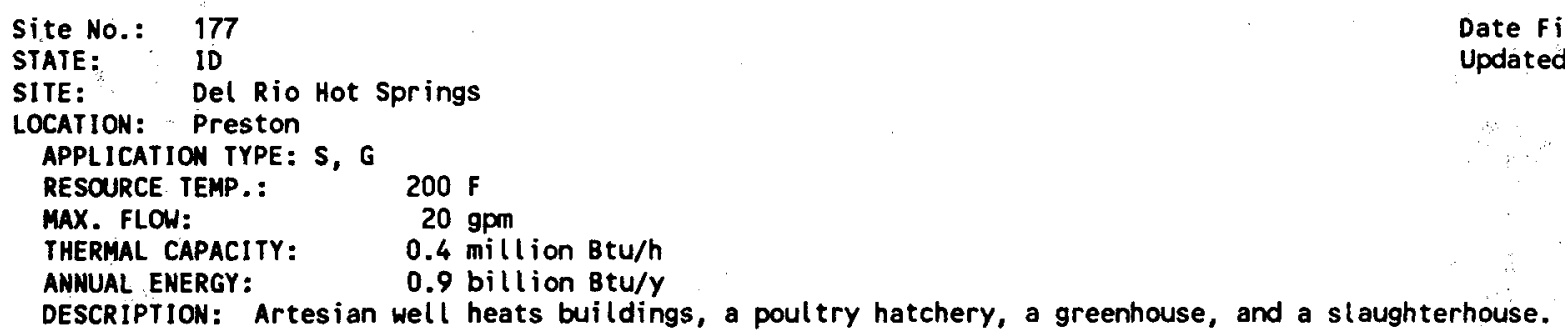

REFERENCE : Loam, 1980

Site NO.: 178
STATE:
SITE: $\quad$ Riggins Hot Springs
LOCATION: Idaho County
APPLICATION TYPE: G, S
RESOURCE TEMP.: $\quad 113 \mathrm{~F}$
MAX. FLOW:
THERMAL CAPACITY: $\quad 50 \mathrm{gpm}$
ANNUAL ENERGY: $\quad 0.4 \mathrm{mill}$ Updated:
DESCRIPTION: Thermal spring used to heat a small resort and commercial greenhouse

REFERENCE: McClain, 1979

Site No.: 179

STATE: ID

SITE: $\quad$ Express Farms

LOCATION: Marsing

APPLICATION TYPE: $G$

RESOURCE TEMP.:

MAX. FLOW:

THERMAL CAPACITY:

ANNUAL ENERGY:

$47 \mathrm{gpm}$

$0.4 \mathrm{mill}$ ion Btu/h

0.8 billion Btu/y

DESCRIPTION: The $3,600 \mathrm{sq} \mathrm{ft}$ greenhouse is used to raise tomatoes
Date Filed: $05 / 26 / 88$ Updated:

REFERENCE: Street, 1985

Site NO.: 180
STATE: $\quad$ ID
SITE: $\quad$ Heiser Hot Springs


SITE: Givens Hot Springs

LOCATION: Owyhee County

APPLICATION TYPE: $P$

RESOURCE TEMP.: $120 \mathrm{~F}$

MAX. FLOW: $\quad 650 \mathrm{gpm}$

THERMAL CAPACITY: $\quad 0.2 \mathrm{million}$ Btu/h

ANMUAL ENERGY: $\quad 0.5 \mathrm{bill}$ ion Btu/Y

DESCRIPIION: A small swiming pool is heated.

REFERENCE: MCClain, 1979

$\begin{array}{ll}\text { Site No.: } 182 & \\ \text { STATE: } \quad \text { ID } & \\ \text { SITE: } \quad \text { Stanley Dist. Htg. (proposed) } \\ \text { LOCATION: Stanley } \\ \text { APPLICATION TYPE: DH } \\ \text { RESOURCE TEMP.: } & 140 \mathrm{~F} \\ \text { MAX. FLON: } & 290 \mathrm{gpm} \\ \text { THERMAL CAPACITY: } & 0.0 \mathrm{mill} \\ \text { ANNUAL ENERGY: } & 0.0 \mathrm{bill} \text { btuon } \mathrm{Btu} / \mathrm{h}\end{array}$

DESCRIPTION: The district heating system could consist of 35 residences, 16 businesses, 5 motels, and one school.

REFERENCE: Dellinger, 1981

Site NO.: 183
STATE: $\quad$ ID
SITE: $\quad$ Twin Springs Resort
LOCATION: BOISe
APPLICATION TYPE: S, P
RESOURCE TEMP.: $\quad 180 \mathrm{~F}$
MAX. FLOW:
THERMAL CAPACITY: gpm
ANNUAL ENERGY: $\quad$ million Btu/h
DESCRIPTION: Thermal water heats an enclosed sosking pool, all buildings, ond some of the water piped from the
$\quad$ springs turns a Pelton Water Wheel to generate electricity used at the resort.

REFERENCE : Loam, 1980

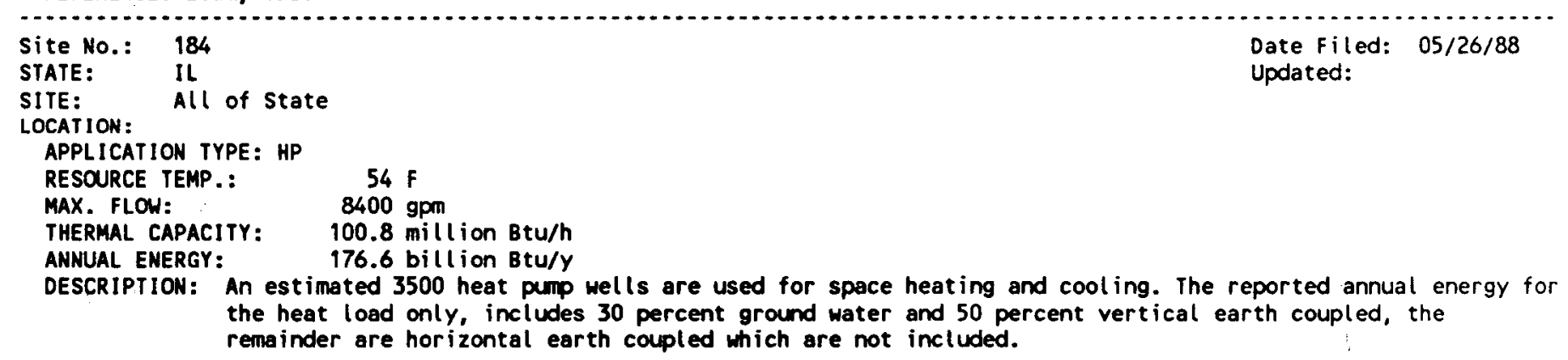

REFERENCE: MCCray, 1988; Ellis, 1988

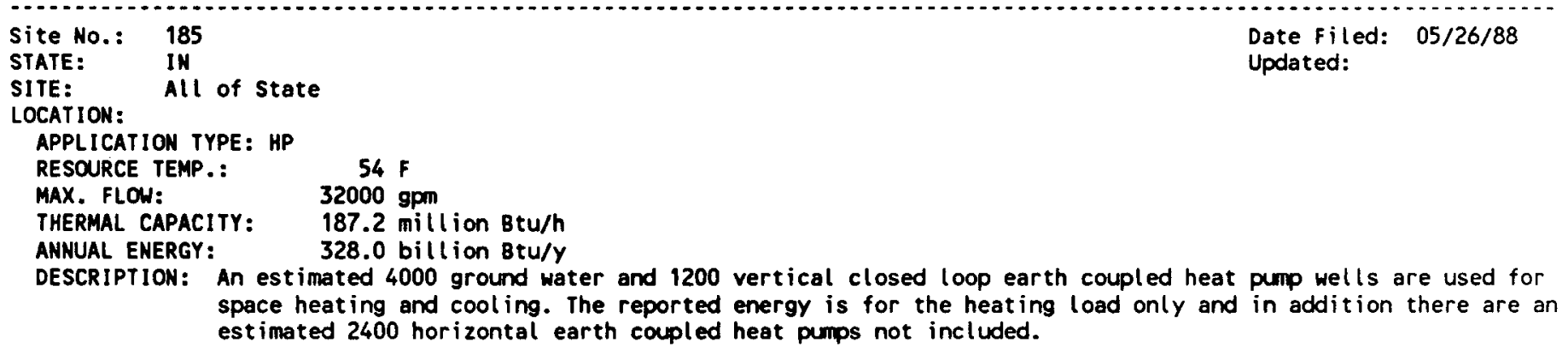




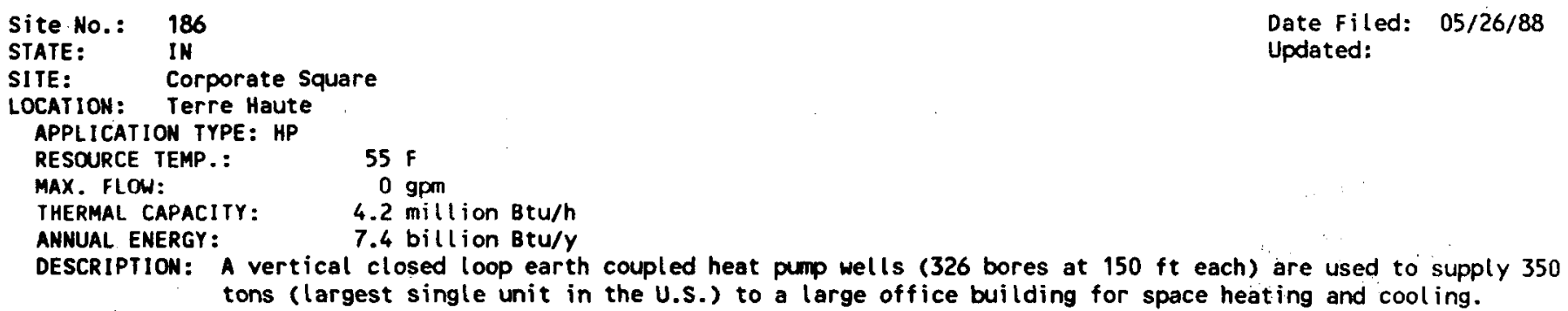

REFERENCE: Ellis, 1988

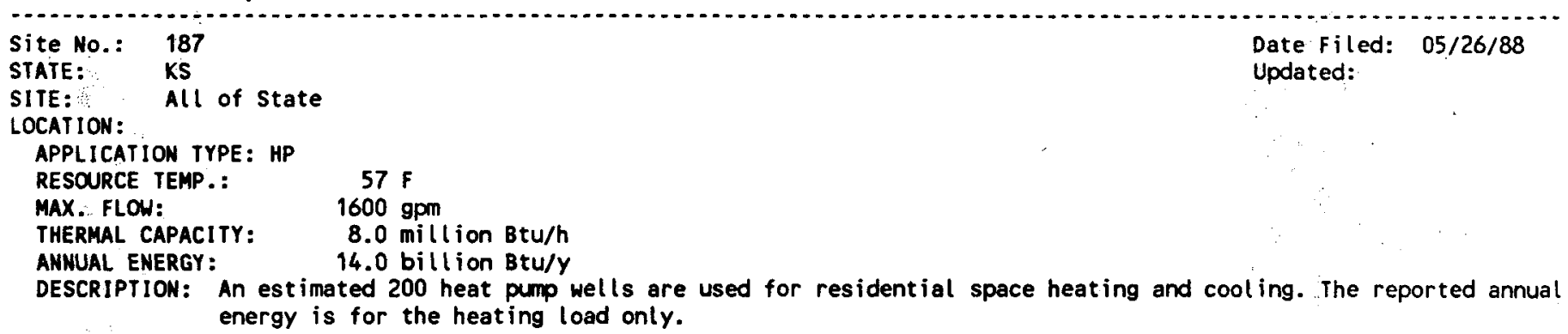

REFERENCE: Norton, 1988

Site NO.: 188
STATE: $\quad$ KS
SITE: $:$ Elementary Schools (3)

REFERENCE: Norton, 1988

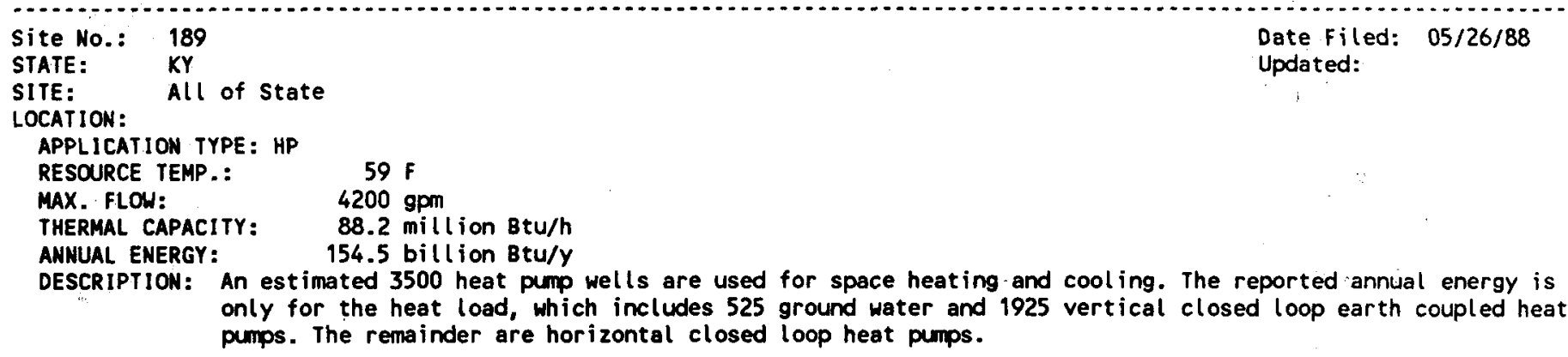

REFERENCE : Ell is, 1988

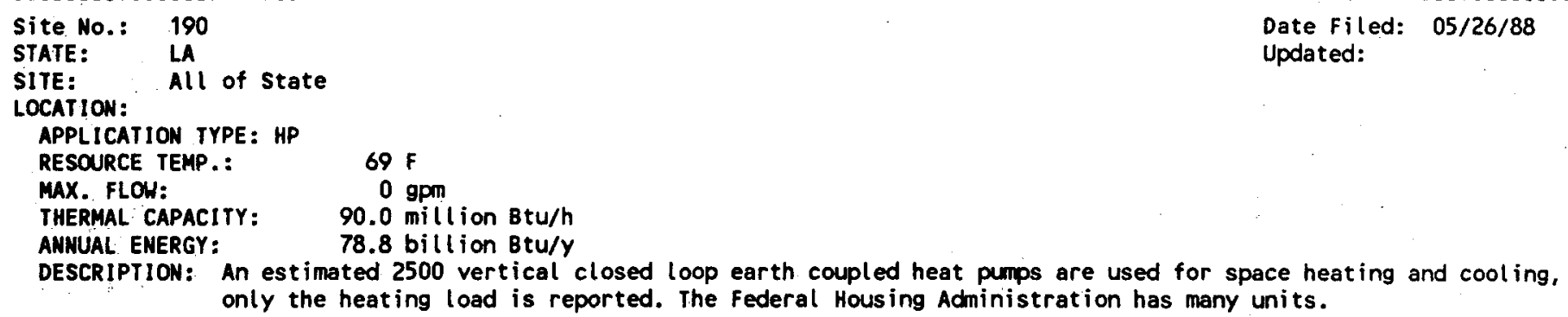


OCATION: BOStOO

APPLICATION TYPE: HP

RESOURCE TEMP.

THERMAL CAPACITY:

$0.4 \mathrm{mill}$ ion Btu/h

DESCRIPTION: Ground water heat pump used for space heating

REFERENCE: Mcafee, 1988

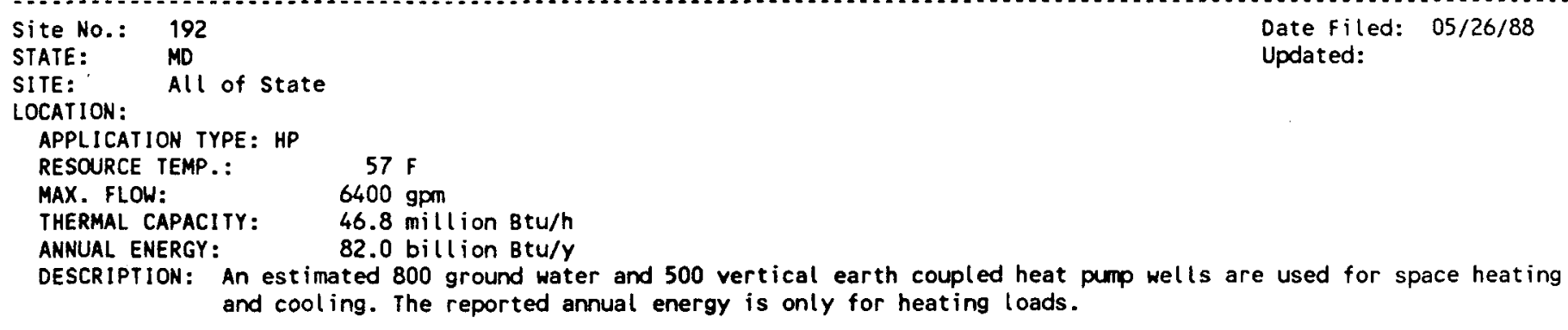

REFERENCE: MCCray, 1988; Ellis; 1988

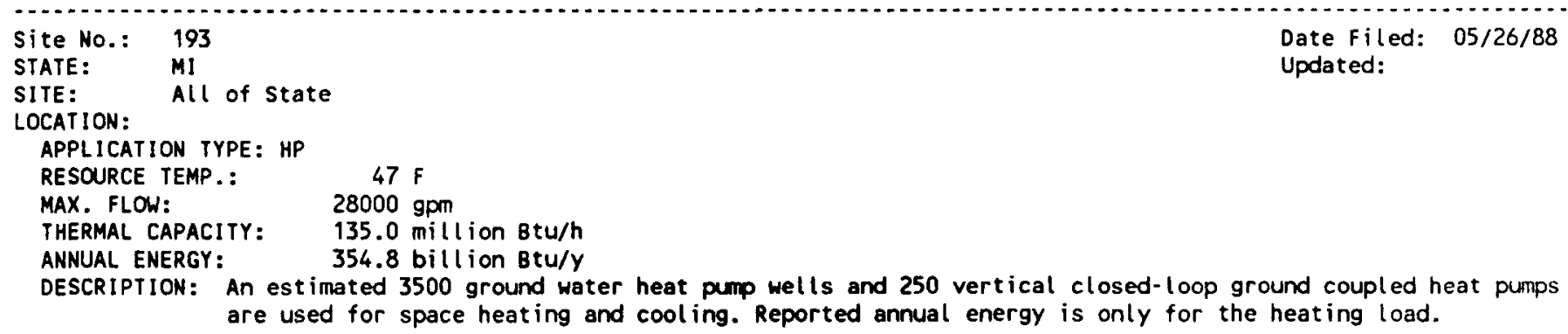

REFERENCE : Ell is, 1988

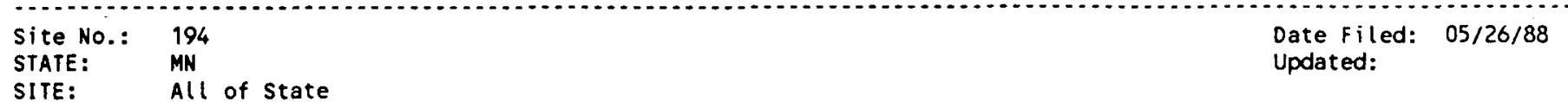

SITE: $\quad$ All of State

LOCATION:

APPLICATION TYPE: HP

RESOURCE TEMP.: $\quad 45 \mathrm{~F}$

MAX. FLOW: $\quad 6800 \mathrm{gpm}$

THERIMAL CAPACITY: $\quad 36.0$ million Btu/h

ANNUAL ENERGY: $\quad 94.6$ billion Btu/Y

DESCRIPIION: An estimated 1000 heat pump wells are used for space heating and cooling. These represent 85 percent ground water and 15 percent vertical closed-loop ground coupled. The reported annual energy is only for the heating load.

REFERENCE: Ellis, 1988

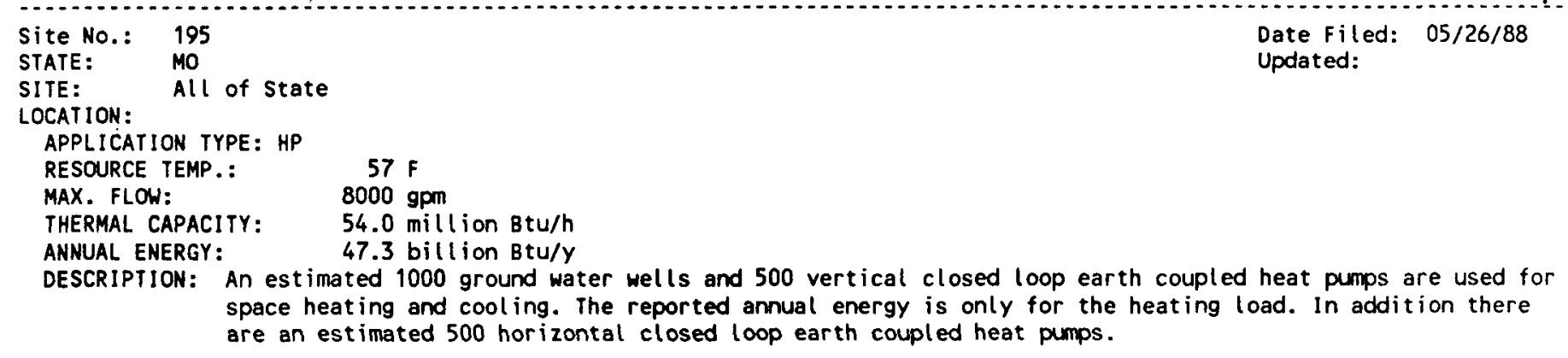


LOCATION :

APPLICATION TYPE: HP

RESOURCE TEMP.: $\quad 67 \mathrm{~F}$

MAX. FLOW: $\quad 800 \mathrm{gpm}$

THERMAL CAPACITY: $\quad 4.0 \mathrm{mill}$ ion Btu/h

ANNUAL ENERGY: $\quad 3.5$ billion $8 t u / Y$

DESCRIPTION: An estimated 100 ground water heat pump wells are used for space heating and cooling.

REFERENCE: Schult2, 1988

Site NO.: 197
STATE: $\quad$ MS
SITE: $\quad$ Mississippi Power Co.
LOCATION: mainly southern part
APPLICATION TYPE: HP
RESOURCE TEMP.: $\quad 69 \mathrm{~F}$
MAX. FLOW: $\quad 0 \mathrm{gPm}$
THERMAL CAPACITY: $\quad 1.1 \mathrm{mill}$ ion Btu/h
ANMUAL ENERGY: $\quad 0.9 \mathrm{bill}$ ion Btu/Y
DESCRIPTION: An estimated 89 tons for Biloxi, Poplarville, Taylorsville, and Bay St. Louis are installed for space
$\quad$ heating and cooling using vertical earth coupled heat pump wells.

REFERENCE : Patton, 1988

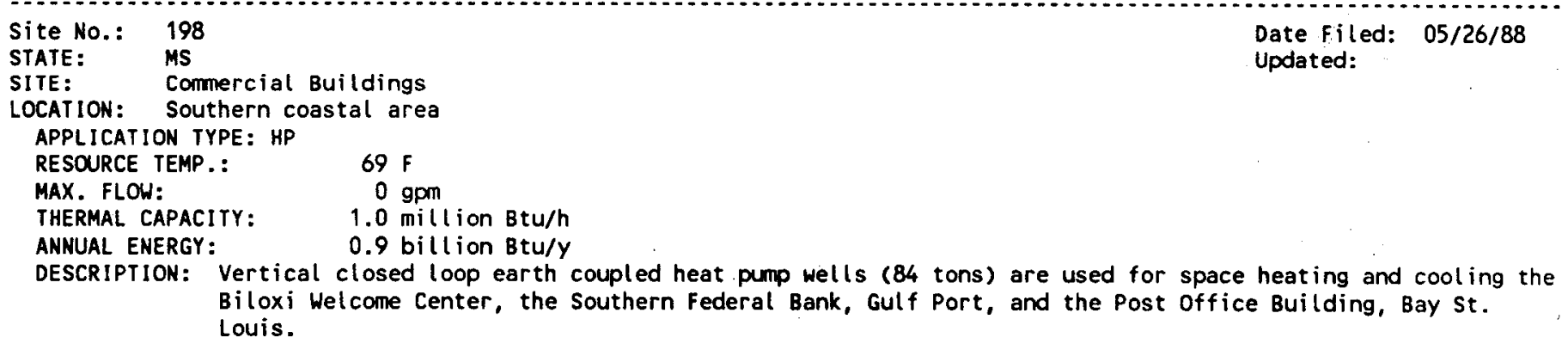

REFERENCE : Patton, 1988

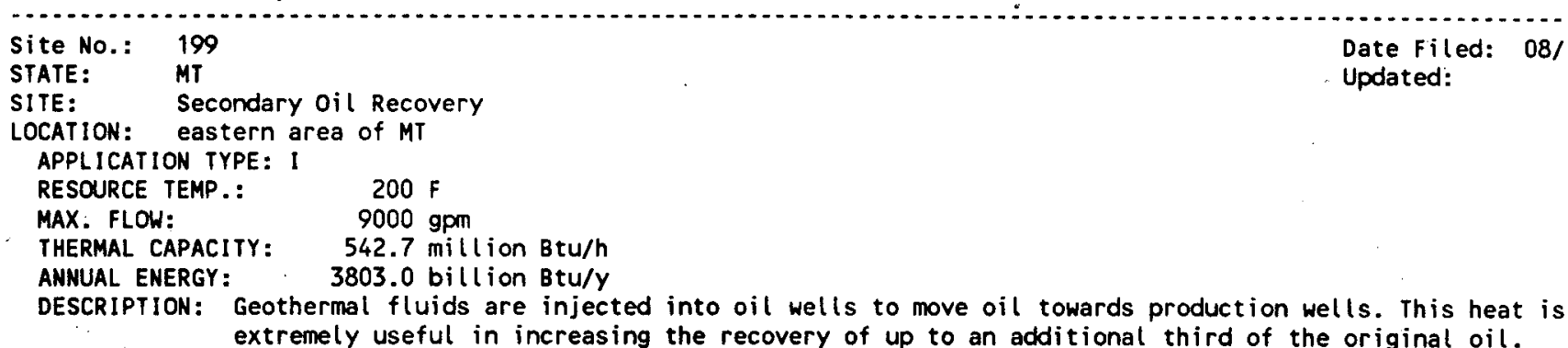

REFERENCE: Reed, 1988

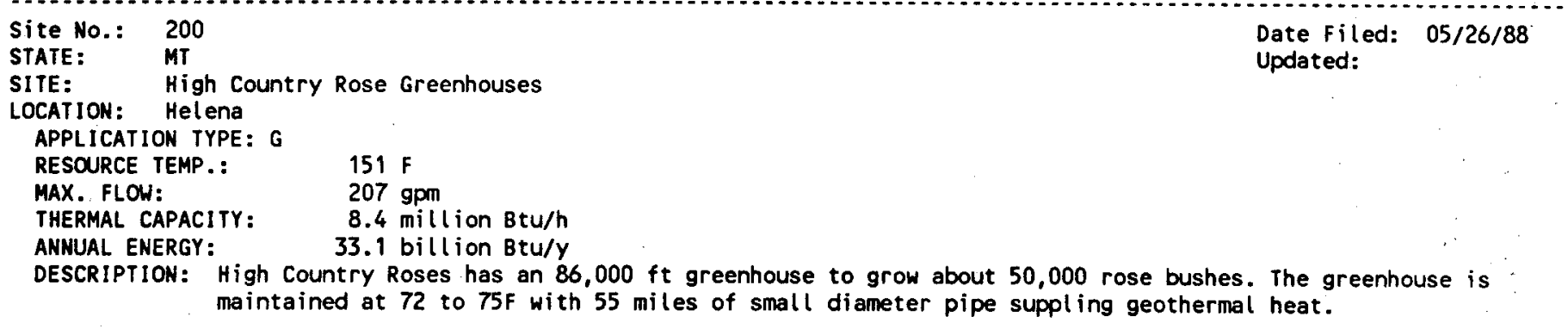




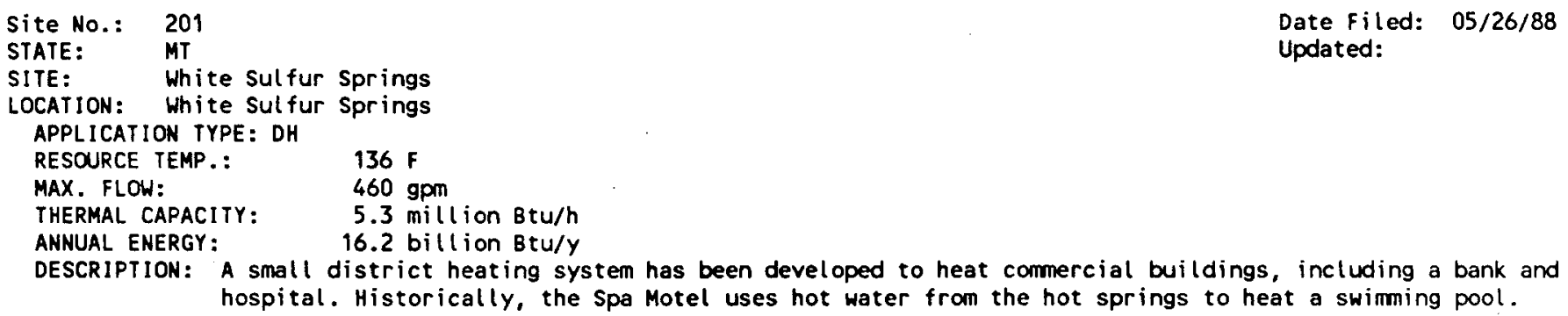

REFERENCE: Sonderigger, 1981
Site No.: 202

STATE: MT

SITE: . Warm Springs State Hospital

LOCATION: Harm Springs

APPLICATION TYPE: S

RESOURCE TEMP.: $\quad 154 \mathrm{~F}$

MAX. FLOW: 90 gpm

THERMAL CAPACITY: $\quad 1.9 \mathrm{mill}$ ion Btu/h

ANNUAL ENERGY: $\quad 14.6$ billion Btu/Y

DESCRIPIION: A moderate temperature (154F) resource provides space and DHW to the hospital. A USDOE PON project.
Date Filed: $05 / 26 / 88$ Updated:

REFERENCE : Lunis, 1986

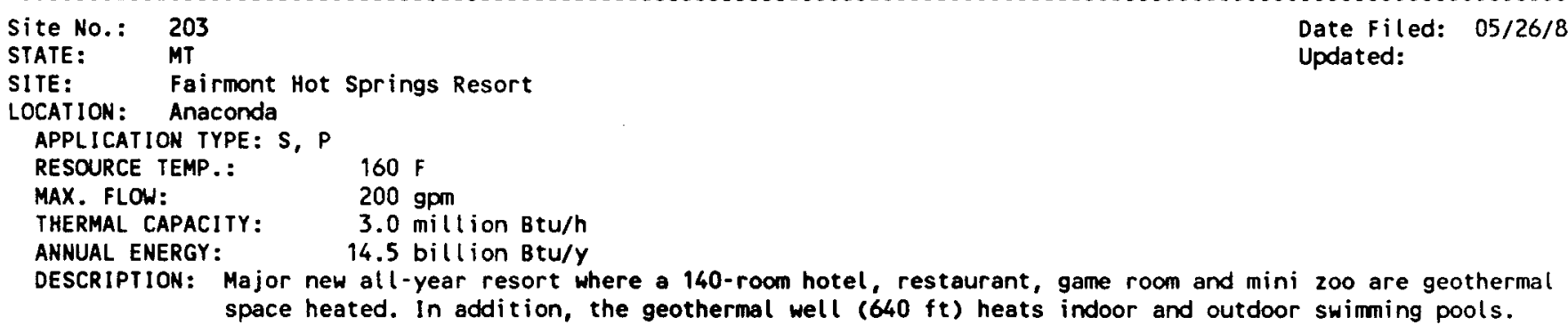

REFERENCE : Green, 1988

Site NO.: 204
STATE: MT
SITE:
LOCATION: MisSOUla County
APPLICATION TYPE: S, P
RESOURCE TEMP.: $\quad 111 \mathrm{~F}$
MAX. FLOW:
THERMAL CAPACITY: $\quad 180 \mathrm{gpm}$
ANNUAL ENERGY: $\quad 1.8 \mathrm{million}$ Btu/h
DESCRIPTION: Thermal spring provides space heating to resort facilities, outdoor swimming and indoor soaking pools
and is open alt year.

REFERENCE : LOam, 1980

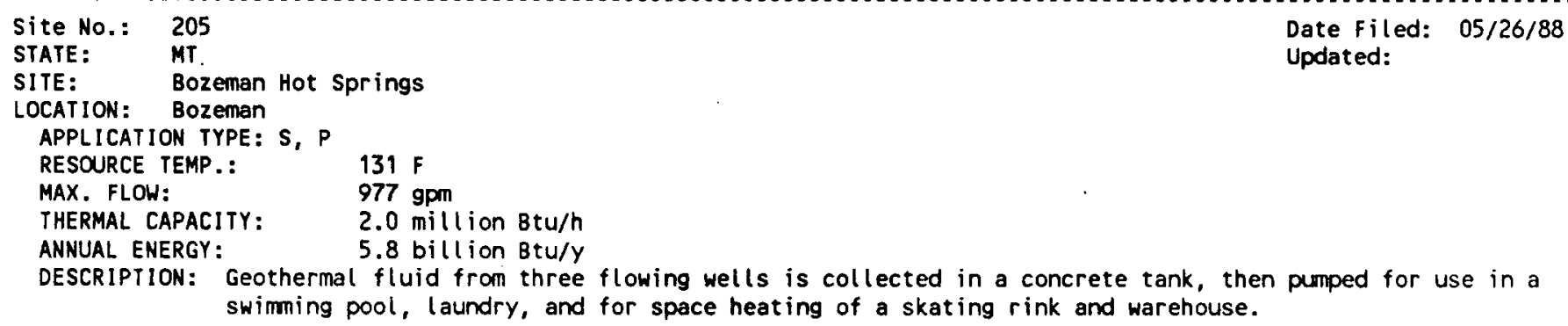


SITE: $\quad$ Broadwater Athletic Club \& HS

LOCATION: Helena

APPLICATION TYPE: S, P

RESOURCE TEMP.: $\quad 153 \mathrm{~F}$

MAX. FLOW: $\quad 100 \mathrm{gpm}$

THERMAL CAPACITY: $\quad 1.4 \mathrm{mill}$ ion Btu/h

ANNUAL ENERGY: $\quad 5.6$ billion Btu/y

DESCRIPTION: Thermal spring heats indoor swimming pool, outdoor soaking pool and building which includes an indoor running track and racquetball facility.

REFERENCE: Loam, 1980

SITE NO.: 207
STATE: $\quad$ MT
SITE: $\quad$ Chico Hot Springs
LOCATION: Park COUnty
APPLICATION TYPE: P $\quad 113 \mathrm{~F}$
RESOURCE TEMP.: $\quad 320 \mathrm{gpm}$
MAX. FLOW:
THERMAL CAPACITY: $\quad 0.8 \mathrm{million}$ Btu/h
ANMUAL ENERGY: $\quad 4.6$ billion Btu/y
DESCRIPTION: Thermal spring heats outdoor swiming pool, soaking pool and private baths.

REFERENCE: Loam, 1980

Site No.: 208
STATE: $\quad$ MT
SITE: $\quad$ Boulder Hot Springs
LOCATION: Boulder
APPLICATION TYPE: S, P. G
RESOURCE TEMP.: $\quad 169 \mathrm{~F}$
MAX. FLOW: $\quad 500 \mathrm{gpm}$
THERMAL CAPACITY: $\quad 1.0 \mathrm{mill}$ ion Btu/h
ANNUAL ENERGY: $\quad 4.4$ billion Btu/y
DESCRIPTION: Thermal spring is used for space heating a resort hotel facility, indoor hot baths, and an outdoor

REFERENCE: Sonderigger, 1981

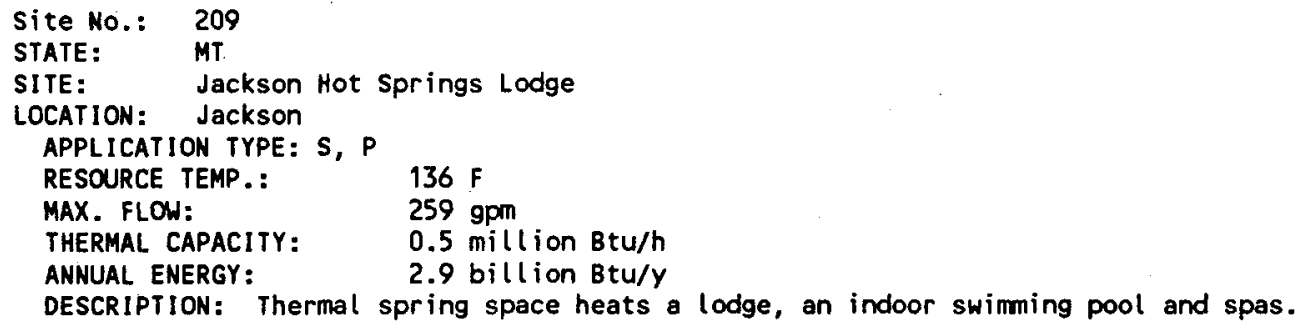

Site NO.: 210
STATE: $\quad$ MT
SITE: $\quad \begin{aligned} & \text { Medicine Hot Springs } \\ & \text { LOCATION: Conner }\end{aligned}$
APPLICATION TYPE: P $\quad 113 \mathrm{~F}$
RESOURCE TEMP.: $\quad 100 \mathrm{gpm}$
MAX. FLOW:
THERMAL CAPACITY: $\quad 0.6 \mathrm{mill}$ ion Btu/h
ANNUAL ENERGY: $\quad 2.6$ billion Btu/y
DESCRIPTION: Thermal spring heats swiming pool and three spas.


SITE: $\quad$ Sleeping Child Hot Springs

LOCATION: Ravalli County

APPLICATION TYPE: P

RESOURCE TEMP.:

MAX. FLOW:

$113 \mathrm{~F}$

THERMAL CAPACITY: $\quad 0.7 \mathrm{mill}$ ion $8 \mathrm{tu} / \mathrm{h}$

ANNUAL ENERGY: $\quad 2.5$ billion Btu/y

DESCRIPTION: Thermal spring heats an outdoor swimming pool, soaking pool and an indoor sauna.

REFERENCE : Loam, 1980

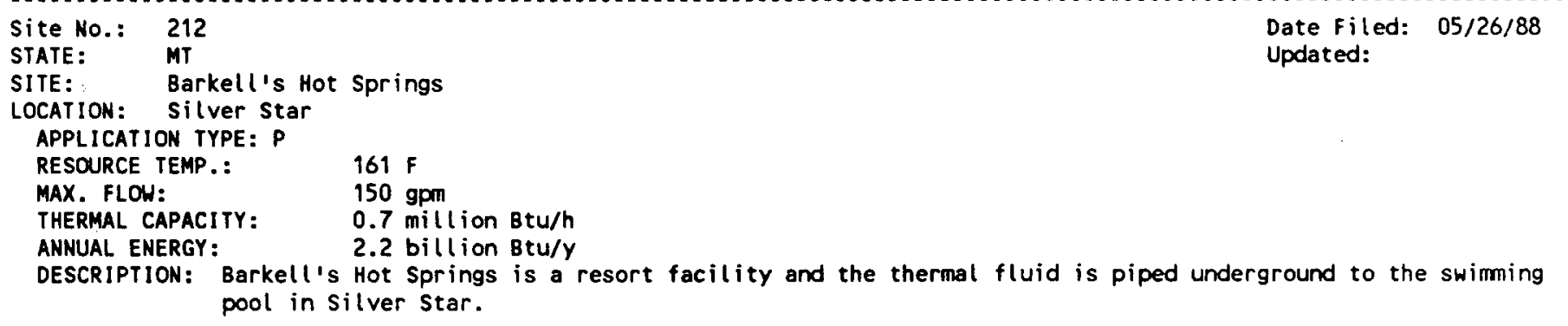

REFERENCE : Loam, 1980

$\begin{array}{lll}\text { Site No.: } & 213 & \text { Date Filed: } \\ \text { STATE: } & \text { MT } & \text { 05/26/88 } \\ \text { SITE: } & \text { New Biltmore Hot Springs } & \text { Updated: }\end{array}$

LOCATION: Madison County

APPLICATION TYPE: P

RESOURCE TEMP.: $\quad 127$ F

MAX. FLOW: $26 \mathrm{gpm}$

THERMAL CAPACITY: $\quad 0.3 \mathrm{mill}$ ion Btu/h

ANNUAL ENERGY: $\quad 1.8$ billion Btu/Y

DESCRIPIION: The thermal springs is used in a recreational spa and for a swimming pool.

REFERENCE: LOam, 1980
Site NO.: 214
STATE: $\quad$ MT
SITE: $\quad$ Ennis Hot Springs
LOCATION: Ennis
APPLICATION TYPE: $\quad 1 \quad 181 \mathrm{~F}$
RESOURCE TEMP.:
MAX. FLOW:
THERMAL CAPACITY: $\quad 0.8 \mathrm{mpm}$
ANNUAL ENERGY: $\quad 1.7 \mathrm{billion}$ Btu/h
DESCRIPTION: A shallow $(100 \mathrm{ft}$ ) geothermal well provides hot water for a laundry facility.

REFERENCE: Sonderegger, 1981

Site NO.: 215
STATE: $\quad$ MT
SITE: $\quad$ Camas Hot Springs
LOCATION: HOt Springs
APPLICATION TYPE: P
RESOURCE TEMP.: $\quad 104 \mathrm{~F}$
MAX. FLOW:
THERMAL CAPACITY: $\quad 0.2 \mathrm{gpm}$
ANNUAL ENERGY: $\quad 0.7$ billion Btu/h
DESCRIPTION: Large health center owned and operated by the Flathead Indian Tribes uses thermal spring for swimming
$\quad$ pool and bath houses.


LOCATION: ClanCY

APPLICATION TYPE: S, P

RESOURCE TEMP.: $133 \mathrm{~F}$

MAX. FLOW: $\quad 100 \mathrm{gpm}$

THERMAL CAPACITY: $\quad 0.2 \mathrm{mill}$ ion Btu/h

ANNUAL ENERGY: $\quad 0.6$ billion $8 t u / Y$

DESCRIPTION: Thermal spring provides space heating for nursing home and an indoor therapeutic pool.

REFERENCE: Sonderegger, 1981

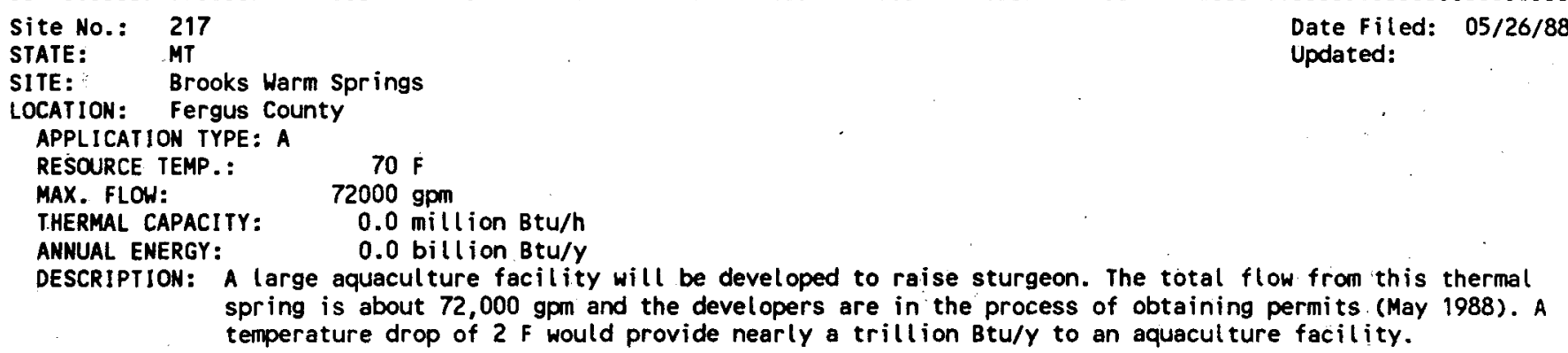

REFERENCE: Culver, 1988

Site No.: 218
STATE:
SITE:
LOCATION: Northern Part of State
APPLICATION TYPE: HP
RESOURCE TEMP.: $\quad 52 \mathrm{~F}$
MAX. FLOW: $\quad 2400 \mathrm{gpm}$
THERMAL CAPACITY: $\quad 23.4 \mathrm{mill}$ ion Btu/h
ANNUAL ENERGY: $\quad 41.0$ billion Btu/y
DESCRIPTION: An est imated 1000 heat pump wells are used for space heating and cooling. The reported annual energy is
for heat load only, includes 30 percent ground water and 35 percent vertical earth coupled heat pump
wells. The remainder are horizontal earth coupled and are not included.

REFERENCE: Eversoll, 1988 \& Ellis, 1988

Site NO:: 219
STATE: $\quad$ NB
SITE: $\quad$ Homestead National Monument
LOCATION: Beatice
APPLICATION TYPE: HP
RESOURCE TEMP.:
MAX. FLOW:
THERMAL CAPACITY: $\quad 58 \mathrm{~F}$
ANNUAL ENERGY: $\quad 0.2 \mathrm{mill}$ Upm Btu/h
DESCRIPTION: A heat pump well is used for space heating and cooling of the administrative building.

REFERENCE: MCMUTray, 1988

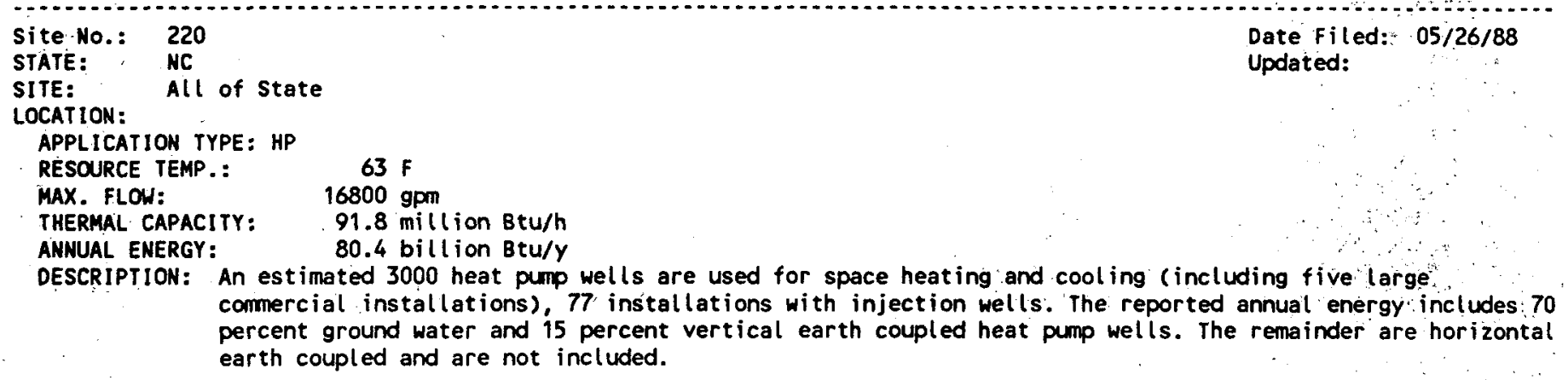


SITE: Secondary Oil Recover

LOCATION: southeastern area of ND

APPLICATION TYPE: I

RESOURCE TEMP.: $170 \mathrm{~F}$

MAX. FLOW: $1500 \mathrm{gpm}$

THERMAL CAPACITY: $\quad 75.1 \mathrm{mill}$ ion $8 \mathrm{tu} / \mathrm{h}$

ANNUAL ENERGY: $\quad 526.2$ billion Btu/y

OESCRIPTION: Geothermal fluids are injected into oil wells to move oil towards production wells. This heat is extremely useful in increasing the recovery of up to an additional third of the original oil.

REFERENCE: Reed, 1988

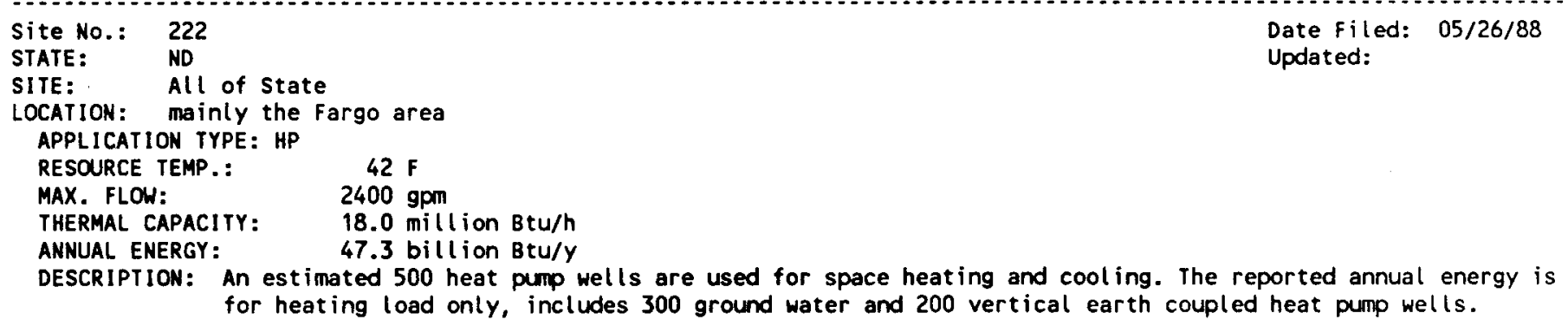

REFERENCE: NWWA, 1988; Ell is, 1988

Site NO.: 223
STATE:
SITE:
LOCATION: Farton School
APPLICATION TYPE: HP
RESOURCE TEMP.:
MAX. FLOW:
THERMAL CAPACITY:
ANNUAL ENERGY: $1.2 \mathrm{million}$ Btu/h
DESCRIPTION: A 100 ton vertical closed loop earth coupled (100 bore holes) heat pump is used for space heating and
Cooling. The reported annual energy is only for the heating load.

REFERENCE: Ellis, 1988

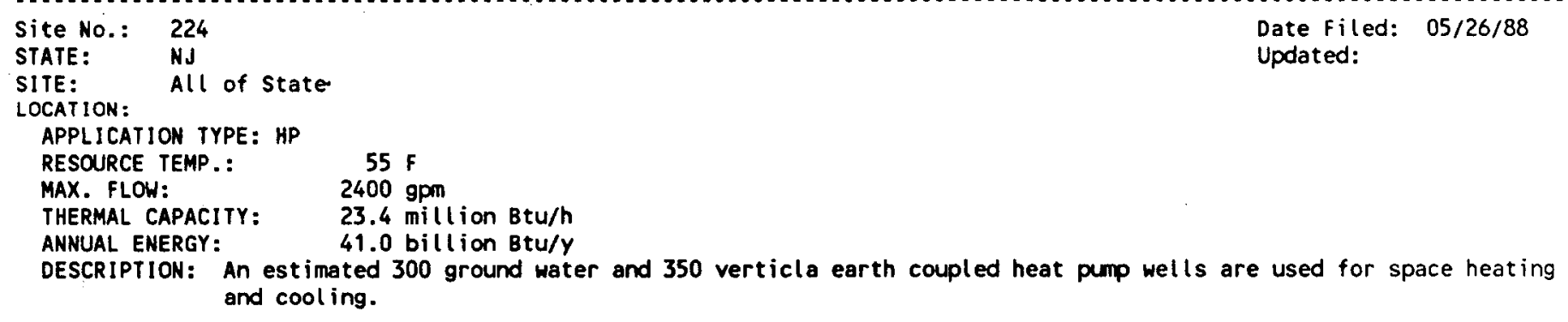

REFERENCE: Ellis, 1988

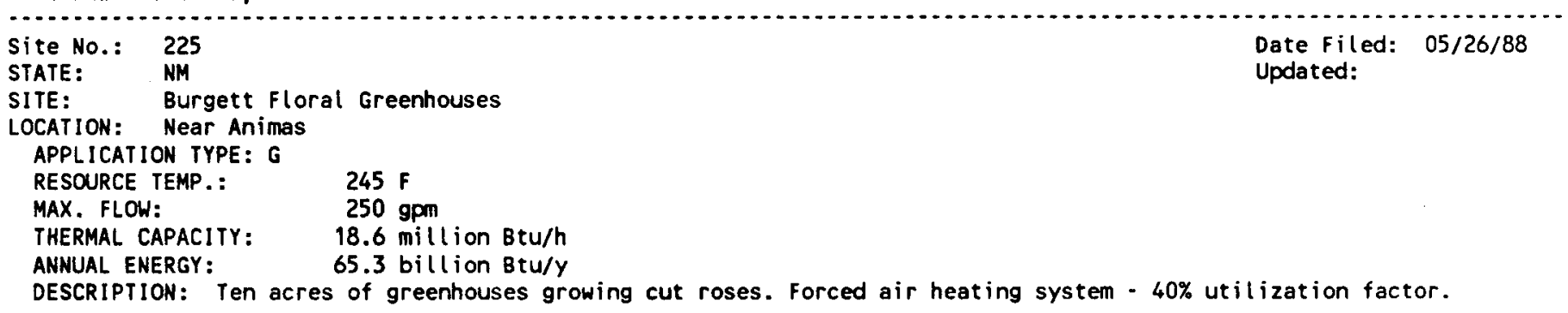


APPLICATION TYPE: S, DHW

RESOURCE TEMP.: $142 \mathrm{~F}$

MAX. FLOW: $\quad 417 \mathrm{gpm}$

THERMAL CAPACITY: $\quad 3.7 \mathrm{mill}$ ion $\mathrm{Btu} / \mathrm{h}$

ANNUAL ENERGY: $\quad 45.9$ billion Btu/Y

DESCRIPTION: Domestic hot water up to $400 \mathrm{gpm}$ swimming pool heating, space heating and a small greenhouse are heated by wells $850-950 \mathrm{ft}$ deep.

REFERENCE: CUnniff NMSU DEmo Proj DOE/IP

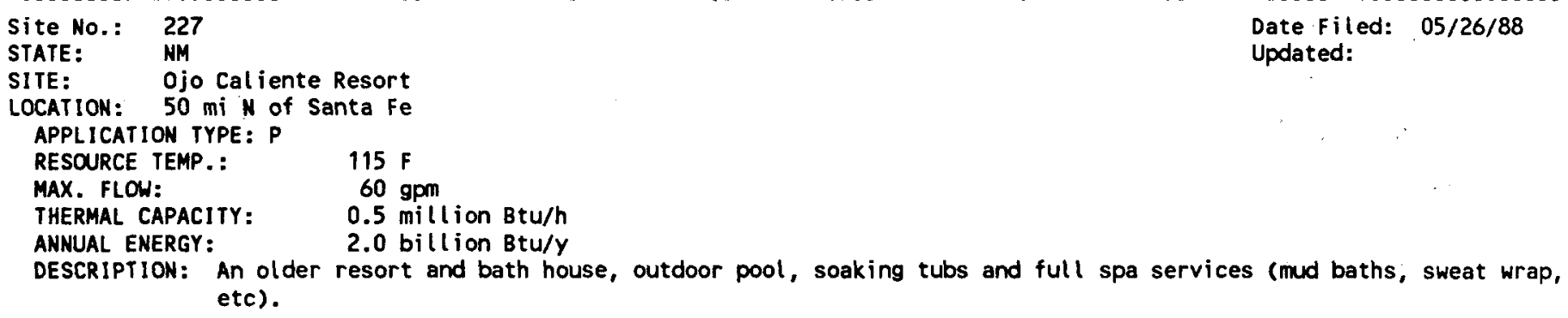

REFERENCE: LOam, 1985

Site NO.: 228
STATE:
SITE: NM
LOCATION: Jemez Springs

REFERENCE: Lienau, 1986; Loam, 1985

Site No.: 229
STATE: $\quad$ NM
SITE: $\quad$ Truth or Consequences
LOCATION:
APPLICATION TYPE: P
RESOURCE TEMP.: $\quad 113 \mathrm{~F}$
MAX. FLOW:
THERMAL CAPACITY: $\quad$ 9Pm
ANNUAL ENERGY:
DESCRIPTION: At least 8 natural mineral water bath houss are located in the hot springs area. Most are older and
small, having only $3-9$ spa/soaking tubs therapy and massage.

REFERENCE : Loam, 1985

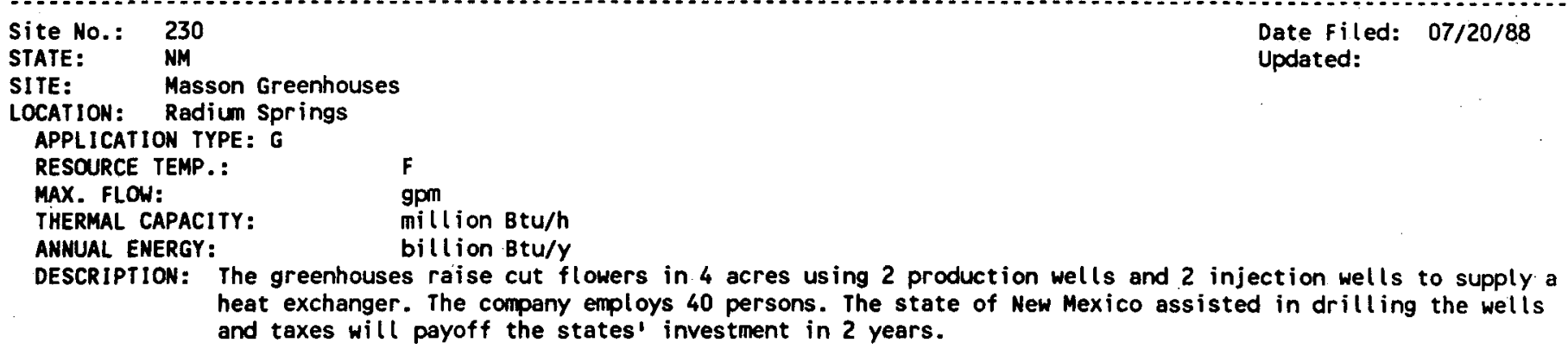


SITE: $\quad$ Round Mountain Gold Corp

LOCATION: Smoky Valley, NV APPLICATION TYPE: I RESOURCE TEMP.: MAX. FLOW:

DESCRIPTION: Use $186 \mathrm{~F}$ geothermal fluide to heat cyanide circuit $(2000 \mathrm{pgm})$ from $42 \mathrm{~F}$ to $72 \mathrm{~F}$. Injection of spent geothermal fluids.

REFERENCE: Loam.1985; Trexler, 1988

Site No.: 233
STATE: $\quad$ NV
SITE: $\quad$ Geothermal Food Processors
LOCATION: Brady Hot Springs E. of Fernly
APPLICATION TYPE: I $\quad 270 \mathrm{~F}$
RESOURCE TEMP.: $\quad 1000 \mathrm{gPm}$
MAX. FLOW:
THERMAL CAPACITY: $\quad 19.0 \mathrm{mill}$ ion Btu/h
ANNUAL ENERGY: $\quad 86.0$ billion Btu/y
DESCRIPTION: A food dehydration facility drying onions, garlic, celery, carrots. In operation since 1978.

REFERENCE: Lienau. 1986

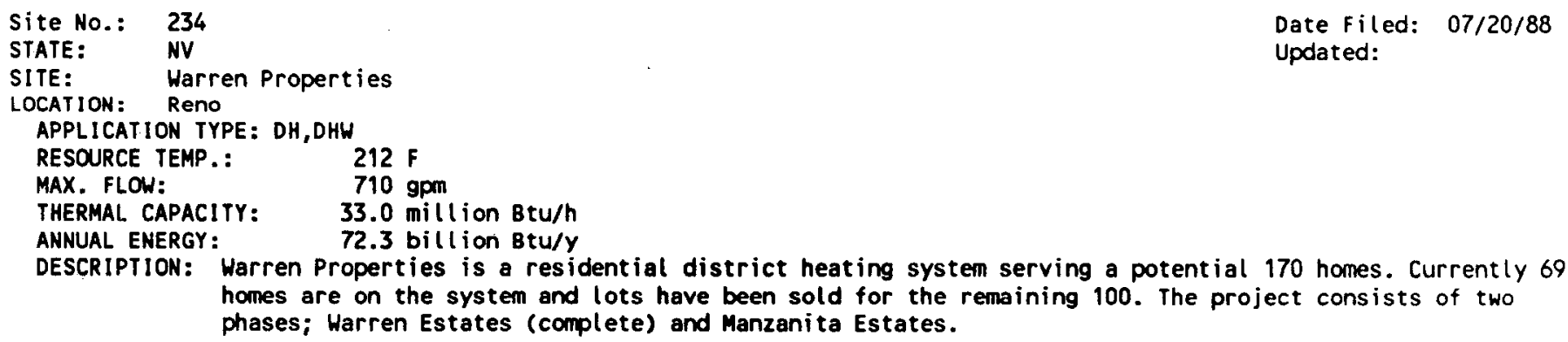


SITE: $\quad$ Warm Spring Resort

LOCATION: $50 \mathrm{mi} . \mathrm{N}$. Of Las Vegas

APPLICATION TYPE: $P$

RESOURCE TEMP.: $90 \mathrm{~F}$

MAX. FLOW:

THERMAL CAPACITY: $\quad 8.0 \mathrm{mill}$ ion Btu/h

ANNUAL ENERGY: $\quad 63.0 \mathrm{billion} B t u / y$

DESCRIPTION: Owned by a nationwide campers organization - RV hook-ups and camping only for members: Grounds and pools open to public for day use. Several natural swim pools, a large man-made swim pool and hydro pool.

REFERENCE: Loam, 1980; Trexler, 1988

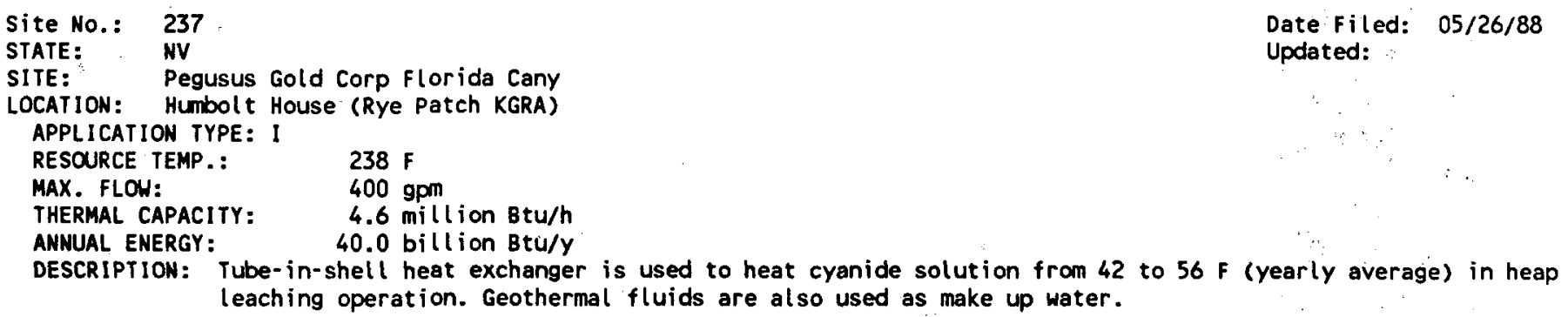

REFERENCE: Trexler, 1988; Beck 1988

SIte NO.: 238
STATE:
SITE:
LOCATION: Elko Disrict Heat
APPLICATION TYPE: DH
RESOURCE TEMP.:
MAX. FLON:
THERMAL CAPACITY:
ANNUAL ENERGY: $178 \mathrm{~F}$
DESCRIPTION: The system serves eight businesses and buildings and also the sewage digester plant.

REFERENCE: Lienau, 1986

$\begin{array}{ll}\text { Site No.: } & 239 \\ \text { STATE: } & \text { NV } \\ \text { SITE: } & \text { Reno-Moana Area }(200) \\ \text { LOCATION: Reno } & \\ \text { APPLICATION TYPE: S } & 120 \mathrm{~F} \\ \text { RESOURCE TEMP.: } & 0 \mathrm{gpm} \\ \text { MAX. FLOW: } & 14.0 \mathrm{million} \mathrm{Btu/h} \\ \text { THERMAL CAPACITY: } & 31.8 \mathrm{billion} \text { Btu/y } \\ \text { ANNUAL ENERGY: } & \end{array}$

DESCRIPTION: Approximately 200 homes in the Moana area are heated using down-hole heat exchangers. Some of the wells pump small amounts to maintain temperature in the wells. The use is expanding rather fast - at times one home every two weeks.

REFERENCE : MCKay Drillers; MCKay Geotherm

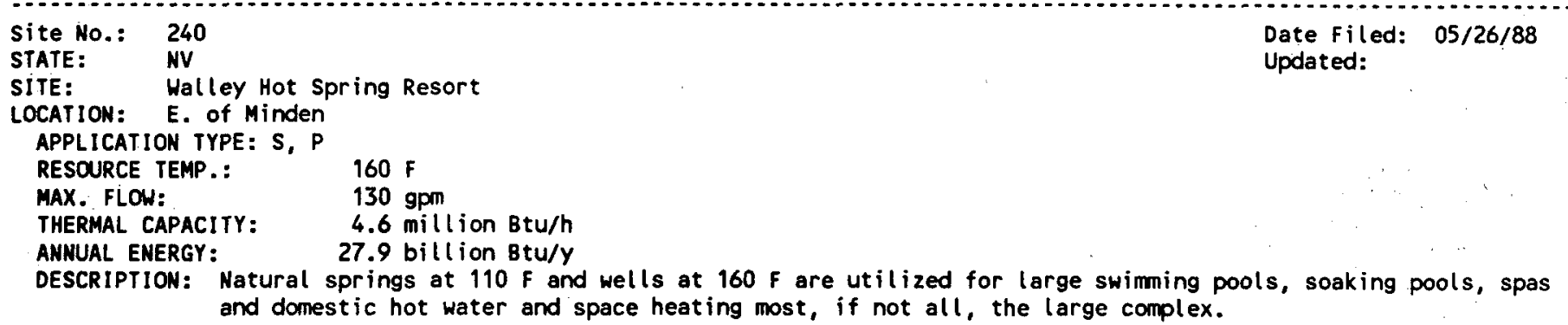


SITE: $\quad$ Elko County School District

LOCATION: EIKO

APPLICATION TYPE: DH,S, KP

RESOURCE TEMP.: $\quad 190 \mathrm{~F}$

MAX. FLOW: $\quad 300 \mathrm{gpm}$

THERMAL CAPACITY: $\quad 7.2 \mathrm{mill}$ (lion Btu/h

ANNUAL ENERGY: $15.7 \mathrm{billi}$ : $\mathrm{Btu} / \mathrm{Y}$

DESCRIPTION: The ECSD district heating system started operating in 1986. A loop from a central plate heat exchanger delivers $180 \mathrm{~F}$ water to the school administration offices, gym., convention center, hospital, municipal pool, city hall and Jr. High School (heat pump system). An impressive $100 \mathrm{~F}$ temperature drop is achieved across the system and disposal is to percolation ponds and golf course irrigation.

REFERENCE: Rafferty, 1988

Site No.: 242

STATE: $\quad$ NV

SITE: $\quad$ Hobo Hot Springs

LOCATION: $9 \mathrm{mi}$. SO. Of Carson City

APPLICATION TYPE: A

RESOURCE TEMP.: $\quad 106 \mathrm{~F}$

MAX. FLOW: $\quad 100 \mathrm{gPm}$

THERMAL CAPACITY: $\quad 2.0$ million Btu/h

ANNUAL ENERGY: $\quad 14.4$ billion $8 \mathrm{tu} / \mathrm{y}$

DESCRIPTION: Tropical fish production. See article in GRC Trans. V.11, pg 3.
Date filed: $05 / 26 / 88$ Updated:

REFERENCE: Trexler, 1988

Site NO.: 243
STATE:
SITE: NV
LOCATION: Renren Estates
APPLICATION TYPE: DH
RESOURCE TEMP.: $\quad 209 \mathrm{~F}$
MAX. FLOW: $\quad 1000 \mathrm{gpm}$
THERMAL CAPACITY: $\quad 3.6 \mathrm{million}$ Btu/h
ANNUAL ENERGY: $\quad 7.9$ billion Btu/Y
DESCRIPTION: This district heating system supplis space heating and DHW for residences in the development. Forty
homes are currently on line. Ninety more lots are available. The system is always expanding as new homes
are built.
are built.

REFERENCE: NOCS, 1987

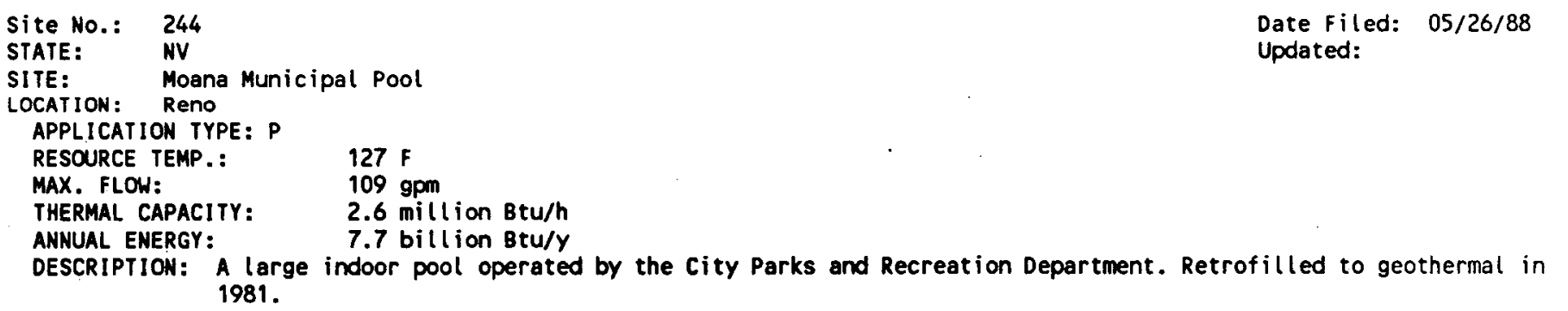

REFERENCE: Rafferty, 1982

Site No.: 245
STATE: $\quad$ NV
SITE: $\quad$ Caliente City Pool


APPLICATION TYPE: P

RESOURCE TEMP.: $\quad 120 \mathrm{~F}$

MAX. FLOW: 75 gpm

THERMAL CAPACITY: $\quad 0.8 \mathrm{million}$ Btu/h

ANMUAL ENERGY: $\quad 5.7$ billion Btu/Y

DESCRIPTION: This old resort has an outdoor pool and nine large soaking pools.

REFERENCE: Loam, 1980; Trexler, 1988

Site No.: $\quad 247$
STATE: $\quad$ NV
SITE: Bowers Mansion
LOCATION: CarSOn City
APPLICATION TYPE: P $\quad 116 \mathrm{~F}$
RESOURCE TEMP.: $\quad 75 \mathrm{gpm}$
MAX. FLOW: $\quad \begin{aligned} & \text { Date Filed: } \\ & \text { Updated: }\end{aligned}$
THERMAL CAPACITY: $\quad 1.4 \mathrm{million}$ Btu/h
ANMUAL ENERGY: $\quad 4.7$ billion Btu/y
DESCRIPTION: A Washoe County park with large geothermal pool. Open only part of the year.

DESCRIPIION: A Washoe County park with large geothermal pool. Open only part of the year.

REFERENCE: Loam, 1980; Trexter, 1988

Site No.: 248
STATE: $\quad$ NV
SITE: $\quad$ Brockway Springs Resort
LOCATION: N. Shore of Lake Tahoe
APPLICATION TYPE: P $\quad 180 \mathrm{~F}$
RESOURCE TEMP.: $\quad 150 \mathrm{gpm}$
MAX. FLOW:
THERMAL CAPACITY: $\quad 0.5 \mathrm{mill}$ Updated:
ANNUAL ENERGY: $\quad 3.9$ billion Btu/h
DESCRIPTION: Once a large resort hotel, it has been restored with additions, and now is a condominium. Six springs
$\quad$ feed a large coffer dam pool in Lake Tahoe.

REFERENCE : Kaysing, 1984

$\begin{array}{ll}\text { Site No.: } & 249 \\ \text { STATE: } & \text { NV } \\ \text { SITE: } & \text { Caliente Hot Springs Motel } \\ \text { LOCATION: Caliente } & \\ \text { APPLICATION TYPE: P } & \\ \text { RESOURCE TEMP.: } & 115 \mathrm{~F} \\ \text { MAX. FLOW: } & 40 \mathrm{gpm} \\ \text { THERMAL CAPACITY: } & 0.4 \mathrm{milli} \text { B Btu/h } \\ \text { ANHUAL ENERGY: } & 2.4 \text { billion Btu/y }\end{array}$

Date Filed: $05 / 26 / 88$ Updated:

REFERENCE: Loam, 1980; Trexler, 1988

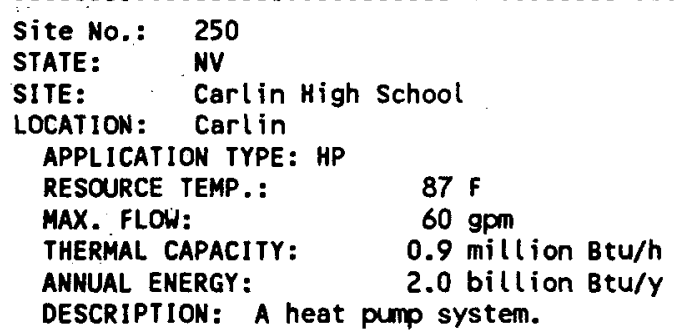


LOCATION: Hells

APPLICATION TYPE: HP

RESOURCE TEMP.:

MAX. FLOW:

$87 \mathrm{~F}$

THERMAL CAPACITY:

50 gpm

ANNUAL ENERGY:

$0.8 \mathrm{mill}$ ion Btu/h

1.6 billion 8 tu/y

DESCRIPTION: A ground water heat pump system is used for space heating and cooling.

REFERENCE : Lienau, 1986

$\begin{array}{ll}\text { Site No.: } & 252 \\ \text { STATE: } & \text { NV } \\ \text { SITE: } & \text { Medical Center } \\ \text { LOCATION: Caliente } & \\ \text { APPLICATION TYPE: } & \\ \text { RESOURCE TEMP.: } & 167 \mathrm{~F} \\ \text { MAX. FLOW: } & 46 \mathrm{gPm} \\ \text { THERMAL CAPACITY: } & 0.7 \mathrm{million} \mathrm{Btu} / \mathrm{h} \\ \text { ANNUAL ENERGY: } & 1.5 \mathrm{bill} \text { ion } \mathrm{Btu} / \mathrm{y}\end{array}$

DESCRIPTION: This medical center/small hospital was electrically heated and retrofilled to utilize geothermal.

REFERENCE: Lienau, 1986

Site NO.: 253
STATE:
SITE:
LOCATION: Wabuska
APPLICATION Of Yerrington
RESOURCE TEMP.: $\quad 270 \mathrm{~F}$
MAX. FLOW:
THERMAL CAPACITY: $\quad 0.0 \mathrm{mill}$
ANNUAL ENERGY:
DESCRIPTION: A small aquaculture facility utilizes some effluent from a binary power plant. Various species, mostly
$\quad$ tropicals, are grown.

REFERENCE : Culver, 1988

$\begin{array}{ll}\text { Site No.: } & 254 \\ \text { STATE: } & \text { NV } \\ \text { SITE: } & \text { Lakeview Apartments } \\ \text { LOCATION: Reno } & \\ \text { APPLICATION TYPE: DHW } \\ \text { RESOURCE TEMP.: } \\ \text { MAX. FLOW: } & \\ \text { THERMAL CAPACITY: } & \text { gPm } \\ \text { ANNUAL ENERGY: } & \text { million Btu/h } \\ \text { DESCRIPIION: A number of apartment build }\end{array}$

DESCRIPIION: A number of apartment buildings will be retrofitted to geothermal domestic hot water (DHW).

REFERENCE: MacKoy, 1988

Site NO.: 255
STATE: $\quad$ NV
SITE: $\quad$ Baileys Hot Springs
LOCATION: E. of Death Valley Nat'l Monu.
APPLICATION TYPE: P
RESOURCE TEMP.: $\quad 160 \mathrm{~F}$
MAX. FLOW:
THERMAL CAPACITY: $\quad$ gpm
ANNUAL ENERGY: $\quad$ million Btu/h
DESCRIPTION: Primarily a restaurant, bar, casino and RV park. Has three large pools, formerly railraod water storage
$\quad$ reservoirs; now used as pools.


SITE: Wells Rural Electric

LOCATION: Hells

APPLICATION TYPE: HP

RESOURCE TEMP.: F

MAX. FLOW: gpm

THERMAL CAPACITY: million Btu/h

ANNUAL ENERGY: billion Btu/Y

DESCRIPTION:

REFERENCE: Petty, 1988

Site No.: 257
STATE:
SITE:
LOCATION: CQUa Caliente Trailer Park

REFERENCE : Culver, 1988

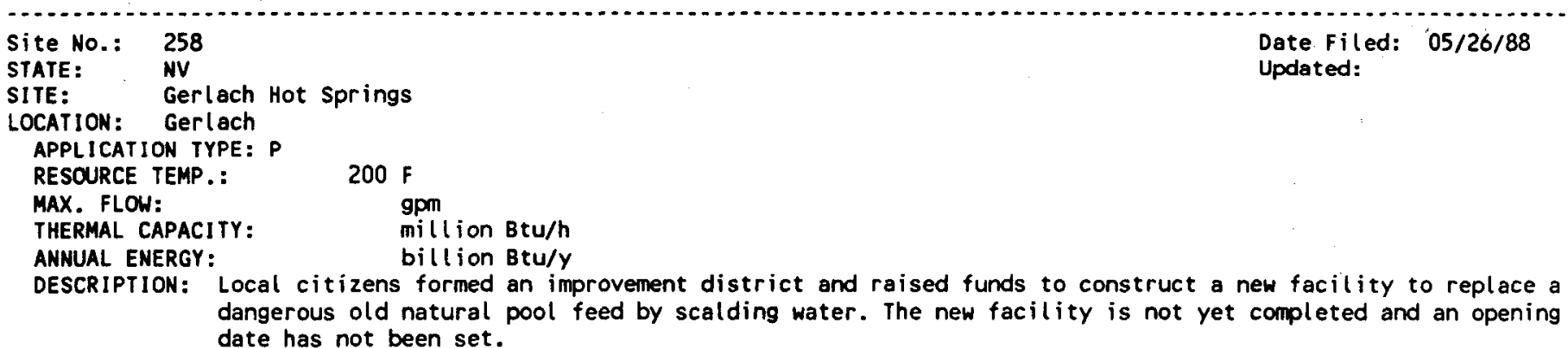

REFERENCE: Loam 1980; Minto, 1988

$\begin{array}{ll}\text { Site NO.: } & 259 \\ \text { STATE: } & \text { NV } \\ \text { SITE: } & \text { Elko Jr. High School } \\ \text { LOCATION: Elko } & \\ \text { APPLICATION TYPE: } & \\ \text { RESOURCE TEMP.: } & 190 \mathrm{~F} \\ \text { MAX. FLOW: } & 300 \mathrm{gpm} \\ \text { THERMAL CAPACITY: } & \text { million Btu/h } \\ \text { ANNUAL ENERGY: } & \text { billion Btu/Y }\end{array}$

DESCRIPTION:

REFERENCE: Petty, 1988

Site NO:: 260
STATE: $\quad$ NV
SITE: Salem Plaza
LOCATION: RenO
APPLICATION TYPE: S,DHW
RESOURCE TEMP.: $\quad 160 \mathrm{~F}$
MAX. FLOH:
THERMAL CAPACITY: $\quad$ million Btu/h
ANNUAL ENERGY: $\quad$ billion Btu/y
DESCRIPTION: Salem Plaza will be retrofitted to geothermal space heating using a production well $(1,500 \mathrm{ft})$ and an


SITE: Steamboat Springs (Spa)

LOCATION: S. Of RENO

APPLICATION TYPE: S, P

RESOURCE TEMP.: $\quad 200$

MAX. FLOW: $\quad 590 \mathrm{gpm}$

THERMAL CAPACITY: million Btu/h

ANWUAL ENERGY: billion BtU/Y

DESCRIPTION: Only a very small portion of the flow is used for spas (supervised by registered nurse) and space heating.

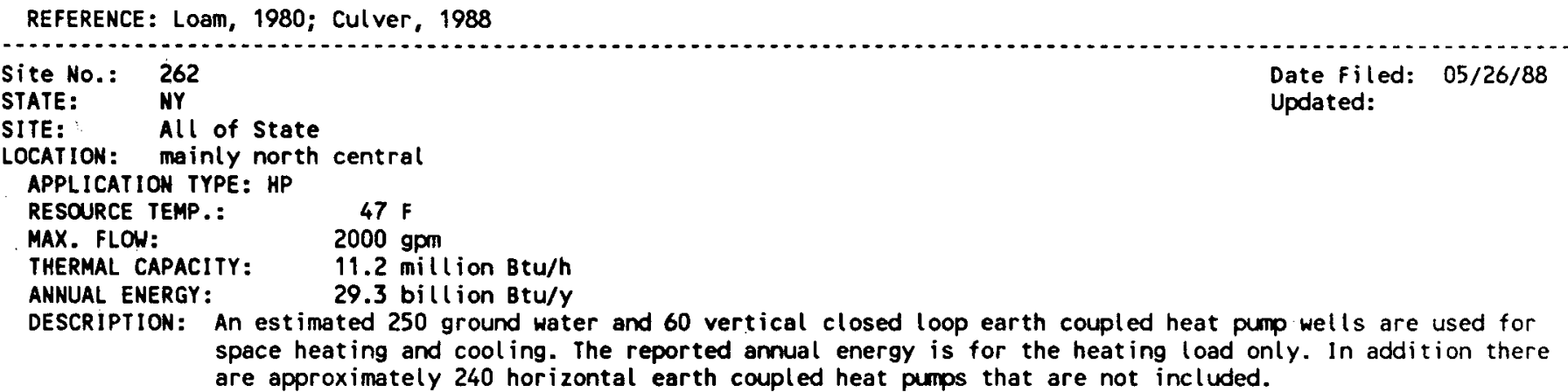

REFERENCE:' Krachow, 1988 \& Ellis, 1988

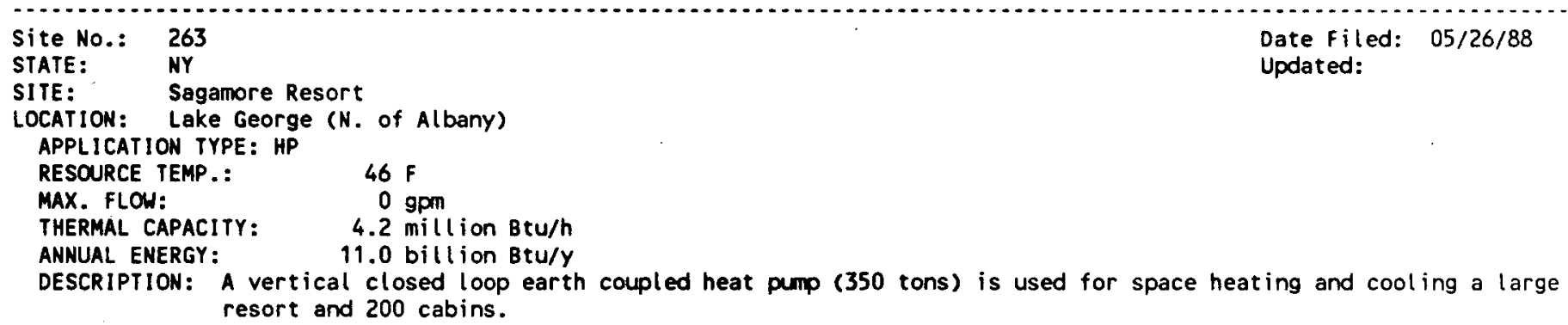

REFERENCE: Ellis, 1988

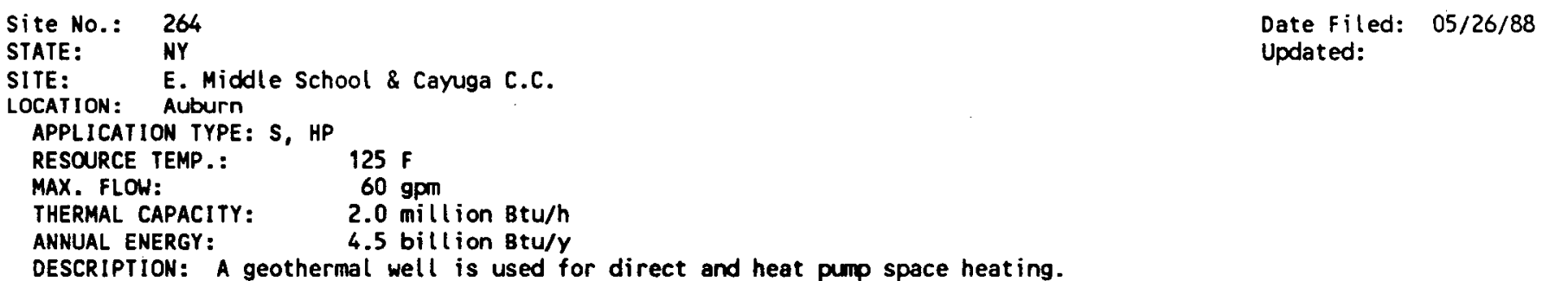

REFERENCE: Krachow, 1988

Site No.: 265
STATE: $\quad$ OH
SITE: $\quad$ All of State
LOCATION:
APPLICATION TYPE: HP
RESOURCE TEMP.: $\quad 53 \mathrm{~F}$
MAX. FLOW: $\quad 25600 \mathrm{gpm}$
THERMAL CAPACITY: $\quad 129.6 \mathrm{mill}$ ion Btu/h
ANMUAL ENERGY: $\quad 227.0 \mathrm{bill}$ ion Btu/Y
DESCRIPTION: An estimated 3200 ground water and 400 vertical closed loop earth coupled heat pump wells are used for
space heating and cooling. The reported annual energy is for the heat ing load only.


SITE: Central Part of State

LOCATION:

APPLICATION TYPE: HP

RESOURCE TEMP.:

MAX. FLOW:

THERMAL CAPACITY:

ANNUAL ENERGY:

240 gpm

$1.2 \mathrm{mill}$ ion Btu/h

1.0 billion Btu/y

DESCRIPTION: An estimated 30 heat pump wells:are used for residential space heating and cooling.

REFERENCE: LUze, 1988

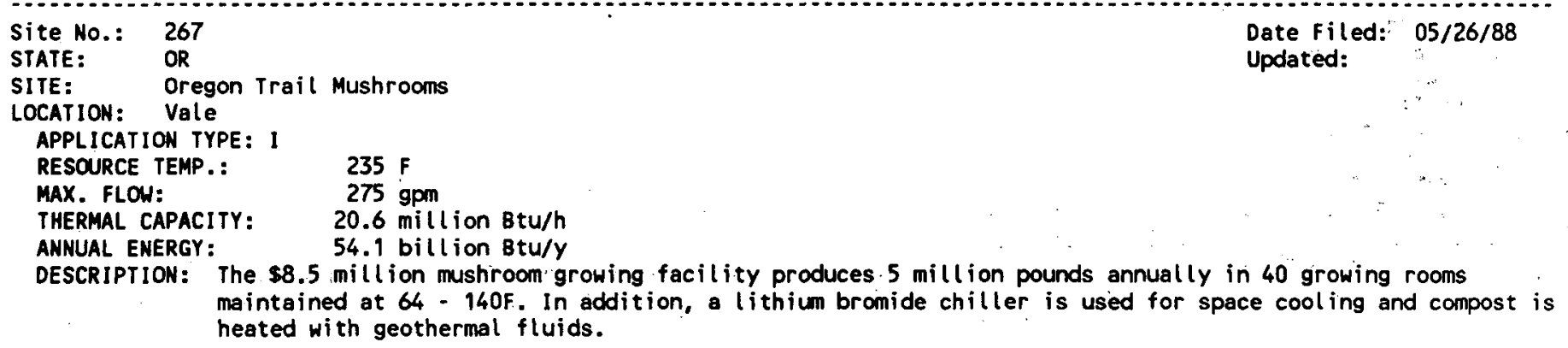

REFERENCE: Rutten, 1987

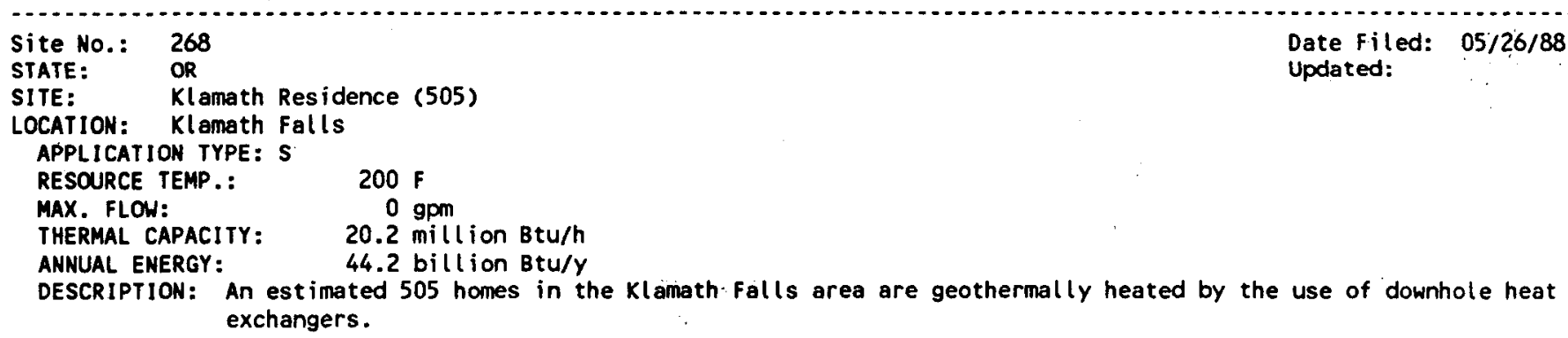

REFERENCE: LienUa, 1986

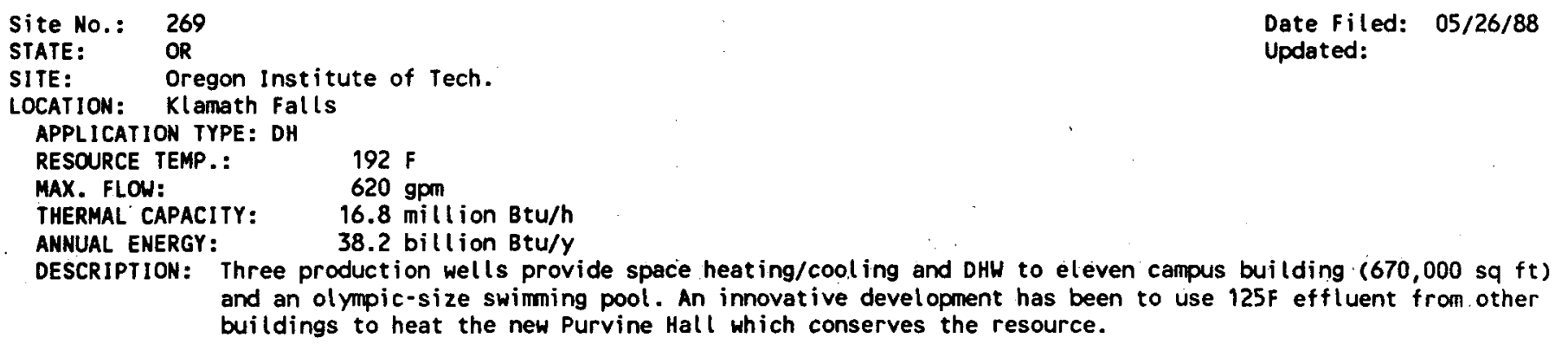

REFERENCE : Lienau, 1986

Site No.: 270
STATE: $\quad$ OR
SITE: $\quad$ Kah-nee-ta
LOCATION: Marm Springs
APPLICATION TYPE: P, OHW
RESOURCE TEMP.: $\quad 126 \mathrm{~F}$
MAX: FLOW: $\quad 450 \mathrm{gpm}$
THERMAL CAPACITY: $\quad 4.5 \mathrm{million}$ Btu/h
ANNUAL ENERGY: $\quad 27.6 \mathrm{bill}$ ion Btu/y
DESCRIPTION: Thermal spring is used to heat the swimming pool, mineral baths, DHW, and tribal bathhouse.


SITE: Merle West Medical Center

LOCATION: Klamath Falls

APPLICATION TYPE: S

RESOURCE TEMP.: $\quad 191 \mathrm{~F}$

MAX. FLOW: $\quad 325 \mathrm{gpm}$

THERMAL CAPACITY: $\quad 10.5 \mathrm{mill} \quad \mathrm{lion} B \mathrm{Btu} / \mathrm{h}$

ANNUAL ENERGY: $23.9 \mathrm{bill}$ ion $\mathrm{Btu} / \mathrm{Y}$

DESCRIPTION: One production well provides space and DHW to the medical center and the Klamath County Nursing Home.

REFERENCE: Lienau, 1986

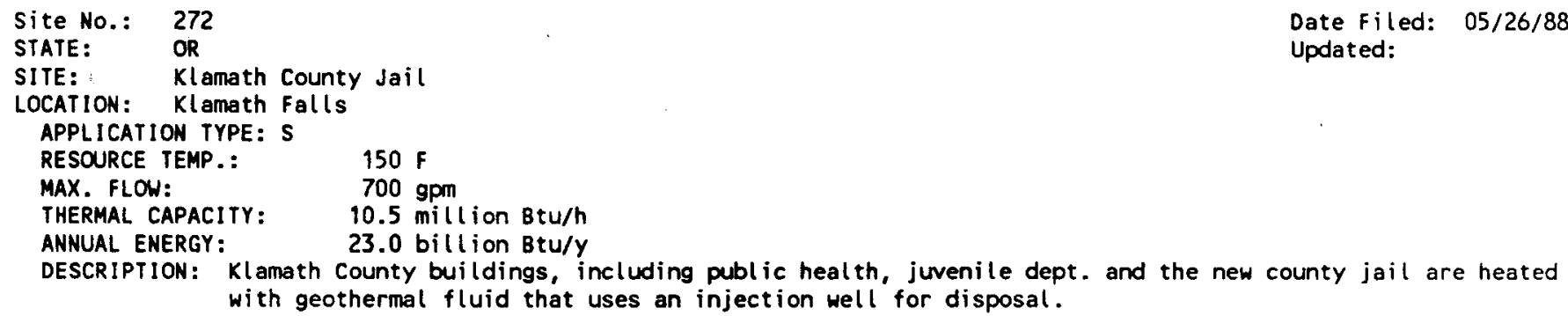


LOCATION: Klamath County

APPLICATION TYPE: S

RESOURCE TEMP.:

MAX. FLOW:

$118 \mathrm{~F}$

$450 \mathrm{gpm}$

THERMAL CAPACITY: $\quad 3.0 \mathrm{mill}$ ion Btu/h

ANNUAL ENERGY: $\quad 6.8$ billion Btu/Y

DESCRIPTION: A plant that manufactures louvered doors uses geothermal for space heating.

REFERENCE: Lienau, 1986

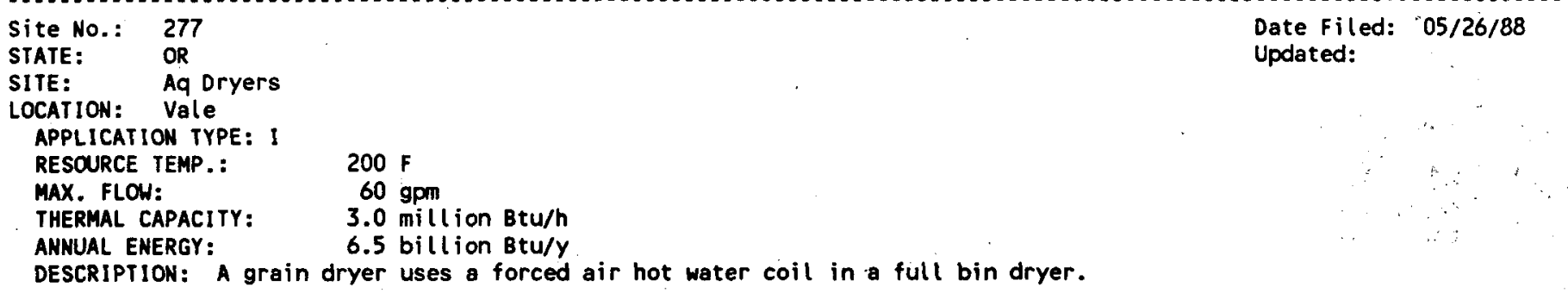

REFERENCE: Gannett, 1988

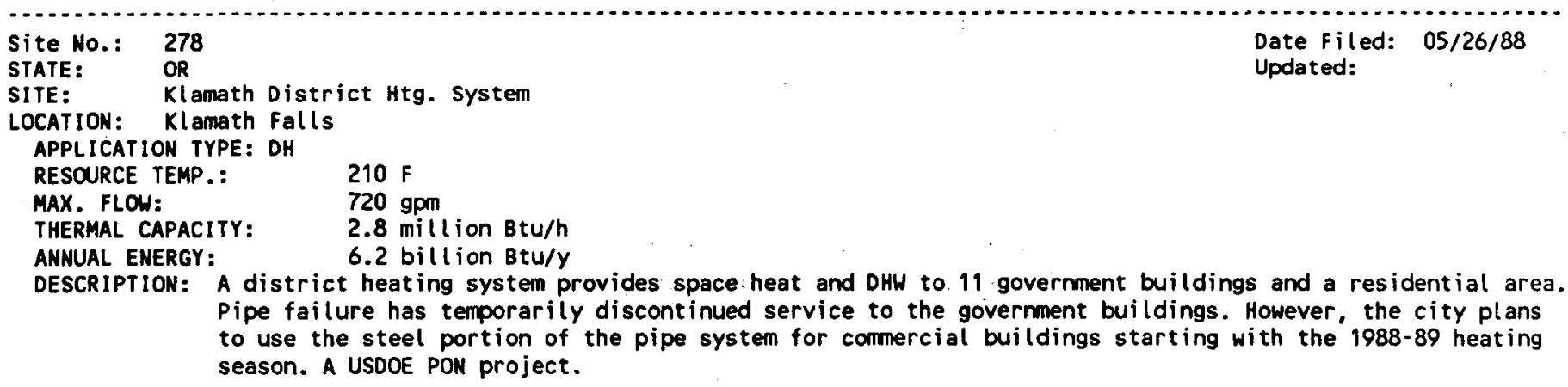

REFERENCE: Lienau, 1986

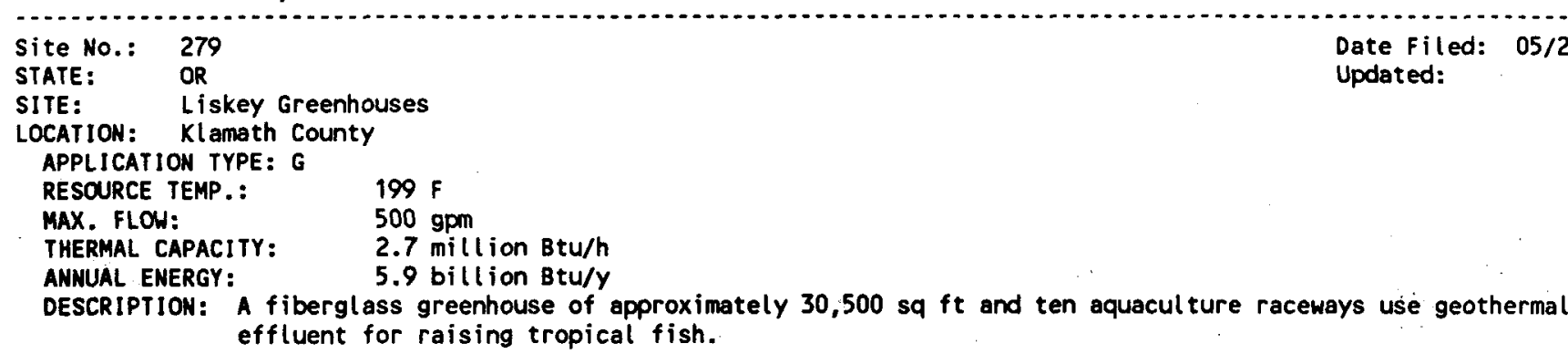

REFERENCE: Lienau, 1986

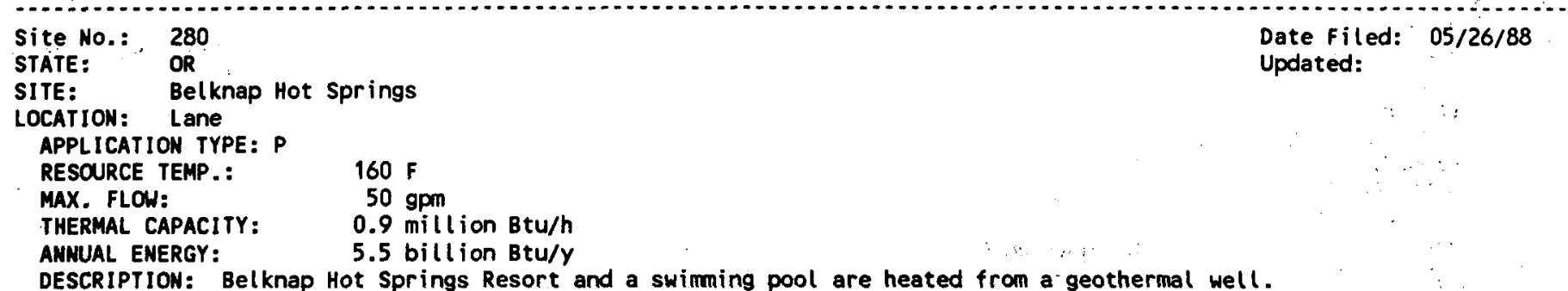

DESCRIPTION: Belknap Hot Springs Resort and a swimming pool are heated from a geothermal well. 
SITE: Jackson Hot Springs

LOCATION: AShland

APPLICATION TYPE: S, P

RESOURCE TEMP.: $\quad 111 \mathrm{~F}$

MAX. FLOW: $\quad 264 \mathrm{gpm}$

THERMAL CAPACITY: $\quad 0.7 \mathrm{mill}$ ion Btu/h

ANNUAL ENERGY: $\quad 4.4$ bill ion Btu/Y

DESCRIPTION: Thermal spring is used for space heating a motel and swimming pool.

REFERENCE : NOAA, 1982

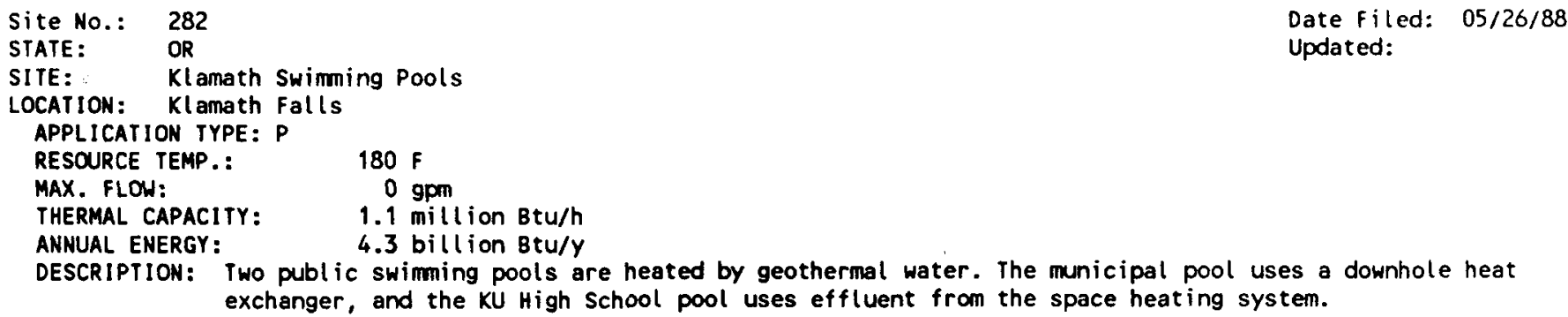

REFERENCE: Lienau, 1986

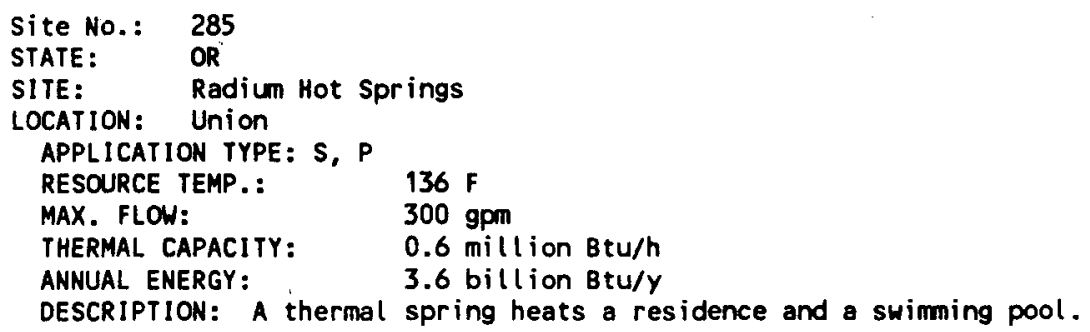


SITE: Klamath CO. Shops

LOCATION: Klamath County

APPLICATION TYPE: S

RESOURCE TEMP.: $\quad 118$ F

MAX. FLOW: $\quad 113 \mathrm{gpm}$

THERMAL CAPACITY: $\quad 1.6 \mathrm{mill}$ ion $\mathrm{Btu} / \mathrm{h}$

ANHUAL ENERGY: , $3.6 \mathrm{bill}$ ion $\mathrm{Btu} / \mathrm{Y}$

DESCRIPTION: Klamath County shops use a radiant floor system for space heating.

REFERENCE: Lienau, 1986

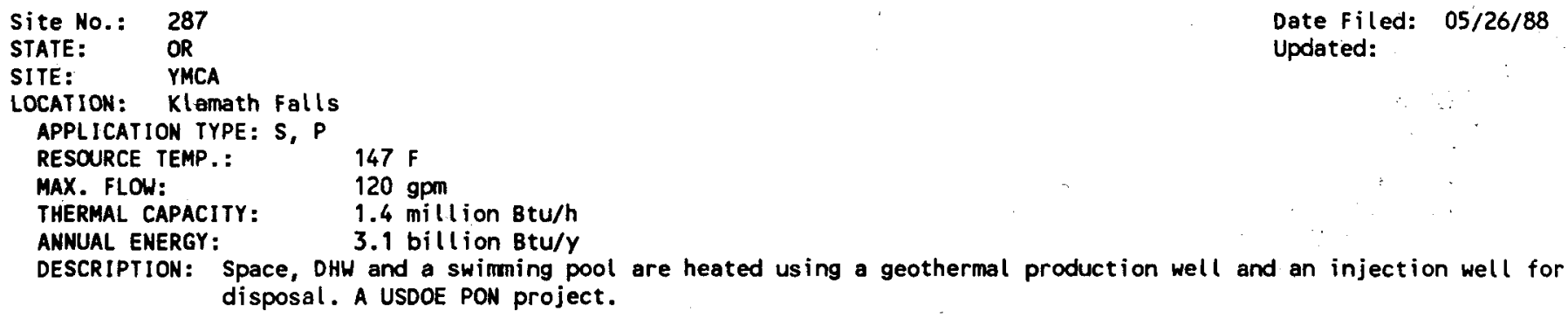

REFERENCE : Lienau, 1986

Site NO.: 288
STATE:
OR

REFERENCE : Lund, 1976

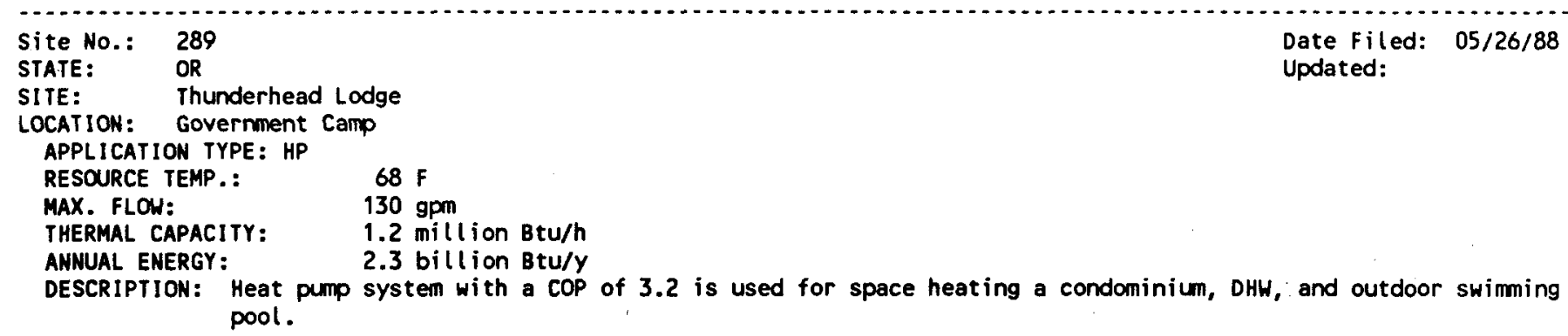

REFERENCE: Lienau, 1986

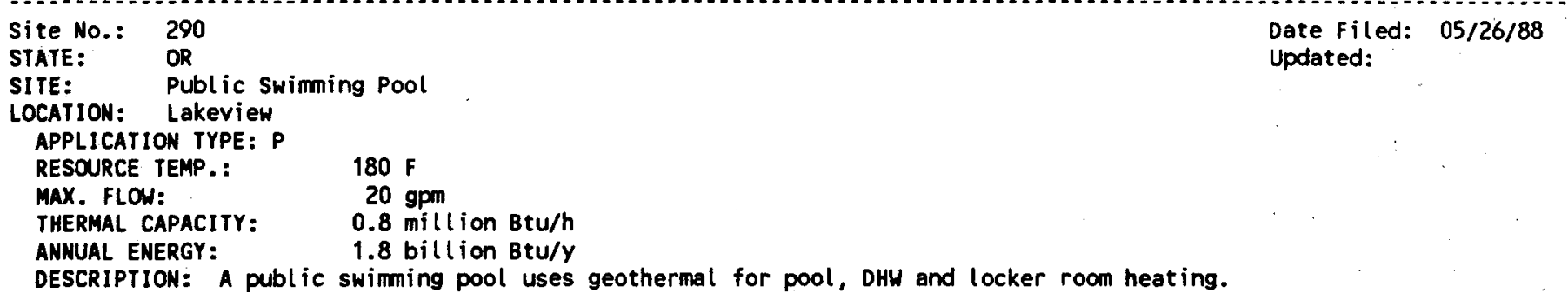


DESCRIPTION: A thermal spring provides space heating to the Hot Lake Hotel and $1320 \mathrm{ft}$. transmission line delivers heat to space heating, DHW, hot tubs and a swimning pool at the RV part.

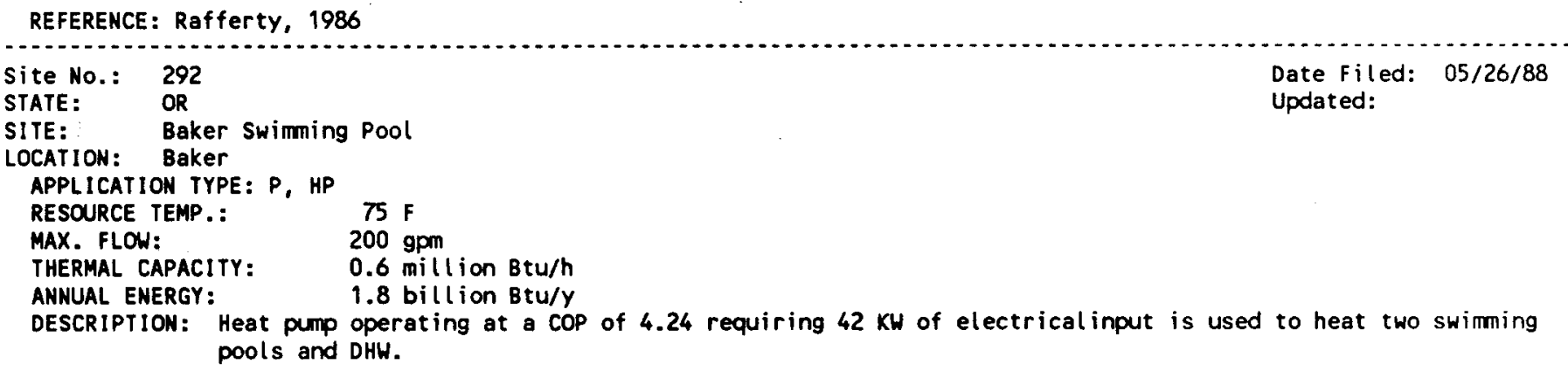

REFERENCE : MCGraw, 1984

Site No.: 293
STATE: $\quad$ OR
SITE:
LOCATION: Lakers Hot Spring
APPLICATION TYPE: S
RESOURCE TEMP.:
MAX. FLOH:
THERMAL CAPACITY:
ANNUAL ENERGY: $\quad 0.8 \mathrm{mill}$ ion Btu/h
DESCRIPTION: A motel is heated with geothermal fluid from one well, and another well drilled in 1923 produces a

REFERENCE: JUStUS, 1979

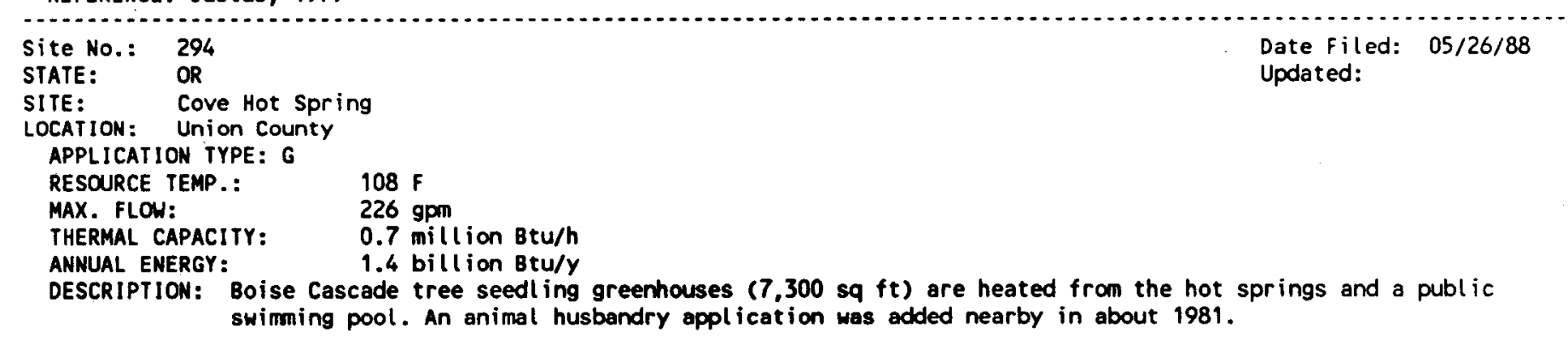

REFERENCE: Justus, 1979

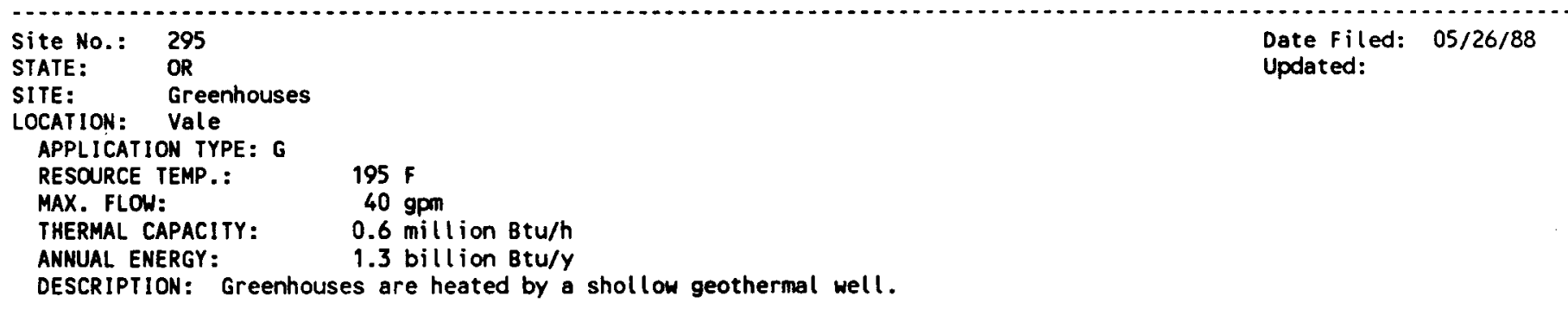


SITE: $\quad$ Medical Hot Springs

LOCATION: Union County APPLICATION TYPE: S, P RESOURCE TEMP.: $\quad 140 \mathrm{~F}$

MAX. FLOW: $\quad 100 \mathrm{gPm}$

THERMAL CAPACITY: $\quad 0.5 \mathrm{million}$ Btu/h

ANNUAL ENERGY: $\quad 1.1 \mathrm{bill}$ ion Btu/Y

DESCRIPTION: A resort and swimming pool are geothemally heated.

REFERENCE: Justus, 1979

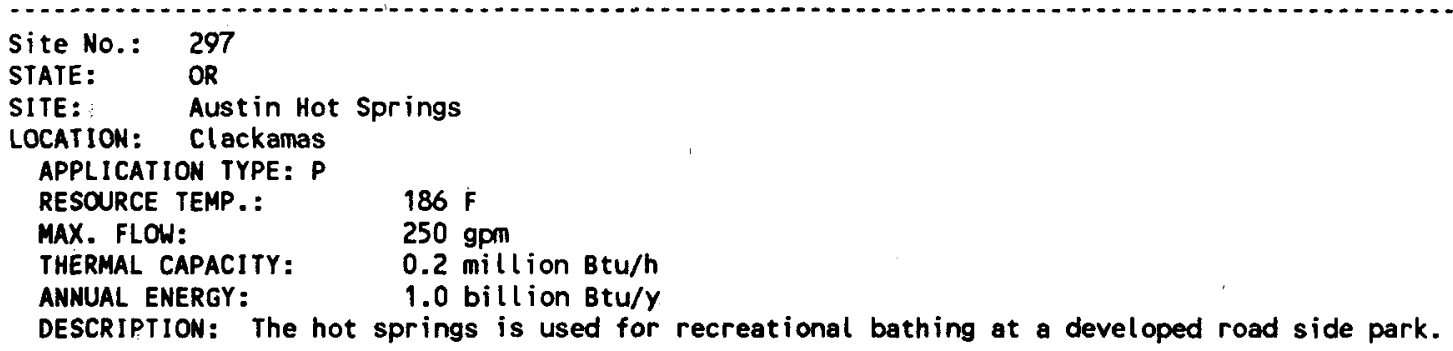

REFERENCE : Loam, 1980

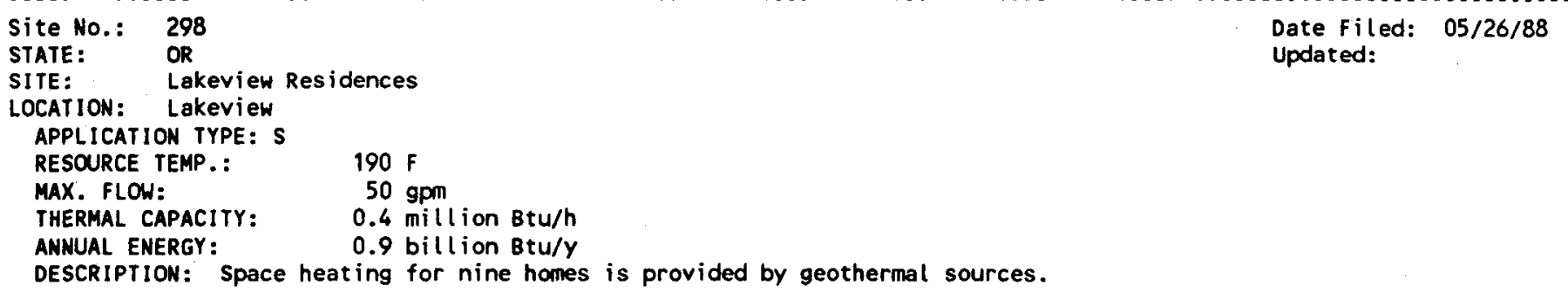

REFERENCE: Justus, 1979

Site No.: 299
STATE: $\quad$ OR
SITE: $\quad$ Vale slaughter House
LOCATION: Vale
APPLICATION TYPE: S
RESOURCE TEMP.: $\quad 150 \mathrm{~F}$
MAX. FLOW:
THERMAL CAPACITY: $\quad 0.3 \mathrm{gpm}$
ANNUAL ENERGY: $\quad 0.7$ billion Btu/h
DESCRIPTION: Thermal water is circulated in pipes buried in concrete floor for space heating and also used for
$\quad$ cleaning. Drilled new well - jet pump..
cleaning. Drilled new well - jet pump..

REFERENCE : Gannett, 1988

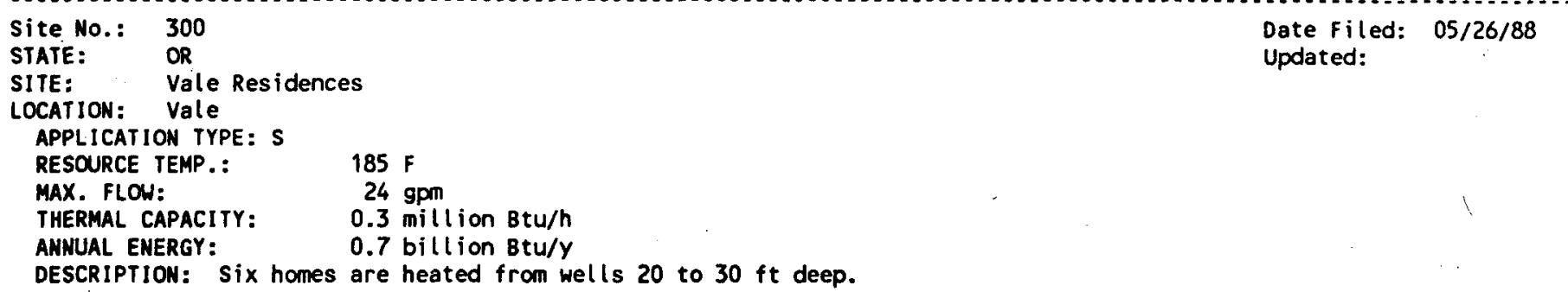


REFERENCE: Gannett, 1988

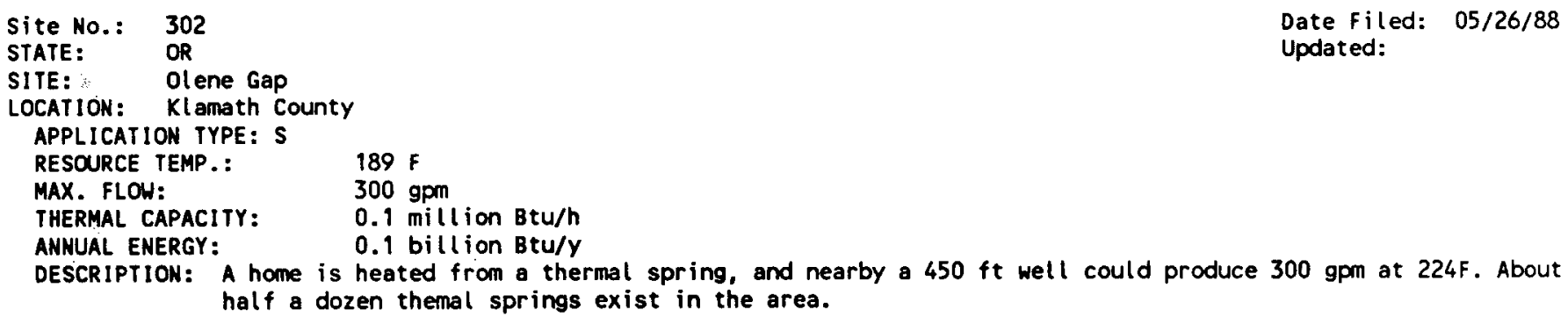

REFERENCE : Sammel, 1980

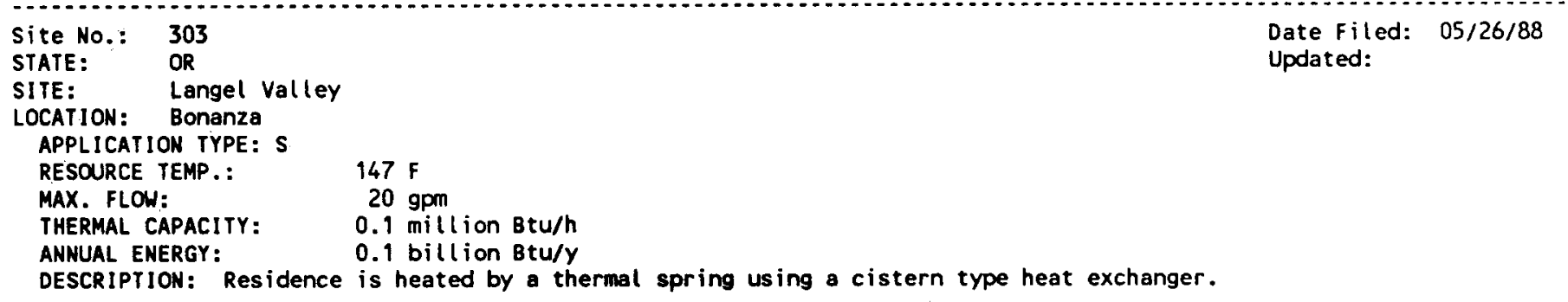

REFERENCE: Sammel, 1980

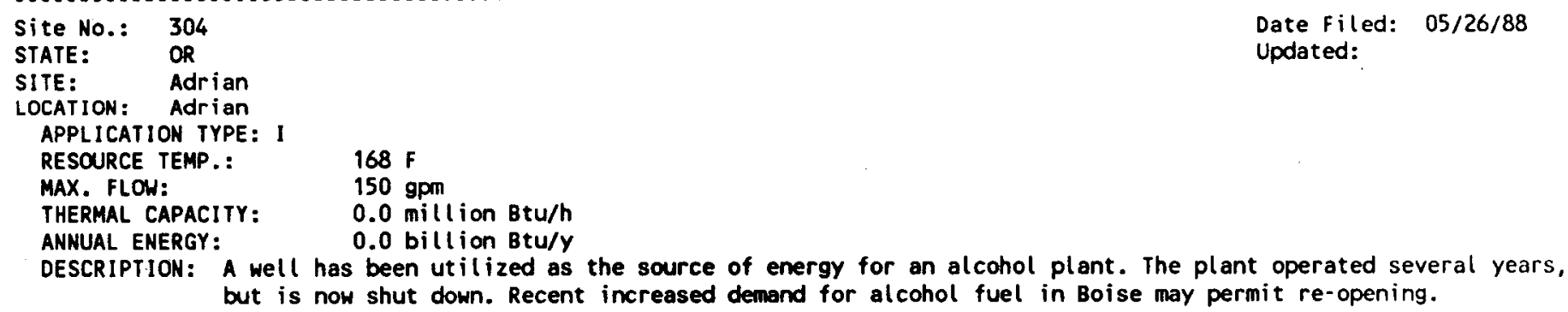

REFERENCE: Culver, 1988

Site No.: 305
STATE:
ORITE:
LOCATION: LUmer Lake Hot Springs
APPLICATION TYPE: S, A $109 \mathrm{~F}$
RESOURCE TEMP.: $\quad 20 \mathrm{gpm}$
MAX. FLOW:
THERMAL CAPACITY: $\quad$ million Btu/h
ANNUAL ENERGY:
DESCRIPTION: Space heating of two residences and a swimming pool at Summer Lake Hot Springs Resort. The Desert
Springs Trout Farm reportedly uses geothermal.
REFERENCE: Gannett, 1988


REFERENCE: Goodman, 1988

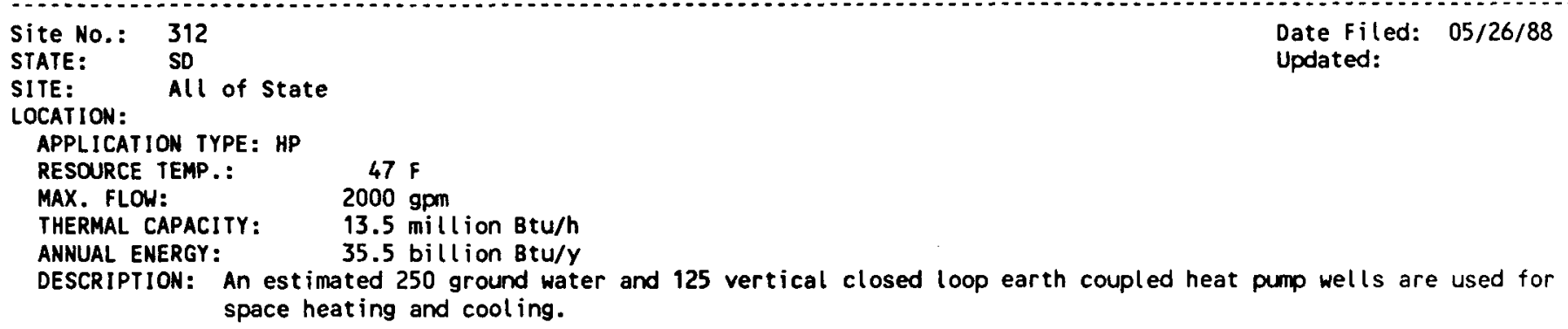

REFERENCE: Ellis, 1988

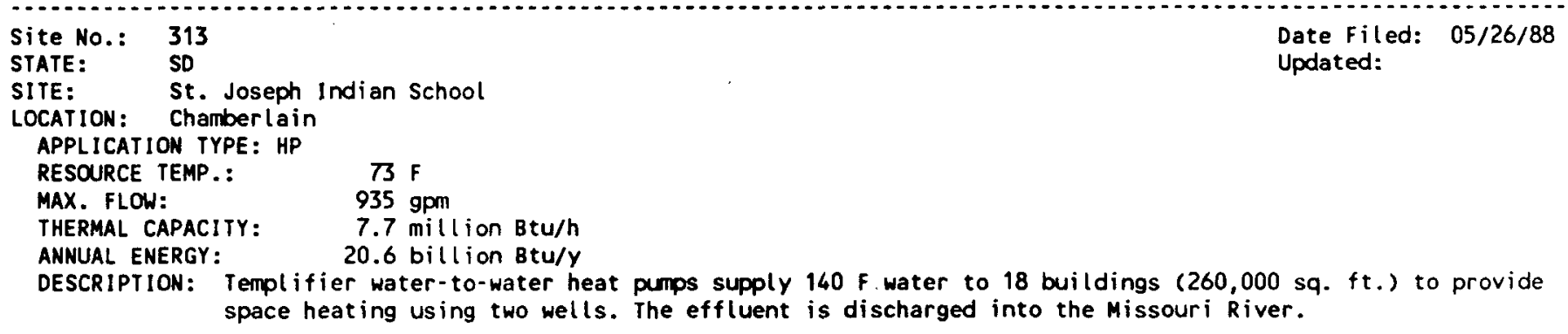

REFERENCE: Gonsorcik, 1988

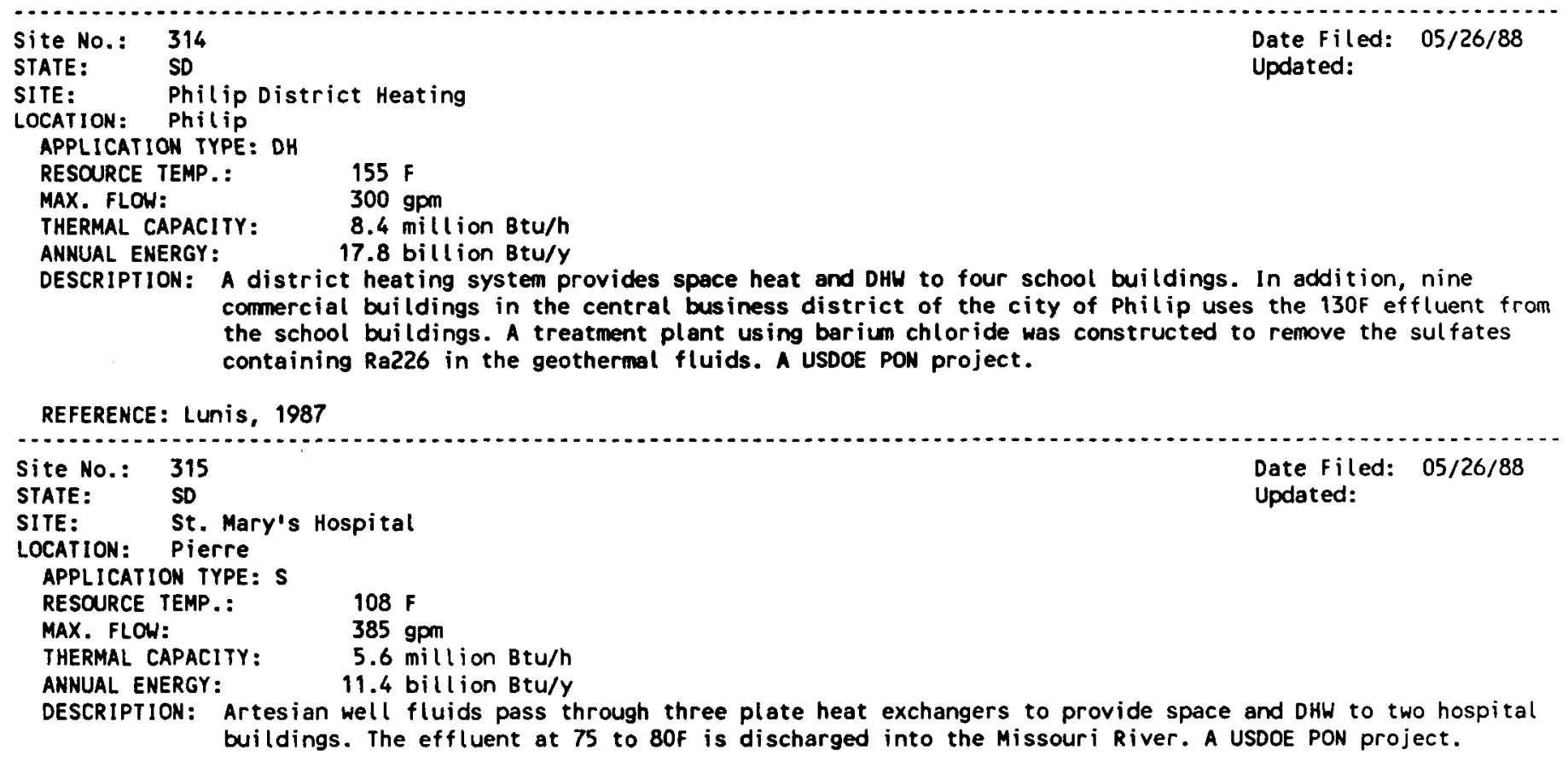


SITE: Lake Wagner Greenhouses

LOCATION: Philip

APPLICATION TYPE: $G$

RESOURCE TEMP.:

MAX. FLOW:

$154 \mathrm{~F}$

$250 \mathrm{gpm}$

THERMAL CAPACITY: $\quad 4.3 \mathrm{mill}$ ion $8 \mathrm{tu} / \mathrm{h}$

ANNUAL ENERGY: $\quad 9.3 \mathrm{bill}$ ion Btu/y

DESCRIPTION: An artesian well, about $1.5 \mathrm{mi} \mathrm{N}$ of Philip is used to provide space heat to about one acre of greenhouses. The well has a shut-in pressure of about $100 \mathrm{psig}$ and is used as the domestic water supply for Philip.

REFERENCE: Lienau, 1986

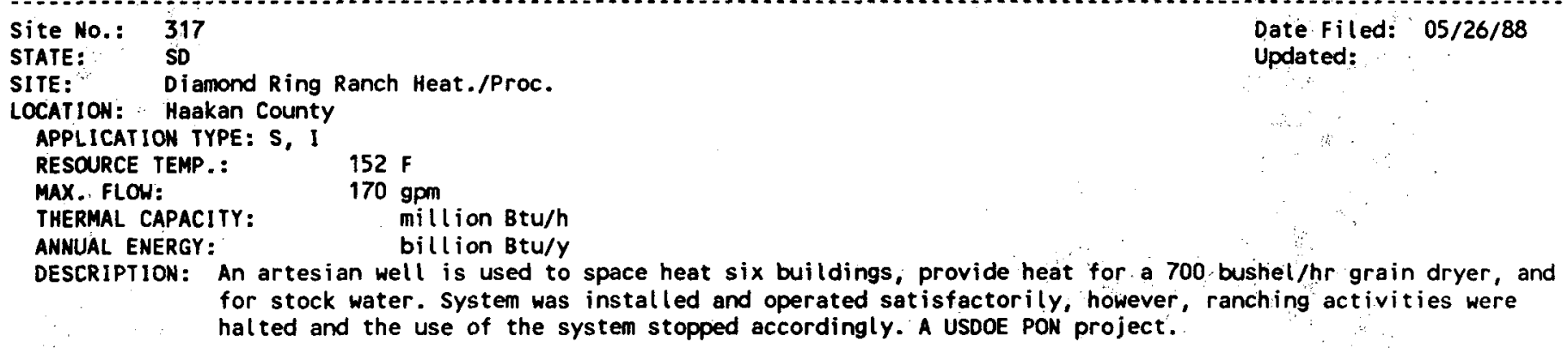

REFERENCE: Lunis, 1987

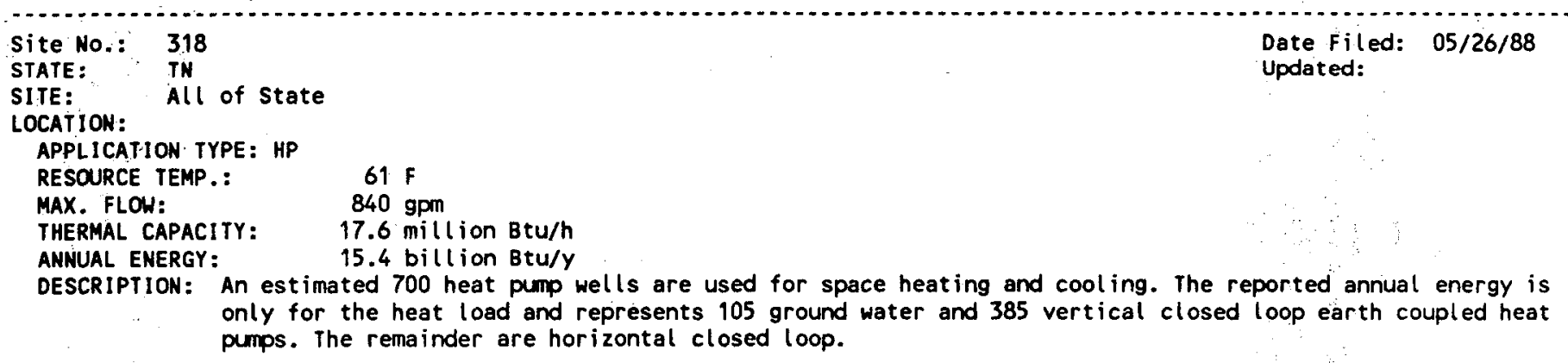

REFERENCE: Ellis, 1988

Site No.: 319

STATE: : TX

SITE: All of State

LOCATION: Corpus Cristy has large unit

APPLICATION TYPE: HP

RESOURCE TEMP.:

MAX. FLOW:

THERMAL CAPACITY:

ANNUAL ENERGY:

$3600 \mathrm{gpm}$

147.9 million Btu/h

129.6 billion Btu/y

An estimated 4050 heat pump wells are used for space heating and cooling. These well's represent 11 percent ground water and 89 percent vertical earth coupled.

REFERENCE: Ellis, 1988

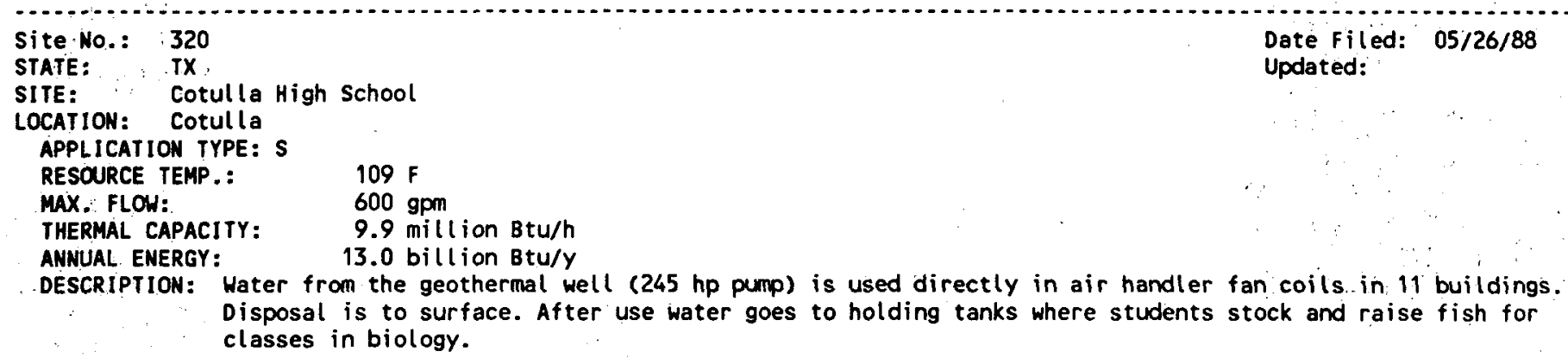


SITE: $\quad$ Stacy Park Pool

LOCATION: AUST In

APPLICATION TYPE: P

RESOURCE TEMP.: $\quad 96 \mathrm{~F}$

MAX. FLOW: $250 \mathrm{gpm}$

THERMAL CAPACITY: $\quad 0.9 \mathrm{mill}$ ion Btu/h

ANNUAL ENERGY: $\quad 5.5$ billion BtU/Y

DESCRIPTION: An outdoor pool approximately $100^{\prime} \times 40^{\prime}$ is headed between early fall and late spring by a $240^{\prime}$ pumped well. Pool is maintained at 82-84F most of the time; warmer some days.

REFERENCE: Aust in Park, 1988; C. Wicker

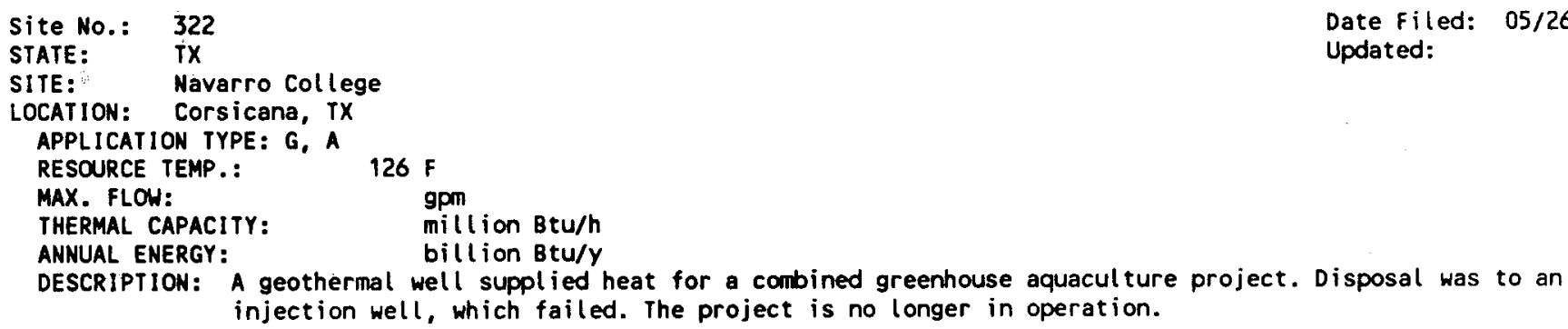

REFERENCE : Culver, 1988

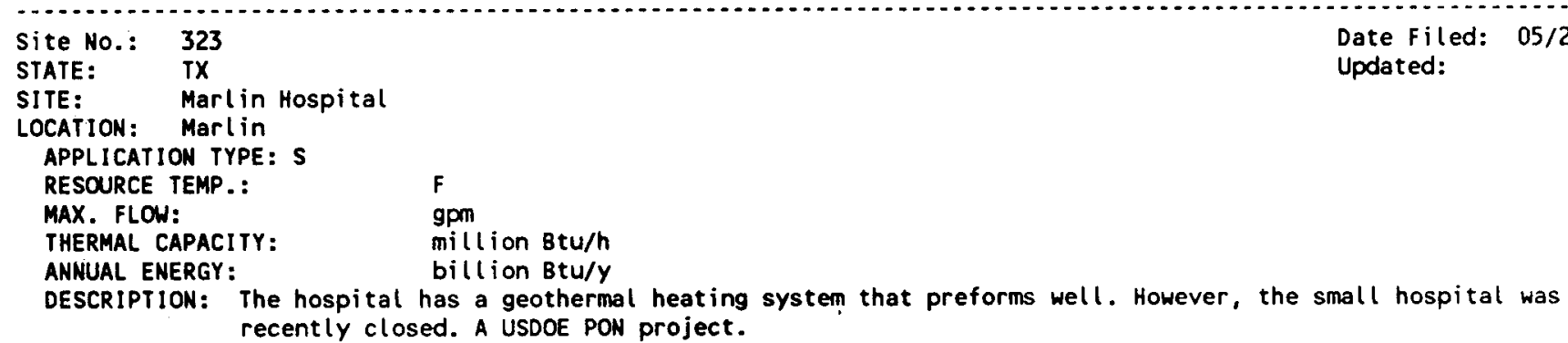

REFERENCE: BEG, 1988

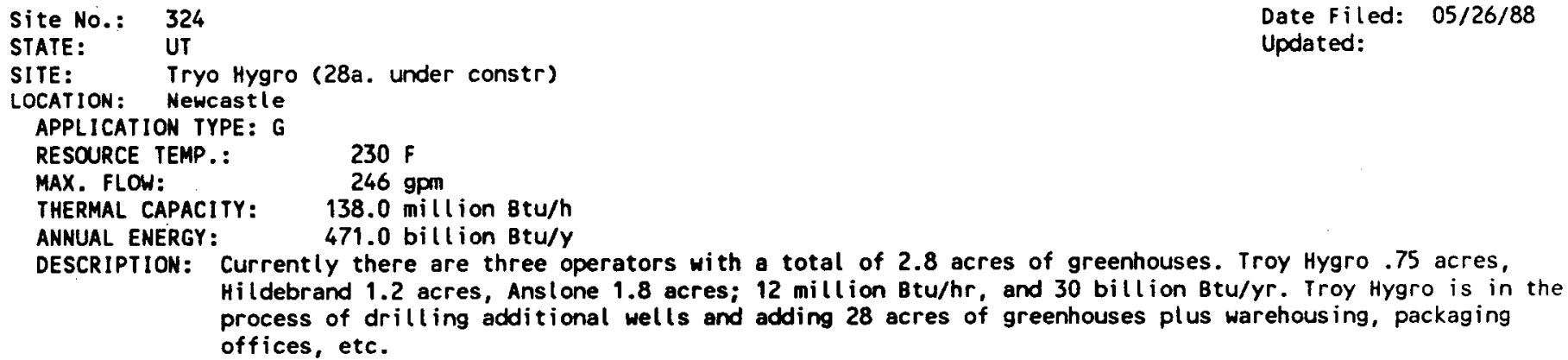

REFERENCE: Lienau, 1986; Troy Hygro, 1988.

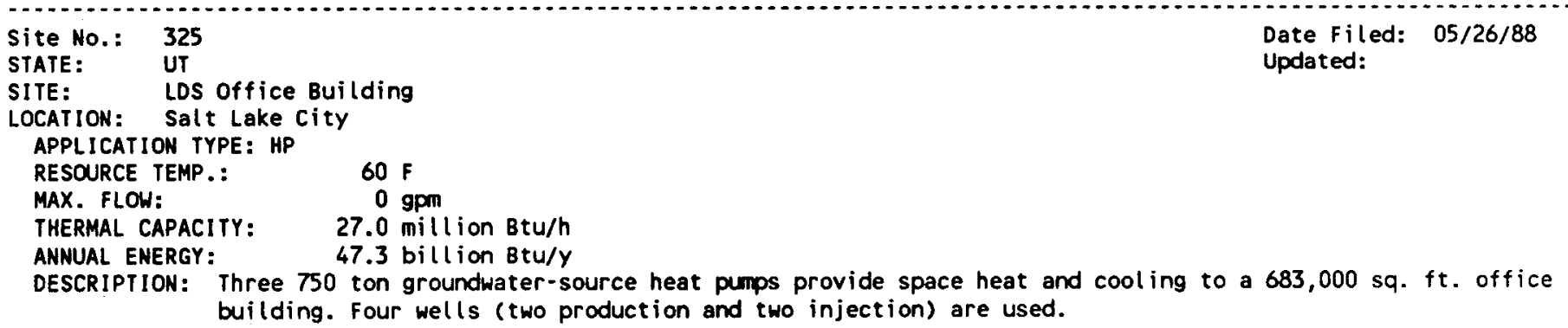


SITE: $\quad$ Saratoga Springs Resort Updated:

LOCATION: Lehi APPLICATION TYPE: S, P RESOURCE TEMP.: $120 \mathrm{~F}$ MAX. FLOW: $\quad 150 \mathrm{gpm}$ THERMAL CAPACITY: $\quad 2.0 \mathrm{mill}$ ion $8 \mathrm{tu} / \mathrm{h}$ ANNUAL ENERGY: $\quad 8.7$ billion 8 tu/y

DESCRIPTION: A lakeside recreation resort. Two outdoor swimming pools, one diving pool, two slide and spash pools. Hater for showers at the resort and space heating of 2 homes using radiant heat, 30 acre resort area.

REFERENCE : Eastmond, 1988

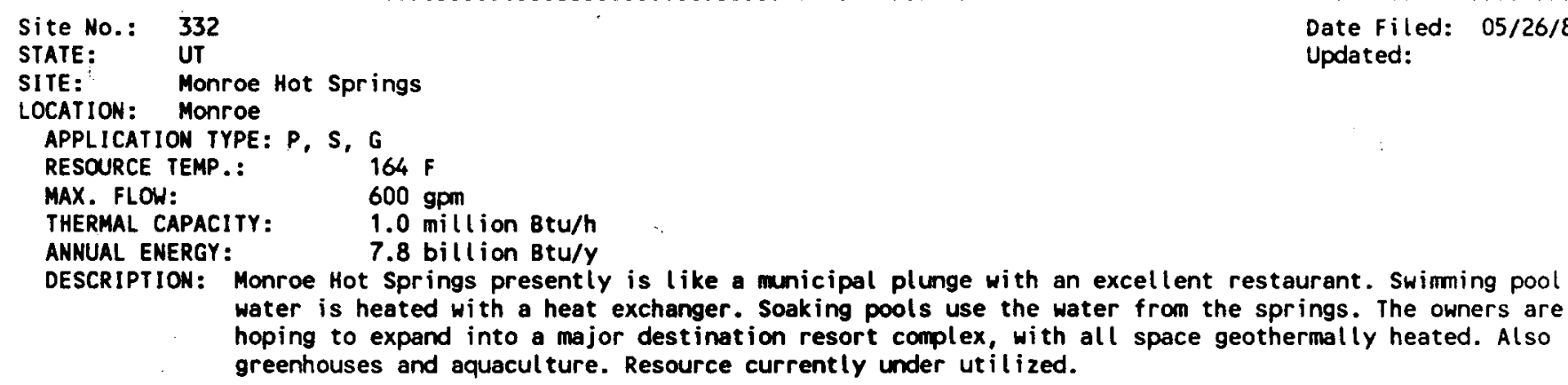
water is heated with a heat exchanger. Soaking pools use the water from the springs. The owners are hoping to expand into a major destination resort complex, with all space geothermally heated. Also greenhouses and aquacul ture. Resource currently under ut $i$ lized.

REFERENCE: Culver, 1988

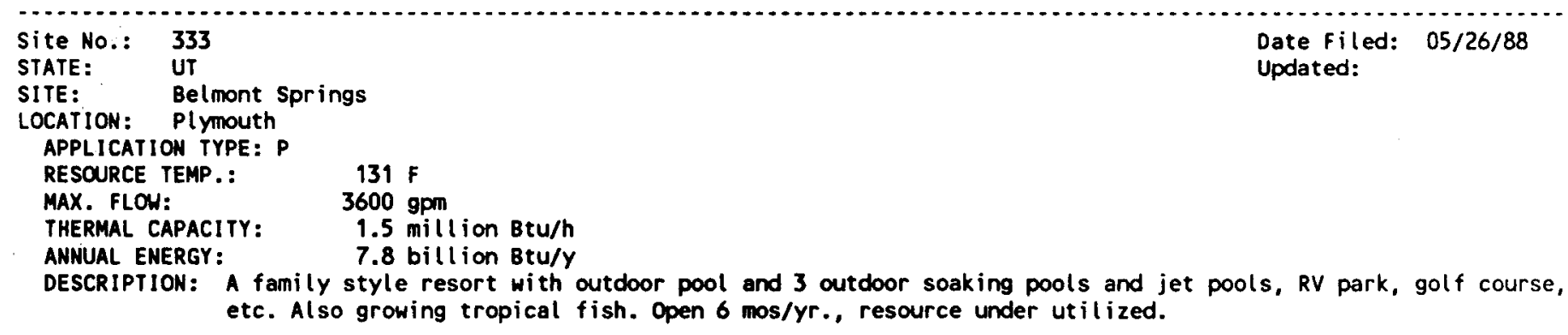

REFERENCE: Holmgren, 1988

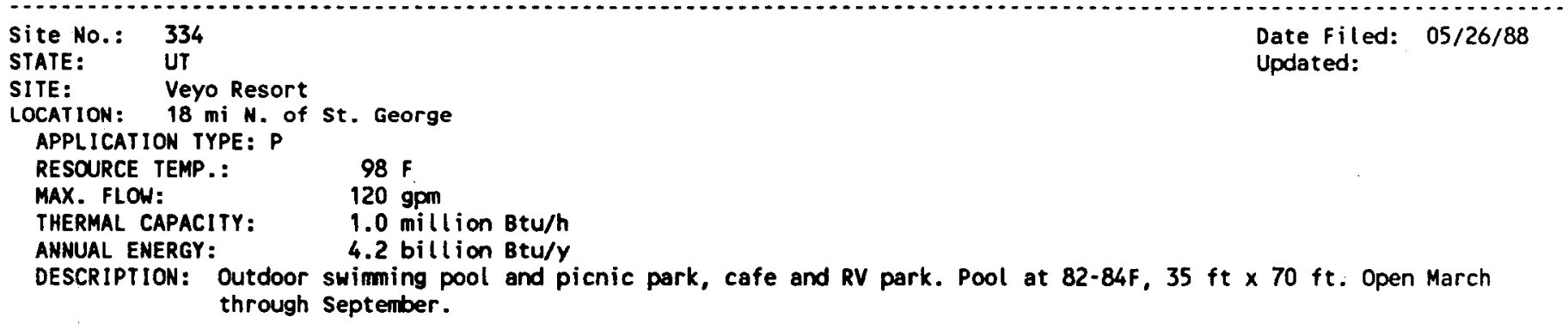

REFERENCE: Loam, 1980; Blacket, 1988

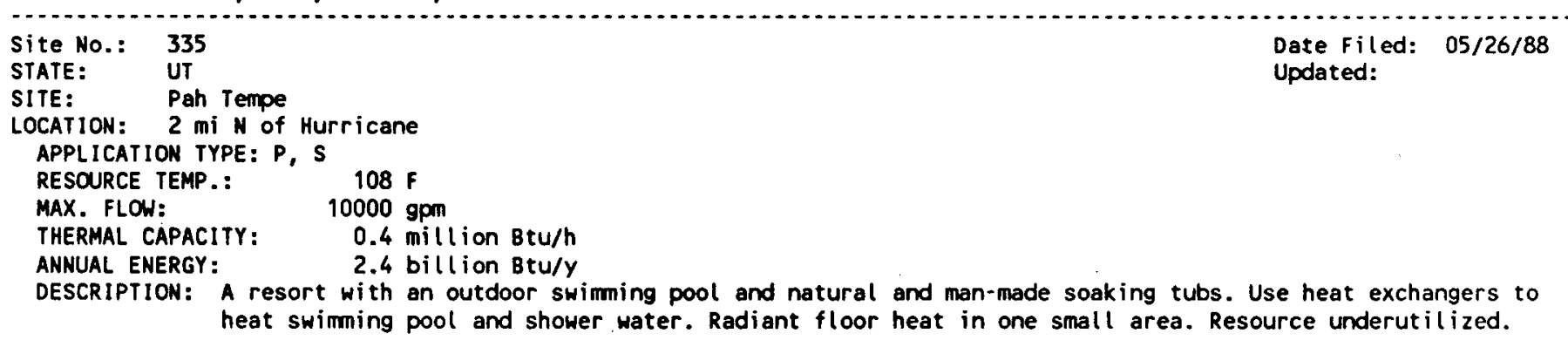




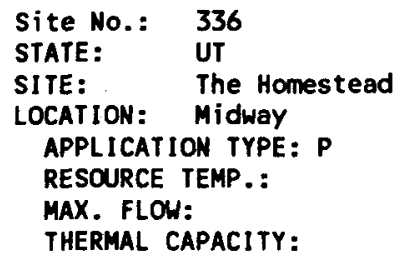
geothermal used only in 3 soaking pools. Investigating use for snow melting in sidewalks.

REFERENCE: Karrat, 1988

Site NO.: 337
STATE: UT
SITE: Mountain Spa Resort
LOCATION: Midway
APPLICATION TYPE: P
RESOURCE TEMP.:
MAX. FLOH: $115 \mathrm{~F}$
THERMAL CAPACITY: $1268 \mathrm{gpm}$
ANNUAL ENERGY: $\quad 0.5 \mathrm{million}$ Btu/h
DESCRIPTION: An old resort with an outdoor swiming pool, indoor swimming pool and soaking pools. Open only during
May - October. Resource grossly underut ilized.

REFERENCE : Kaysing, 1988; Blacket, 1988

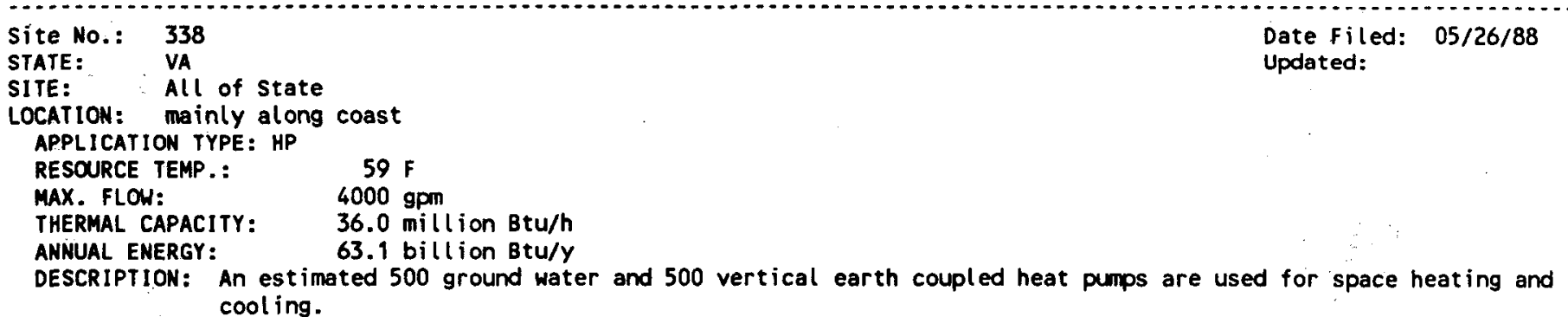

REFERENCE: Ellis, 1988

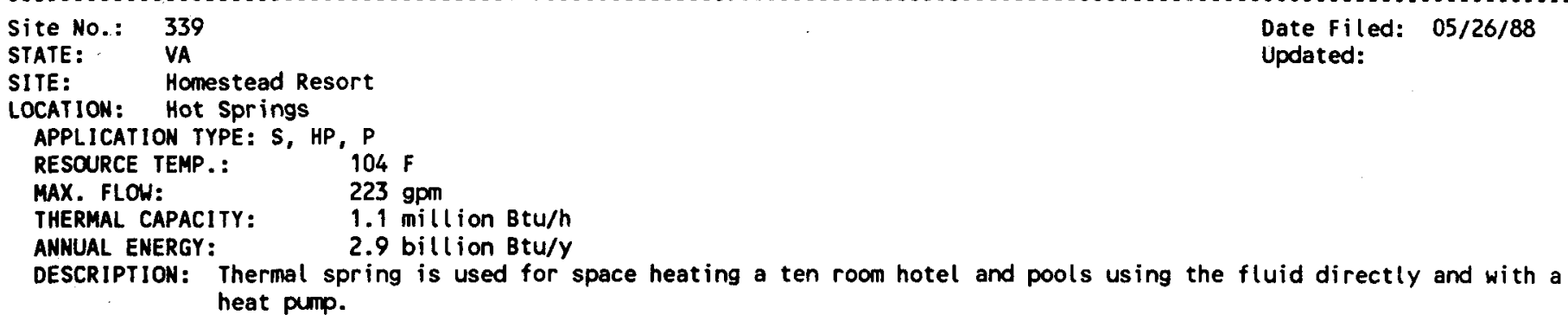

REFERENCE: Waldeck, 1988

Site NO.: 340
STATE: $\quad$ WA
SITE: Clark College
LOCATION: Vancouver
APPLICATION TYPE: HP
RESOURCE TEMP.:
MAX. FLOW: $55 \mathrm{~F}$
THERMAL CAPACITY: $\quad 650 \mathrm{gpm}$
ANNUAL ENERGY: $\quad 13.8 \mathrm{million}$ billion Btu/h
DESCRIPTION: Space heating and cooling of 15 buildings is accomplished by a water-to-water heat pump, utilizing a


SITE: $\quad$ Grant County Courthouse

LOCATION: Ephrata

APPLICATION TYPE: HP

RESOURCE TEMP.: $\quad 84 \mathrm{~F}$

MAX. FLOW:

THERMAL CAPACITY:

ANNUAL ENERGY:

$600 \mathrm{gpm}$

3.7 million Btu/h

B. 4 billion Btu/y

DESCRIPTION: A heat pump operates at a COP of 5.77 and requires $188 \mathrm{KH}$ electrical input for space heating and cooling. Project was developed with a HUD grant.

REFERENCE: MCGraw, 1985

Site No.: 342
STATE: $\quad$ WA
SITE: : Yakima County Jail
LOCATION: Yakima
APPLICATION TYPE: HP
RESOURCE TEMP.:
MAX. FLOH:
THERMAL CAPACITY: $\quad 300 \mathrm{fpm}$
ANMUAL ENERGY: $\quad 3.6 \mathrm{million}$ Btu/h
DESCRIPTION: Heat pump system is used for space heating and cooling the 120,000 sq ft facility using an $800 \mathrm{ft}$
$\quad$ production well and $600 \mathrm{ft}$ injection well as the energy source.

REFERENCE: DeLUCa, 1988

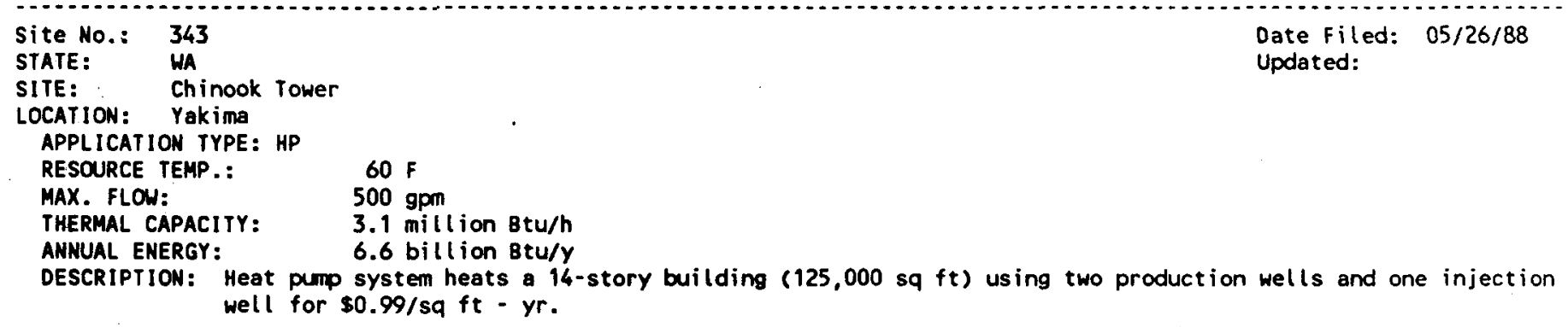

REFERENCE: DeLuCa, 1988

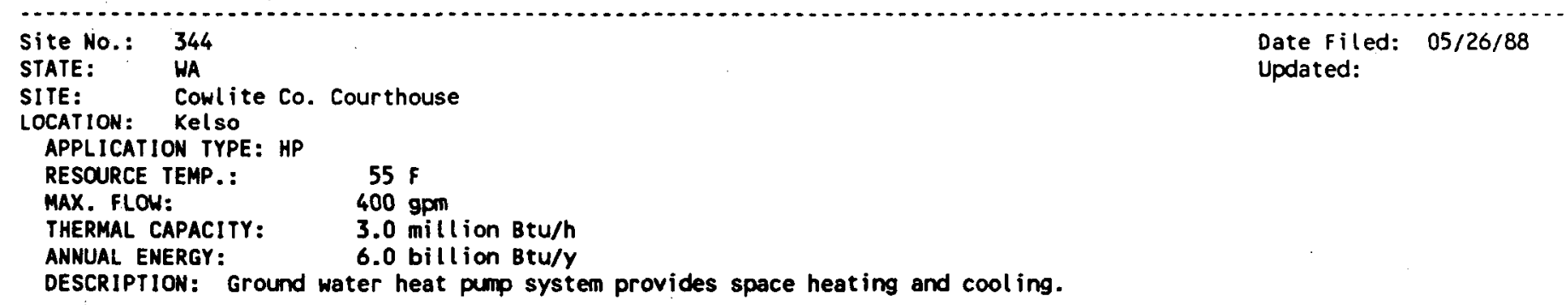

REFERENCE: Bloomquist, 1988

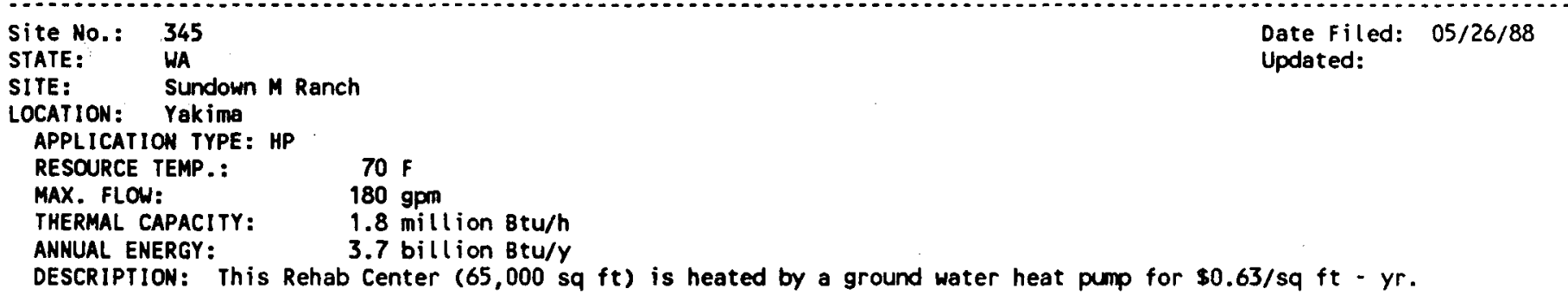


SITE: Sol Duc Hot Springs

LOCATION: Clallam County

APPLICATION TYPE: P

RESOURCE TEMP.: $128 \mathrm{~F}$

MAX. FLOW: $\quad 50 \mathrm{gpm}$

THERMAL CAPACITY: $\quad 0.5 \mathrm{mill}$ ion Btu/h

ANNUAL ENERGY: $\quad 1.2$ billion Btu/Y

DESCRIPTION: A large swimming pool and mineral pool are heated with thermal spring water and is being operated by a concession aire for the Olympic National Park.

REFERENCE: Bloomquist, 1979

Site NO.: 347
STATE:
SITE: WA
LOCATION: Adams Co. Fire Station
APPLICATION TYPE: HP
RESOURCE TEMP.:
MAX. FLOW:
THERMAL CAPACITY:
ANNUAL ENERGY:
DESCRIPTION: A ground water heat pump syst

Date filed: $05 / 26 / 88$ Updated:

DESCRIPTION: A ground water heat pump syste years, for a fire station.

REFERENCE : Rafferty, 1982

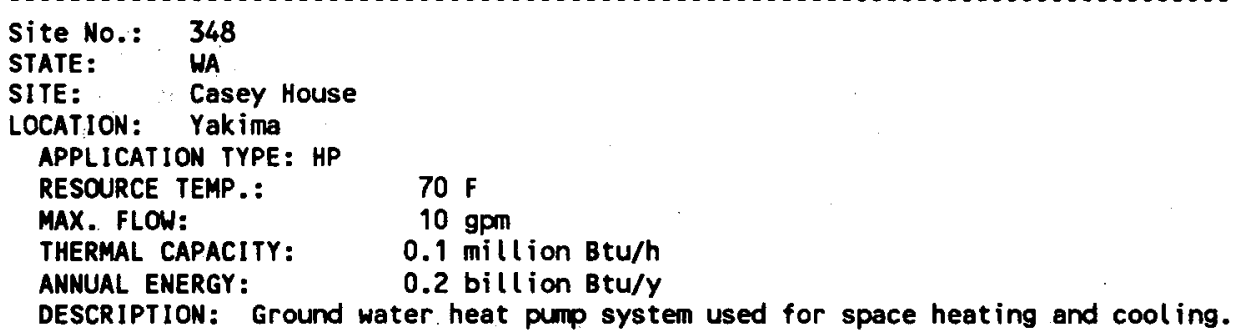

REFERENCE: DeLUCa, 1988

Site No.: $\quad 349$
STATE:
SITE: HA
LOCATION: Yarm Credit Services Bldg.

REFERENCE: DeLUCA, 1988

Site No.: 350
STATE: $\quad$ WA
SITE: $\quad$ Skove Bldg.
LOCATION: Yakima
APPLICATION TYPE: HP
RESOURCE TEMP.:
MAX. FLOW:
THERMAL CAPACITY:
ANNUAL ENERGY:
DESCRIPTION: Ground water heat pump system used for space heating and cooling.


LOCATION: Yakima APPLICATION TYPE: HP RESOURCE TEMP.:

MAX. FLOW:

THERMAL CAPACITY:

$56 \mathrm{~F}$ gpm

million Btu/h billion Btu/y

ANNUAL ENERGY:

heat pump system uses a geothermal well for space heating and cooling.

REFERENCE: DeLUCa, 1988

Site No.: 352
STATE: $\quad$ WA
SITE: $\quad$ Nazarene Church
LOCATION: Yakima
APPLICATION TYPE: HP
RESOURCE. TEMP.:
MAX. FLOW:
THERMAL CAPACITY:
ANNUAL ENERGY:
DESCRIPTION: Ground water heat pump system, used for space heating and cooling.

REFERENCE: DeLLCa, 1988

$\begin{array}{lll}\text { Site No.: } & 353 & \text { Date filed: } \\ \text { STATE: } & \text { WA } & 05 / 26 / 88 \\ \text { SITE: } & \text { Grant CO. PUD } & \text { Updated: }\end{array}$

SITE: Grant CO. PUD

SITEAION: Ephrata APPLICATION TYPE: HP RESOURCE TEMP.:

MAX. FLOW:

THERMAL CAPACITY:

ANNUAL ENERGY:

$80 \mathrm{~F}$ gpm

million Btu/h

billion Btu/y

DESCRIPIION: Ground water heat pump provides space heating.

REFERENCE: Bloomquist, 1988

$\begin{array}{ll}\text { Site NO.: } & 354 \\ \text { STATE: } & \text { HA } \\ \text { SITE: } \quad \text { Red Cross Bldg. } \\ \text { LOCATION: Yakima } \\ \text { APPLICATION TYPE: HP } \\ \text { RESOURCE TEMP.: } \\ \text { MAX. FLOW: } & \text { Fpm } \\ \text { THERMAL CAPACITY: } & \text { million Btu/h } \\ \text { ANNUAL ENERGY: } & \text { billion Btu/y }\end{array}$

DESCRIPTION: Ground water heat pump system used for space heating and cooling.

REFERENCE: Delucs, 1988

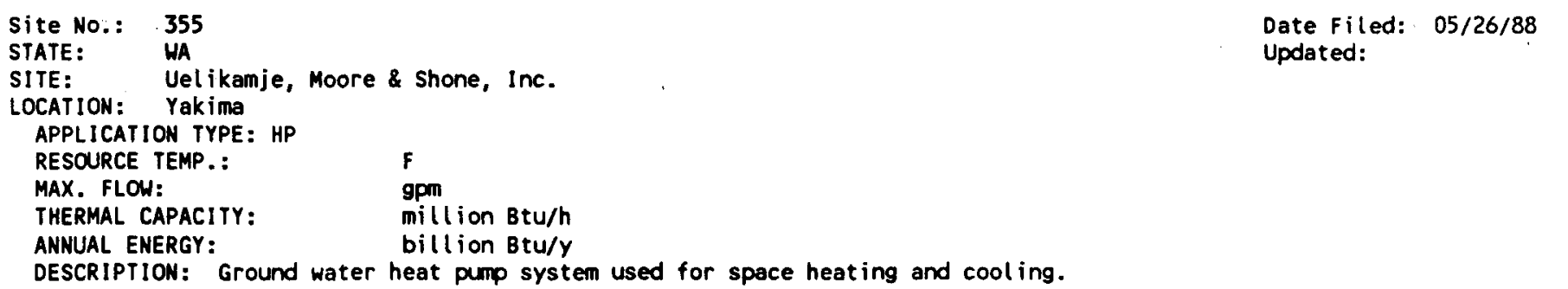


LOCATION: Tacoma

APPLICATION TYPE: HP

RESOURCE TEMP.:

MAX. FLOW:

THERMAL CAPACITY:

\section{$F$}

gpm

million Btu/h

billion Btu/Y

DESCRIPIION: Ground water heat pump system uses a geothermal well.

REFERENCE : Bloomquist, 1988

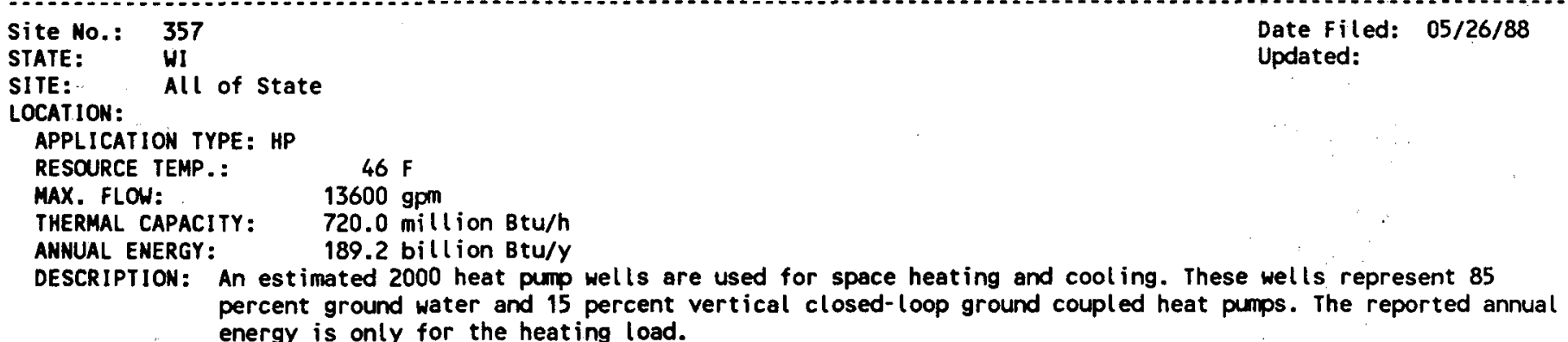
energy is only for the heating load.

REFERENCE: Ellis, 1988

$\begin{array}{ll}\text { Site NO:: } & 358 \\ \text { STATE: } & \text { HY } \\ \text { SITE: } & \text { Secondary Oil Recovery } \\ \text { LOCATION: Near Medwest (Natrona CO.) } \\ \text { APPLICATION TYPE: I } \\ \text { RESOURCE TEMP.: } & 200 \mathrm{~F} \\ \text { MAX. FLOW: } & 8300 \mathrm{gpm} \\ \text { THERMAL CAPACITY: } & 498.3 \mathrm{mill} \text { ion Btu/h } \\ \text { ANNUAL ENERGY: } & 3492.1 \mathrm{bill} \text { ion Btu/y }\end{array}$

Date Filed: $05 / 26 / 88$

Updated: $08 / 19 / 88$

ENERGY: 3492.1 biltion Btu/y and is injected for secondary oil recovery from oil well. This heat is extremely useful in increasing the recovery of up to an additional third of the original oil.

REFERENCE : Reed, 1988

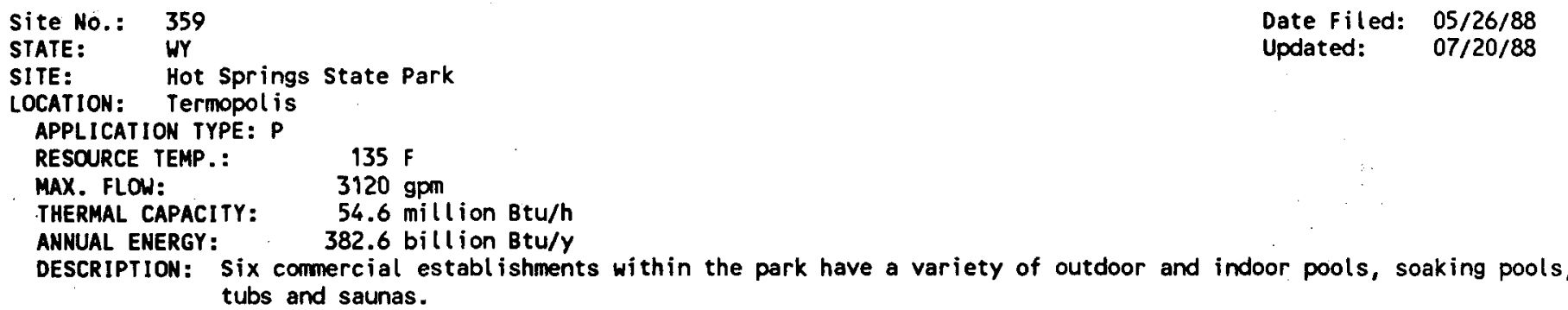

REFERENCE : Loam, 1980

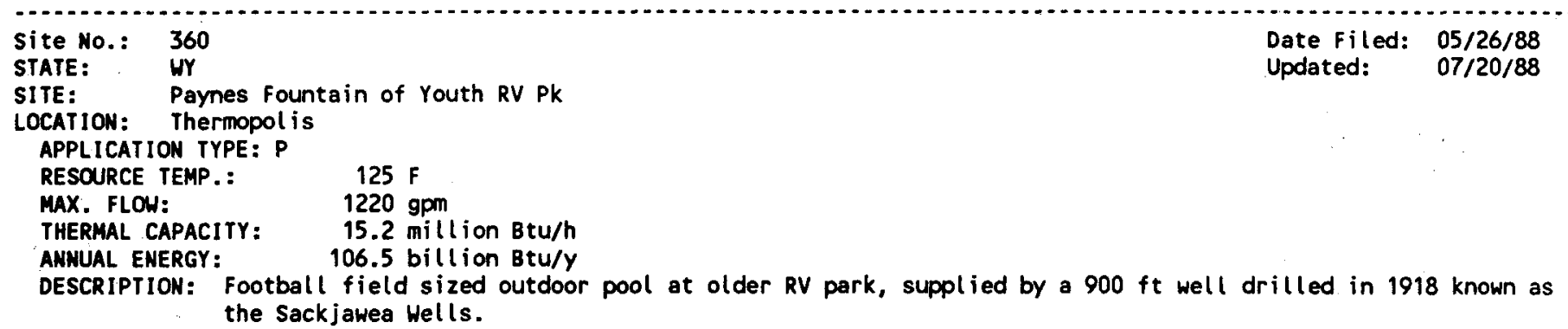


Site NO.: 361
STATE: $\quad$ WY
SITE: $\quad$ The Saratoga Inn
LOCATION: Saratoga
APPLICATION TYPE: P
RESOURCE TEMP.: $\quad 114 \mathrm{~F}$
MAX. FLOW:
THERMAL CAPACITY: $\quad 450 \mathrm{gPm}$
ANNUAL ENERGY: $\quad 3.5 \mathrm{million} \mathrm{Btu/h}$
OESCRIPTION: A golf and ternis resort with a large outdoor pool.

REFERENCE : Kaysing, 1985; Loam, 1980
Date Filed: $05 / 26 / 88$ Updated:

Site No.: 362

STATE: WY HY

SITE: $\quad$ Huckelberry Hot Springs

LOCATION: Grand Teton Nat'l Park

APPLICATION TYPE: P, I

RESOURCE TEMP.: $140 \mathrm{~F}$

MAX. FLOW: $\quad 300 \mathrm{gpm}$

THERMAL CAPACITY: $\quad 4.2 \mathrm{mill}$ ion BtU/h

ANNUAL ENERGY: $\quad 29.4$ billion Btu/Y

DESCRIPTION: Commercial resort on leased land in the park. A large outdoor swimming pool, store, RV park, etc. Geothermal water is also used in the laundry.

REFERENCE : Kaysing, 1985

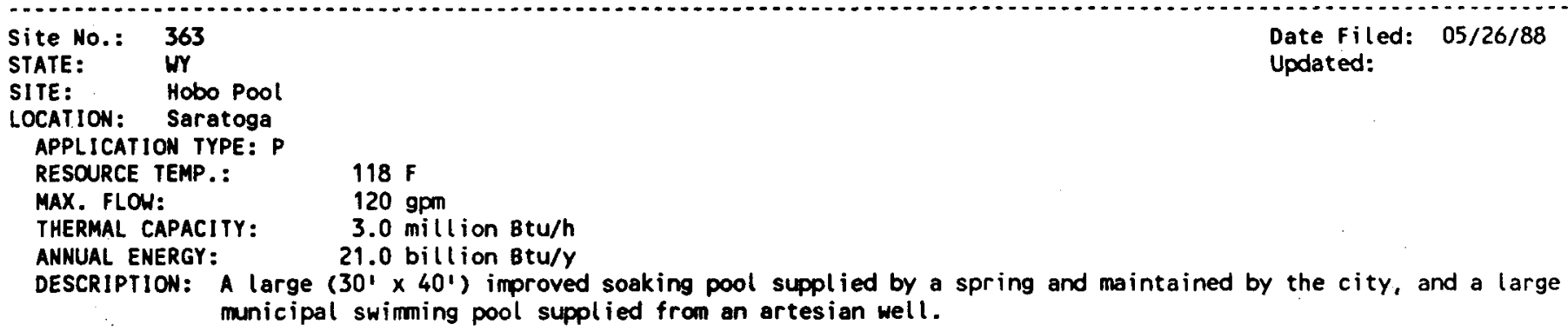

REFERENCE：James, 1979

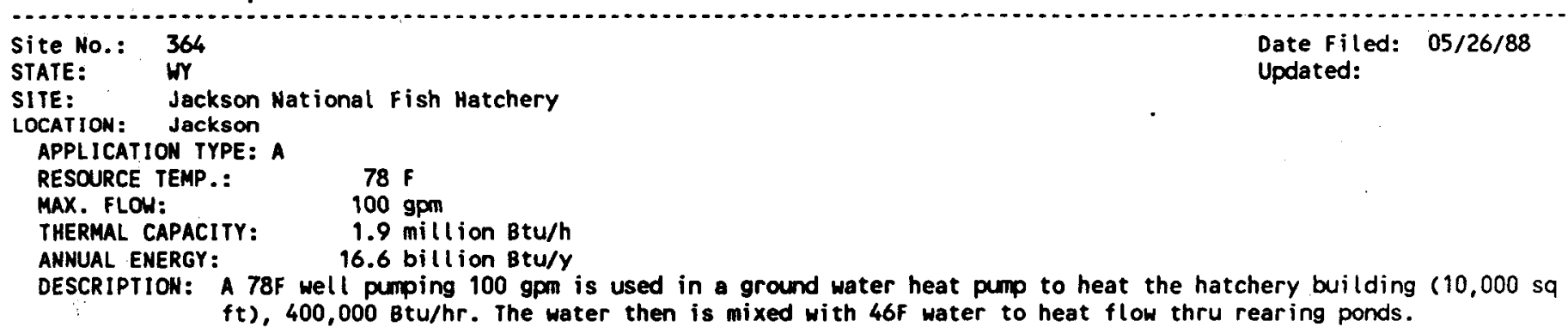

REFERENCE :

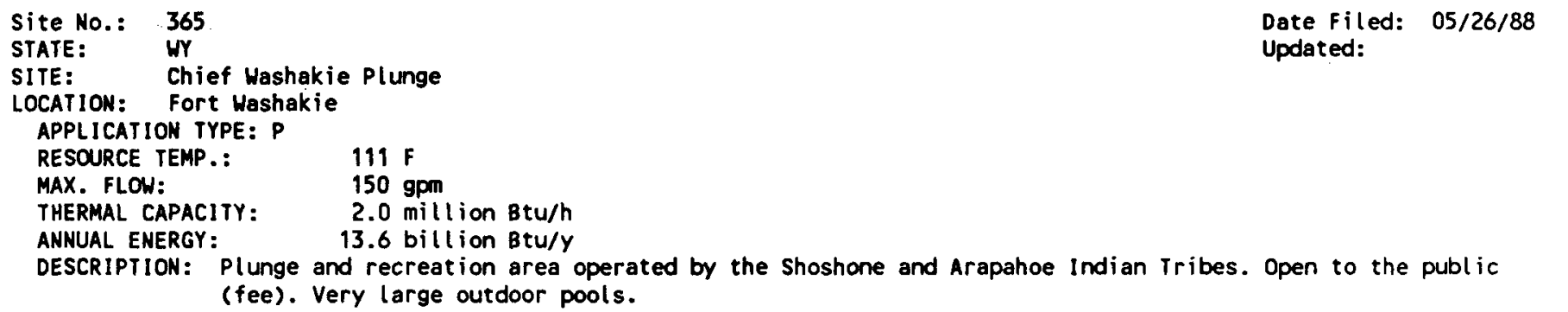


SITE: Cody Athletic Club

LOCATION: COOY

APPLICATION TYPE: P

RESOURCE TEMP.: $102 \mathrm{~F}$

MAX. FLOW: $250 \mathrm{gpm}$

THERMAL CAPACITY: $\quad 1.5$ million Btu/h

ANNUAL ENERGY: $\quad 10.5$ billion BtU/Y

DESCRIPTION: Fully equipped health club and motel with large outdoor pool.

REFERENCE: Heasler, 1988

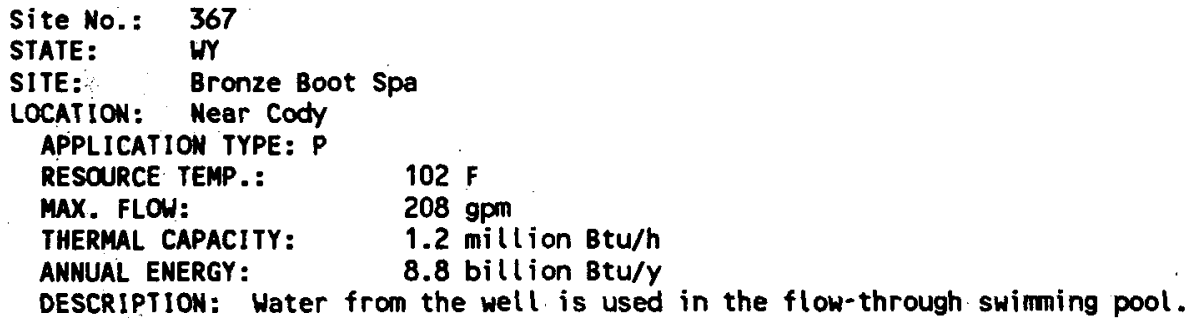

REFERENCE: : Breckenridge, 1988

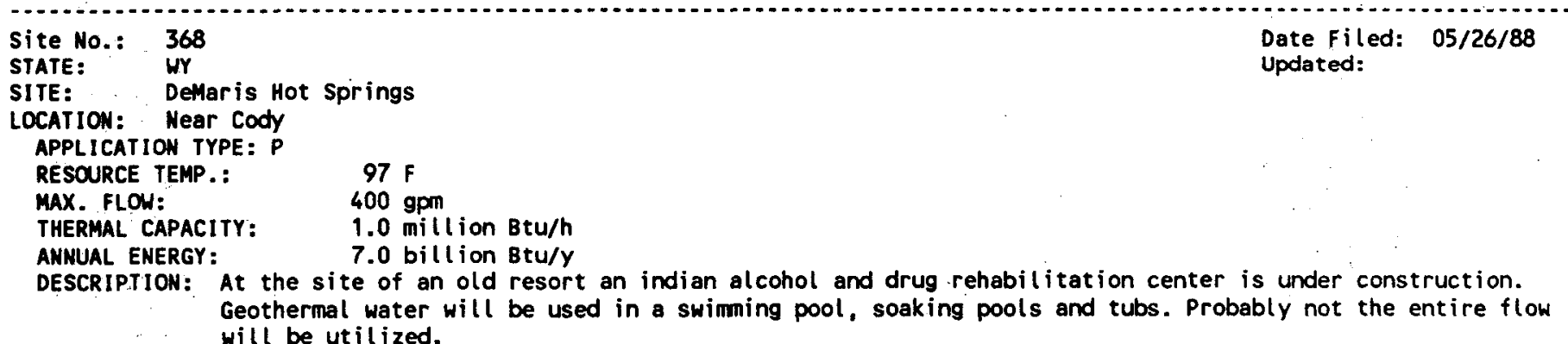

REFERENCE : Easton, 1988

Site No.: 369

STATE: WY

SITE: Astoria Mineral Hot Springs

LOCATION: $17 \mathrm{mi}$. SO. Of Jackson

APPLICATION TYPE: P

RESOURCE TEMP.: $\quad 104 \mathrm{~F}$

MAX. FLOW: $\quad 100 \mathrm{gpm}$

THERMAL CAPACITY: $\quad 1.0 \mathrm{mill}$ ion Btu/h

ANMUAL ENERGY: $\quad 6.6$ billion Btu/Y

DESCRIPTION: A modern resort with outdoor pool, store, RV park, etc.
Date Filed: $05 / 26 / 88$ Updated: 


\section{REFERENCE: James, 1979}

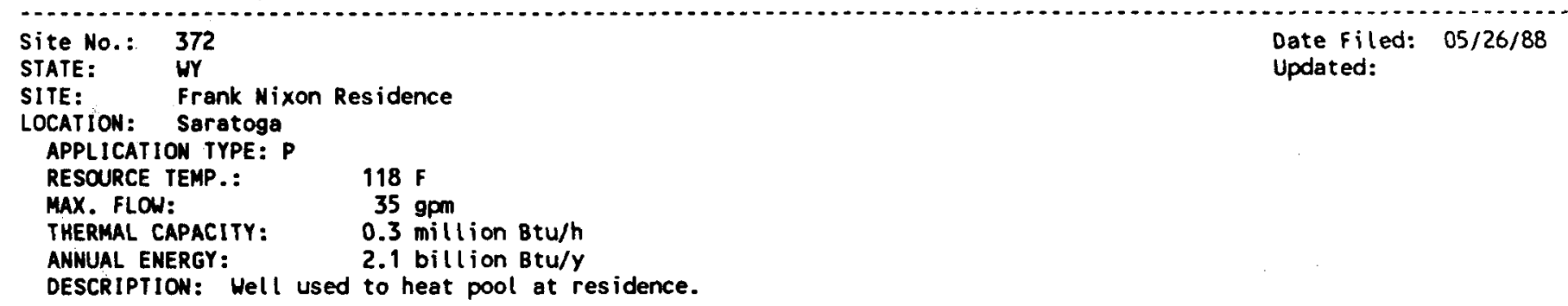

REFERENCE: James, 1979

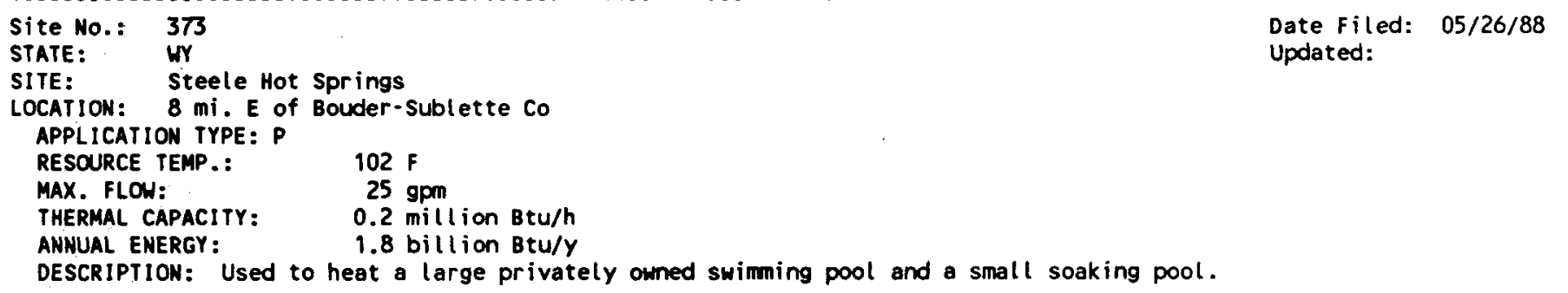

REFERENCE : Breckenridge, 1988

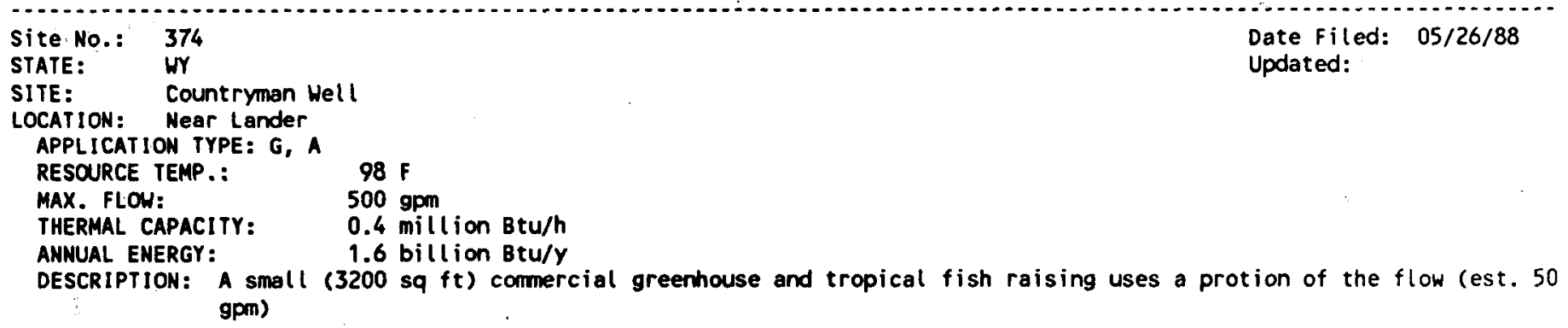

REFERENCE : Breckenridge, 1988

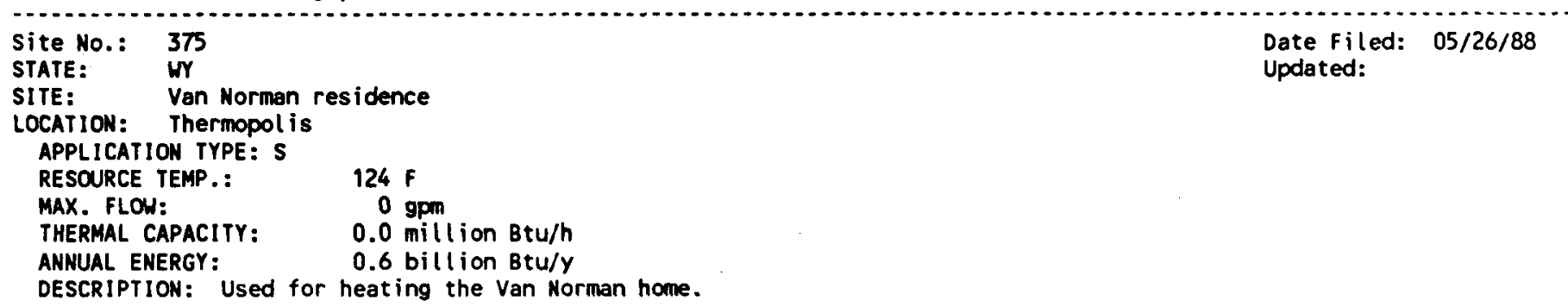


SITE: $\quad 1-80$ 16th St. off ramp

LOCATION: Cheyenne

APPLICATION TYPE: I

RESOURCE TEMP.:

MAX. FLOW:

$0 \mathrm{gpm}$

THERMAL CAPACITY:

ANNUAL ENERGY:

0.9 million Btu/h

billion Btu/y

OESCRIPTION: Three hundred forty 100-ft deep wells with down-hole heat exchangers circulate ammonia in a closed-loop highway de-icing system.

REFERENCE : Nydahl, 1988

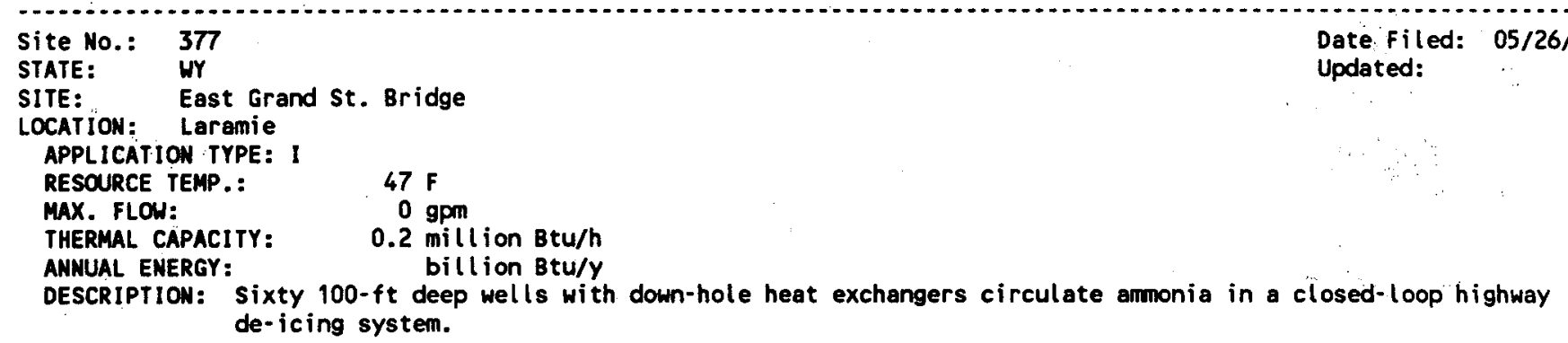

